Prepared in cooperation with the Bureau of Land Management, City of Gunnison, Colorado River Water Conservation District, Crested Butte South Metropolitan District, Gunnison County, Hinsdale County, Mount Crested Butte Water and Sanitation District, National Park Service, Town of Crested Butte, U.S. Forest Service, Upper Gunnison River Water Conservancy District, and Western State College

\title{
Comparison of 2008-2009 Water Years and Historical Water-Quality Data, Upper Gunnison River Basin, Colorado
}

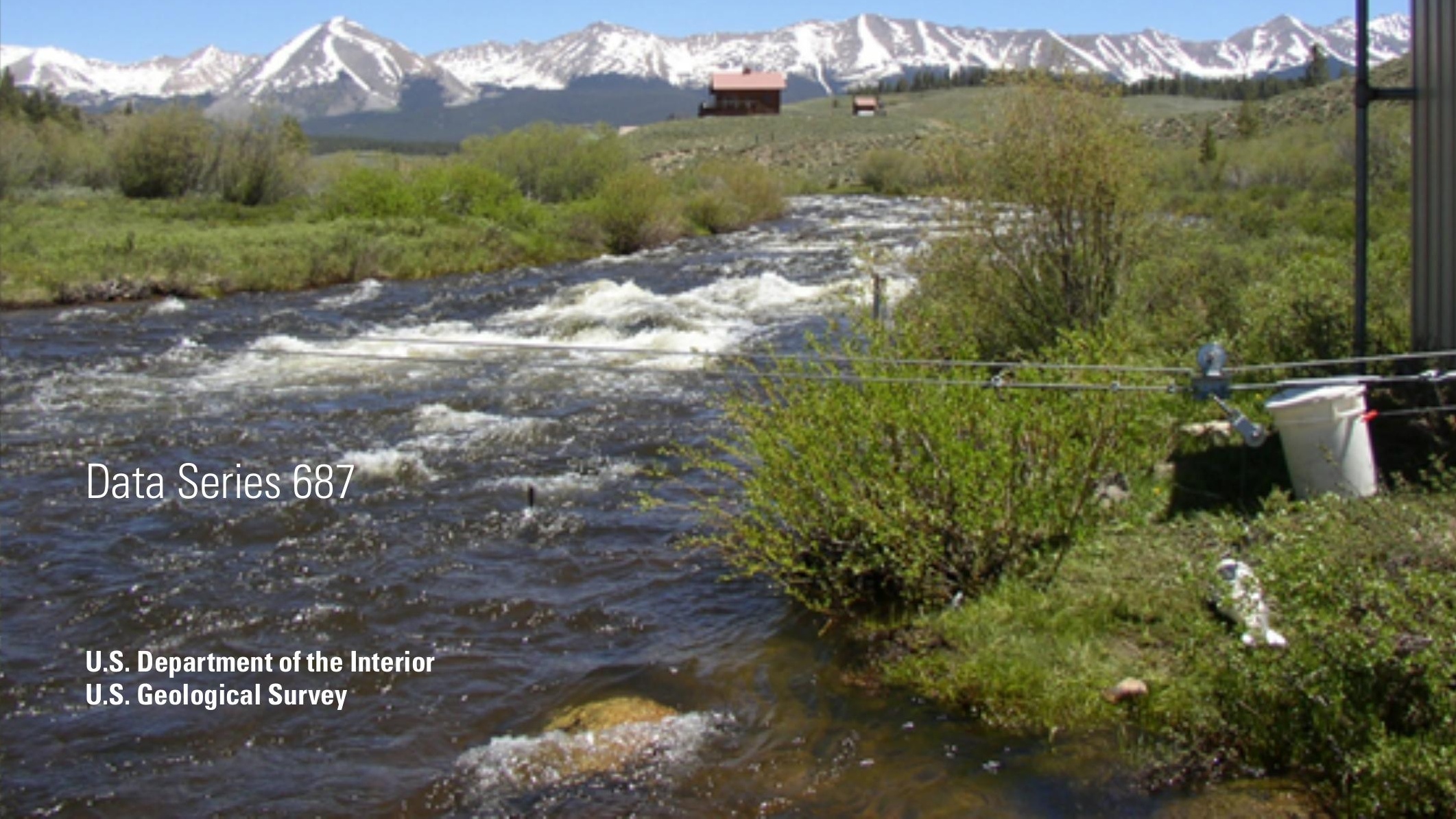


Cover: Looking upstream from the Taylor River at Taylor Park, Colorado, streamgage approximately 400 feet upstream from the confluence with Taylor Park Reservoir, June 2011 (Photograph taken by John Sottilare, U.S. Geological Survey). 


\section{Comparison of 2008-2009 Water Years and Historical Water-Quality Data, Upper Gunnison River Basin, Colorado}

By Patricia A. Solberg, Bryan Moore, and Ty D. Blacklock

Prepared in cooperation with the

Bureau of Land Management

City of Gunnison

Colorado River Water Conservation District

Crested Butte South Metropolitan District

Gunnison County, Hinsdale County

Mount Crested Butte Water and Sanitation District

National Park Service

Town of Crested Butte

U.S. Forest Service

Upper Gunnison River Water Conservancy District

Western State College

Data Series 687 


\title{
U.S. Department of the Interior \\ KEN SALAZAR, Secretary \\ U.S. Geological Survey \\ Marcia K. McNutt, Director
}

\author{
U.S. Geological Survey, Reston, Virginia: 2012
}

For more information on the USGS - the Federal source for science about the Earth, its natural and living resources, natural hazards, and the environment, visit http://www.usgs.gov or call 1-888-ASK-USGS.

For an overview of USGS information products, including maps, imagery, and publications, visit http://www.usgs.gov/pubprod

To order this and other USGS information products, visit http://store.usgs.gov

Any use of trade, firm, or product names is for descriptive purposes only and does not imply endorsement by the U.S. Government.

Although this information product, for the most part, is in the public domain, it also may contain copyrighted materials as noted in the text. Permission to reproduce copyrighted items must be secured from the copyright owner.

Suggested citation:

Solberg, P.A., Moore, Bryan, and Blacklock, T.D., 2012, Comparison of 2008-2009 water years and historical waterquality data, upper Gunnison River Basin, Colorado: U.S. Geological Survey Data Series 687, 85 p. 


\section{Contents}

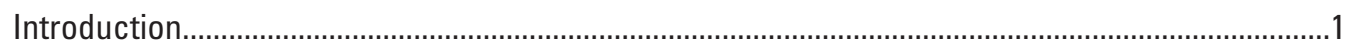

Definition of Terms

Water-Quality Sampling Sites ........................................................................................................

385224106590100 Coal Creek above Mouth near Crested Butte, Colorado ................................7

09111500 Slate River near Crested Butte, Colorado .............................................................13

385106106571000 Slate River above Baxter Gulch near Crested Butte, Colorado ...................19

09111700 Slate River above Squaw Gulch near Crested Butte, Colorado................................23

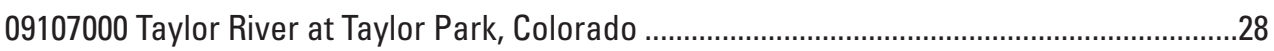

09112200 East River below Cement Creek, Colorado ...............................................................33

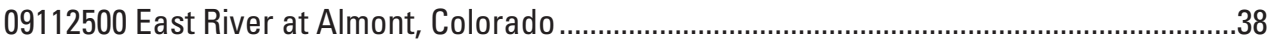

09113980 Ohio Creek above Mouth, near Gunnison, Colorado ................................................4

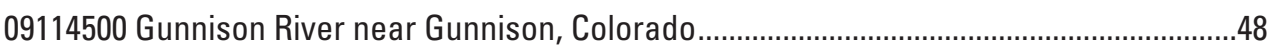

383126106475600 Tomichi Creek below Cochetopa Creek, Colorado .......................................53

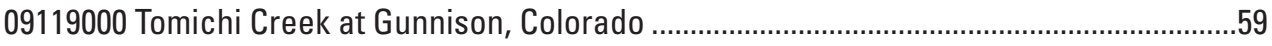

383103106594200 Gunnison River at County Road 32 below Gunnison, Colorado....................65

09123490 Lake Fork Gunnison River below Deadmans Gulch near Lake City, Colorado.........71

381633107054700 Cebolla Creek at Powderhorn, Colorado ....................................................76

385330106581401 Mt. Crested Butte Wastewater Treatment Plant Effluent .............................82

385229106583100 Crested Butte Wastewater Treatment Plant Effluent ...................................84

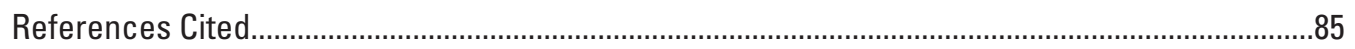

\section{Figures}

1. Location of sampling sites.........................................................................................

2. Time distribution and associated discharge of water-quality samples for Coal Creek above mouth near Crested Butte, Colorado .......................................................7

3. Time-series plots of selected water-quality constituents for Coal Creek above mouth near Crested Butte, Colorado ...................................................................

4. Time distribution and associated discharge of water-quality samples for Slate River near Crested Butte, Colorado.

5. Time-series plots of selected water-quality constituents for Slate River near Crested Butte, Colorado

6. Daily mean discharge and time distribution of water-quality samples for Slate River above Baxter Gulch, Colorado .

7. Time-series plots of selected water-quality constituents for Slate River above Baxter Gulch, Colorado.

8. Time distribution and associated discharge of water-quality samples for Slate River above Squaw Gulch, Colorado.

9. Time-series plots of selected water-quality constituents for Slate River above Squaw Gulch, Colorado

10. Daily mean discharge and time distribution of water-quality samples for Taylor River at Taylor Park, Colorado 
11. Time-series plots of selected water-quality constituents for Taylor River at Taylor Park, Colorado

12. Daily mean discharge and time distribution of water-quality samples for East River below Cement Creek, Colorado.

13. Time-series plots of selected water-quality constituents for East River below Cement Creek, Colorado.

14. Daily mean discharge and time distribution of water-quality samples for East River at Almont, Colorado.

15. Time-series plots of selected water-quality constituents for East River at Almont, Colorado

16. Daily mean discharge and time distribution of water-quality samples for Ohio Creek above mouth near Gunnison, Colorado

17. Time-series plots of selected water-quality constituents for Ohio Creek above mouth near Gunnison, Colorado.

18. Daily mean discharge and time distribution of water-quality samples for Gunnison River at Gunnison, Colorado

19. Time-series plots of selected water-quality constituents for Gunnison River at Gunnison, Colorado

20. Time distribution and associated discharge of water-quality samples for Tomichi Creek below Cochetopa Creek, Colorado

21. Time-series plots of selected water-quality constituents for Tomichi Creek below Cochetopa Creek, Colorado

22. Daily mean discharge and time distribution of water-quality samples for Tomichi Creek at Gunnison, Colorado

23. Time-series plots of selected water-quality constituents for Tomichi Creek at Gunnison, Colorado.

24. Time distribution and associated discharge of water-quality samples for Gunnison River at County Road 32 below Gunnison, Colorado

25. Time-series plots of selected water-quality constituents for Gunnison River at County Road 32 below Gunnison, Colorado

26. Time distribution and associated discharge of water-quality samples for Lake Fork Gunnison River below Deadmans Gulch, Colorado.

27. Time-series plots of selected water-quality constituents for Lake Fork Gunnison River below Deadmans Gulch, Colorado

28. Time distribution and associated discharge of water-quality samples for Cebolla Creek at Powderhorn, Colorado

29. Time-series plots of selected water-quality constituents for Cebolla Creek at Powderhorn, Colorado

30. Time-series plots of selected water-quality constituents for Mt. Crested Butte Wastewater Treatment Plant

31. Time-series plots of selected water-quality constituents for Crested Butte Wastewater Treatment Plant. 


\section{Tables}

1. Summary of individual station results .........................................................................

2. Summary of measured constituents and properties for Coal Creek above mouth at Crested Butte, Colorado, station 385224106590100 ................................................

3. Summary of seasonal Kendall trend test for Coal Creek above mouth at

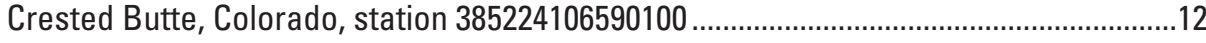

4. Summary of measured constituents and properties for Slate River near Crested Butte, Colorado, station 09111500

5. Summary of seasonal Kendall trend tests for Slate River near Crested Butte, Colorado, station 09111500 .

6. Summary of measured constituents and properties for Slate River above Baxter Gulch near Crested Butte, Colorado, station 385106106571000 ..

7. Summary of measured constituents and properties for Slate River above Squaw Gulch near Crested Butte, Colorado, station 09111700

8. Summary of measured constituents and properties for Taylor River at Taylor Park, Colorado, station 09107000.

9. Summary of measured constituents and properties for East River below Cement Creek, Colorado, station 09112200.

10. Summary of seasonal Kendall trend tests for East River below Cement Creek, Colorado, station 09112200

11. Summary of measured constituents and properties for East River at Almont, Colorado, station 09112500

12. Summary of seasonal Kendall trend tests for East River at Almont, Colorado, station 09112500 .

13. Time-series plots of selected water-quality constituents for Ohio Creek above mouth near Gunnison, Colorado, station 09113980

14. Summary of measured constituents and properties for Ohio Creek above mouth near Gunnison, Colorado, station 09113980

15. Summary of measured constituents and properties for Gunnison River near Gunnison, Colorado, station 09114500.

16. Summary of seasonal Kendall trend tests for Gunnison River near Gunnison, Colorado, station 09114500

17. Summary of measured constituents and properties for Tomichi Creek below Cochetopa Creek, Colorado.

18. Summary of seasonal Kendall trend tests for Tomichi Creek below Cochetopa Creek, Colorado, station 383126106475600

19. Summary of measured constituents and properties for Tomichi Creek at Gunnison, Colorado, station 09119000.

20. Summary of seasonal Kendall trend tests for Tomichi Creek at Gunnison, Colorado, station 09119000

21. Summary of measured constituents and properties for Gunnison River at CR 32 below Gunnison, Colorado, station 383103106594200

22. Summary of seasonal Kendall trend tests for Gunnison River at CR 32 below Gunnison, Colorado, station 383103106594200.

23. Summary of measured constituents and properties for Lake Fork Gunnison River below Deadmans Gulch, Colorado, station 09123490.

24. Summary of measured constituents and properties for Cebolla Creek at Powderhorn, Colorado, station 381633107054700. 
25. Summary of seasonal Kendall trend tests for Cebolla Creek at Powderhorn, Colorado, station 381633107054700

26. Summary of measured constituents and properties for Mt. Crested Butte

Wastewater Treatment Plant, station 385330106581401.

27. Summary of measured constituents and properties for Crested Butte

Wastewater Treatment Plant, station 385229106583100.

\section{Conversion Factors}

Inch/Pound to SI

\begin{tabular}{lcl}
\hline \multicolumn{1}{c}{ Multiply } & By & \multicolumn{1}{c}{ To obtain } \\
\hline inch & Length & \\
inch & 2.54 & centimeter $(\mathrm{cm})$ \\
foot $(\mathrm{ft})$ & 25.4 & millimeter $(\mathrm{mm})$ \\
mile $(\mathrm{mi})$ & 0.3048 & meter $(\mathrm{m})$ \\
& 1.609 & kilometer $(\mathrm{km})$ \\
\hline acre & Area & \\
square foot $\left(\mathrm{ft}^{2}\right)$ & 0.4047 & hectare $(\mathrm{ha})$ \\
square mile $\left(\mathrm{mi}^{2}\right)$ & 0.09290 & square meter $\left(\mathrm{m}^{2}\right)$ \\
square mile $\left(\mathrm{mi}^{2}\right)$ & 259.0 & hectare $(\mathrm{ha})$ \\
& 2.590 & square $\mathrm{kilometer}\left(\mathrm{km}^{2}\right)$ \\
\hline gallon $(\mathrm{gal})$ & Volume & \\
gallon $(\mathrm{gal})$ & 3.785 & liter $(\mathrm{L})$ \\
cubic foot $\left(\mathrm{ft}^{3}\right)$ & 0.003785 & cubic meter $\left(\mathrm{m}^{3}\right)$ \\
acre-foot $(\mathrm{acre}-\mathrm{ft})$ & 0.02832 & cubic meter $\left(\mathrm{m}^{3}\right)$ \\
& 1,233 & cubic meter $\left(\mathrm{m}^{3}\right)$ \\
\hline acre-foot per day $(\mathrm{acre}-\mathrm{ft} / \mathrm{d})$ & Flow rate & \\
cubic foot per second $\left(\mathrm{ft}{ }^{3} / \mathrm{s}\right)$ & 0.01427 & cubic meter per second $\left(\mathrm{m}^{3} / \mathrm{s}\right)$ \\
\hline
\end{tabular}

Temperature in degrees Celsius $\left({ }^{\circ} \mathrm{C}\right)$ may be converted to degrees Fahrenheit $\left({ }^{\circ} \mathrm{F}\right)$ as follows:

${ }^{\circ} \mathrm{F}=\left(1.8 \times^{\circ} \mathrm{C}\right)+32$

Temperature in degrees Fahrenheit $\left({ }^{\circ} \mathrm{F}\right)$ may be converted to degrees Celsius $\left({ }^{\circ} \mathrm{C}\right)$ as follows:

${ }^{\circ} \mathrm{C}=\left({ }^{\circ} \mathrm{F}-32\right) / 1.8$

Altitude, as used in this report, refers to distance above the vertical datum.

Specific conductance is given in microsiemens per centimeter at 25 degrees Celsius $(\mu \mathrm{S} / \mathrm{cm}$ at $\left.25^{\circ} \mathrm{C}\right)$.

Concentrations of chemical constituents in water are given either in milligrams per liter (mg/L) or micrograms per liter $(\mu \mathrm{g} / \mathrm{L})$. 


\section{Introduction}

Population growth and changes in land use have the potential to affect water quality and quantity in the upper Gunnison River Basin. In 1995, the U.S. Geological Survey (USGS), in cooperation with the Bureau of Land Management, City of Gunnison, Colorado River Water Conservation District, Crested Butte South Metropolitan District, Gunnison County, Hinsdale County, Mount Crested Butte Water and Sanitation District, National Park Service, Town of Crested Butte, U.S. Forest Service, Upper Gunnison River Water Conservancy District, and Western State College, established a water-quality monitoring program in the upper Gunnison River Basin to characterize current water-quality conditions and to assess the effects of increased urban development and other land-use changes on water quality. The monitoring network has evolved into two groups of sites: (1) sites that are considered long term and (2) sites that are considered rotational. Data from the long-term sites assist in defining temporal changes in water quality (how conditions may change over time). The rotational sites assist in the spatial definition of waterquality conditions (how conditions differ throughout the basin) and address local and short-term concerns. Biannual summaries of the water-quality data from the monitoring network provide a point of reference for stakeholder discussions regarding the location and purpose of water-quality monitoring sites in the upper Gunnison River Basin.

This report compares and summarizes the data collected during water years 2008 and 2009 to the historical data available at these sites. The introduction provides a map of the sampling sites, definitions of terms, and a one-page summary of selected water-quality conditions at the network sites. The remainder of the report is organized around the data collected at individual sites. Data collected during water years 2008 and 2009 are compared to historical data, State water-quality standards, and Federal water-quality guidelines. A seasonal Kendall test (Helsel and Hirsch, 1993) for trend analysis is completed when there is sufficient data (typically $>5$ years) at the station. Data were collected following USGS protocols (U.S. Geological Survey, variously dated). 


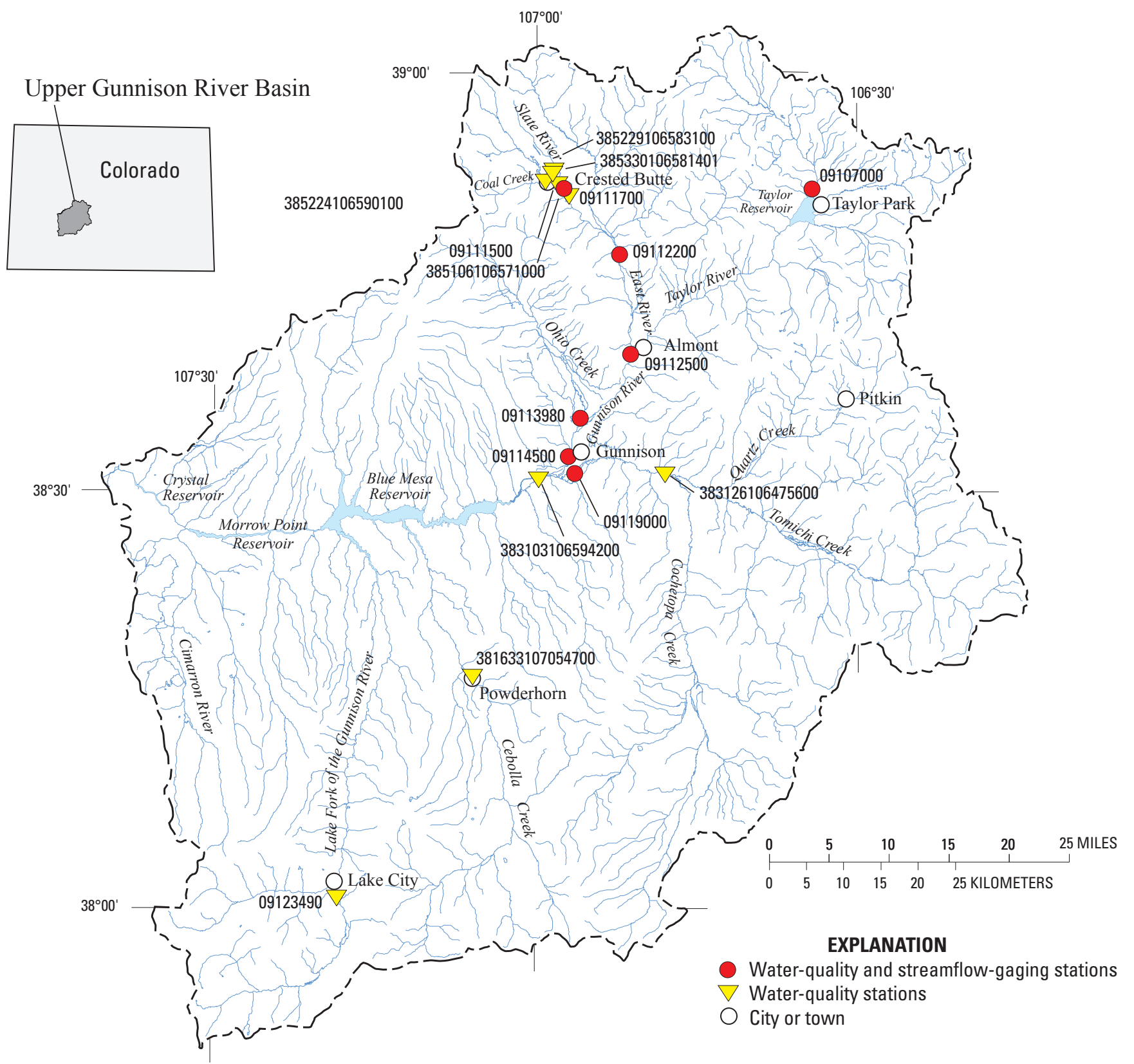

Figure 1. Location of sampling sites.

\begin{tabular}{llll}
\hline & \multicolumn{2}{c}{ Long-term sites } & \multicolumn{1}{c}{ Rotating stations } \\
\hline \multicolumn{1}{c}{ Site number } & \multicolumn{1}{c}{ Stations } & \multicolumn{1}{c}{ Site name } \\
\hline 385224106590100 & Coal Creek above mouth near Crested Butte & 385330106581401 & Mt. Crested Butte Wastewater Treament Plant \\
09111500 & Slate River near Crested Butte & 385229106583100 & Crested Butte Wastewater Treatment Plant \\
09112200 & East River below Cement Creek & 385106106571000 & Slate River above Baxter Gulch near Crested Butte \\
09112500 & East River at Almont & 09111700 & Slate River above Squaw Gulch near Crested Butte \\
09113980 & Ohio Creek above mouth near Gunnison & 09107000 & Taylor River at Taylor Park \\
09119000 & Tomichi Creek at Gunnison & 09114500 & Gunnison River near Gunnison \\
383103106594200 & Gunnison River at CR 32 below Gunnison & 383126106475600 & Tomichi Creek below Cochetopa Creek \\
& & 09123490 & Lake Fork below Deadmans Gulch near Lake City \\
& & 381633107054700 & Cebolla Creek at Powderhorn \\
\hline
\end{tabular}




\section{Definition of Terms}

Constituents results for which the State of Colorado has adopted a "chronic instream standard," or in the absence of that, for which the U. S. Environmental Protection Agency (USEPA) has published a "recommended level," are reported in terms of "concern levels" as defined below:

Low concern: The majority (85th percentile) of samples for a given site are below one-half the instream standard or recommended level. For example, if a constituent had a standard of 200 and the 85th percentile of the data was less than 100 it would be listed as low concern.

Medium concern: The 85th percentile of the data is between the instream standard and one-half the standard. This means that 15 percent or more of the data for a given site are nearing or are greater than the standard.

High concern: The 85th percentile of the data is above the instream standard or recommended level.

Cannot classify: The data cannot be placed into one of the three concern levels due to detection limits, no instream standard, or lack of samples.

The geometric mean is used for Escherichia coli rather than the 85th percentile. The concern level for the 2008-2009 water years are stated in the one-page summary and for each individual site. Temporary modifications to instream standards were not taken into account when calculating concern levels, but are footnoted when applicable. If a measured constituent is not listed with a concern level, it can be assumed that it fits into the 'Low concern' or 'Cannot classify' category.

The concern levels are consistent with the methods used by the State of Colorado to assess whether stream water-quality standards are being attained. The following is from the Unified Assessment Methodology, Water Quality Control Division, and was accessed October 2010 at http://www.cdphe.state.co.us/wq/Assessment/Assess_pdf/unified_assess_methodology_v1.pdf:

"1. Attainment of chronic chemical standards, in both lotic (streams and rivers) and limnic (lakes and reservoirs) systems, is based upon the 85th percentile of the ranked data, except as otherwise noted below. Percentile values are calculated by ranking individual data points in order of magnitude. Hardness based metal standards are evaluated by comparing the 85th percentile against the assigned hardness based equation using either the mean hardness at low flow or, when available, paired hardness and flow data. Total recoverable iron is evaluated against the median value, or the 50th percentile. Dissolved oxygen is evaluated at the 15th percentile. Minima $\mathrm{pH}$ is evaluated against the 15 th percentile, maxima at the 85th.

2. Acute standards are evaluated by comparison of raw values against the standard.

3. Sample data that are below detection limits will, in general, be treated as zeroes for assessment of attainment of chronic standards.

4. Attainment of coliform standards is assessed using the geometric mean. Notwithstanding the criterion at item 3 . above, coliform data which is reported as less than detect will be treated as a value of one to allow calculation of a geometric mean."

State standards for dissolved oxygen are based on minima, and for $\mathrm{pH}$, they are based on a range. The concern levels are defined as follows:

\section{Dissolved Oxygen Concern Levels:}

Low Concern: above $7 \mathrm{mg} / \mathrm{L}$

Medium Concern: between 6 and $7 \mathrm{mg} / \mathrm{L}$

High Concern: below $6 \mathrm{mg} / \mathrm{L}$ (below State instream standard)

\section{pH Concern Levels:}

Low Concern: between 7 and 8.5

Medium Concern: between 6.5 and 7 or 8.5 and 9.0

High Concern: outside the range of 6.5-9.0 (outside of State standards)

Hardness: A number of the trace elements have Table Value Standards (TVS), which are site-specific in-stream standards calculated using stream hardness. TVS equations use a stream hardness value calculated from the "lower 95th-percent confidence limit of the mean hardness value at the periodic low-flow criteria determined from a regression analysis of site-specific data" (Colorado Department of Public Health and Environment, 2010a). In this study for stream sampling sites, the annual low-flow 
period was defined as October through March. When there were more than five hardness values at a given sampling site during the low-flow period, the lower 95th-percent confidence limit of the mean hardness value was used in the TVS equation.

HUC: Hydrologic unit code-A geographic area representing part of or all of a surface drainage basin or distinct hydrologic feature. Each hydrologic unit is identified by an 8-digit number.

Censored value: A value reported as less than a laboratory reporting level, for example $<0.05$. Censored values are calculated in accordance with methods outlined by the State of Colorado, for computation of the statistics shown in the summary tables. Censored values, except for Escherichia coli are assumed to be zero, whereas Escherichia coli is assumed to be 1.

Laboratory Reporting Level (LRL): Generally is equal to twice the yearly determined long-term method detection level (LT-MDL). The LRL controls false-negative error. The probability of falsely reporting a nondetection for a sample that contained an analyte at a concentration equal to or greater than the LRL is predicted to be less than or equal to 1 percent. The value of the LRL will be reported with a "less than" $(<)$ remark code for samples in which the analyte was not detected. The USGS National Water Quality Laboratory collects quality-control data from selected analytical methods on a continuing basis to determine LT-MDLs and to establish LRLs. These values are reevaluated annually based on the most current quality-control data and therefore may change. (Note: Previously, the LRL has been called the nondetection value or NDV - a term which is no longer used.) For more information on LT-MDL's, refer to OFR 99-193 (Childress and others, 1999), available at the following link. http://nwql.usgs.gov/Public/rpt.shtml?OFR-99-193

Stream segment: (from Colorado Department of Public Health and Environment Water Quality Control, 2010b, Commission Regulation no. 31)

“(a) For purposes of adopting site-specific classifications and water quality standards, the streams and other surface water bodies shall be identified according to river basin and/or subbasin and specific water segments.

(b) Segments may constitute a specified stretch of a river mainstem, a specific tributary, a specific lake or reservoir, or a generally defined grouping of waters within the basin (e.g., a specific mainstem segment and all tributaries flowing into that mainstem segment.

(c) Segments shall generally be delineated according to the points at which the use, physical characteristics or water quality characteristics of a watercourse are determined to change significantly enough to require a change in use classifications and/or water quality standards. In many cases, such transition points can be specifically identified from available water quality data. In other cases, however, the delineation of segments shall be based upon best judgments of where instream changes in uses, physical characteristics or water quality occur, based upon upstream and downstream data."Water supply standard refers to waters with a water-supply classification, Colorado Department of Public Health and Environment (2010b), Water Quality Control Commission Regulation no. 31.11 (6)."

303 (d): (from Colorado Department of Public Health and Environment, Colorado's Section 303(d) Listing Methodology, May 2009, accessed at http://www.cdphe.state.co.us/op/wqcc/SpecialTopics/303(d)/303dLM2010.pdf

"Section 303(d) of the federal Clean Water Act requires states to identify waters where effluent limitations mandated by Section 301(b)(1)(A) and Section 301(b)(1)(B) are not stringent enough to attain water quality standards. These waters are compiled into the Section 303(d) list of impaired waters. The Colorado Section 303(d) List identifies those water bodies, which are impaired by one or more pollutants."

Dissolved and total: Constituent concentrations listed in the accompanying figures and tables refer to dissolved concentrations unless specifically stated otherwise. Dissolved constituent concentrations are derived from laboratory analysis of water samples filtered through a 0.45 micrometer filter, whereas total constituent concentrations are determined from laboratory analysis of unfiltered water samples.

Instantaneous Discharge is defined, for the purpose of this report, as the discharge of the river at logged intervals of time, computed using a stage-discharge relation. A common logged interval for the gaging stations in this basin is every 15 minutes.

Daily mean discharge is derived by summarizing time-series data of streamflow for each day for the period of record, and calculating the mean. The time-series data used to derive daily mean values in this report are typically collected at an interval of 15 minutes. 
Water Year is defined in this report as the 12-month period October 1 through September 30, designated by the calendar year in which it ends. (http://water.usgs.gov/nwc/explain_data.html)

Trend analyses were performed using the seasonal Kendall test (Helsel and Hirsch, 2002). This analysis measures the monotonic relation between constituent concentration and time. Because the analysis is rank-based, it is more resistant to the effects of small sample size, censored data, and non-normal population distributions. A trend was determined to be present when the p-value of the statistical test was less than the decision level (alpha-level) of 0.05 . Trends are identified as "up" when the Kendall tau is greater than zero, indicating that the concentrations increased more often than decreased over time. Trends are identified as "down" when the Kendall tau is less than zero, indicating that the concentration decreased more often than increased over time. A trend identified as "none" indicates there is insufficient evidence for rejection of the null hypothesis (p-value is greater than the decision level of 0.05 ), which states that no relation between concentration and time exists. 
[Al, aluminum ; Ca, calcium; $\mathrm{Cd}$, cadmium; $\mathrm{Cl}$, chloride; conductance, specific conductance; $\mathrm{Cu}$, copper; E. coli, Escherichia coli; $\mathrm{Fe}$, iron; $\mathrm{Mg}$, magnesium; Mn, manganese; nitrate, nitrite plus nitrate; Pb, lead; sediment, suspended sediment; $\mathrm{SO}_{4}$, sulfate; $\mathrm{Zn}$, zinc]

\begin{tabular}{|c|c|c|c|c|}
\hline Station name and number from figure 1 & $\begin{array}{l}\text { pH, Dissolved Oyxgen, } \\
\text { Temperature }\end{array}$ & E. Coli & $\begin{array}{l}\text { Trace elements/ } \\
\text { Metals }\end{array}$ & Trend \\
\hline $\begin{array}{l}\text { Coal Creek above mouth near Crested } \\
\text { Butte } 385224106590100\end{array}$ & Low concern & Low concern & $\begin{array}{l}\text { High concern: } \mathrm{Cd}, \mathrm{Zn} \\
\text { Medium concern: } \mathrm{Cu}\end{array}$ & $\begin{array}{l}\text { None: conductance, } \mathrm{pH} \text {, ammonia plus organic, nitrate, total } \\
\text { phosphorus, } \mathrm{Ca}, \mathrm{Mg}, \mathrm{Cd}, \mathrm{Cu}, \mathrm{Fe}, \mathrm{Mn}, \mathrm{Zn}, \mathrm{Al} \text {, sediment, } E \text {. coli }\end{array}$ \\
\hline Slate River near Crested Butte 09111500 & Low concern & Low concern & $\begin{array}{l}\text { High concern: } \mathrm{Cd}, \mathrm{Zn} \\
\text { Medium concern: } \mathrm{Cu}, \mathrm{Pb}\end{array}$ & $\begin{array}{l}\text { Up: conductance } \\
\text { Down: ammonia plus organic, } \mathrm{Mn} \\
\text { None: } \mathrm{pH} \text {, ammonia, nitrate, nitrite, total phosphorus, orthophos- } \\
\text { phate, } \mathrm{Ca}, \mathrm{Mg}, \mathrm{Cd}, \mathrm{Cu}, \mathrm{Fe}, \mathrm{Zn} \text {, sediment, E. coli }\end{array}$ \\
\hline $\begin{array}{l}\text { Slate River above Baxter Gulch near } \\
\text { Crested Butte } 385106106571000\end{array}$ & Low concern & Low concern & No data & Insufficient data \\
\hline $\begin{array}{l}\text { Slate River above Squaw Gulch near } \\
\text { Crested Butte } 09111700\end{array}$ & Low concern & Low concern & High concern: $\mathrm{Cd}, \mathrm{Zn}$ & Insufficient data \\
\hline Taylor River at Taylor Park 09107000 & Low concern & Low concern & Medium concern: $\mathrm{Fe}$ & Insufficient data \\
\hline $\begin{array}{l}\text { East River below Cement Creek } \\
\quad 09112200\end{array}$ & Low concern & Low concern & No data & $\begin{array}{l}\text { Up: total phosphorus } \\
\text { None: conductance, } \mathrm{pH} \text {, ammonia plus organic, nitrate, E. coli }\end{array}$ \\
\hline East River at Almont 09112500 & Low concern & Low concern & No data & $\begin{array}{l}\text { Up: conductance, total phosphorus } \\
\text { None: } \mathrm{pH} \text {, ammonia plus organic, nitrate, E. coli }\end{array}$ \\
\hline $\begin{array}{l}\text { Ohio Creek above mouth near Gunnison } \\
09113980\end{array}$ & Low concern & Low concern & No data & $\begin{array}{l}\text { None: conductance, } \mathrm{pH} \text {, ammonia plus organic, nitrate, total } \\
\text { phosphorus, orthophosphate, } E \text {. coli }\end{array}$ \\
\hline Gunnison River near Gunnison 09114500 & Low concern & Low concern & No data & $\begin{array}{l}\text { Up: conductance, ammonia plus organic } \\
\text { None: } \mathrm{pH} \text {, nitrate, total phosphorus, E. coli }\end{array}$ \\
\hline $\begin{array}{l}\text { Tomichi Creek below Cochetopa Creek } \\
383126106475600\end{array}$ & Low concern & Low concern & Low concern & $\begin{array}{l}\text { Down: conductance } \\
\text { None: } \mathrm{pH} \text {, ammonia plus organic, total phosphorus, orthophos- } \\
\text { phate, } \mathrm{Ca}, \mathrm{Mg}, \mathrm{Cu}, \mathrm{Fe}, \mathrm{Mn}, \mathrm{Zn} \text {, sediment, } E \text {. coli }\end{array}$ \\
\hline Tomichi Creek at Gunnison 09119000 & High concern: Temperature & Low concern & Low concern & $\begin{array}{l}\text { Up: } \mathrm{pH} \text {, ammonia plus organic, } \mathrm{Cu} \\
\text { None: conductance, total phosphorus, orthophosphate, } \mathrm{Ca}, \mathrm{Mg} \text {, } \\
\text { Fe, Mn, sediment, E. coli }\end{array}$ \\
\hline $\begin{array}{l}\text { Gunnison River at County Road } 32 \text { below } \\
\text { Gunnison } 383103106594200\end{array}$ & Low concern & Low concern & Low concern & $\begin{array}{l}\text { Up: conductance, } \mathrm{Ca}, \mathrm{Mg} \\
\text { None: } \mathrm{pH} \text {, ammonia plus organic, nitrate, total phosphorus, ortho- } \\
\text { phosphate, } \mathrm{Cu}, \mathrm{Fe}, \mathrm{Mn}, \mathrm{Zn} \text {, sediment } E \text {. coli }\end{array}$ \\
\hline $\begin{array}{l}\text { Lake Fork Gunnison River below } \\
\text { Deadmans Gulch } 09123490\end{array}$ & High concern: Temperature & Low concern & Low concern & Insufficient data \\
\hline $\begin{array}{l}\text { Cebolla Creek at Powderhorn } \\
\quad 381633107054700\end{array}$ & Low concern & Low concern & Low concern & $\begin{array}{l}\text { Down: } \mathrm{Ca}, \mathrm{Mg} \\
\text { None: conductance, } \mathrm{pH} \text {, ammonia plus organic, total phosphorus, } \\
\text { orthophosphate, } \mathrm{Mn}\end{array}$ \\
\hline
\end{tabular}




\section{Water-Quality Sampling Sites 385224106590100 Coal Creek above Mouth near Crested Butte, Colorado}

\section{Current Reason for Inclusion:}

To collect baseline data, characterize effects from mining, and facilitate remediation of the affected surface waters. Part of the rotational group of sites.

General Station Information:

Location:

Pedestrian bridge on Butte Avenue in Crested Butte.

Station Type:

USGS water quality

Period of Record:

Water quality: November 2000-September 2009

Latitude: 385224

Drainage area: Not determined

HUC: 14020001

Longitude: 1065901

Stream segment: 12

USGS Data Summary: Water Years 2008-2009:

General Chemistry:

$\mathrm{pH}$ : Low concern

Dissolved oxygen: Low concern

Temperature: Low concern

Nutrients:

Low concern

E. coli:

Low concern

Trace Elements/metals:

High concern: Cadmium and zinc

Medium concern: Copper

Other constituents of concern: None for constituents tested.

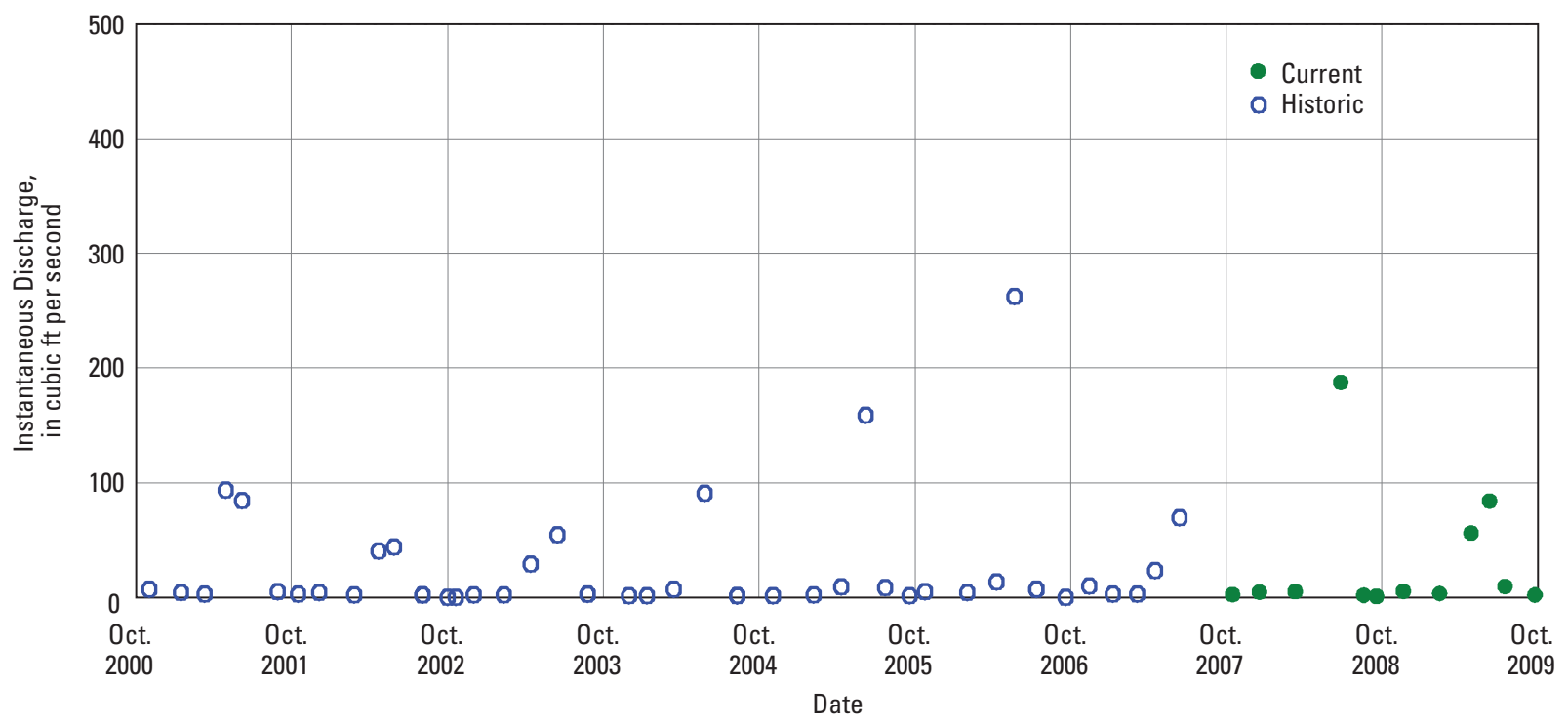

Figure 2. Time distribution and associated discharge of water-quality samples for Coal Creek above mouth near Crested Butte, Colorado. 

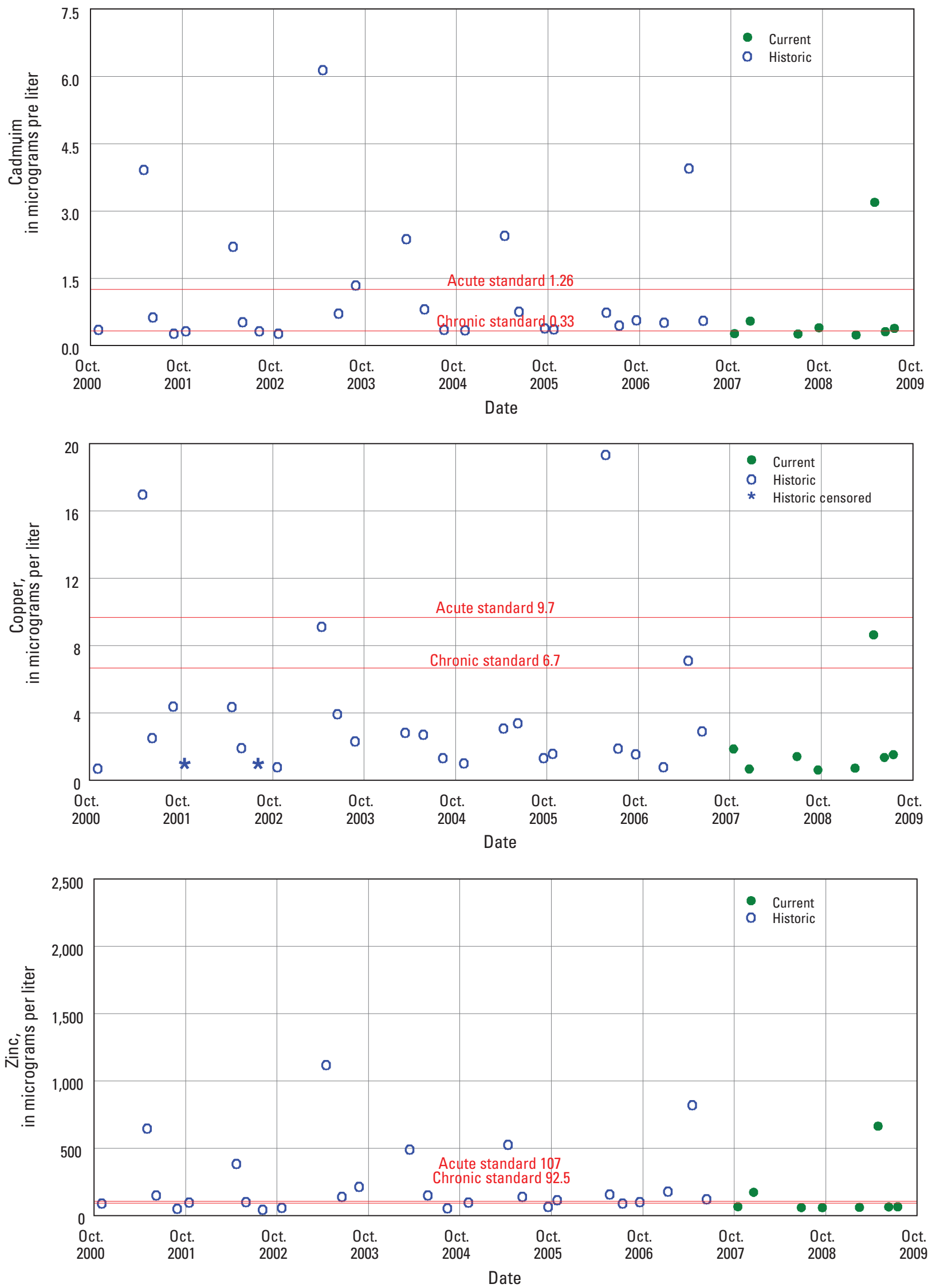

Figure 3. Time-series plots of selected water-quality constituents for Coal Creek above mouth near Crested Butte, Colorado. 
Table 2. Summary of measured constituents and properties for Coal Creek above mouth at Crested Butte, Colorado, station 385224106590100.

[--, no data or not applicable; L, low; M, medium; H, high; NTRU, Nephelometric Turbidity Ratio Unit; E. coli, Escherichia coli; mg/m² milligrams per square meter; USEPA, U.S. Environmental Protection Agency; CDPHE, Colorado Department of Public Health and Environment; Cd(ch), Cadmium, chronic standard; Zn(ch), Zinc, chronic standard]

\begin{tabular}{|c|c|c|c|c|c|c|c|c|c|c|c|c|c|c|}
\hline $\begin{array}{l}\text { Property } \\
\text { or } \\
\text { constituent }\end{array}$ & $\begin{array}{l}\text { Period } \\
\text { (water } \\
\text { years) }\end{array}$ & $\begin{array}{l}\text { Number } \\
\text { of } \\
\text { samples }\end{array}$ & $\begin{array}{c}\text { Number } \\
\text { of } \\
\text { censored } \\
\text { values }\end{array}$ & Median' & Maximum & $\begin{array}{c}\text { Date } \\
\text { of } \\
\text { Maximum }\end{array}$ & $\begin{array}{c}\text { 15th } \\
\text { percentile }\end{array}$ & $\begin{array}{c}\text { 85th } \\
\text { percentile }\end{array}$ & $\begin{array}{c}\text { Geometric } \\
\text { Mean }^{2}\end{array}$ & $\begin{array}{c}\text { Standard } \\
\text { or } \\
\text { Chronic } \\
\text { standard }^{3}\end{array}$ & $\begin{array}{l}\text { Number of } \\
\text { exceedences } \\
\text { Standard or } \\
\text { Chronic }\end{array}$ & $\begin{array}{l}\text { Acute } \\
\text { standard }\end{array}$ & $\begin{array}{c}\begin{array}{c}\text { Number } \\
\text { of } \\
\text { exceedences } \\
\text { Acute }\end{array} \\
\end{array}$ & $\begin{array}{l}\text { Level } \\
\text { of } \\
\text { concern }\end{array}$ \\
\hline $\begin{array}{l}\text { Instantaneous discharge, in cubic } \\
\text { feet per second }\end{array}$ & 2001-2007 & 44 & 0 & 4.6 & 263 & $05 / 23 / 06$ & 1.9 & 58.6 & -- & -- & -- & -- & -- & -- \\
\hline $\begin{array}{l}\text { Instantaneous discharge, in cubic } \\
\text { feet per second }\end{array}$ & 2008-2009 & 12 & 0 & 5.2 & 188 & $06 / 26 / 08$ & 2.2 & 89.7 & -- & -- & -- & -- & -- & -- \\
\hline Turbidity, in NTRU & 2001-2007 & 25 & 6 & 2.4 & 43 & 05/01/01 & 0.0 & 6.6 & -- & -- & -- & -- & -- & -- \\
\hline Turbidity, in NTRU & 2008-2009 & 8 & 5 & 0.0 & 8.9 & 04/28/09 & 0.0 & 6.9 & -- & -- & -- & -- & -- & -- \\
\hline $\begin{array}{l}\text { Dissolved oxygen, in milligrams } \\
\text { per liter }\end{array}$ & 2001-2007 & 42 & 0 & 9.5 & 11.3 & $02 / 01 / 06$ & 7.4 & 10.2 & -- & 6.0 & 0 & -- & -- & $\mathrm{L}$ \\
\hline $\begin{array}{l}\text { Dissolved oxygen, in milligrams } \\
\text { per liter }\end{array}$ & 2008-2009 & 12 & 0 & 9.3 & 11.3 & $12 / 18 / 07$ & 8.1 & 10.5 & -- & 6.0 & 0 & -- & -- & $\mathrm{L}$ \\
\hline $\mathrm{pH}$, in standard units & 2001-2007 & 42 & 0 & 7.8 & 8.3 & $11 / 04 / 04$ & 7.4 & 8.0 & -- & 6.5 & 0 & 9.0 & -- & $\mathrm{L}$ \\
\hline $\mathrm{pH}$, in standard units & 2008-2009 & 12 & 0 & 7.9 & 8.1 & $10 / 16 / 07$ & 7.2 & 8.0 & -- & 6.5 & 0 & 9.0 & -- & $\mathrm{L}$ \\
\hline $\begin{array}{l}\text { Specific conductance, in microsie- } \\
\text { mens per centimeter }\end{array}$ & 2001-2007 & 42 & 0 & 204 & 365 & $02 / 11 / 03$ & 78 & 304 & -- & -- & -- & -- & -- & -- \\
\hline $\begin{array}{l}\text { Specific conductance, in microsie- } \\
\text { mens per centimeter }\end{array}$ & 2008-2009 & 12 & 0 & 206 & 375 & 09/18/08 & 65 & 370 & -- & -- & -- & -- & -- & -- \\
\hline Temperature, degrees Celsius & 2001-2007 & 42 & 0 & 3.4 & 16.3 & $08 / 05 / 02$ & 0.0 & 12.3 & -- & 17.0 & 0 & -- & -- & $\mathrm{L}$ \\
\hline Temperature, degrees Celsius & 2008-2009 & 12 & 0 & 5.3 & 13.5 & 07/16/09 & 0.2 & 10.1 & -- & 17.0 & 0 & -- & -- & $\mathrm{L}$ \\
\hline Calcium, in milligrams per liter & 2001-2007 & 26 & 0 & 18.7 & 55.4 & $03 / 15 / 04$ & 8.83 & 43.4 & -- & -- & -- & -- & -- & -- \\
\hline Calcium, in milligrams per liter & 2008-2009 & 8 & 0 & 24.7 & 58.0 & 09/18/08 & 7.75 & 47.1 & -- & -- & -- & -- & -- & -- \\
\hline Magnesium, in milligrams per liter & 2001-2007 & 26 & 0 & 2.75 & 4.79 & $08 / 05 / 02$ & 1.14 & 4.20 & -- & -- & -- & -- & -- & -- \\
\hline Magnesium, in milligrams per liter & 2008-2009 & 8 & 0 & 2.71 & 3.81 & 09/18/08 & 1.05 & 3.78 & -- & -- & -- & -- & -- & -- \\
\hline Chloride, in milligrams per liter & 2009 & 5 & 0 & 1.94 & 3.93 & $04 / 28 / 09$ & 0.61 & 3.93 & -- & -- & -- & -- & -- & -- \\
\hline $\begin{array}{l}\text { Ammonia plus organic nitrogen, in } \\
\text { milligrams per liter }\end{array}$ & 2001-2007 & 40 & 9 & 0.088 & 0.374 & $05 / 23 / 06$ & 4 & 0.140 & -- & -- & -- & -- & -- & -- \\
\hline $\begin{array}{l}\text { Ammonia plus organic nitrogen, in } \\
\text { milligrams per liter }\end{array}$ & 2008-2009 & 11 & 3 & 0.078 & 0.171 & 06/10/09 & 4 & 0.162 & -- & -- & -- & -- & -- & -- \\
\hline $\begin{array}{l}\text { Ammonia nitrogen, in milligrams } \\
\text { per liter }\end{array}$ & 2001-2007 & 40 & 28 & 4 & 0.02 & $03 / 15 / 04$ & 4 & 0.01 & -- & 3.57 & 0 & 10.3 & 0 & $\mathrm{~L}$ \\
\hline $\begin{array}{l}\text { Ammonia nitrogen, in milligrams } \\
\text { per liter }\end{array}$ & 2008-2009 & 11 & 9 & 4 & 0.01 & $12 / 18 / 07$ & 4 & 0.01 & -- & 3.78 & 0 & 11.7 & 0 & $\mathrm{~L}$ \\
\hline $\begin{array}{l}\text { Nitrite plus nitrate nitrogen, in } \\
\text { milligrams per liter }\end{array}$ & $2001-2007$ & 40 & 5 & 0.043 & 0.494 & $04 / 15 / 03$ & 0.006 & 0.097 & -- & 10.0 & 0 & -- & -- & $\mathrm{L}$ \\
\hline $\begin{array}{l}\text { Nitrite plus nitrate nitrogen, in } \\
\text { milligrams per liter }\end{array}$ & 2008-2009 & 11 & 2 & 0.015 & 0.124 & 04/28/09 & 4 & 0.085 & -- & 10.0 & 0 & -- & -- & $\mathrm{L}$ \\
\hline $\begin{array}{l}\text { Nitrite nitrogen, in milligrams } \\
\text { per liter }\end{array}$ & 2001-2007 & 40 & 31 & 4 & 0.002 & $12 / 06 / 01$ & 4 & 0.001 & -- & 0.05 & 0 & -- & -- & $\mathrm{L}$ \\
\hline
\end{tabular}


Table 2. Summary of measured constituents and properties for Coal Creek above mouth at Crested Butte, Colorado, station 385224106590100.-Continued

[--, no data or not applicable; L, low; M, medium; H, high; NTRU, Nephelometric Turbidity Ratio Unit; E. coli, Escherichia coli; mg/m², milligrams per square meter; USEPA, U.S. Environmental Protection Agency; CDPHE, Colorado Department of Public Health and Environment; Cd(ch), Cadmium, chronic standard; Zn(ch), Zinc, chronic standard]

\begin{tabular}{|c|c|c|c|c|c|c|c|c|c|c|c|c|c|c|}
\hline $\begin{array}{c}\text { Property } \\
\text { or } \\
\text { constituent }\end{array}$ & $\begin{array}{l}\text { Period } \\
\text { (water } \\
\text { years) }\end{array}$ & $\begin{array}{c}\text { Number } \\
\text { of } \\
\text { samples }\end{array}$ & $\begin{array}{c}\begin{array}{c}\text { Number } \\
\text { of }\end{array} \\
\text { censored } \\
\text { values }\end{array}$ & Median' & Maximum & $\begin{array}{c}\text { Date } \\
\text { of } \\
\text { Maximum }\end{array}$ & $\begin{array}{c}\text { 15th } \\
\text { percentile }\end{array}$ & $\begin{array}{c}\text { 85th } \\
\text { percentile }\end{array}$ & $\begin{array}{c}\text { Geometric } \\
\text { Mean }^{2}\end{array}$ & $\begin{array}{l}\text { Standard } \\
\text { or } \\
\text { Chronic } \\
\text { standard } \\
\end{array}$ & $\begin{array}{c}\text { Number of } \\
\text { exceedences } \\
\text { Standard or } \\
\text { Chronic }\end{array}$ & $\begin{array}{l}\text { Acute } \\
\text { standard }\end{array}$ & $\begin{array}{c}\begin{array}{c}\text { Number } \\
\text { of } \\
\text { exceedences } \\
\text { Acute }\end{array} \\
\end{array}$ & $\begin{array}{c}\text { Level } \\
\text { of } \\
\text { concern }\end{array}$ \\
\hline $\begin{array}{l}\text { Nitrite nitrogen, in milligrams } \\
\text { per liter }\end{array}$ & $2008-2009$ & 11 & 11 & 4 & ${ }^{5}<0.002$ & -- & 4 & 4 & -- & 0.05 & 0 & -- & -- & $\mathrm{L}$ \\
\hline $\begin{array}{l}\text { Orthophosphate, in milligrams } \\
\text { per liter }\end{array}$ & 2001-2007 & 40 & 36 & 4 & ${ }^{5}<0.012$ & -- & 4 & 4 & -- & -- & -- & -- & -- & -- \\
\hline $\begin{array}{l}\text { Orthophosphate, in milligrams } \\
\text { per liter }\end{array}$ & 2008-2009 & 11 & 7 & 4 & ${ }^{5}<0.008$ & -- & 4 & 4 & -- & -- & -- & -- & -- & -- \\
\hline $\begin{array}{l}\text { Phosphorus (total), in milligrams } \\
\text { per liter }\end{array}$ & $2001-2007$ & 40 & 5 & 0.006 & 0.033 & 05/23/06 & 0.002 & 0.012 & -- & -- & -- & -- & -- & -- \\
\hline $\begin{array}{l}\text { Phosphorus (total), in milligrams } \\
\text { per liter }\end{array}$ & 2008-2009 & 12 & 3 & 0.005 & 0.013 & 04/28/09 & 4 & 0.010 & -- & -- & -- & -- & -- & -- \\
\hline $\begin{array}{l}\text { Organic carbon, in milligrams } \\
\text { per liter }\end{array}$ & $2002-2007$ & 4 & 0 & 1.6 & 4.3 & $06 / 13 / 07$ & 1.1 & 4.3 & -- & -- & -- & -- & -- & -- \\
\hline $\begin{array}{l}\text { Organic carbon, in milligrams } \\
\text { per liter }\end{array}$ & 2008-2009 & 8 & 0 & 1.6 & 4.9 & 06/26/08 & 0.9 & 4.5 & -- & -- & -- & -- & -- & -- \\
\hline $\begin{array}{l}\text { E. coli, in colonies per } 100 \text { mil- } \\
\text { liliter }\end{array}$ & $2001-2007$ & 39 & 10 & 1 & 22 & 07/25/05 & 1 & 6 & 2.4 & 126 & 0 & -- & -- & $\mathrm{L}$ \\
\hline $\begin{array}{l}\text { E. coli, in colonies per } 100 \text { mil- } \\
\text { liliter }\end{array}$ & 2008-2009 & 12 & 2 & 2 & 73 & 08/19/08 & 1 & 6 & -- & 126 & 0 & -- & -- & $\mathrm{L}$ \\
\hline $\begin{array}{l}\text { Biomass periphyton, ashfree dry- } \\
\text { mass, in grams per square meter }\end{array}$ & $2002-2006$ & 5 & 3 & 4 & 10.5 & 08/05/02 & 4 & 10.5 & -- & -- & -- & -- & -- & -- \\
\hline $\begin{array}{l}\text { Biomass periphyton, ashfree dry- } \\
\text { mass, in grams per square meter }\end{array}$ & 2008-2009 & 2 & 2 & 4 & ${ }^{5}<0.1$ & -- & 4 & 4 & -- & -- & -- & -- & -- & -- \\
\hline $\begin{array}{l}\text { Periphyton, biomass, ash weight, } \\
\text { in grams per square meter }\end{array}$ & $2002-2006$ & 5 & 0 & 576 & 858 & $08 / 26 / 03$ & 253 & 858 & -- & -- & -- & -- & -- & -- \\
\hline $\begin{array}{l}\text { Periphyton, biomass, ash weight, } \\
\text { in grams per square meter }\end{array}$ & 2008-2009 & 2 & 0 & 242 & 325 & 09/24/09 & 159 & 325 & -- & -- & -- & -- & -- & -- \\
\hline $\begin{array}{l}\text { Periphyton, biomass, dry weight, } \\
\text { in grams per square meter }\end{array}$ & 2002-2006 & 5 & 0 & 586 & 875 & $08 / 26 / 03$ & 261 & 875 & -- & -- & -- & -- & -- & -- \\
\hline $\begin{array}{l}\text { Periphyton, biomass, dry weight, } \\
\text { in grams per square meter }\end{array}$ & 2008-2009 & 2 & 0 & 245 & 329 & 09/24/09 & 160 & 329 & -- & -- & -- & -- & -- & -- \\
\hline $\begin{array}{l}\text { Chlorophyll a, periphyton, chromo- } \\
\text { fluoro, in } \mathrm{mg} / \mathrm{m}^{2}\end{array}$ & 2002-2006 & 5 & 0 & 3.95 & 7.13 & 08/05/02 & 2.29 & 7.13 & -- & -- & -- & -- & -- & -- \\
\hline $\begin{array}{l}\text { Chlorophyll a, periphyton, chromo- } \\
\text { fluoro, in } \mathrm{mg} / \mathrm{m}^{2}\end{array}$ & 2008-2009 & 2 & 0 & 2.64 & 4.96 & 09/24/09 & 0.32 & 4.96 & -- & -- & -- & -- & -- & -- \\
\hline Pheophytin a, periphyton, in $\mathrm{mg} / \mathrm{m}^{2}$ & $2002-2006$ & 5 & 0 & 1.4 & 3.1 & $08 / 05 / 02$ & 0.4 & 3.1 & -- & -- & -- & -- & -- & -- \\
\hline Pheophytin a, periphyton, in $\mathrm{mg} / \mathrm{m}^{2}$ & 2008-2009 & 2 & 0 & 0.7 & 1.3 & 09/24/09 & 0.1 & 1.3 & -- & -- & -- & -- & -- & -- \\
\hline Aluminum, in micrograms per liter & 2001-2007 & 26 & 0 & 38.9 & 241 & $05 / 01 / 01$ & 16.9 & 80.5 & -- & -- & -- & -- & -- & -- \\
\hline Aluminum, in micrograms per liter & $2008-2009$ & 8 & 0 & 38.8 & 307 & 04/28/09 & 25.1 & 227 & -- & -- & -- & -- & -- & -- \\
\hline Cadmium, in micrograms per liter & 2001-2007 & 26 & 0 & 0.57 & 6.15 & $04 / 15 / 03$ & 0.33 & 2.45 & -- & ${ }^{7} 0.33$ & 22 & 1.26 & 7 & $\mathrm{H}$ \\
\hline
\end{tabular}


Table 2. Summary of measured constituents and properties for Coal Creek above mouth at Crested Butte, Colorado, station 385224106590100.-Continued

[--, no data or not applicable; L, low; M, medium; H, high; NTRU, Nephelometric Turbidity Ratio Unit; E. coli, Escherichia coli; mg/m² milligrams per square meter; USEPA, U.S. Environmental Protection Agency; CDPHE, Colorado Department of Public Health and Environment; Cd(ch), Cadmium, chronic standard; Zn(ch), Zinc, chronic standard]

\begin{tabular}{|c|c|c|c|c|c|c|c|c|c|c|c|c|c|c|}
\hline $\begin{array}{c}\text { Property } \\
\text { or } \\
\text { constituent }\end{array}$ & $\begin{array}{l}\text { Period } \\
\text { (water } \\
\text { years) }\end{array}$ & $\begin{array}{l}\text { Number } \\
\text { of } \\
\text { samples }\end{array}$ & $\begin{array}{c}\text { Number } \\
\text { of } \\
\text { censored } \\
\text { values }\end{array}$ & Median' & Maximum & $\begin{array}{c}\text { Date } \\
\text { of } \\
\text { Maximum }\end{array}$ & $\begin{array}{c}\text { 15th } \\
\text { percentile }\end{array}$ & $\begin{array}{c}\text { 85th } \\
\text { percentile }\end{array}$ & $\begin{array}{l}\text { Geometric } \\
\text { Mean }^{2}\end{array}$ & $\begin{array}{c}\text { Standard } \\
\text { or } \\
\text { Chronic } \\
\text { standard }^{3}\end{array}$ & $\begin{array}{c}\text { Number of } \\
\text { exceedences } \\
\text { Standard or } \\
\text { Chronic }\end{array}$ & $\begin{array}{l}\text { Acute } \\
\text { standard }\end{array}$ & $\begin{array}{c}\begin{array}{c}\text { Number } \\
\text { of } \\
\text { exceedences } \\
\text { Acute }\end{array} \\
\end{array}$ & $\begin{array}{l}\text { Level } \\
\text { of } \\
\text { concern }\end{array}$ \\
\hline Cadmium, in micrograms per liter & 2008-2009 & 8 & 0 & 0.35 & 3.20 & $04 / 28 / 09$ & 0.25 & 2.27 & -- & ${ }^{7} 0.33$ & 4 & 1.26 & 1 & $\mathrm{H}$ \\
\hline Copper, in micrograms per liter & 2001-2007 & 26 & 2 & 2.4 & 19.3 & 05/23/06 & 0.77 & 7.0 & -- & 6.7 & 4 & 9.7 & 2 & $\mathrm{H}$ \\
\hline Copper, in micrograms per liter & 2008-2009 & 8 & 0 & 1.4 & 8.6 & $04 / 28 / 09$ & 0.64 & 6.3 & -- & 6.7 & 1 & 9.7 & 0 & M \\
\hline Iron, in micrograms per liter & $2001-2007$ & 26 & 2 & 14 & 70 & 05/01/01 & 5 & 39 & -- & -- & -- & -- & -- & -- \\
\hline Iron, in micrograms per liter & 2008-2009 & 8 & 0 & 15 & 79 & $04 / 28 / 09$ & 7 & 68 & -- & -- & -- & -- & -- & -- \\
\hline Lead, in micrograms per liter & $2001-2007$ & 26 & 15 & 4 & 0.91 & $06 / 07 / 01$ & 4 & 0.63 & -- & 1.72 & 0 & 44.2 & 0 & $\mathrm{~L}$ \\
\hline Lead, in micrograms per liter & 2008-2009 & 8 & 2 & 0.13 & 0.72 & $04 / 28 / 09$ & 4 & 0.56 & -- & 1.72 & 0 & 44.2 & 0 & $\mathrm{~L}$ \\
\hline Manganese, in micrograms per liter & 2001-2007 & 26 & 0 & 33.2 & 598 & $04 / 15 / 03$ & 15.8 & 277 & -- & 1,470 & 0 & 2,660 & 0 & $\mathrm{~L}$ \\
\hline Manganese, in micrograms per liter & 2008-2009 & 8 & 0 & 12.6 & 243 & $04 / 28 / 09$ & 8.1 & 172 & -- & 1,470 & 0 & 2,660 & 0 & $\mathrm{~L}$ \\
\hline Silver, in micrograms per liter & 2001-2007 & 26 & 25 & 4 & ${ }^{5}<0.3$ & -- & 4 & 4 & -- & 0.04 & 1 & 1.12 & 0 & $\mathrm{~L}$ \\
\hline Silver, in micrograms per liter & 2008-2009 & 8 & 5 & 4 & ${ }^{s}<0.1$ & -- & 4 & 4 & -- & 0.04 & 0 & 1.12 & 0 & $\mathrm{~L}$ \\
\hline Zinc, in micrograms per liter & 2001-2007 & 26 & 0 & 132 & 1,120 & $04 / 15 / 03$ & 57.2 & 525 & -- & 792.5 & 19 & 107 & 15 & $\mathrm{H}$ \\
\hline Zinc, in micrograms per liter & 2008-2009 & 8 & 0 & 65.6 & 666 & $04 / 28 / 09$ & 60.0 & 494 & -- & 792.5 & 2 & 107 & 2 & $\mathrm{H}$ \\
\hline $\begin{array}{l}\text { Suspended sediment, in milligrams } \\
\text { per liter }\end{array}$ & 2001-2007 & 25 & 2 & 2.9 & 25.0 & $05 / 23 / 06$ & 1.0 & 11.7 & -- & -- & -- & -- & -- & -- \\
\hline $\begin{array}{l}\text { Suspended sediment, in milligrams } \\
\text { per liter }\end{array}$ & 2008-2009 & 8 & 0 & 1.0 & 5.0 & $04 / 28 / 09$ & 1.0 & 4.6 & -- & -- & -- & -- & -- & -- \\
\hline
\end{tabular}

per liter

Censored values were replaced with 0 to compute median and 85 th percentiles (coliform censored values replaced with 1) see definitions section.

${ }^{2}$ Geometric mean listed for E. coli. Geometric mean computed for combined period of record (2001-2009).

${ }^{3}$ Colorado Department of Public Health and Environment, classification and numeric standards for Gunnison and Lower Dolores River Basins (2010a); and U.S. Environmental Protection Agency National Recommended Water Quality Criteria (2009).

${ }^{4}$ Median values and percentiles were not tabled when they were calculated as zero.

${ }^{5}$ All values below laboratory reporting level (LRL), current LRL used for current period maximum, highest LRL used for historic period.

${ }^{6}$ Use geometric mean for comparison to standard.

${ }^{7}$ Temporary modifications to CDPHE numeric standards for this stream segment: $\mathrm{Cd}(\mathrm{ch})=2.3$ and $\mathrm{Zn}(\mathrm{ch})=518$. Expiration Date of 12/31/2012. 


\section{Comparison of 2008-2009 Water Years and Historical Water-Quality Data, Upper Gunnison River Basin, Colorado}

Table 3. Summary of seasonal Kendall trend test for Coal Creek above mouth at Crested Butte, Colorado, station 385224106590100 .

[E.coli, Escherichia coli $]$

\begin{tabular}{lcccc}
\hline \multicolumn{1}{c}{ Property or constituent } & $\begin{array}{c}\text { Period } \\
\text { (water years) }\end{array}$ & Kendall tau $(\tau)$ & p-Value & Trend direction \\
\hline Specific conductance, in microsiemens per centimeter & $2001-2009$ & 0.059 & 0.705 & None \\
pH, in standard units & $2001-2009$ & -0.103 & 0.480 & None \\
Ammonia plus organic nitrogen, in milligrams per liter & $2001-2009$ & 0.103 & 0.460 & None \\
Nitrite plus nitrate nitrogen, in milligrams per liter & $2001-2009$ & 0.096 & 0.514 & None \\
Phosphorus (total), in milligrams per liter & $2001-2009$ & 0.029 & 0.611 & None \\
Calcium, in milligrams per liter & $2001-2009$ & -0.098 & 0.276 & None \\
Magnesium, in milligrams per liter & $2001-2009$ & -0.195 & 0.716 & None \\
Cadmium, in micrograms per liter & $2001-2009$ & 0.073 & 0.827 & None \\
Copper, in micrograms per liter & $2001-2009$ & -0.049 & 0.167 & None \\
Iron, in micrograms per liter & $2004-2009$ & 0.355 & 0.127 & None \\
Manganese, in micrograms per liter & $2001-2009$ & -0.268 & 0.146 & 0.513 \\
Zinc, in micrograms per liter & $2001-2009$ & 0.122 & 1.000 & None \\
Aluminum, in micrograms per liter & $2001-2009$ & 0.000 & 0.368 & None \\
Suspended sediment, in milligrams per liter & $2001-2009$ & $2001-2009$ & 0.118 & None \\
E. coli, in colonies per 100 milliliter & & &
\end{tabular}




\section{Slate River near Crested Butte, Colorado}

Current Reason for Inclusion: Long-term monitoring. This station characterizes water quality downstream from Crested Butte. The streamflow gage was moved downstream of State Highway 135 bridge in September 2006 to station 385106106571000 , Slate River above Baxter Gulch.

General Station Information: Location:

Station Type:

Period of Record:

Streamflow gaging:

Latitude: 385211

Longitude: 1065808
$400 \mathrm{ft}$ downstream from Washington Gulch (1 mi east of Crested Butte and $6.3 \mathrm{mi}$ upstream from mouth).

USGS water-quality and streamflow gaging

Water quality: March 1995-September 2009

April 1940-September 1951

October 1993-September 2006 (discontinued)

Drainage area: $68.9 \mathrm{mi}^{2}$

HUC: 14020002

USGS Data Summary: Water Years 2008-2009:

General Chemistry:

pH: Low concern

Dissolved oxygen: Low concern

Temperature: Low concern

Nutrients:

Low concern

E. coli:

Low concern

Trace Elements/metals:

High concern: Cadmium and zinc

Medium concern: Copper and lead

Other constituents of concern: None for constituents tested.

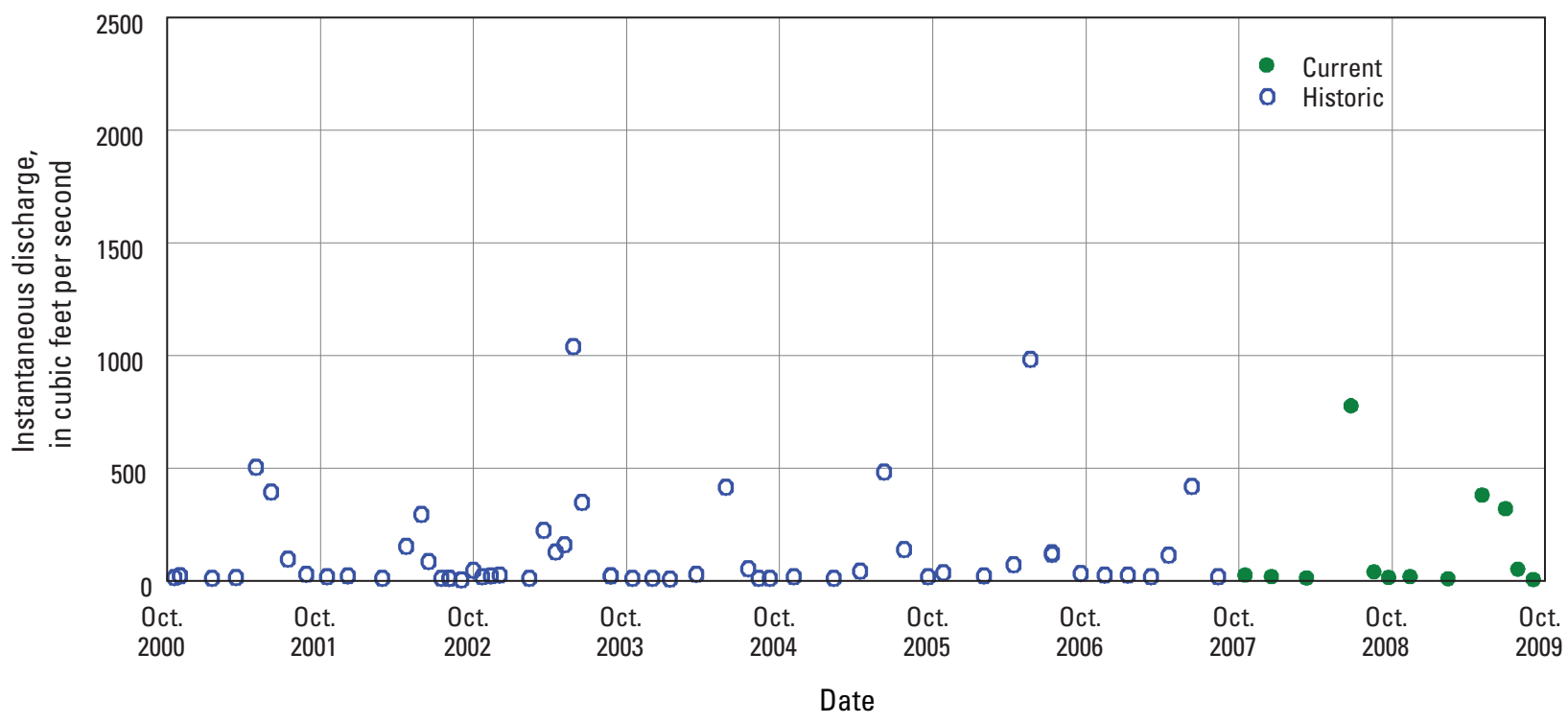

Figure 4. Time distribution and associated discharge of water-quality samples for Slate River near Crested Butte, Colorado. 

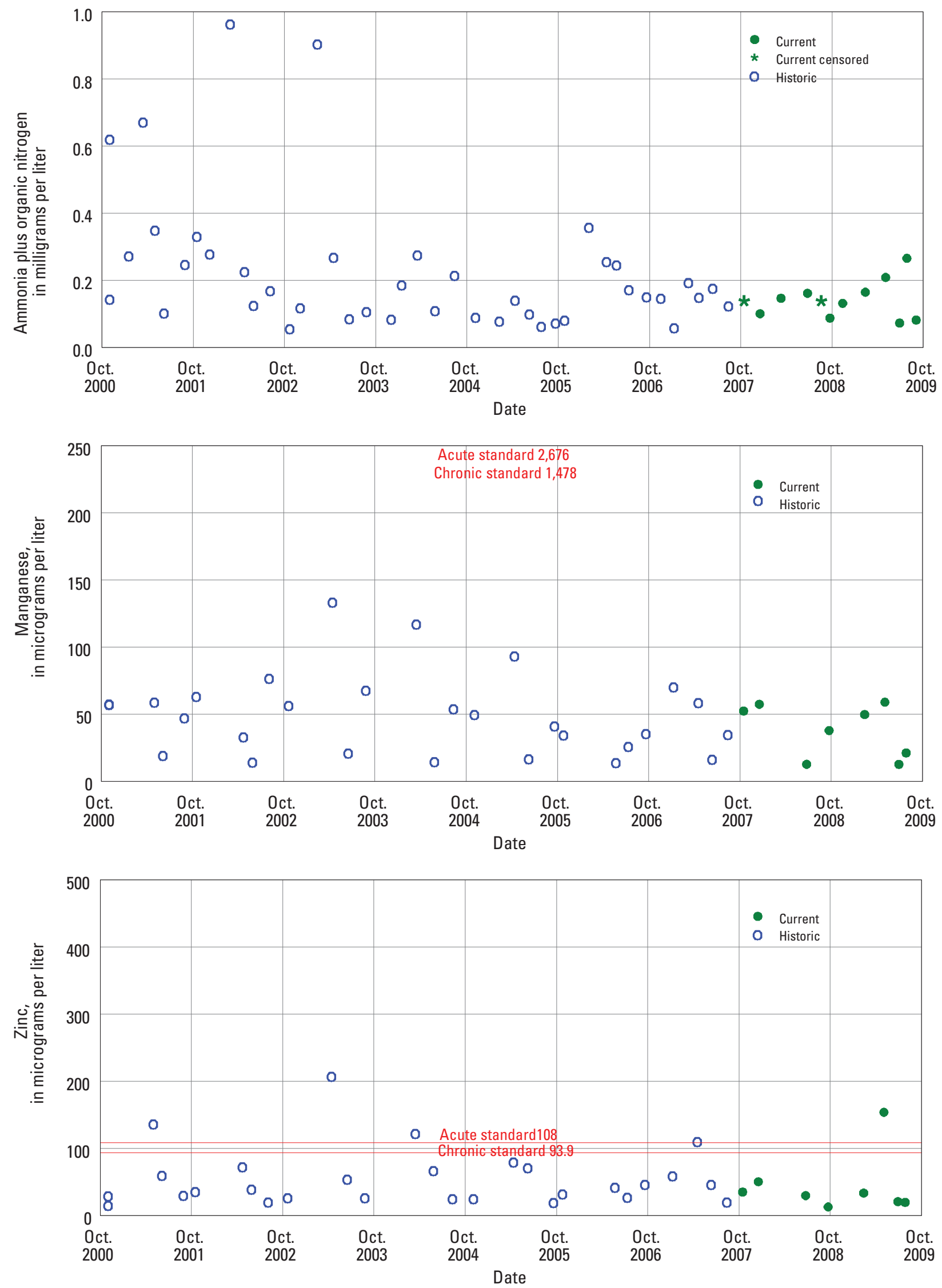

Figure 5. Time-series plots of selected water-quality constituents for Slate River near Crested Butte, Colorado. 
Table 4. Summary of measured constituents and properties for Slate River near Crested Butte, Colorado, station 09111500.

[--, no data or not applicable; L, low; M, medium; H, high; NTRU, Nephelometric Turbidity Ratio Unit; E. coli, Escherichia coli; mg/m² milligrams per square meter; USEPA, U.S. Environmental Protection Agency; CDPHE, Colorado Department of Public Health and Environment; Cd(ch), Cadmium, chronic standard; Zn(ch), Zinc, chronic standard]

\begin{tabular}{|c|c|c|c|c|c|c|c|c|c|c|c|c|c|c|}
\hline $\begin{array}{c}\text { Property } \\
\text { or } \\
\text { constituent }\end{array}$ & $\begin{array}{l}\text { Period } \\
\text { (water } \\
\text { years) }\end{array}$ & $\begin{array}{c}\text { Number } \\
\text { of } \\
\text { samples }\end{array}$ & $\begin{array}{c}\text { Number } \\
\text { of } \\
\text { censored } \\
\text { values }\end{array}$ & Median' & Maximum & $\begin{array}{c}\text { Date } \\
\text { of } \\
\text { Maximum }\end{array}$ & $\begin{array}{c}\text { 15th } \\
\text { percentile }\end{array}$ & $\begin{array}{c}\text { 85th } \\
\text { percentile }\end{array}$ & $\begin{array}{l}\text { Geometric } \\
\text { Mean }^{2}\end{array}$ & $\begin{array}{c}\text { Standard } \\
\text { or } \\
\text { Chronic } \\
\text { standard }^{3}\end{array}$ & $\begin{array}{l}\text { Number of } \\
\text { exceedences } \\
\text { Standard or } \\
\text { Chronic }\end{array}$ & $\begin{array}{c}\text { Acute } \\
\text { standard }\end{array}$ & $\begin{array}{c}\begin{array}{c}\text { Number } \\
\text { of } \\
\text { exceedences } \\
\text { Acute }\end{array} \\
\end{array}$ & $\begin{array}{c}\text { Level } \\
\text { of } \\
\text { concern }\end{array}$ \\
\hline $\begin{array}{l}\text { Instantaneous discharge, in cubic } \\
\text { feet per second }\end{array}$ & 1995-2007 & 83 & 0 & 32 & 1,210 & $06 / 05 / 97$ & 16 & 403 & -- & -- & -- & -- & -- & -- \\
\hline $\begin{array}{l}\text { Instantaneous discharge, in cubic } \\
\text { feet per second }\end{array}$ & 2008-2009 & 12 & 0 & 23 & 778 & $06 / 25 / 08$ & 10 & 402 & -- & -- & -- & -- & -- & -- \\
\hline Turbidity, in NTRU & 2001-2007 & 27 & 6 & 2.5 & 76 & $11 / 02 / 00$ & 0.0 & 11 & -- & -- & -- & -- & -- & -- \\
\hline Turbidity, in NTRU & 2008-2009 & 8 & 1 & 1.4 & 25 & $06 / 25 / 08$ & 0.4 & 19 & -- & -- & -- & -- & -- & -- \\
\hline $\begin{array}{l}\text { Dissolved oxygen, in milligrams } \\
\text { per liter }\end{array}$ & 1995-2007 & 80 & 0 & 9.0 & 10.6 & $11 / 19 / 96$ & 7.5 & 9.9 & -- & 6.0 & 0 & -- & -- & $\mathrm{L}$ \\
\hline $\begin{array}{l}\text { Dissolved oxygen, in milligrams } \\
\text { per liter }\end{array}$ & 2008-2009 & 12 & 0 & 8.9 & 10.1 & $12 / 18 / 07$ & 7.9 & 10.1 & -- & 6.0 & 0 & -- & -- & $\mathrm{L}$ \\
\hline $\mathrm{pH}$, in standard units & 1995-2007 & 80 & 0 & 7.6 & 8.3 & $05 / 20 / 96$ & 7.3 & 7.9 & -- & 6.5 & 0 & 9.0 & -- & $\mathrm{L}$ \\
\hline $\mathrm{pH}$, in standard units & 2008-2009 & 12 & 0 & 7.6 & 8.2 & $11 / 13 / 08$ & 7.2 & 8.2 & -- & 6.5 & 0 & 9.0 & -- & $\mathrm{L}$ \\
\hline $\begin{array}{l}\text { Specific conductance, in micro- } \\
\text { siemens per centimeter }\end{array}$ & 1995-2007 & 80 & 0 & 160 & 229 & $02 / 27 / 02$ & 81.1 & 204 & -- & -- & -- & -- & -- & -- \\
\hline $\begin{array}{l}\text { Specific conductance, in micro- } \\
\text { siemens per centimeter }\end{array}$ & 2008-2009 & 12 & 0 & 160 & 240 & $03 / 11 / 08$ & 91.0 & 206 & -- & -- & -- & -- & -- & -- \\
\hline Temperature, degrees Celsius & $1995-2007$ & 80 & 0 & 4.5 & 18.0 & $08 / 05 / 02$ & 0.1 & 11.7 & -- & 17.0 & 1 & -- & -- & $\mathrm{L}$ \\
\hline Temperature, degrees Celsius & 2008-2009 & 12 & 0 & 7.3 & 15.7 & 06/29/09 & 0.0 & 14.8 & -- & 17.0 & 0 & -- & -- & $\mathrm{L}$ \\
\hline Calcium, in milligrams per liter & 1995-2007 & 50 & 0 & 21.8 & 28.6 & ${ }^{4} 04 / 12 / 05$ & 10.5 & 26.0 & -- & -- & -- & -- & -- & -- \\
\hline Calcium, in milligrams per liter & 2008-2009 & 8 & 0 & 21.1 & 27.6 & $12 / 18 / 07$ & 10.9 & 26.9 & -- & -- & -- & -- & -- & -- \\
\hline $\begin{array}{l}\text { Magnesium, in milligrams per } \\
\text { liter }\end{array}$ & 1995-2007 & 50 & 0 & 2.68 & 4.74 & $04 / 12 / 05$ & 1.39 & 3.66 & -- & -- & -- & -- & -- & -- \\
\hline $\begin{array}{l}\text { Magnesium, in milligrams per } \\
\quad \text { liter }\end{array}$ & 2008-2009 & 8 & 0 & 2.64 & 3.80 & 02/12/09 & 1.22 & 3.76 & -- & -- & -- & -- & -- & -- \\
\hline $\begin{array}{l}\text { Ammonia plus organic nitrogen, } \\
\text { in milligrams per liter }\end{array}$ & 1995-2007 & 80 & 18 & 0.15 & 0.96 & $02 / 27 / 02$ & 5 & 0.33 & -- & -- & -- & - & - & -- \\
\hline $\begin{array}{l}\text { Ammonia plus organic nitrogen, } \\
\text { in milligrams per liter }\end{array}$ & 2008-2009 & 12 & 2 & 0.12 & 0.27 & 07/28/09 & 5 & 0.21 & -- & -- & -- & -- & -- & -- \\
\hline $\begin{array}{l}\text { Ammonia nitrogen, in mil- } \\
\text { ligrams per liter }\end{array}$ & 1995-2007 & 80 & 15 & 0.045 & 0.779 & $02 / 27 / 02$ & 5 & 0.180 & -- & 3.95 & 0 & 12.0 & 0 & $\mathrm{~L}$ \\
\hline $\begin{array}{l}\text { Ammonia nitrogen, in mil- } \\
\text { ligrams per liter }\end{array}$ & 2008-2009 & 12 & 6 & 0.008 & 0.130 & $07 / 28 / 09$ & 5 & 0.090 & -- & 3.84 & 0 & 12.2 & 0 & $\mathrm{~L}$ \\
\hline $\begin{array}{l}\text { Nitrite plus nitrate nitrogen, in } \\
\text { milligrams per liter }\end{array}$ & 1995-2007 & 80 & 0 & 0.168 & 0.710 & $02 / 18 / 97$ & 0.070 & 0.345 & -- & 10.0 & 0 & -- & -- & $\mathrm{L}$ \\
\hline $\begin{array}{l}\text { Nitrite plus nitrate nitrogen, in } \\
\text { milligrams per liter }\end{array}$ & 2008-2009 & 12 & 0 & 0.103 & 0.561 & $03 / 11 / 08$ & 0.047 & 0.353 & -- & 10.0 & 0 & -- & -- & $\mathrm{L}$ \\
\hline $\begin{array}{l}\text { Nitrite nitrogen, in milligrams } \\
\text { per liter }\end{array}$ & 1995-2007 & 80 & 29 & 0.002 & 0.030 & ${ }^{4} 10 / 24 / 95$ & 5 & 0.010 & -- & 0.05 & 0 & -- & -- & $\mathrm{L}$ \\
\hline
\end{tabular}


[--, no data or not applicable; L, low; M, medium; H, high; NTRU, Nephelometric Turbidity Ratio Unit; E. coli, Escherichia coli; mg/m² , milligrams per square meter; USEPA, U.S. Environmental Protection Agency; CDPHE, Colorado Department of Public Health and Environment; Cd(ch), Cadmium, chronic standard; Zn(ch), Zinc, chronic standard]

\begin{tabular}{|c|c|c|c|c|c|c|c|c|c|c|c|c|c|c|}
\hline $\begin{array}{l}\text { Property } \\
\text { or } \\
\text { constituent }\end{array}$ & $\begin{array}{l}\text { Period } \\
\text { (water } \\
\text { years) }\end{array}$ & $\begin{array}{l}\text { Number } \\
\text { of } \\
\text { samples }\end{array}$ & $\begin{array}{c}\text { Number } \\
\text { of } \\
\text { censored } \\
\text { values }\end{array}$ & Median' & Maximum & $\begin{array}{c}\text { Date } \\
\text { of } \\
\text { Maximum }\end{array}$ & $\begin{array}{c}\text { 15th } \\
\text { percentile }\end{array}$ & $\begin{array}{c}\text { 85th } \\
\text { percentile }\end{array}$ & $\begin{array}{l}\text { Geometric } \\
\text { Mean }^{2}\end{array}$ & $\begin{array}{c}\text { Standard } \\
\text { or } \\
\text { Chronic } \\
\text { standard }^{3}\end{array}$ & $\begin{array}{l}\text { Number of } \\
\text { exceedences } \\
\text { Standard or } \\
\text { Chronic }\end{array}$ & $\begin{array}{l}\text { Acute } \\
\text { standard }\end{array}$ & $\begin{array}{c}\begin{array}{c}\text { Number } \\
\text { of }\end{array} \\
\text { exceedences } \\
\text { Acute }\end{array}$ & $\begin{array}{l}\text { Level } \\
\text { of } \\
\text { concern }\end{array}$ \\
\hline $\begin{array}{l}\text { Nitrite nitrogen, in milligrams } \\
\text { per liter }\end{array}$ & 2008-2009 & 12 & 2 & 0.002 & 0.006 & 03/11/08 & 5 & 0.005 & -- & 0.05 & 0 & -- & -- & $\mathrm{L}$ \\
\hline $\begin{array}{l}\text { Orthophosphate, in milligrams } \\
\text { per liter }\end{array}$ & $1995-2007$ & 80 & 17 & 0.023 & 0.162 & $02 / 11 / 03$ & 5 & 0.071 & -- & -- & -- & -- & -- & -- \\
\hline $\begin{array}{l}\text { Orthophosphate, in milligrams } \\
\text { per liter }\end{array}$ & $2008-2009$ & 12 & 2 & 0.045 & 0.160 & 09/03/09 & 5 & 0.105 & -- & -- & -- & -- & -- & -- \\
\hline $\begin{array}{l}\text { Phosphorus (total), in milligrams } \\
\text { per liter }\end{array}$ & 1995-2007 & 79 & 8 & 0.040 & 0.206 & $02 / 11 / 03$ & 0.015 & 0.100 & -- & -- & -- & -- & -- & -- \\
\hline $\begin{array}{l}\text { Phosphorus (total), in milligrams } \\
\text { per liter }\end{array}$ & 2008-2009 & 12 & 0 & 0.062 & 0.174 & 09/03/09 & 0.027 & 0.133 & -- & -- & -- & -- & -- & -- \\
\hline $\begin{array}{l}\text { E. coli, in colonies per } 100 \\
\text { milliliter }\end{array}$ & $2001-2007$ & 41 & 7 & 6 & 220 & 08/14/07 & 1 & 6 & 6.0 & 126 & 3 & -- & -- & L \\
\hline $\begin{array}{l}\text { E. coli, in colonies per } 100 \\
\text { milliliter }\end{array}$ & $2008-2009$ & 12 & 2 & 6 & 50 & 07/28/09 & 1 & 6 & -- & 126 & 0 & -- & -- & $\mathrm{L}$ \\
\hline $\begin{array}{l}\text { Biomass periphyton, ashfree } \\
\text { drymass, in grams per square } \\
\text { meter }\end{array}$ & $1997-2007$ & 8 & 2 & 9.4 & 22.2 & 08/14/07 & 5 & 20.9 & -- & -- & -- & -- & -- & -- \\
\hline $\begin{array}{l}\text { Biomass periphyton, ashfree } \\
\text { drymass, in grams per square } \\
\text { meter }\end{array}$ & $2008-2009$ & 2 & 0 & 18.0 & 26.9 & 09/03/09 & 9.1 & 26.9 & -- & -- & -- & -- & -- & -- \\
\hline $\begin{array}{l}\text { Periphyton, biomass, ash weight, } \\
\text { in grams per square meter }\end{array}$ & $1997-2007$ & 8 & 0 & 489 & 842 & $08 / 26 / 03$ & 71.6 & 779 & -- & -- & -- & -- & -- & -- \\
\hline $\begin{array}{l}\text { Periphyton, biomass, ash weight, } \\
\text { in grams per square meter }\end{array}$ & $2008-2009$ & 2 & 0 & 498 & 792 & 09/03/09 & 205 & 792 & -- & -- & -- & -- & -- & -- \\
\hline $\begin{array}{l}\text { Periphyton, biomass, dry weight, } \\
\text { in grams per square meter }\end{array}$ & $1997-2007$ & 8 & 0 & 509 & 858 & $08 / 26 / 03$ & 76.2 & 795 & -- & -- & -- & -- & -- & -- \\
\hline $\begin{array}{l}\text { Periphyton, biomass, dry weight, } \\
\text { in grams per square meter }\end{array}$ & $2008-2009$ & 2 & 0 & 516 & 819 & 09/03/09 & 214 & 819 & -- & -- & -- & -- & -- & -- \\
\hline $\begin{array}{l}\text { Chlorophyll a, periphyton, } \\
\text { chromofluoro, in } \mathrm{mg} / \mathrm{m}^{2}\end{array}$ & $1997-2007$ & 8 & 0 & 14.6 & 84.7 & 08/14/07 & 2.05 & 66.4 & -- & -- & -- & -- & -- & -- \\
\hline $\begin{array}{l}\text { Chlorophyll a, periphyton, } \\
\text { chromofluoro, in } \mathrm{mg} / \mathrm{m}^{2}\end{array}$ & $2008-2009$ & 2 & 0 & 31.2 & 35.1 & 09/03/09 & 27.2 & 35.1 & -- & -- & -- & -- & -- & -- \\
\hline $\begin{array}{l}\text { Pheophytin a, periphyton, in } \\
\mathrm{mg} / \mathrm{m}^{2}\end{array}$ & $2002-2007$ & 6 & 0 & 5.9 & 30.7 & 08/14/07 & 0.8 & 30.1 & -- & -- & -- & -- & -- & -- \\
\hline $\begin{array}{l}\text { Pheophytin a, periphyton, in } \\
\mathrm{mg} / \mathrm{m}^{2}\end{array}$ & 2008-2009 & 2 & 0 & 15.2 & 22.2 & 09/03/09 & 8.3 & 22.2 & -- & -- & -- & -- & -- & -- \\
\hline $\begin{array}{l}\text { Aluminum, in micrograms per } \\
\text { liter }\end{array}$ & 1999-2007 & 36 & 2 & 12.7 & 65.2 & $05 / 02 / 01$ & 5.3 & 26.8 & -- & -- & -- & -- & -- & -- \\
\hline $\begin{array}{l}\text { Aluminum, in micrograms per } \\
\text { liter }\end{array}$ & 2008-2009 & 8 & 0 & 10.0 & 74.6 & 05/04/09 & 5.2 & 61.0 & -- & -- & -- & -- & -- & -- \\
\hline
\end{tabular}


Table 4. Summary of measured constituents and properties for Slate River near Crested Butte, Colorado, station 09111500.—Continued

[--, no data or not applicable; L, low; M, medium; H, high; NTRU, Nephelometric Turbidity Ratio Unit; E. coli, Escherichia coli; mg/m² , milligrams per square meter; USEPA, U.S. Environmental Protection Agency; CDPHE, Colorado Department of Public Health and Environment; Cd(ch), Cadmium, chronic standard; Zn(ch), Zinc, chronic standard]

\begin{tabular}{|c|c|c|c|c|c|c|c|c|c|c|c|c|c|c|}
\hline $\begin{array}{c}\text { Property } \\
\text { or } \\
\text { constituent }\end{array}$ & $\begin{array}{l}\text { Period } \\
\text { (water } \\
\text { years) }\end{array}$ & $\begin{array}{c}\text { Number } \\
\text { of } \\
\text { samples }\end{array}$ & $\begin{array}{l}\text { Number } \\
\text { of } \\
\text { censored } \\
\text { values }\end{array}$ & Median' & Maximum & $\begin{array}{c}\text { Date } \\
\text { of } \\
\text { Maximum }\end{array}$ & $\begin{array}{c}\text { 15th } \\
\text { percentile }\end{array}$ & $\begin{array}{c}\text { 85th } \\
\text { percentile }\end{array}$ & $\begin{array}{c}\text { Geometric } \\
\text { Mean }^{2}\end{array}$ & $\begin{array}{c}\text { Standard } \\
\text { or } \\
\text { Chronic } \\
\text { standard }^{3}\end{array}$ & $\begin{array}{l}\text { Number of } \\
\text { exceedences } \\
\text { Standard or } \\
\text { Chronic }\end{array}$ & $\begin{array}{l}\text { Acute } \\
\text { standard }\end{array}$ & $\begin{array}{c}\text { Number } \\
\text { of } \\
\text { exceedences } \\
\text { Acute }\end{array}$ & $\begin{array}{c}\text { Level } \\
\text { of } \\
\text { concern }\end{array}$ \\
\hline $\begin{array}{l}\text { Cadmium, in micrograms per } \\
\text { liter }\end{array}$ & 1999-2007 & 36 & 6 & 0.21 & 1.19 & $04 / 14 / 99$ & 5 & 0.42 & -- & ${ }^{8} 0.33$ & 10 & 1.28 & 0 & $\mathrm{H}$ \\
\hline $\begin{array}{l}\text { Cadmium, in micrograms per } \\
\text { liter }\end{array}$ & 2008-2009 & 8 & 0 & 0.21 & 0.93 & 05/04/09 & 0.15 & 0.70 & -- & ${ }^{8} 0.33$ & 1 & 1.28 & 0 & $\mathrm{H}$ \\
\hline Copper, in micrograms per liter & 1999-2007 & 36 & 8 & 1.0 & 6.6 & $06 / 12 / 07$ & 5 & 2.6 & -- & 6.8 & 0 & 9.9 & 0 & $\mathrm{~L}$ \\
\hline Copper, in micrograms per liter & $2008-2009$ & 8 & 0 & 1.0 & 8.1 & $10 / 16 / 07$ & 0.60 & 6.4 & -- & 6.8 & 1 & 9.9 & 0 & M \\
\hline Iron, in micrograms per liter & $1995-2007$ & 43 & 0 & 45 & 85 & $11 / 19 / 98$ & 25 & 71 & -- & 300 & 0 & -- & -- & $\mathrm{L}$ \\
\hline Iron, in micrograms per liter & 2008-2009 & 8 & 0 & 71 & 106 & $12 / 18 / 07$ & 38 & 105 & -- & 300 & 0 & -- & -- & $\mathrm{L}$ \\
\hline Lead, in micrograms per liter & 1999-2007 & 36 & 17 & 0.07 & 1.04 & $06 / 17 / 03$ & 5 & 0.67 & -- & 1.76 & 0 & 45.1 & 0 & $\mathrm{~L}$ \\
\hline Lead, in micrograms per liter & $2008-2009$ & 8 & 0 & 0.39 & 1.17 & $10 / 16 / 07$ & 0.08 & 1.05 & -- & 1.76 & 0 & 45.1 & 0 & M \\
\hline $\begin{array}{l}\text { Manganese, in micrograms per } \\
\text { liter }\end{array}$ & $1995-2007$ & 43 & 0 & 47.0 & 158 & $04 / 14 / 99$ & 15.8 & 83.2 & -- & 1,478 & 0 & 2,676 & 0 & $\mathrm{~L}$ \\
\hline $\begin{array}{l}\text { Manganese, in micrograms per } \\
\text { liter }\end{array}$ & 2008-2009 & 8 & 0 & 43.9 & 59.0 & 05/04/09 & 12.8 & 58.5 & -- & 1,478 & 0 & 2,676 & 0 & $\mathrm{~L}$ \\
\hline Silver, in micrograms per liter & 1999-2007 & 36 & 36 & 5 & ${ }^{7}<0.3$ & -- & 5 & 5 & -- & 0.04 & 0 & 1.15 & 0 & $\mathrm{~L}$ \\
\hline Silver, in micrograms per liter & 2008-2009 & 8 & 7 & 5 & ${ }^{7}<0.1$ & -- & 5 & 5 & -- & 0.04 & 0 & 1.15 & 0 & $\mathrm{~L}$ \\
\hline Zinc, in micrograms per liter & 1999-2007 & 36 & 0 & 40.6 & 455 & $04 / 14 / 99$ & 22.4 & 93.0 & -- & 93.9 & 5 & 108 & 5 & M \\
\hline Zinc, in micrograms per liter & 2008-2009 & 8 & 0 & 31.8 & 154 & 05/04/09 & 15.3 & 118 & -- & 93.9 & 1 & 108 & 1 & $\mathrm{H}$ \\
\hline $\begin{array}{l}\text { Suspended sediment, in mil- } \\
\text { ligrams per liter }\end{array}$ & $1999-2007$ & 35 & 0 & 4.7 & 234 & $11 / 02 / 00$ & 1.0 & 28.0 & -- & -- & -- & -- & -- & -- \\
\hline $\begin{array}{l}\text { Suspended sediment, in mil- } \\
\text { ligrams per liter }\end{array}$ & 2008-2009 & 7 & 0 & 2.0 & 46.0 & $06 / 25 / 08$ & 1.0 & 39.8 & -- & -- & -- & -- & -- & -- \\
\hline
\end{tabular}

${ }^{1}$ Censored values were replaced with 0 to compute median and 85 th percentiles (coliform censored values replaced with 1) see definitions section.

${ }^{2}$ Geometric mean listed for E. coli. Geometric mean computed for combined period of record (2001-2009).

${ }^{3}$ Colorado Department of Public Health and Environment, classification and numeric standards for Gunnison and Lower Dolores River Basins (2010a); and USEPA National Recommended Water Quality Criteria (2009).

${ }^{4}$ Multiple dates for maximum.

${ }^{5}$ Median values and percentiles were not tabled when they were calculated as zero.

${ }^{6}$ Use geometric mean for comparison to standard.

${ }^{7}$ All values below laboratory reporting level (LRL), current LRL used for current period maximum, highest LRL used for historic period.

${ }^{8}$ Temporary modifications to CDPHE numeric standards for this stream segment: $\mathrm{Cd}(\mathrm{ch})=0.4$ Expiration Date of 12/31/2012. 
Table 5. Summary of seasonal Kendall trend tests for Slate River near Crested Butte, Colorado, station 09111500.

[E.coli, Escherichia coli]

\begin{tabular}{|c|c|c|c|c|}
\hline Property or constituent & $\begin{array}{c}\text { Period } \\
\text { (water years) }\end{array}$ & Kendall tau $(\tau)$ & p-Value & Trend direction \\
\hline Specific conductance, in microsiemens per centimeter & 1995-2009 & 0.195 & 0.048 & Up \\
\hline $\mathrm{pH}$, in standard units & 1995-2009 & 0.020 & 0.859 & None \\
\hline Ammonia nitrogen, in milligrams per liter & $2000-2009$ & -0.167 & 0.179 & None \\
\hline Nitrite nitrogen, in milligrams per liter & $2000-2009$ & -0.144 & 0.246 & None \\
\hline Ammonia plus organic nitrogen, in milligrams per liter & 1999-2009 & -0.291 & 0.014 & Down \\
\hline Nitrite plus nitrate nitrogen, in milligrams per liter & $1995-2009$ & -0.071 & 0.478 & None \\
\hline Phosphorus (total), in milligrams per liter & 2000-2009 & 0.122 & 0.348 & None \\
\hline Orthophosphate, in milligrams per liter & $2000-2009$ & 0.233 & 0.062 & None \\
\hline Calcium, in milligrams per liter & 1999-2009 & -0.051 & 0.759 & None \\
\hline Magnesium, in milligrams per liter & 1999-2009 & -0.124 & 0.414 & None \\
\hline Cadmium, in micrograms per liter & $2001-2009$ & -0.044 & 0.838 & None \\
\hline Copper, in micrograms per liter & 2000-2009 & 0.296 & 0.063 & None \\
\hline Iron, in micrograms per liter & 1999-2009 & 0.022 & 0.919 & None \\
\hline Manganese, in micrograms per liter & 1999-2009 & -0.416 & 0.004 & Down \\
\hline Zinc, in micrograms per liter & 1999-2009 & -0.197 & 0.184 & None \\
\hline Suspended sediment, in milligrams per liter & 1999-2009 & 0.051 & 0.757 & None \\
\hline E. coli, in colonies per 100 milliliter & 2001-2009 & -0.264 & 0.053 & None \\
\hline
\end{tabular}




\section{Slate River above Baxter Gulch near Crested Butte, Colorado}

Current Reason for Inclusion: This station characterizes water quality downstream from Crested Butte.

General Station Information:

Location:

Station Type:

Period of Record:

Streamflow gaging:

Latitude: 385106

Longitude: 1065710
$80-400 \mathrm{ft}$ downstream from State Highway 135 bridge (2.0 mi southeast of Crested Butte, and 5.0 mi upstream from mouth.)

USGS water-quality and streamflow gaging

Water quality: January 2007-September 2009

October 2006-September 2009

Drainage area: Not determined

Stream segment: 8
HUC: 14020002

USGS Data Summary: Water Years 2008-2009:

General Chemistry:

pH: Low concern

Dissolved oxygen: Low concern

Temperature: Low concern

Nutrients:

Low concern

E. coli:

Low concern

Trace Elements/metals:

No data

Other constituents of concern: None for constituents tested.

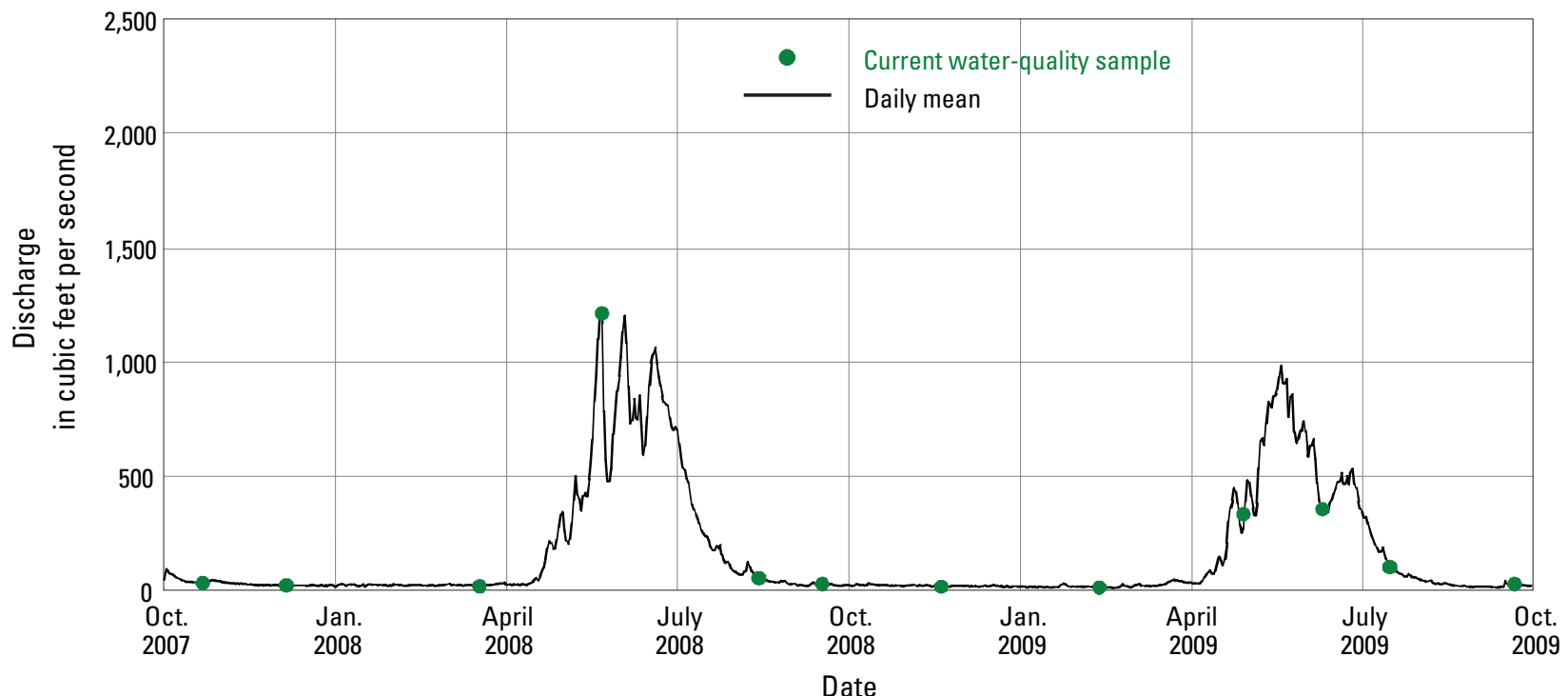

Figure 6. Daily mean discharge and time distribution of water-quality samples for Slate River above Baxter Gulch, Colorado. 

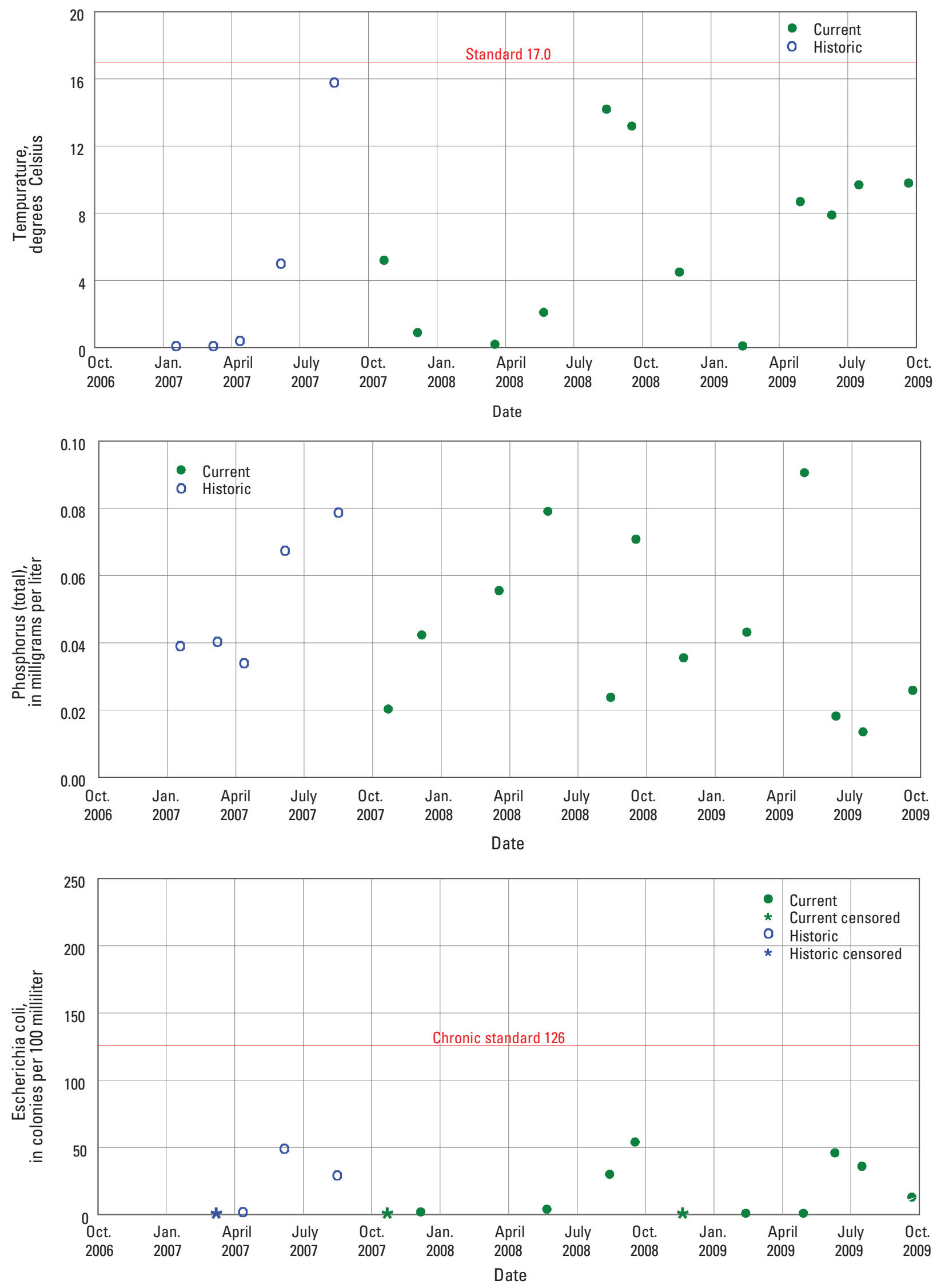

Figure 7. Time-series plots of selected water-quality constituents for Slate River above Baxter Gulch, Colorado. 
Table 6. Summary of measured constituents and properties for Slate River above Baxter Gulch near Crested Butte, Colorado, station 385106106571000.

[--, no data or not applicable; L, low; M, medium; H, high; E. coli, Escherichia coli; mg/m², milligrams per square meter; USEPA, U.S. Environmental Protection Agency]

\begin{tabular}{|c|c|c|c|c|c|c|c|c|c|c|c|c|c|c|}
\hline $\begin{array}{l}\text { Property } \\
\text { or } \\
\text { constituent }\end{array}$ & $\begin{array}{l}\text { Period } \\
\text { (water } \\
\text { years) }\end{array}$ & $\begin{array}{l}\text { Number } \\
\text { of } \\
\text { samples }\end{array}$ & $\begin{array}{l}\text { Number } \\
\quad \text { of } \\
\text { censored } \\
\text { values }\end{array}$ & Median' & Maximum & $\begin{array}{c}\text { Date } \\
\text { of } \\
\text { Maximum }\end{array}$ & $\begin{array}{c}\text { 15th } \\
\text { percentile }\end{array}$ & $\begin{array}{c}\text { 85th } \\
\text { percentile }\end{array}$ & $\begin{array}{c}\text { Geometric } \\
\text { Mean }^{2}\end{array}$ & $\begin{array}{l}\text { Standard } \\
\text { or } \\
\text { Chronic } \\
\text { standard }^{3}\end{array}$ & $\begin{array}{l}\text { Number of } \\
\text { exceedences } \\
\text { Standard or } \\
\text { Chronic }\end{array}$ & $\begin{array}{l}\text { Acute } \\
\text { standard }\end{array}$ & $\begin{array}{c}\begin{array}{c}\text { Number } \\
\text { of }\end{array} \\
\text { exceedences } \\
\text { Acute }\end{array}$ & $\begin{array}{c}\text { Level } \\
\text { of } \\
\text { concern }\end{array}$ \\
\hline $\begin{array}{l}\text { Instantaneous discharge, in } \\
\text { cubic feet per second }\end{array}$ & 1995-2007 & 7 & 0 & 28.0 & 434 & $06 / 06 / 07$ & 20.7 & 374 & -- & -- & -- & -- & -- & -- \\
\hline $\begin{array}{l}\text { Instantaneous discharge, in } \\
\text { cubic feet per second }\end{array}$ & 2008-2009 & 12 & 0 & 28.0 & 1210 & $05 / 22 / 08$ & 14.2 & 397 & -- & -- & -- & -- & -- & -- \\
\hline $\begin{array}{l}\text { Dissolved oxygen, in milligrams } \\
\text { per liter }\end{array}$ & 1995-2007 & 7 & 0 & 9.0 & 10.0 & ${ }^{4} 03 / 25 / 95$ & 8.3 & 10.0 & -- & 6.0 & 0 & -- & -- & $\mathrm{L}$ \\
\hline $\begin{array}{l}\text { Dissolved oxygen, in milligrams } \\
\text { per liter }\end{array}$ & 2008-2009 & 12 & 0 & 9.0 & 10.4 & $11 / 20 / 08$ & 7.7 & 10.3 & -- & 6.0 & 0 & -- & -- & $\mathrm{L}$ \\
\hline $\mathrm{pH}$, in standard units & 1995-2007 & 7 & 0 & 7.6 & 8.1 & $03 / 25 / 95$ & 7.5 & 8.0 & -- & 6.5 & 0 & 9.0 & -- & $\mathrm{L}$ \\
\hline $\mathrm{pH}$, in standard units & 2008-2009 & 12 & 0 & 7.6 & 8.3 & $10 / 22 / 07$ & 7.2 & 8.1 & -- & 6.5 & 0 & 9.0 & -- & L \\
\hline $\begin{array}{l}\text { Specific conductance, in micro- } \\
\text { siemens per centimeter }\end{array}$ & 1995-2007 & 7 & 0 & 195 & 231 & 03/08/07 & 96.8 & 227 & -- & -- & -- & -- & -- & -- \\
\hline $\begin{array}{l}\text { Specific conductance, in micro- } \\
\text { siemens per centimeter }\end{array}$ & 2008-2009 & 12 & 0 & 184 & 241 & 03/18/08 & 90.6 & 227 & -- & -- & -- & -- & -- & -- \\
\hline Temperature, degrees Celsius & $1995-2007$ & 7 & 0 & 0.4 & 15.8 & $08 / 16 / 07$ & 0.0 & 13.6 & -- & 17.0 & 0 & -- & -- & $\mathrm{L}$ \\
\hline Temperature, degrees Celsius & 2008-2009 & 12 & 0 & 6.6 & 14.2 & $08 / 14 / 08$ & 0.2 & 13.3 & -- & 17.0 & 0 & -- & -- & $\mathrm{L}$ \\
\hline Calcium, in milligrams per liter & 1995-2007 & 7 & 0 & 26.0 & 31.0 & ${ }^{4} 03 / 25 / 95$ & 13.9 & 30.9 & -- & -- & -- & -- & -- & -- \\
\hline Calcium, in milligrams per liter & 2008-2009 & 12 & 0 & 25.9 & 32.7 & 03/18/08 & 13.2 & 30.0 & -- & -- & -- & -- & -- & -- \\
\hline $\begin{array}{l}\text { Magnesium, in milligrams } \\
\text { per liter }\end{array}$ & 1995-2007 & 7 & 0 & 3.70 & 4.50 & $03 / 25 / 95$ & 1.66 & 4.45 & -- & -- & -- & -- & -- & -- \\
\hline $\begin{array}{l}\text { Magnesium, in milligrams } \\
\text { per liter }\end{array}$ & 2008-2009 & 12 & 0 & 3.41 & 4.87 & 03/18/08 & 1.76 & 4.51 & -- & -- & -- & -- & -- & -- \\
\hline $\begin{array}{l}\text { Ammonia plus organic nitro- } \\
\text { gen, in milligrams per liter }\end{array}$ & 1995-2007 & 7 & 2 & 0.12 & 0.21 & $04 / 12 / 07$ & 5 & 0.20 & -- & -- & -- & -- & -- & -- \\
\hline $\begin{array}{l}\text { Ammonia plus organic nitro- } \\
\text { gen, in milligrams per liter }\end{array}$ & 2008-2009 & 12 & 0 & 0.11 & 0.39 & 04/29/09 & 0.08 & 0.28 & -- & -- & -- & -- & -- & -- \\
\hline $\begin{array}{l}\text { Ammonia nitrogen, in mil- } \\
\text { ligrams per liter }\end{array}$ & 1995-2007 & 7 & 3 & 0.024 & 0.130 & $12 / 28 / 96$ & 5 & 0.118 & -- & 3.57 & 0 & 10.1 & 0 & $\mathrm{~L}$ \\
\hline $\begin{array}{l}\text { Ammonia nitrogen, in mil- } \\
\text { ligrams per liter }\end{array}$ & 2008-2009 & 12 & 5 & 0.011 & 0.041 & 03/18/08 & 5 & 0.026 & -- & 4.01 & 0 & 12.8 & 0 & $\mathrm{~L}$ \\
\hline $\begin{array}{l}\text { Nitrite plus nitrate nitrogen, in } \\
\text { milligrams per liter }\end{array}$ & 1995-2007 & 7 & 0 & 0.249 & 0.390 & $03 / 25 / 95$ & 0.092 & 0.363 & -- & 10.0 & 0 & -- & -- & L \\
\hline $\begin{array}{l}\text { Nitrite plus nitrate nitrogen, in } \\
\text { milligrams per liter }\end{array}$ & 2008-2009 & 12 & 0 & 0.118 & 0.289 & 03/18/08 & 0.043 & 0.246 & -- & 10.0 & 0 & -- & -- & $\mathrm{L}$ \\
\hline $\begin{array}{l}\text { Nitrite nitrogen, in milligrams } \\
\text { per liter }\end{array}$ & 1995-2007 & 7 & 4 & 5 & 0.004 & $08 / 16 / 07$ & 5 & 0.004 & -- & 0.05 & 0 & -- & -- & $\mathrm{L}$ \\
\hline $\begin{array}{l}\text { Nitrite nitrogen, in milligrams } \\
\text { per liter }\end{array}$ & 2008-2009 & 12 & 5 & 0.001 & 0.005 & 03/18/08 & 5 & 0.003 & -- & 0.05 & 0 & -- & -- & $\mathrm{L}$ \\
\hline $\begin{array}{l}\text { Orthophosphate, in milligrams } \\
\text { per liter }\end{array}$ & 1995-2007 & 7 & 1 & 0.030 & 0.148 & 03/08/07 & 0.001 & 0.131 & -- & -- & -- & -- & -- & -- \\
\hline
\end{tabular}


Table 6. Summary of measured constituents and properties for Slate River above Baxter Gulch near Crested Butte, Colorado, station 385106106571000.-Continued

[--, no data or not applicable; L, low; M, medium; H, high; E. coli, Escherichia coli; mg $/ \mathrm{m}^{2}$, milligrams per square meter; USEPA, U.S. Environmental Protection Agency]

\begin{tabular}{|c|c|c|c|c|c|c|c|c|c|c|c|c|c|c|}
\hline $\begin{array}{l}\text { Property } \\
\text { or } \\
\text { constituent }\end{array}$ & $\begin{array}{l}\text { Period } \\
\text { (water } \\
\text { years) }\end{array}$ & $\begin{array}{l}\text { Number } \\
\text { of } \\
\text { samples }\end{array}$ & $\begin{array}{c}\text { Number } \\
\text { of } \\
\text { censored } \\
\text { values }\end{array}$ & Median' ${ }^{1}$ & Maximum & $\begin{array}{c}\text { Date } \\
\text { of } \\
\text { Maximum }\end{array}$ & $\begin{array}{c}\text { 15th } \\
\text { percentile }\end{array}$ & $\begin{array}{c}\text { 85th } \\
\text { percentile }{ }^{1}\end{array}$ & $\begin{array}{l}\text { Geometric } \\
\text { Mean }^{2}\end{array}$ & $\begin{array}{l}\text { Standard } \\
\text { or } \\
\text { Chronic } \\
\text { standard }^{3}\end{array}$ & $\begin{array}{l}\text { Number of } \\
\text { exceedences } \\
\text { Standard or } \\
\text { Chronic }\end{array}$ & $\begin{array}{l}\text { Acute } \\
\text { standard }\end{array}$ & 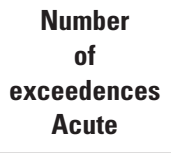 & $\begin{array}{l}\text { Level } \\
\text { of } \\
\text { concern }\end{array}$ \\
\hline $\begin{array}{l}\text { Orthophosphate, in milligrams } \\
\text { per liter }\end{array}$ & 2008-2009 & 12 & 1 & 0.012 & 0.047 & $09 / 17 / 08$ & 0.004 & 0.028 & -- & -- & -- & -- & -- & -- \\
\hline $\begin{array}{l}\text { Phosphorus (total), in mil- } \\
\text { ligrams per liter }\end{array}$ & 1995-2007 & 7 & 0 & 0.040 & 0.079 & 08/16/07 & 0.035 & 0.077 & -- & -- & -- & -- & -- & -- \\
\hline $\begin{array}{l}\text { Phosphorus (total), in mil- } \\
\text { ligrams per liter }\end{array}$ & 2008-2009 & 12 & 0 & 0.039 & 0.091 & 04/29/09 & 0.018 & 0.080 & -- & -- & -- & -- & -- & -- \\
\hline $\begin{array}{l}\text { E. coli, in colonies per } 100 \\
\text { milliliter }\end{array}$ & $2007-2007$ & 4 & 1 & 16 & 49 & 06/06/07 & 1 & 6 & 7.0 & 126 & 0 & -- & -- & $\mathrm{L}$ \\
\hline $\begin{array}{l}\text { E. coli, in colonies per } 100 \\
\text { milliliter }\end{array}$ & 2008-2009 & 11 & 2 & 4 & 54 & $09 / 17 / 08$ & 1 & 6 & -- & 126 & 0 & -- & -- & L \\
\hline $\begin{array}{l}\text { Biomass periphyton, ashfree } \\
\text { drymass, in grams per } \\
\text { square meter }\end{array}$ & $1997-2007$ & 2 & 0 & 3.2 & 5.9 & 08/16/07 & 0.5 & 5.9 & -- & -- & -- & -- & -- & -- \\
\hline $\begin{array}{l}\text { Biomass periphyton, ashfree } \\
\text { drymass, in grams per } \\
\text { square meter }\end{array}$ & 2008-2009 & 2 & 1 & 11.2 & 22.3 & $09 / 21 / 09$ & 5 & 22.3 & -- & -- & -- & -- & -- & -- \\
\hline $\begin{array}{l}\text { Periphyton, biomass, ash } \\
\text { weight, in grams per square } \\
\text { meter }\end{array}$ & 1997-2007 & 2 & 0 & 130 & 240 & 08/16/07 & 19.9 & 240 & -- & -- & -- & -- & -- & -- \\
\hline $\begin{array}{l}\text { Periphyton, biomass, ash } \\
\text { weight, in grams per square } \\
\text { meter }\end{array}$ & 2008-2009 & 2 & 0 & 398 & 508 & $09 / 21 / 09$ & 287 & 508 & -- & -- & -- & -- & -- & -- \\
\hline $\begin{array}{l}\text { Periphyton, biomass, dry } \\
\text { weight, in grams per square } \\
\text { meter }\end{array}$ & 1997-2007 & 2 & 0 & 133 & 246 & $08 / 16 / 07$ & 20.4 & 246 & -- & -- & -- & -- & -- & -- \\
\hline $\begin{array}{l}\text { Periphyton, biomass, dry } \\
\text { weight, in grams per square } \\
\text { meter }\end{array}$ & 2008-2009 & 2 & 0 & 412 & 531 & $09 / 21 / 09$ & 292 & 531 & -- & -- & -- & -- & -- & -- \\
\hline $\begin{array}{l}\text { Chlorophyll a, periphyton, } \\
\text { chromofluoro, in } \mathrm{mg} / \mathrm{m}^{2}\end{array}$ & $1997-2007$ & 2 & 0 & 3.80 & 7.40 & 08/16/07 & 0.20 & 7.40 & -- & -- & -- & -- & -- & -- \\
\hline $\begin{array}{l}\text { Chlorophyll a, periphyton, } \\
\text { chromofluoro, in } \mathrm{mg} / \mathrm{m}^{2}\end{array}$ & 2008-2009 & 2 & 0 & 25.0 & 31.2 & 09/21/09 & 18.9 & 31.2 & -- & -- & -- & -- & -- & -- \\
\hline $\begin{array}{l}\text { Pheophytin a, periphyton, in } \\
\mathrm{mg} / \mathrm{m}^{2}\end{array}$ & $2007-2007$ & 1 & 0 & -- & 2.2 & 08/16/07 & -- & -- & -- & -- & -- & -- & -- & -- \\
\hline $\begin{array}{l}\text { Pheophytin a, periphyton, in } \\
\mathrm{mg} / \mathrm{m}^{2}\end{array}$ & 2008-2009 & 2 & 0 & 10.5 & 12.6 & $09 / 21 / 09$ & 8.4 & 12.6 & -- & -- & -- & -- & -- & -- \\
\hline
\end{tabular}

${ }^{1}$ Censored values were replaced with 0 to compute median and 85 th percentiles (coliform censored values replaced with 1 ) see definitions section.

${ }^{2}$ Geometric mean listed for E. coli. Geometric mean computed for combined period of record (2007-2009).

${ }^{3}$ Colorado Department of Public Health and Environment, classification and numeric standards for Gunnison and Lower Dolores River Basins (2010a); and USEPA National Recommended Water Quality Criteria (2009).

${ }^{4}$ Multiple dates for maximum.

${ }^{5}$ Median values and percentiles were not tabled when they were calculated as zero.

${ }^{6}$ Use geometric mean for comparison to standard. 


\section{Slate River above Squaw Gulch near Crested Butte, Colorado}

Current Reason for Inclusion: This station is part of the rotational group of sites.

This station characterizes water quality directly downstream from the Riverland Industrial Park.

General Station Information:

Location:

5-50 ft upstream or 10-100 ft downstream from rural bridge. (3.0 mi southeast of Crested Butte, and 4.0 mi upstream from mouth)

Station Type:

USGS water-quality

Period of Record:

Water quality: January 2007-September 2009

Latitude: 385012

Drainage area: Not determined

HUC: 14020002

Longitude: 1065612

Stream segment: 8

USGS Data Summary: Water Years 2008-2009:

General Chemistry:

pH: Low concern

Dissolved oxygen: Low concern

Temperature: Low concern

Nutrients:

Low concern

E. coli:

Low concern

Trace Elements/metals:

High concern: Cadmium and zinc

Other constituents of concern: $\quad$ None for constituents tested.

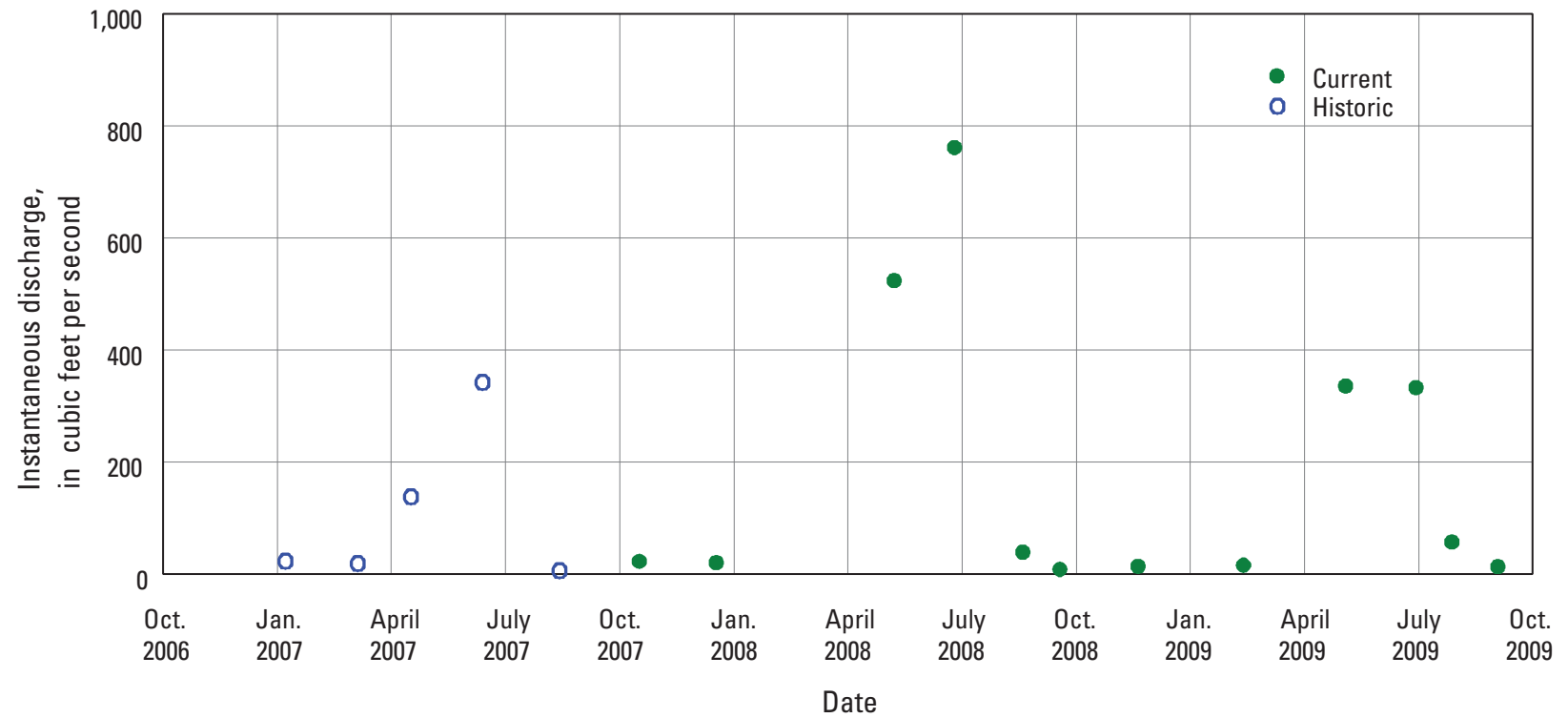

Figure 8. Time distribution and associated discharge of water-quality samples for Slate River above Squaw Gulch, Colorado. 

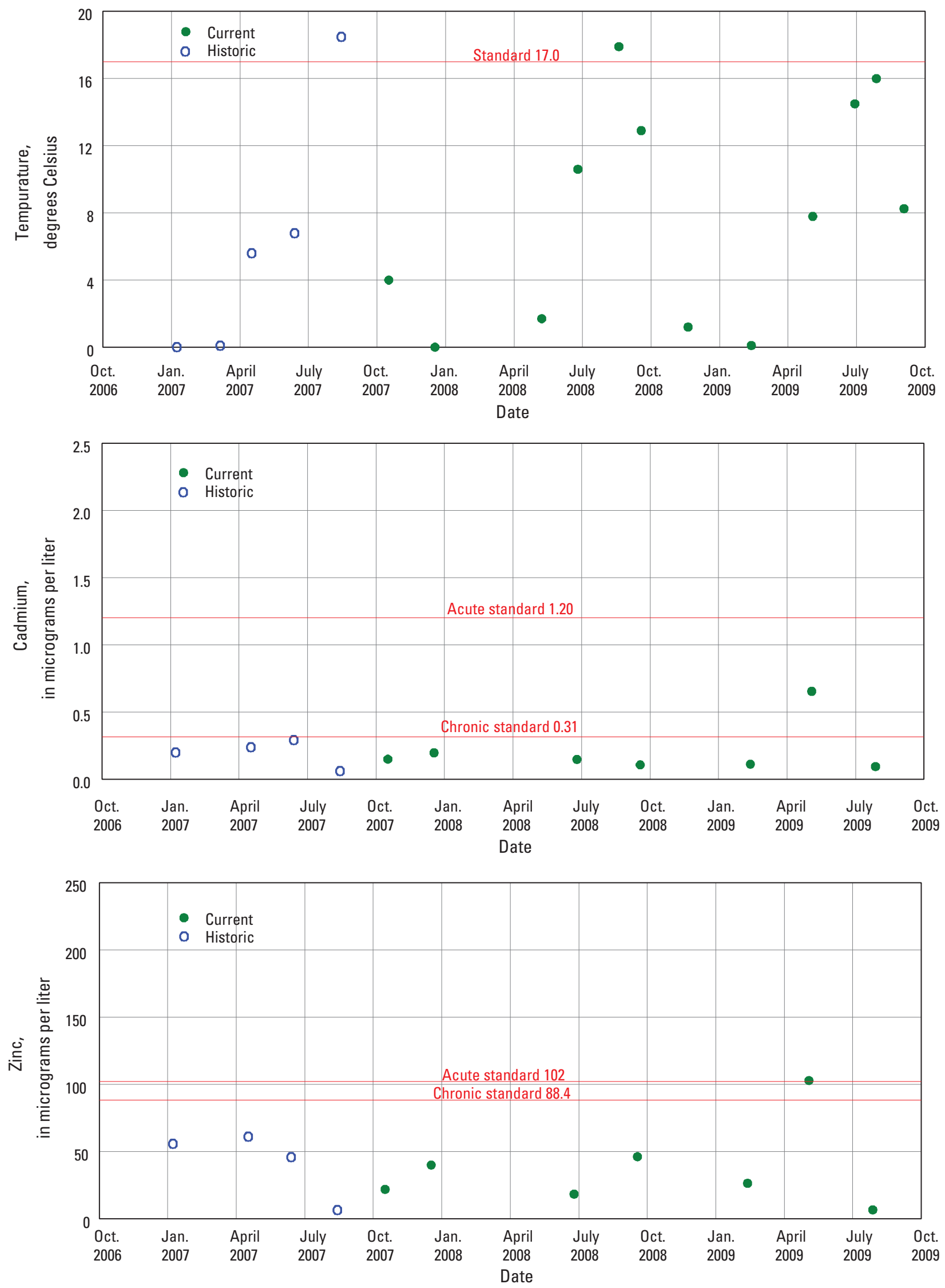

Figure 9. Time-series plots of selected water-quality constituents for Slate River above Squaw Gulch, Colorado. 
Table 7. Summary of measured constituents and properties for Slate River above Squaw Gulch near Crested Butte, Colorado, station 09111700.

[--, no data or not applicable; L, low; M, medium; H, high; NTRU, Nephelometric Turbidity Ratio Unit; E. coli, Escherichia coli; mg/m² milligrams per square meter; USEPA, U.S. Environmental Protection Agency; CDPHE, Colorado Department of Public Health and Environment; Cd(ch), Cadmium, chronic standard]

\begin{tabular}{|c|c|c|c|c|c|c|c|c|c|c|c|c|c|c|}
\hline $\begin{array}{l}\text { Property } \\
\text { or } \\
\text { constituent }\end{array}$ & $\begin{array}{l}\text { Period } \\
\text { (water } \\
\text { years) }\end{array}$ & $\begin{array}{c}\text { Number } \\
\text { of } \\
\text { samples }\end{array}$ & $\begin{array}{c}\text { Number } \\
\text { of } \\
\text { censored } \\
\text { values }\end{array}$ & Median' & Maximum & $\begin{array}{c}\text { Date } \\
\text { of } \\
\text { Maximum }\end{array}$ & $\begin{array}{c}\text { 15th } \\
\text { percentile }\end{array}$ & $\begin{array}{c}\text { 85th } \\
\text { percentile }\end{array}$ & $\begin{array}{l}\text { Geometric } \\
\text { Mean }^{2}\end{array}$ & $\begin{array}{c}\text { Standard } \\
\text { or } \\
\text { Chronic } \\
\text { standard }^{3}\end{array}$ & $\begin{array}{l}\text { Number of } \\
\text { exceedences } \\
\text { Standard or } \\
\text { Chronic }\end{array}$ & $\begin{array}{l}\text { Acute } \\
\text { standard }\end{array}$ & $\begin{array}{c}\text { Number of } \\
\text { exceedences } \\
\text { Acute }\end{array}$ & $\begin{array}{l}\text { Level } \\
\text { of } \\
\text { concern }\end{array}$ \\
\hline $\begin{array}{l}\text { Instantaneous discharge, in cubic feet } \\
\text { per second }\end{array}$ & 2007 & 5 & 0 & 23.4 & 342 & $06 / 13 / 07$ & 6.04 & 342 & -- & -- & -- & -- & -- & -- \\
\hline $\begin{array}{l}\text { Instantaneous discharge, in cubic feet } \\
\text { per second }\end{array}$ & 2008-2009 & 12 & 0 & 30.8 & 762 & $06 / 25 / 08$ & 12.8 & 536 & -- & -- & -- & -- & -- & -- \\
\hline Turbidity, in NTRU & 2007 & 4 & 2 & 2.8 & 5.8 & 06/13/07 & 0.0 & 5.8 & -- & -- & -- & -- & -- & -- \\
\hline Turbidity, in NTRU & 2008-2009 & 8 & 1 & 1.5 & 14.1 & $06 / 25 / 08$ & 0.4 & 12.5 & -- & -- & -- & -- & -- & -- \\
\hline $\begin{array}{l}\text { Dissolved oxygen, in milligrams per } \\
\text { liter }\end{array}$ & 2007 & 5 & 0 & 9.3 & 10.8 & $01 / 08 / 07$ & 7.2 & 10.8 & -- & 6.0 & 0 & -- & -- & $\mathrm{L}$ \\
\hline $\begin{array}{l}\text { Dissolved oxygen, in milligrams per } \\
\quad \text { liter }\end{array}$ & 2008-2009 & 12 & 0 & 8.9 & 10.5 & $11 / 20 / 08$ & 7.5 & 10.1 & -- & 6.0 & 0 & -- & -- & $\mathrm{L}$ \\
\hline $\mathrm{pH}$, in standard units & 2007 & 5 & 0 & 7.7 & 8.8 & $08 / 14 / 07$ & 7.6 & 8.8 & -- & 6.5 & 0 & 9.0 & -- & $\mathrm{L}$ \\
\hline $\mathrm{pH}$, in standard units & 2008-2009 & 12 & 0 & 7.7 & 8.5 & 09/18/08 & 7.2 & 8.4 & -- & 6.5 & 0 & 9.0 & -- & $\mathrm{L}$ \\
\hline $\begin{array}{l}\text { Specific conductance, in microsiemens } \\
\text { per centimeter }\end{array}$ & 2007 & 5 & 0 & 164 & 219 & 03/06/07 & 83.0 & 219 & -- & -- & -- & -- & -- & -- \\
\hline $\begin{array}{l}\text { Specific conductance, in microsiemens } \\
\text { per centimeter }\end{array}$ & 2008-2009 & 12 & 0 & 171 & 211 & $02 / 12 / 09$ & 90.3 & 205 & -- & -- & -- & -- & -- & -- \\
\hline Temperature, degrees Celsius & 2007 & 5 & 0 & 5.6 & 18.5 & $08 / 14 / 07$ & 0.0 & 18.5 & -- & 17.0 & 1 & -- & -- & $\mathrm{H}$ \\
\hline Temperature, degrees Celsius & $2008-2009$ & 12 & 0 & 8.0 & 17.9 & $08 / 19 / 08$ & 0.1 & 16.1 & -- & 17.0 & 1 & -- & -- & $\mathrm{L}$ \\
\hline Calcium, in milligrams per liter & 2007 & 4 & 0 & 21.8 & 27.3 & $01 / 08 / 07$ & 12.0 & 27.3 & -- & -- & -- & -- & -- & -- \\
\hline Calcium, in milligrams per liter & $2008-2009$ & 12 & 0 & 23.2 & 29.1 & 09/03/09 & 13.3 & 29.0 & -- & -- & -- & -- & -- & -- \\
\hline Magnesium, in milligrams per liter & 2007 & 4 & 0 & 3.29 & 4.03 & $01 / 08 / 07$ & 1.40 & 4.03 & -- & -- & -- & -- & -- & -- \\
\hline Magnesium, in milligrams per liter & 2008-2009 & 12 & 0 & 3.25 & 4.31 & $02 / 12 / 09$ & 1.55 & 4.31 & -- & -- & -- & -- & -- & -- \\
\hline $\begin{array}{l}\text { Ammonia plus organic nitrogen, in mil- } \\
\text { ligrams per liter }\end{array}$ & 2007 & 5 & 0 & 0.13 & 0.17 & $04 / 17 / 07$ & 0.07 & 0.17 & -- & -- & -- & -- & -- & -- \\
\hline $\begin{array}{l}\text { Ammonia plus organic nitrogen, in mil- } \\
\text { ligrams per liter }\end{array}$ & 2008-2009 & 12 & 0 & 0.12 & 0.52 & 05/08/08 & 0.08 & 0.25 & -- & -- & -- & -- & -- & -- \\
\hline $\begin{array}{l}\text { Ammonia nitrogen, in milligrams per } \\
\text { liter }\end{array}$ & 2007 & 5 & 4 & 4 & ${ }^{5}<0.02$ & -- & ${ }^{4}$ & ${ }^{4}$ & -- & 2.86 & 0 & 7.63 & 0 & $\mathrm{~L}$ \\
\hline $\begin{array}{l}\text { Ammonia nitrogen, in milligrams per } \\
\text { liter }\end{array}$ & 2008-2009 & 12 & 9 & ${ }^{4}$ & 0.039 & $02 / 12 / 09$ & 4 & 0.025 & -- & 3.45 & 0 & 10.8 & 0 & $\mathrm{~L}$ \\
\hline $\begin{array}{l}\text { Nitrite plus nitrate nitrogen, in mil- } \\
\text { ligrams per liter }\end{array}$ & 2007 & 5 & 0 & 0.123 & 0.342 & $01 / 08 / 07$ & 0.042 & 0.342 & -- & 10.0 & 0 & -- & -- & $\mathrm{L}$ \\
\hline $\begin{array}{l}\text { Nitrite plus nitrate nitrogen, in mil- } \\
\text { ligrams per liter }\end{array}$ & 2008-2009 & 12 & 0 & 0.091 & 0.313 & $12 / 18 / 07$ & 0.020 & 0.283 & -- & 10.0 & 0 & -- & -- & $\mathrm{L}$ \\
\hline Nitrite nitrogen, in milligrams per liter & 2007 & 5 & 1 & 0.002 & 0.005 & $01 / 08 / 07$ & 4 & 0.005 & -- & 0.05 & 0 & -- & -- & $\mathrm{L}$ \\
\hline Nitrite nitrogen, in milligrams per liter & 2008-2009 & 12 & 2 & 0.002 & 0.004 & $12 / 18 / 07$ & 4 & 0.002 & -- & 0.05 & 0 & -- & -- & $\mathrm{L}$ \\
\hline Orthophosphate, in milligrams per liter & 2007 & 5 & 0 & 0.012 & 0.014 & 03/06/07 & 0.005 & 0.014 & -- & -- & -- & -- & -- & -- \\
\hline
\end{tabular}


[--, no data or not applicable; L, low; M, medium; H, high; NTRU, Nephelometric Turbidity Ratio Unit; E. coli, Escherichia coli; mg/m², milligrams per square meter; USEPA, U.S. Environmental Protection Agency; CDPHE, Colorado Department of Public Health and Environment; Cd(ch), Cadmium, chronic standard]

\begin{tabular}{|c|c|c|c|c|c|c|c|c|c|c|c|c|c|c|}
\hline $\begin{array}{l}\text { Property } \\
\text { or } \\
\text { constituent }\end{array}$ & $\begin{array}{l}\text { Period } \\
\text { (water } \\
\text { years) }\end{array}$ & $\begin{array}{c}\text { Number } \\
\text { of } \\
\text { samples }\end{array}$ & $\begin{array}{c}\text { Number } \\
\text { of } \\
\text { censored } \\
\text { values }\end{array}$ & Median' & Maximum & $\begin{array}{c}\text { Date } \\
\text { of } \\
\text { Maximum }\end{array}$ & $\begin{array}{c}\text { 15th } \\
\text { percentile }\end{array}$ & $\begin{array}{c}\text { 85th } \\
\text { percentile }\end{array}$ & $\begin{array}{c}\text { Geometric } \\
\text { Mean }^{2}\end{array}$ & $\begin{array}{l}\text { Standard } \\
\text { or } \\
\text { Chronic } \\
\text { standard }^{3}\end{array}$ & $\begin{array}{l}\text { Number of } \\
\text { exceedences } \\
\text { Standard or } \\
\text { Chronic }\end{array}$ & $\begin{array}{l}\text { Acute } \\
\text { standard }\end{array}$ & $\begin{array}{l}\text { Number of } \\
\text { exceedences } \\
\text { Acute }\end{array}$ & $\begin{array}{c}\text { Level } \\
\text { of } \\
\text { concern }\end{array}$ \\
\hline Orthophosphate, in milligrams per liter & 2008-2009 & 12 & 3 & 0.010 & 0.028 & $02 / 12 / 09$ & 4 & 0.027 & -- & -- & -- & -- & -- & -- \\
\hline $\begin{array}{l}\text { Phosphorus (total), in milligrams per } \\
\text { liter }\end{array}$ & 2007 & 5 & 0 & 0.030 & 0.032 & 03/06/07 & 0.021 & 0.032 & -- & -- & -- & -- & -- & -- \\
\hline $\begin{array}{l}\text { Phosphorus (total), in milligrams per } \\
\text { liter }\end{array}$ & 2008-2009 & 12 & 0 & 0.031 & 0.167 & 05/08/08 & 0.021 & 0.052 & -- & -- & -- & -- & -- & -- \\
\hline Organic carbon, in milligrams per liter & 2007 & 2 & 0 & 2.2 & 2.3 & $04 / 17 / 07$ & 2.0 & 2.3 & -- & -- & -- & -- & -- & -- \\
\hline Organic carbon, in milligrams per liter & $2008-2008$ & 2 & 0 & 2.1 & 3.0 & 05/08/08 & 1.2 & 3.0 & -- & -- & -- & -- & -- & -- \\
\hline E. coli, in colonies per 100 milliliter & 2007 & 5 & 2 & 2 & 86 & $08 / 14 / 07$ & 1 & 6 & 5.8 & 126 & 0 & -- & -- & $\mathrm{L}$ \\
\hline E. coli, in colonies per 100 milliliter & $2008-2009$ & 11 & 2 & 11 & 33 & 09/03/09 & 1 & 6 & -- & 126 & 0 & -- & -- & $\mathrm{L}$ \\
\hline $\begin{array}{l}\text { Biomass periphyton, ashfree drymass, } \\
\text { in grams per square meter }\end{array}$ & 2007 & 1 & 0 & 10.9 & 10.9 & $08 / 14 / 07$ & 10.9 & 10.9 & -- & -- & -- & -- & -- & -- \\
\hline $\begin{array}{l}\text { Biomass periphyton, ashfree drymass, } \\
\text { in grams per square meter }\end{array}$ & $2008-2009$ & 2 & 1 & 7.3 & 14.6 & 09/03/09 & 4 & 14.6 & -- & -- & -- & -- & -- & -- \\
\hline $\begin{array}{l}\text { Periphyton, biomass, ash weight, in } \\
\text { grams per square meter }\end{array}$ & 2007 & 1 & 0 & 375 & 375 & $08 / 14 / 07$ & 375 & 375 & -- & -- & -- & -- & -- & -- \\
\hline $\begin{array}{l}\text { Periphyton, biomass, ash weight, in } \\
\text { grams per square meter }\end{array}$ & 2008-2009 & 2 & 0 & 423 & 631 & 09/03/09 & 214 & 631 & -- & -- & -- & -- & -- & -- \\
\hline $\begin{array}{l}\text { Periphyton, biomass, dry weight, in } \\
\text { grams per square meter }\end{array}$ & 2007 & 1 & 0 & 386 & 386 & $08 / 14 / 07$ & 386 & 386 & -- & -- & -- & -- & -- & -- \\
\hline $\begin{array}{l}\text { Periphyton, biomass, dry weight, in } \\
\text { grams per square meter }\end{array}$ & 2008-2009 & 2 & 0 & 432 & 646 & 09/03/09 & 218 & 646 & -- & -- & -- & -- & -- & -- \\
\hline $\begin{array}{l}\text { Chlorphyll a, periphyton, chromofluoro, } \\
\text { in } \mathrm{mg} / \mathrm{m}^{2}\end{array}$ & 2007 & 1 & 0 & 19.9 & 19.9 & $08 / 14 / 07$ & 19.9 & 19.9 & -- & -- & -- & -- & -- & -- \\
\hline $\begin{array}{l}\text { Chlorphyll a, periphyton, chromofluoro, } \\
\text { in } \mathrm{mg} / \mathrm{m}^{2}\end{array}$ & 2008-2009 & 2 & 0 & 9.99 & 12.2 & $09 / 18 / 08$ & 7.74 & 12.2 & -- & -- & -- & -- & -- & -- \\
\hline Pheophytin a, periphyton, in $\mathrm{mg} / \mathrm{m}^{2}$ & 2007 & 1 & 0 & 12.8 & 12.8 & 08/14/07 & 12.8 & 12.8 & -- & -- & -- & -- & -- & -- \\
\hline Pheophytin a, periphyton, in $\mathrm{mg} / \mathrm{m}^{2}$ & $2008-2009$ & 2 & 0 & 5.8 & 8.3 & 09/18/08 & 3.3 & 8.3 & -- & -- & -- & -- & -- & -- \\
\hline Aluminum, in micrograms per liter & 2007 & 4 & 0 & 19.5 & 25.5 & 06/13/07 & 4.1 & 25.5 & -- & -- & -- & -- & -- & -- \\
\hline Aluminum, in micrograms per liter & 2008-2009 & 7 & 0 & 7.1 & 75.0 & 05/04/09 & 2.1 & 68.1 & -- & -- & -- & -- & -- & -- \\
\hline Cadmium, in micrograms per liter & 2007 & 4 & 0 & 0.22 & 0.29 & 06/13/07 & 0.06 & 0.29 & -- & 70.31 & 0 & 1.20 & 0 & M \\
\hline Cadmium, in micrograms per liter & 2008-2009 & 7 & 0 & 0.15 & 0.65 & 05/04/09 & 0.10 & 0.56 & -- & ${ }^{7} 0.31$ & 1 & 1.20 & 0 & $\mathrm{H}$ \\
\hline Copper, in micrograms per liter & 2007 & 4 & 0 & 2.3 & 3.3 & 06/13/07 & 1.8 & 3.3 & -- & 6.4 & 0 & 9.2 & 0 & M \\
\hline Copper, in micrograms per liter & 2008-2009 & 7 & 1 & 0.7 & 2.7 & 05/04/09 & 0.10 & 2.5 & -- & 6.4 & 0 & 9.2 & 0 & $\mathrm{~L}$ \\
\hline Iron, in micrograms per liter & 2007 & 4 & 0 & 32 & 48 & 06/13/07 & 17 & 48 & -- & 300 & 0 & -- & -- & $\mathrm{L}$ \\
\hline Iron, in micrograms per liter & 2008-2009 & 7 & 0 & 58 & 73 & 05/04/09 & 29 & 71 & -- & 300 & 0 & -- & -- & $\mathrm{L}$ \\
\hline Lead, in micrograms per liter & 2007 & 4 & 0 & 0.36 & 1.07 & $06 / 13 / 07$ & 0.11 & 1.07 & -- & 1.62 & 0 & 41.7 & 0 & M \\
\hline
\end{tabular}


Table 7. Summary of measured constituents and properties for Slate River above Squaw Gulch near Crested Butte, Colorado, station 09111700._Continued

[--, no data or not applicable; L, low; M, medium; H, high; NTRU, Nephelometric Turbidity Ratio Unit; E. coli, Escherichia coli; mg/m² milligrams per square meter; USEPA, U.S. Environmental Protection Agency; CDPHE, Colorado Department of Public Health and Environment; Cd(ch), Cadmium, chronic standard]

\begin{tabular}{|c|c|c|c|c|c|c|c|c|c|c|c|c|c|c|}
\hline $\begin{array}{l}\text { Property } \\
\text { or } \\
\text { constituent }\end{array}$ & $\begin{array}{l}\text { Period } \\
\text { (water } \\
\text { years) }\end{array}$ & $\begin{array}{l}\text { Number } \\
\text { of } \\
\text { samples }\end{array}$ & $\begin{array}{c}\text { Number } \\
\text { of } \\
\text { censored } \\
\text { values }\end{array}$ & Median $^{1}$ & Maximum & $\begin{array}{c}\text { Date } \\
\text { of } \\
\text { Maximum }\end{array}$ & $\begin{array}{c}\text { 15th } \\
\text { percentile }\end{array}$ & $\begin{array}{c}\text { 85th } \\
\text { percentile }\end{array}$ & $\begin{array}{c}\text { Geometric } \\
\text { Mean }^{2}\end{array}$ & $\begin{array}{l}\text { Standard } \\
\text { or } \\
\text { Chronic } \\
\text { standard }\end{array}$ & $\begin{array}{l}\text { Number of } \\
\text { exceedences } \\
\text { Standard or } \\
\text { Chronic }\end{array}$ & $\begin{array}{l}\text { Acute } \\
\text { standard }\end{array}$ & $\begin{array}{l}\text { Number of } \\
\text { exceedences } \\
\text { Acute }\end{array}$ & $\begin{array}{l}\text { Level } \\
\text { of } \\
\text { concern }\end{array}$ \\
\hline Lead, in micrograms per liter & $2008-2009$ & 7 & 0 & 0.32 & 0.59 & $06 / 25 / 08$ & 0.05 & 0.57 & -- & 1.62 & 0 & 41.7 & 0 & $\mathrm{~L}$ \\
\hline Manganese, in micrograms per liter & 2007 & 4 & 0 & 35.1 & 57.2 & $01 / 08 / 07$ & 9.2 & 57.2 & -- & 1,444 & 0 & 2,613 & 0 & $\mathrm{~L}$ \\
\hline Manganese, in micrograms per liter & 2008-2009 & 7 & 0 & 32.0 & 41.3 & $10 / 17 / 07$ & 13.9 & 41.2 & -- & 1,444 & 0 & 2,613 & 0 & $\mathrm{~L}$ \\
\hline Silver, in micrograms per liter & 2007 & 4 & 4 & 4 & ${ }^{5}<0.1$ & -- & 4 & 4 & -- & 0.04 & 0 & 1.02 & 0 & $\mathrm{~L}$ \\
\hline Silver, in micrograms per liter & $2008-2009$ & 7 & 6 & 4 & ${ }^{5}<0.1$ & -- & 4 & 4 & -- & 0.04 & 0 & 1.02 & 0 & $\mathrm{~L}$ \\
\hline Zinc, in micrograms per liter & 2007 & 4 & 0 & 50.8 & 61.0 & $04 / 17 / 07$ & 6.44 & 61.0 & -- & 88.4 & 0 & 102 & 0 & M \\
\hline Zinc, in micrograms per liter & 2008-2009 & 7 & 0 & 26.4 & 103 & 05/04/09 & 8.97 & 91.5 & -- & 88.4 & 1 & 102 & 1 & $\mathrm{H}$ \\
\hline Benzene (total), in micrograms per liter & 2007 & 1 & 1 & 4 & ${ }^{s}<0.016$ & -- & 4 & 4 & -- & -- & -- & -- & -- & -- \\
\hline Benzene (total), in micrograms per liter & $2008-2008$ & 1 & 1 & 4 & ${ }^{s}<0.02$ & -- & 4 & 4 & -- & -- & -- & -- & -- & -- \\
\hline $\begin{array}{l}\text { Ethylbenzene (total), in micrograms } \\
\text { per liter }\end{array}$ & 2007 & 1 & 1 & 4 & ${ }^{5}<0.02$ & -- & 4 & 4 & -- & -- & -- & -- & -- & -- \\
\hline $\begin{array}{l}\text { Ethylbenzene (total), in micrograms } \\
\text { per liter }\end{array}$ & $2008-2008$ & 1 & 1 & 4 & ${ }^{5}<0.04$ & -- & 4 & 4 & -- & -- & -- & -- & -- & -- \\
\hline $\begin{array}{l}\text { m- + p-Xylene (total) in micrograms } \\
\text { per liter }\end{array}$ & 2007 & 1 & 1 & 4 & ${ }^{5}<0.08$ & -- & 4 & 4 & -- & -- & -- & -- & -- & -- \\
\hline $\begin{array}{l}\mathrm{m}-+ \text { p-Xylene (total) in micrograms } \\
\text { per liter }\end{array}$ & $2008-2008$ & 1 & 1 & 4 & ${ }^{5}<0.08$ & -- & 4 & 4 & -- & -- & -- & -- & -- & -- \\
\hline o-Xylene (total), in micrograms per liter & 2007 & 1 & 1 & 4 & ${ }^{s}<0.04$ & -- & 4 & 4 & -- & -- & -- & -- & -- & -- \\
\hline o-Xylene (total), in micrograms per liter & $2008-2008$ & 1 & 1 & 4 & ${ }^{s<0.04}$ & -- & 4 & 4 & -- & -- & -- & -- & -- & -- \\
\hline Toluene (total), in micrograms per liter & 2007 & 1 & 0 & 4 & ${ }^{5}<0.02$ & -- & 4 & 4 & -- & -- & -- & -- & -- & -- \\
\hline Toluene (total), in micrograms per liter & $2008-2008$ & 1 & 1 & 4 & ${ }^{5}<0.02$ & -- & 4 & 4 & -- & -- & -- & -- & -- & -- \\
\hline $\begin{array}{l}\text { Suspended sediment, in milligrams } \\
\text { per liter }\end{array}$ & 2007 & 4 & 0 & 5.5 & 16.0 & 06/13/07 & 1.0 & 16.0 & -- & -- & -- & -- & -- & -- \\
\hline $\begin{array}{l}\text { Suspended sediment, in milligrams } \\
\text { per liter }\end{array}$ & 2008-2009 & 8 & 0 & 3.0 & 116 & 05/08/08 & 1.0 & 84.2 & -- & -- & -- & -- & -- & -- \\
\hline
\end{tabular}

${ }^{1}$ Censored values were replaced with 0 to compute median and 85 th percentiles (coliform censored values replaced with 1 ) see definitions section.

${ }^{2}$ Geometric mean listed for E. coli. Geometric mean computed for combined period of record (2007-2009).

${ }^{3}$ Colorado Department of Public Health and Environment, classification and numeric standards for Gunnison and Lower Dolores River Basins (2010a); and USEPA National Recommended Water Quality Criteria (2009). ${ }^{4}$ Median values and percentiles were not tabled when they were calculated as zero.

${ }^{5}$ All values below laboratory reporting level (LRL), current LRL used for current period maximum, highest LRL used for historic period.

${ }^{6}$ Use geometric mean for comparison to standard.

${ }^{7}$ Temporary modifications to CDPHE numeric standards for this stream segment: $\mathrm{Cd}(\mathrm{ch})=0.4$ Expiration Date of 12/31/2012. 


\section{Taylor River at Taylor Park, Colorado}

Current Reason for Inclusion: To collect baseline data. This station is part of the rotational group of sites.

General Station Information:

Location:

0.2 mi upstream from Taylor Park Reservoir waterline

(2.7 mi north of Taylor Park, and 21 mi northeast of Almont).

Station Type:

USGS water quality and streamflow gaging

Period of Record:

Water quality: January 2007-September 2009

Streamflow gaging:

June 1929-September 1934, October 1987-September 2009

Latitude: 385137

Longitude: 1063358

Drainage area: $128 \mathrm{mi}^{2}$

HUC: 14020002

Stream segment: 4

USGS Data Summary: Water Years 2008-2009:

General Chemistry:

$\mathrm{pH}$ : Low concern

Dissolved oxygen: Low concern

Temperature: Low concern

Nutrients:

Low concern

E. coli:

Low concern

Trace Elements/metals:

Medium concern: Iron

Other constituents of concern: None for constituents tested.

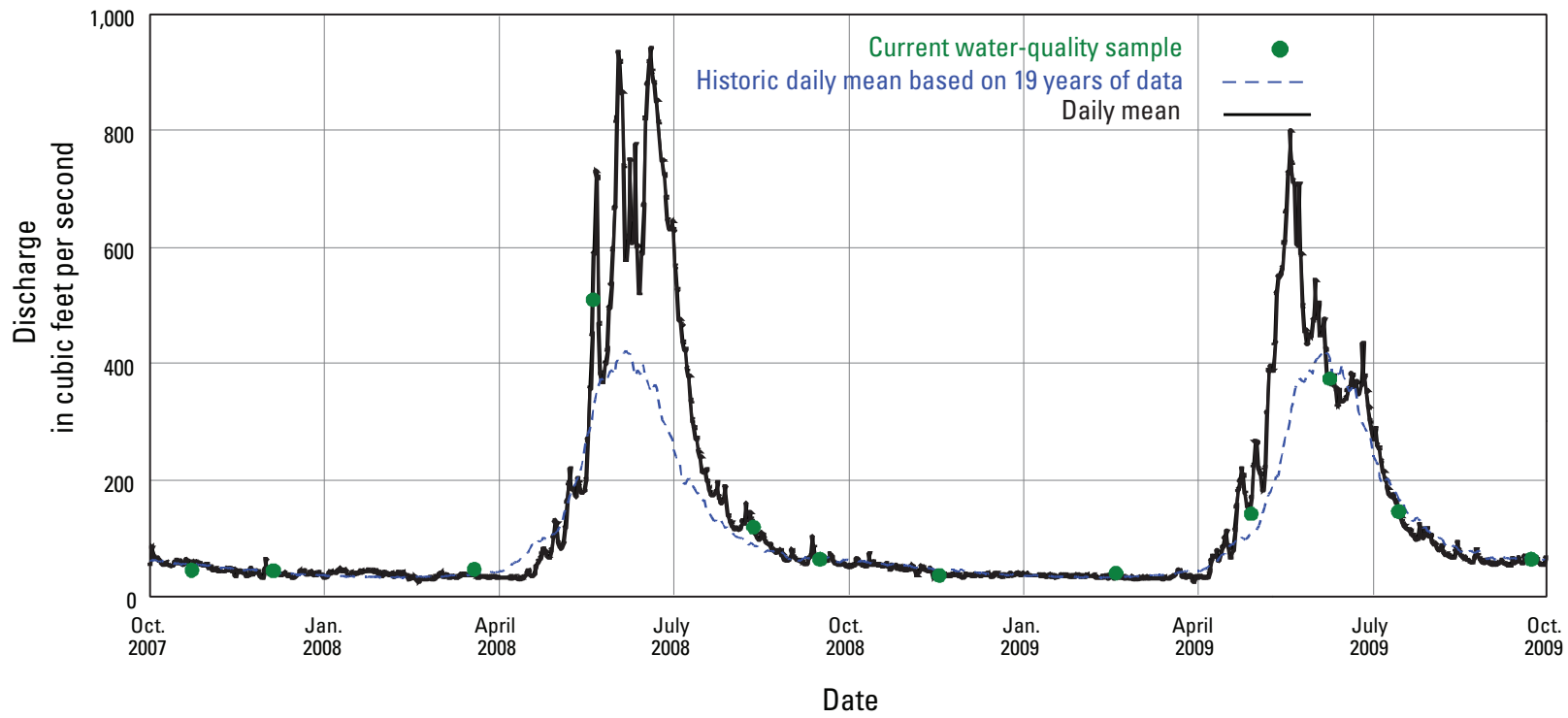

Figure 10. Daily mean discharge and time distribution of water-quality samples for Taylor River at Taylor Park, Colorado. 

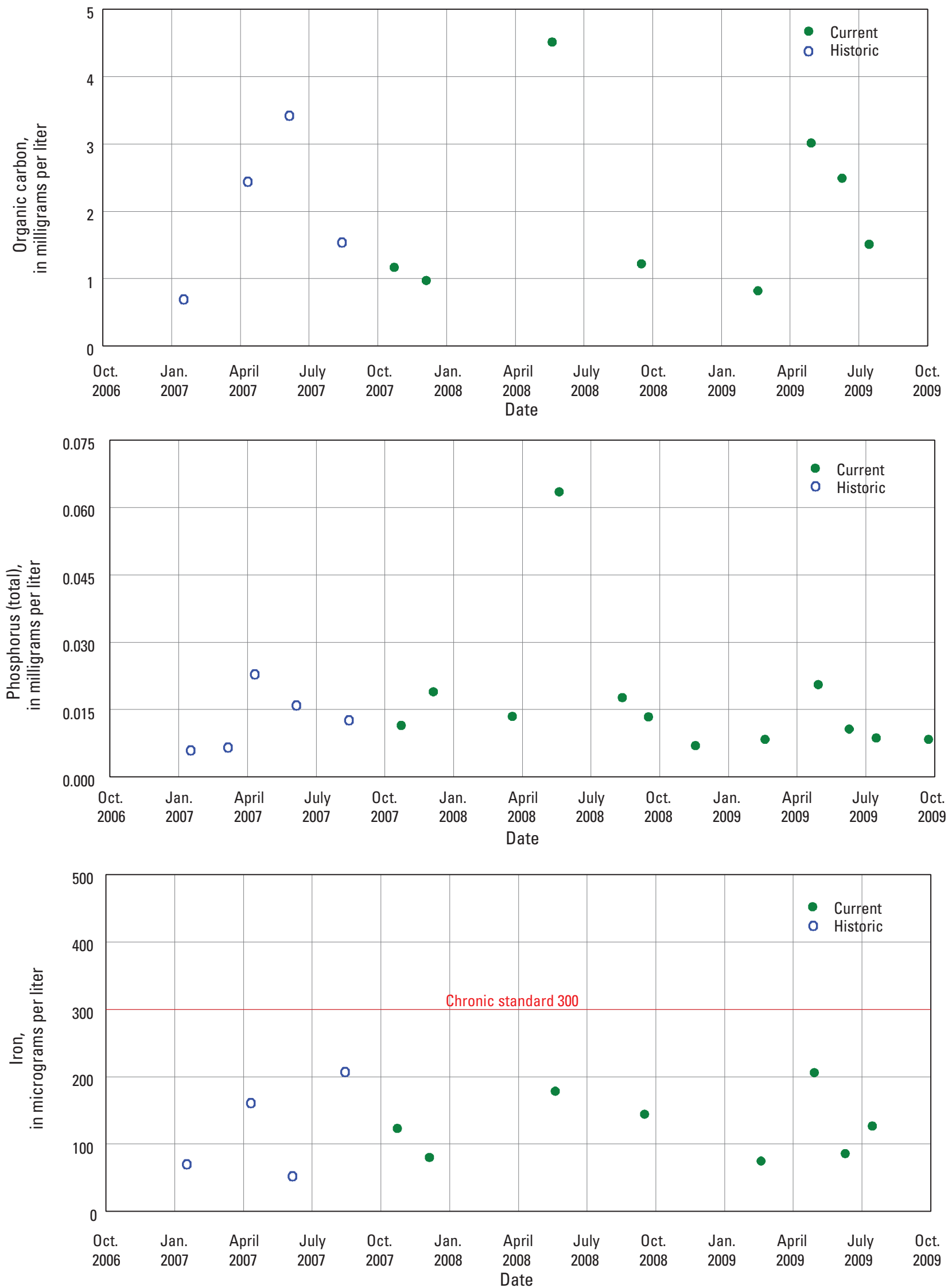

Figure 11. Time-series plots of selected water-quality constituents for Taylor River at Taylor Park, Colorado. 
[--, no data or not applicable; L, low; M, medium; H, high; NTRU, Nephelometric Turbidity Ratio Unit; E. coli, Escherichia coli; mg/m², milligrams per square meter; USEPA, U.S. Environmental Protection Agency]

\begin{tabular}{|c|c|c|c|c|c|c|c|c|c|c|c|c|c|c|}
\hline $\begin{array}{l}\text { Property } \\
\text { or } \\
\text { constituent }\end{array}$ & $\begin{array}{l}\text { Period } \\
\text { (water } \\
\text { years) }\end{array}$ & $\begin{array}{l}\text { Number } \\
\text { of } \\
\text { samples }\end{array}$ & $\begin{array}{c}\text { Number } \\
\text { of } \\
\text { censored } \\
\text { values }\end{array}$ & Median' & Maximum & $\begin{array}{c}\text { Date } \\
\text { of } \\
\text { Maximum }\end{array}$ & $\begin{array}{c}\text { 15th } \\
\text { percentile }\end{array}$ & $\begin{array}{c}\text { 85th } \\
\text { percentile }\end{array}$ & $\begin{array}{c}\text { Geometric } \\
\text { Mean }^{2}\end{array}$ & $\begin{array}{l}\text { Standard } \\
\text { or } \\
\text { Chronic } \\
\text { standard }\end{array}$ & $\begin{array}{l}\text { Number of } \\
\text { exceedences } \\
\text { Standard or } \\
\text { Chronic }\end{array}$ & $\begin{array}{l}\text { Acute } \\
\text { standard }\end{array}$ & $\begin{array}{c}\text { Number of } \\
\text { exceedences } \\
\text { Acute }\end{array}$ & $\begin{array}{l}\text { Level of } \\
\text { concern }\end{array}$ \\
\hline $\begin{array}{l}\text { Instantaneous discharge, in } \\
\text { cubic feet per second }\end{array}$ & 2007 & 5 & 0 & 50 & 389 & 06/05/07 & 39 & 389 & -- & -- & -- & -- & -- & -- \\
\hline $\begin{array}{l}\text { Instantaneous discharge, in } \\
\text { cubic feet per second }\end{array}$ & 2008-2009 & 12 & 0 & 64 & 510 & $05 / 20 / 08$ & 40 & 381 & -- & -- & -- & -- & -- & -- \\
\hline Turbidity, in NTRU & 2007 & 4 & 2 & 1.0 & 2.8 & $06 / 05 / 07$ & 0.0 & 2.8 & -- & -- & -- & -- & -- & -- \\
\hline Turbidity, in NTRU & 2008-2009 & 8 & 1 & 2.1 & 6.7 & $05 / 20 / 08$ & 0.4 & 6.0 & -- & -- & -- & -- & -- & -- \\
\hline $\begin{array}{l}\text { Dissolved oxygen, in mil- } \\
\text { ligrams per liter }\end{array}$ & 2007 & 5 & 0 & 9.8 & 11.0 & $01 / 17 / 07$ & 7.3 & 11.0 & -- & 6.0 & 0 & -- & -- & $\mathrm{L}$ \\
\hline $\begin{array}{l}\text { Dissolved oxygen, in mil- } \\
\text { ligrams per liter }\end{array}$ & 2008-2009 & 12 & 0 & 9.5 & 11.0 & 03/19/08 & 8.1 & 10.9 & -- & 6.0 & 0 & -- & -- & $\mathrm{L}$ \\
\hline $\mathrm{pH}$, in standard units & 2007 & 5 & 0 & 8.6 & 8.8 & $03 / 07 / 07$ & 8.0 & 8.8 & -- & 6.5 & 0 & 9.0 & -- & $\mathrm{L}$ \\
\hline $\mathrm{pH}$, in standard units & 2008-2009 & 12 & 0 & 8.0 & 8.4 & $11 / 18 / 08$ & 7.4 & 8.3 & -- & 6.5 & 0 & 9.0 & -- & $\mathrm{L}$ \\
\hline $\begin{array}{l}\text { Specific conductance, in micro- } \\
\text { siemens per centimeter }\end{array}$ & 2007 & 5 & 0 & 115 & 123 & $01 / 17 / 07$ & 75 & 123 & -- & -- & -- & -- & -- & -- \\
\hline $\begin{array}{l}\text { Specific conductance, in micro- } \\
\text { siemens per centimeter }\end{array}$ & 2008-2009 & 12 & 0 & 113 & 119 & $12 / 05 / 07$ & 79 & 118 & -- & -- & -- & -- & -- & -- \\
\hline Temperature, degrees Celsius & 2007 & 5 & 0 & 3.5 & 16.0 & $08 / 14 / 07$ & 0.1 & 16.0 & -- & 17.0 & 0 & -- & -- & $\mathrm{L}$ \\
\hline Temperature, degrees Celsius & 2008-2009 & 12 & 0 & 6.2 & 15.2 & $08 / 12 / 08$ & 4 & 11.1 & -- & 17.0 & 0 & -- & -- & $\mathrm{L}$ \\
\hline Calcium, in milligrams per liter & 2007 & 4 & 0 & 13.9 & 15.5 & $01 / 17 / 07$ & 9.67 & 15.5 & -- & -- & -- & -- & -- & -- \\
\hline Calcium, in milligrams per liter & 2008-2009 & 8 & 0 & 14.0 & 15.6 & $10 / 23 / 07$ & 8.60 & 15.6 & -- & -- & -- & -- & -- & -- \\
\hline $\begin{array}{l}\text { Magnesium, in milligrams } \\
\text { per liter }\end{array}$ & 2007 & 4 & 0 & 3.28 & 3.92 & $01 / 17 / 07$ & 2.33 & 3.92 & -- & -- & -- & -- & -- & -- \\
\hline $\begin{array}{l}\text { Magnesium, in milligrams } \\
\text { per liter }\end{array}$ & 2008-2009 & 8 & 0 & 3.35 & 3.78 & $12 / 05 / 07$ & 2.12 & 3.75 & -- & -- & -- & -- & -- & -- \\
\hline $\begin{array}{l}\text { Ammonia plus organic nitro- } \\
\text { gen, in milligrams per liter }\end{array}$ & 2007 & 5 & 1 & 0.12 & 0.20 & 04/11/07 & 4 & 0.20 & -- & -- & -- & -- & -- & -- \\
\hline $\begin{array}{l}\text { Ammonia plus organic nitro- } \\
\text { gen, in milligrams per liter }\end{array}$ & $2008-2009$ & 12 & 0 & 0.10 & 0.36 & $05 / 20 / 08$ & 0.070 & 0.20 & -- & -- & -- & -- & -- & -- \\
\hline $\begin{array}{l}\text { Ammonia nitrogen, in mil- } \\
\text { ligrams per liter }\end{array}$ & 2007 & 5 & 4 & 4 & ${ }^{s}<0.02$ & -- & 4 & 4 & -- & 1.22 & 0 & 2.64 & 0 & $\mathrm{~L}$ \\
\hline $\begin{array}{l}\text { Ammonia nitrogen, in mil- } \\
\text { ligrams per liter }\end{array}$ & $2008-2009$ & 12 & 11 & 4 & ${ }^{s}<0.02$ & -- & 4 & 4 & -- & 2.63 & 0 & 7.01 & 0 & $\mathrm{~L}$ \\
\hline $\begin{array}{l}\text { Nitrite plus nitrate nitrogen, in } \\
\text { milligrams per liter }\end{array}$ & 2007 & 5 & 3 & 4 & 0.027 & $01 / 17 / 07$ & 4 & 0.027 & -- & 10.0 & 0 & -- & -- & $\mathrm{L}$ \\
\hline $\begin{array}{l}\text { Nitrite plus nitrate nitrogen, in } \\
\text { milligrams per liter }\end{array}$ & 2008-2009 & 12 & 3 & 0.013 & 0.042 & $02 / 18 / 09$ & 4 & 0.037 & -- & 10.0 & 0 & -- & -- & $\mathrm{L}$ \\
\hline $\begin{array}{l}\text { Nitrite nitrogen, in milligrams } \\
\text { per liter }\end{array}$ & 2007 & 5 & 3 & 4 & ${ }^{s}<0.002$ & -- & 4 & 4 & -- & 0.05 & 0 & -- & -- & $\mathrm{L}$ \\
\hline
\end{tabular}


Table 8. Summary of measured constituents and properties for Taylor River at Taylor Park, Colorado, station 09107000.—Continued

[--, no data or not applicable; L, low; M, medium; H, high; NTRU, Nephelometric Turbidity Ratio Unit; E. coli, Escherichia coli; mg/m² , milligrams per square meter; USEPA, U.S. Environmental Protection Agency]

\begin{tabular}{|c|c|c|c|c|c|c|c|c|c|c|c|c|c|c|}
\hline $\begin{array}{l}\text { Property } \\
\text { or } \\
\text { constituent }\end{array}$ & $\begin{array}{l}\text { Period } \\
\text { (water } \\
\text { years) }\end{array}$ & $\begin{array}{c}\text { Number } \\
\text { of } \\
\text { samples }\end{array}$ & $\begin{array}{c}\begin{array}{c}\text { Number } \\
\text { of }\end{array} \\
\text { censored } \\
\text { values }\end{array}$ & Median' & Maximum & $\begin{array}{c}\text { Date } \\
\text { of } \\
\text { Maximum }\end{array}$ & $\begin{array}{c}\text { 15th } \\
\text { percentile }\end{array}$ & $\begin{array}{c}\text { 85th } \\
\text { percentile }\end{array}$ & $\begin{array}{c}\text { Geometric } \\
\text { Mean }^{2}\end{array}$ & $\begin{array}{c}\text { Standard } \\
\text { or } \\
\text { Chronic } \\
\text { standard }^{3}\end{array}$ & $\begin{array}{l}\text { Number of } \\
\text { exceedences } \\
\text { Standard or } \\
\text { Chronic }\end{array}$ & $\begin{array}{c}\text { Acute } \\
\text { standard }\end{array}$ & $\begin{array}{l}\text { Number of } \\
\text { exceedences } \\
\text { Acute }\end{array}$ & $\begin{array}{l}\text { Level of } \\
\text { concern }\end{array}$ \\
\hline $\begin{array}{l}\text { Nitrite nitrogen, in milligrams } \\
\text { per liter }\end{array}$ & 2008-2009 & 12 & 11 & 4 & ${ }^{5}<0.002$ & -- & 4 & 4 & -- & 0.05 & 0 & -- & -- & $\mathrm{L}$ \\
\hline $\begin{array}{l}\text { Orthophosphate, in milligrams } \\
\text { per liter }\end{array}$ & 2007 & 5 & 3 & 4 & 0.007 & 08/14/07 & 4 & 0.007 & -- & -- & -- & -- & -- & -- \\
\hline $\begin{array}{l}\text { Orthophosphate, in milligrams } \\
\text { per liter }\end{array}$ & 2008-2009 & 12 & 0 & 0.005 & 0.008 & 04/29/09 & 0.003 & 0.007 & -- & -- & -- & -- & -- & -- \\
\hline $\begin{array}{l}\text { Phosphorus (total), in mil- } \\
\text { ligrams per liter }\end{array}$ & 2007 & 5 & 0 & 0.013 & 0.023 & 04/11/07 & 0.006 & 0.023 & -- & -- & -- & -- & -- & -- \\
\hline $\begin{array}{l}\text { Phosphorus (total), in mil- } \\
\text { ligrams per liter }\end{array}$ & 2008-2009 & 12 & 0 & 0.012 & 0.064 & $05 / 20 / 08$ & 0.008 & 0.023 & -- & -- & -- & -- & -- & -- \\
\hline $\begin{array}{l}\text { Organic carbon, in milligrams } \\
\text { per liter }\end{array}$ & 2007 & 4 & 0 & 2.0 & 3.4 & 06/05/07 & 0.69 & 3.4 & -- & -- & -- & -- & -- & -- \\
\hline $\begin{array}{l}\text { Organic carbon, in milligrams } \\
\text { per liter }\end{array}$ & 2008-2009 & 8 & 0 & 1.4 & 4.5 & 05/20/08 & 0.88 & 4.0 & -- & -- & -- & -- & -- & -- \\
\hline $\begin{array}{l}\text { E. coli, in colonies per } 100 \\
\text { milliliter }\end{array}$ & 2007 & 5 & 3 & 1 & 6 & $08 / 14 / 07$ & 1 & 6 & 1.7 & 126 & 0 & -- & -- & L \\
\hline $\begin{array}{l}\text { E. coli, in colonies per } 100 \\
\text { milliliter }\end{array}$ & 2008-2009 & 11 & 5 & 1 & 6 & 07/15/09 & 1 & 6 & -- & 126 & 0 & -- & -- & $\mathrm{L}$ \\
\hline $\begin{array}{l}\text { Biomass periphyton, ashfree } \\
\text { drymass, in grams per } \\
\text { square meter }\end{array}$ & 2007 & 1 & 0 & -- & 16.6 & 08/14/07 & -- & -- & -- & -- & -- & -- & -- & -- \\
\hline $\begin{array}{l}\text { Biomass periphyton, ashfree } \\
\text { drymass, in grams per } \\
\text { square meter }\end{array}$ & 2008-2009 & 2 & 1 & 4.7 & 9.4 & 09/23/09 & 4 & 9.4 & -- & -- & -- & -- & -- & -- \\
\hline $\begin{array}{l}\text { Periphyton, biomass, ash } \\
\text { weight, in grams per square } \\
\text { meter }\end{array}$ & 2007 & 1 & 0 & -- & 451 & 08/14/07 & -- & -- & -- & -- & -- & -- & -- & -- \\
\hline $\begin{array}{l}\text { Periphyton, biomass, ash } \\
\text { weight, in grams per square } \\
\text { meter }\end{array}$ & 2008-2009 & 2 & 0 & 291 & 322 & 09/23/09 & 261 & 322 & -- & -- & -- & -- & -- & -- \\
\hline $\begin{array}{l}\text { Periphyton, biomass, dry } \\
\text { weight, in grams per square } \\
\text { meter }\end{array}$ & 2007 & 1 & 0 & -- & 468 & 08/14/07 & -- & -- & -- & -- & -- & -- & -- & -- \\
\hline $\begin{array}{l}\text { Periphyton, biomass, dry } \\
\text { weight, in grams per square } \\
\text { meter }\end{array}$ & 2008-2009 & 2 & 0 & 298 & 332 & 09/23/09 & 265 & 332 & -- & -- & -- & -- & -- & -- \\
\hline $\begin{array}{l}\text { Chlorophyll a, periphyton, } \\
\text { chromofluoro, in } \mathrm{mg} / \mathrm{m}^{2}\end{array}$ & 2007 & 1 & 0 & -- & 12.5 & 08/14/07 & -- & -- & -- & -- & -- & -- & -- & -- \\
\hline $\begin{array}{l}\text { Chlorophyll a, periphyton, } \\
\text { chromofluoro, in } \mathrm{mg} / \mathrm{m}^{2}\end{array}$ & 2008-2009 & 2 & 0 & 18.9 & 25.5 & 09/23/09 & 12.4 & 25.5 & -- & -- & -- & -- & -- & -- \\
\hline
\end{tabular}


[--, no data or not applicable; L, low; M, medium; H, high; NTRU, Nephelometric Turbidity Ratio Unit; E. coli, Escherichia coli; mg/m², milligrams per square meter; USEPA, U.S. Environmental Protection Agency]

\begin{tabular}{|c|c|c|c|c|c|c|c|c|c|c|c|c|c|c|}
\hline $\begin{array}{l}\text { Property } \\
\text { or } \\
\text { constituent }\end{array}$ & $\begin{array}{l}\text { Period } \\
\text { (water } \\
\text { years) }\end{array}$ & $\begin{array}{l}\text { Number } \\
\text { of } \\
\text { samples }\end{array}$ & $\begin{array}{c}\text { Number } \\
\text { of } \\
\text { censored } \\
\text { values }\end{array}$ & Median' & Maximum & $\begin{array}{c}\text { Date } \\
\text { of } \\
\text { Maximum }\end{array}$ & $\begin{array}{c}\text { 15th } \\
\text { percentile }\end{array}$ & $\begin{array}{c}\text { 85th } \\
\text { percentile }\end{array}$ & $\begin{array}{l}\text { Geometric } \\
\text { Mean }^{2}\end{array}$ & $\begin{array}{c}\text { Standard } \\
\text { or } \\
\text { Chronic } \\
\text { standard }^{3} \\
\end{array}$ & $\begin{array}{l}\text { Number of } \\
\text { exceedences } \\
\text { Standard or } \\
\text { Chronic }\end{array}$ & $\begin{array}{l}\text { Acute } \\
\text { standard }\end{array}$ & $\begin{array}{l}\text { Number of } \\
\text { exceedences } \\
\text { Acute }\end{array}$ & $\begin{array}{l}\text { Level of } \\
\text { concern }\end{array}$ \\
\hline $\begin{array}{l}\text { Pheophytin a, periphyton, in } \\
\mathrm{mg} / \mathrm{m}^{2}\end{array}$ & 2007 & 1 & 0 & -- & 9.9 & $08 / 14 / 07$ & -- & -- & -- & -- & -- & -- & -- & -- \\
\hline $\begin{array}{l}\text { Pheophytin a, periphyton, in } \\
\mathrm{mg} / \mathrm{m}^{2}\end{array}$ & 2008-2009 & 2 & 0 & 7.0 & 9.6 & 09/23/09 & 4.3 & 9.6 & -- & -- & -- & -- & -- & -- \\
\hline $\begin{array}{l}\text { Aluminum, in micrograms } \\
\text { per liter }\end{array}$ & 2007 & 4 & 0 & 7.4 & 16.5 & 06/05/07 & 3.8 & 16.5 & -- & -- & -- & -- & -- & -- \\
\hline $\begin{array}{l}\text { Aluminum, in micrograms } \\
\text { per liter }\end{array}$ & 2008-2009 & 8 & 0 & 7.6 & 46.0 & $05 / 20 / 08$ & 2.7 & 41.8 & -- & -- & -- & -- & -- & -- \\
\hline $\begin{array}{l}\text { Cadmium, in micrograms } \\
\text { per liter }\end{array}$ & 2007 & 4 & 1 & 4 & ${ }^{s}<0.04$ & -- & 4 & 4 & -- & 0.23 & 0 & 0.86 & 0 & L \\
\hline $\begin{array}{l}\text { Cadmium, in micrograms } \\
\text { per liter }\end{array}$ & 2008-2009 & 8 & 5 & 4 & ${ }^{s}<0.04$ & -- & 4 & 4 & -- & 0.23 & 0 & 0.86 & 0 & $\mathrm{~L}$ \\
\hline Copper, in micrograms per liter & 2007 & 4 & 0 & 3.1 & 7.3 & $04 / 11 / 07$ & 1.1 & 7.3 & -- & 4.6 & 1 & 6.4 & 1 & $\mathrm{H}$ \\
\hline Copper, in micrograms per liter & 2008-2009 & 8 & 4 & 0.27 & 0.86 & 06/09/09 & 4 & 0.83 & -- & 4.6 & 0 & 6.4 & 0 & $\mathrm{~L}$ \\
\hline Iron, in micrograms per liter & 2007 & 4 & 0 & 115 & 207 & $08 / 14 / 07$ & 52 & 207 & -- & 300 & 0 & -- & -- & M \\
\hline Iron, in micrograms per liter & 2008-2009 & 8 & 0 & 125 & 206 & $04 / 29 / 09$ & 77 & 197 & -- & 300 & 0 & -- & -- & M \\
\hline Lead, in micrograms per liter & 2007 & 4 & 0 & 0.27 & 0.35 & $01 / 17 / 07$ & 0.19 & 0.35 & -- & 1.06 & 0 & 27.1 & 0 & $\mathrm{~L}$ \\
\hline Lead, in micrograms per liter & 2008-2009 & 8 & 0 & 0.09 & 0.19 & $05 / 20 / 08$ & 0.06 & 0.16 & -- & 1.06 & 0 & 27.1 & 0 & $\mathrm{~L}$ \\
\hline $\begin{array}{l}\text { Manganese, in micrograms } \\
\text { per liter }\end{array}$ & 2007 & 4 & 0 & 7.1 & 13.6 & 04/11/07 & 6.5 & 13.6 & -- & 1,269 & 0 & 2,296 & 0 & $\mathrm{~L}$ \\
\hline $\begin{array}{l}\text { Manganese, in micrograms } \\
\text { per liter }\end{array}$ & 2008-2009 & 8 & 0 & 8.8 & 16.1 & $05 / 20 / 08$ & 4.6 & 14.7 & -- & 1,269 & 0 & 2,296 & 0 & $\mathrm{~L}$ \\
\hline Silver, in micrograms per liter & 2007 & 4 & 4 & 4 & ${ }^{s}<0.1$ & -- & 4 & 4 & -- & 0.02 & 0 & 0.52 & 0 & $\mathrm{~L}$ \\
\hline Silver, in micrograms per liter & 2008-2009 & 8 & 7 & ${ }^{4}$ & ${ }^{5}<0.1$ & -- & 4 & 4 & -- & 0.02 & 0 & 0.52 & 0 & $\mathrm{~L}$ \\
\hline Zinc, in micrograms per liter & 2007 & 4 & 0 & 7.8 & 13.5 & 04/11/07 & 2.2 & 13.5 & -- & 18.1 & 0 & 73.2 & 0 & M \\
\hline Zinc, in micrograms per liter & 2008-2009 & 8 & 0 & 1.8 & 3.3 & 06/09/09 & 1.2 & 3.2 & -- & 18.1 & 0 & 73.2 & 0 & $\mathrm{~L}$ \\
\hline $\begin{array}{l}\text { Suspended sediment, in mil- } \\
\text { ligrams per liter }\end{array}$ & 2007 & 4 & 0 & 4.5 & 8.0 & 06/05/07 & 1.0 & 8.0 & -- & -- & -- & -- & -- & -- \\
\hline $\begin{array}{l}\text { Suspended sediment, in mil- } \\
\text { ligrams per liter }\end{array}$ & 2008-2009 & 8 & 0 & 3.5 & 26.0 & 05/20/08 & 2.0 & 19.0 & -- & -- & -- & -- & -- & -- \\
\hline
\end{tabular}
ligrams per liter
${ }^{1}$ Censored values
${ }^{1}$ Ceometic ment

(a)

.

${ }^{4}$ Median values and percentiles were not tabled when they were calculated as zero.

${ }^{5}$ All values below laboratory reporting level (LRL), current LRL used for current period maximum, highest LRL used for historic period.

${ }^{6}$ Use geometric mean for comparison to standard. 


\section{East River below Cement Creek, Colorado}

Current Reason for Inclusion: Long-term monitoring. First station on East River downstream from the confluence with the Slate River.

General Station Information:

Location:

Station Type:

Period of Record:

Streamflow gaging:

Latitude: 384703

Longitude: 1065613
1.6 mi downstream from Cement Creek ( 8.5 mi southeast of Crested Butte).

USGS water quality and streamflow gaging

Water quality: October 1993-September 2009

October 1963-September 1972

October 1979-September 1981

October 1993-September 2009

Drainage area: $238 \mathrm{mi}^{2} \quad$ HUC: 14020001

Stream segment: 5

USGS Data Summary: Water Years 2008-2009:

General Chemistry:

$\mathrm{pH}$ : Low concern

Dissolved oxygen: Low concern

Temperature: Low concern

Nutrients:

Low concern

E. coli:

Low concern

Trace Elements/metals:

No data

Other constituents of concern: $\quad$ None for constituents tested.

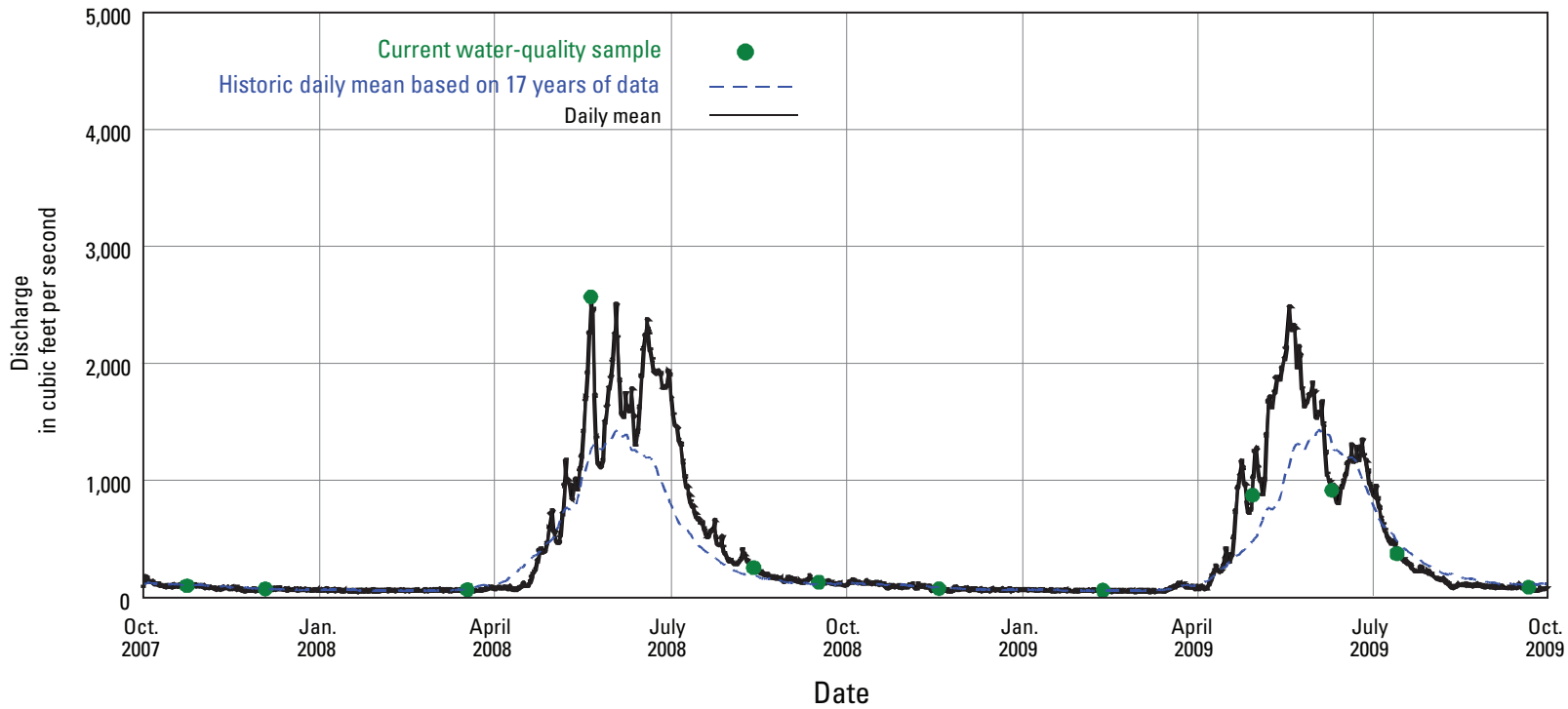

Figure 12. Daily mean discharge and time distribution of water-quality samples for East River below Cement Creek, Colorado. 

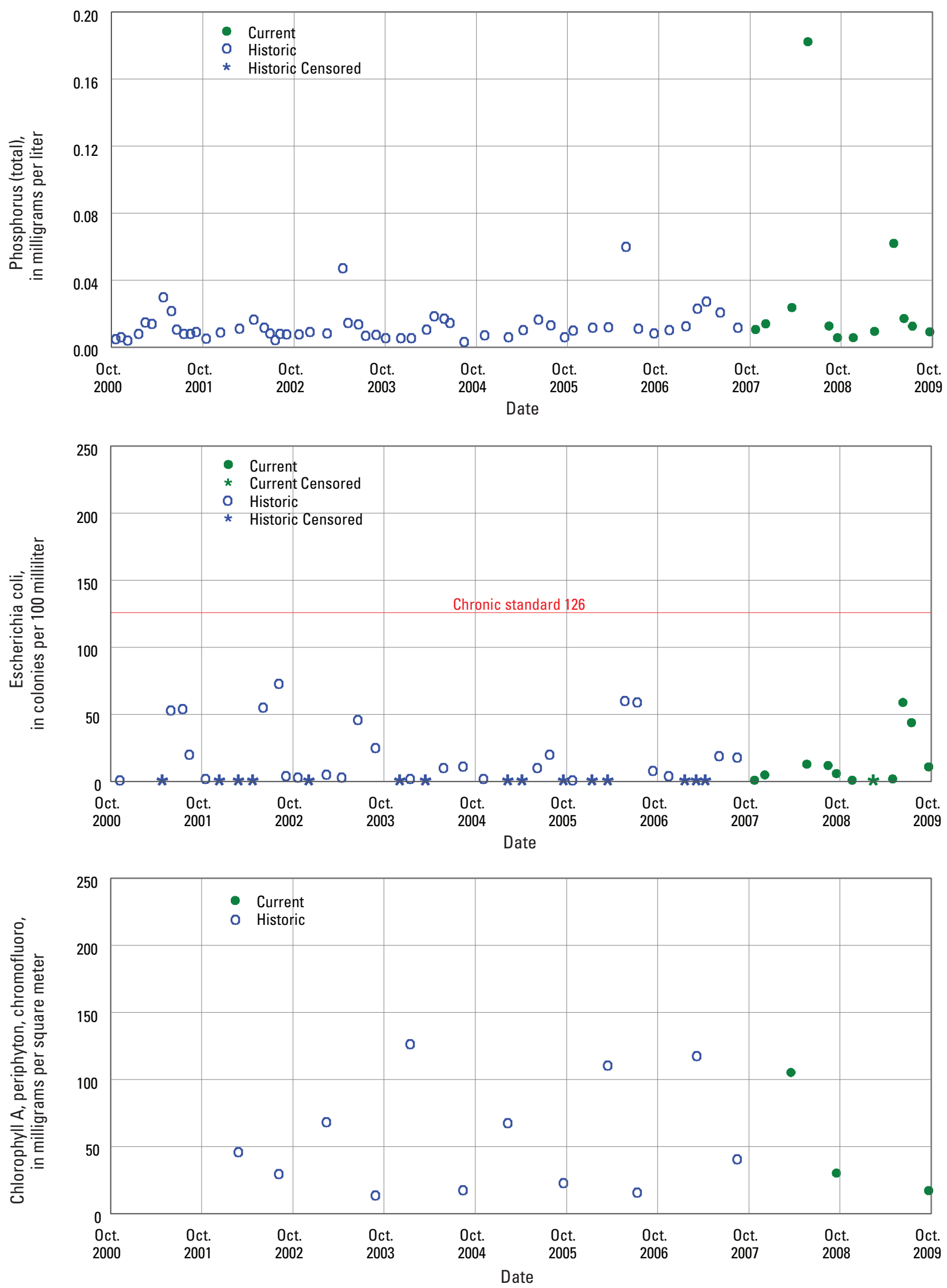

Figure 13. Time-series plots of selected water-quality constituents for East River below Cement Creek, Colorado. 
Table 9. Summary of measured constituents and properties for East River below Cement Creek, Colorado, station 09112200.

[--, no data or not applicable; L, low; M, medium; H, high; E. coli, Escherichia coli; $\mathrm{mg} / \mathrm{m}^{2}$, milligrams per square meter; USEPA, U.S. Environmental Protection Agency]

\begin{tabular}{|c|c|c|c|c|c|c|c|c|c|c|c|c|c|c|}
\hline $\begin{array}{l}\text { Property } \\
\text { or } \\
\text { constituent }\end{array}$ & $\begin{array}{l}\text { Period } \\
\text { (water } \\
\text { years) }\end{array}$ & $\begin{array}{c}\text { Number } \\
\text { of } \\
\text { samples }\end{array}$ & $\begin{array}{c}\text { Number } \\
\text { of } \\
\text { censored } \\
\text { values }\end{array}$ & Median' & Maximum & $\begin{array}{c}\text { Date } \\
\text { of } \\
\text { Maximum }\end{array}$ & $\begin{array}{c}\text { 15th } \\
\text { percentile }\end{array}$ & $\begin{array}{c}\text { 85th } \\
\text { percentile }\end{array}$ & $\begin{array}{c}\text { Geometric } \\
\text { Mean }^{2}\end{array}$ & $\begin{array}{l}\text { Standard } \\
\text { or } \\
\text { Chronic } \\
\text { standard }^{3} \\
\end{array}$ & $\begin{array}{l}\text { Number of } \\
\text { exceedences } \\
\text { Standard or } \\
\text { Chronic } \\
\end{array}$ & $\begin{array}{l}\text { Acute } \\
\text { standard }\end{array}$ & $\begin{array}{c}\begin{array}{c}\text { Number } \\
\text { of } \\
\text { exceedences } \\
\text { Acute }\end{array} \\
\end{array}$ & $\begin{array}{c}\text { Level } \\
\text { of } \\
\text { concern }\end{array}$ \\
\hline $\begin{array}{l}\text { Instantaneous discharge, in cubic feet per } \\
\text { second }\end{array}$ & 1994-2007 & 156 & 0 & 107 & 3,030 & $06 / 16 / 95$ & 63 & 880 & -- & -- & -- & -- & -- & -- \\
\hline $\begin{array}{l}\text { Instantaneous discharge, in cubic feet per } \\
\text { second }\end{array}$ & 2008-2009 & 14 & 0 & 115 & 2,570 & $05 / 21 / 08$ & 65 & 906 & -- & -- & -- & -- & -- & -- \\
\hline Dissolved oxygen, in milligrams per liter & 1994-2007 & 146 & 0 & 9.4 & 12.7 & $03 / 25 / 94$ & 7.9 & 10.9 & -- & 6.0 & 0 & -- & -- & $\mathrm{L}$ \\
\hline Dissolved oxygen, in milligrams per liter & 2008-2009 & 11 & 0 & 9.9 & 12.1 & 03/18/08 & 8.2 & 10.8 & -- & 6.0 & 0 & -- & -- & $\mathrm{L}$ \\
\hline $\mathrm{pH}$, in standard units & 1994-2007 & 147 & 0 & 8.3 & 9.3 & $403 / 06 / 07$ & 8.0 & 8.6 & -- & 6.5 & 3 & 9.0 & -- & $\mathrm{L}$ \\
\hline $\mathrm{pH}$, in standard units & 2008-2009 & 12 & 0 & 8.4 & 8.9 & 03/18/08 & 7.8 & 8.9 & -- & 6.5 & 0 & 9.0 & -- & $\mathrm{L}$ \\
\hline $\begin{array}{l}\text { Specific conductance, in microsiemens per } \\
\text { centimeter }\end{array}$ & 1994-2007 & 150 & 0 & 279 & 335 & $09 / 03 / 02$ & 178 & 314 & -- & -- & -- & -- & -- & -- \\
\hline $\begin{array}{l}\text { Specific conductance, in microsiemens per } \\
\text { centimeter }\end{array}$ & 2008-2009 & 12 & 0 & 293 & 322 & 09/17/08 & 188 & 316 & -- & -- & -- & -- & -- & -- \\
\hline Temperature, degrees Celsius & $1994-2007$ & 150 & 0 & 5.6 & 17.2 & $08 / 05 / 98$ & 1.1 & 11.9 & -- & 17.0 & 1 & -- & -- & $\mathrm{L}$ \\
\hline Temperature, degrees Celsius & 2008-2009 & 12 & 0 & 6.5 & 15.4 & 07/14/09 & 2.9 & 14.4 & -- & 17.0 & 0 & -- & -- & $\mathrm{L}$ \\
\hline $\begin{array}{l}\text { Ammonia plus organic nitrogen, in mil- } \\
\text { ligrams per liter }\end{array}$ & 1995-2007 & 140 & 46 & 0.09 & 0.40 & $04 / 23 / 97$ & 5 & 0.20 & -- & -- & -- & -- & -- & -- \\
\hline $\begin{array}{l}\text { Ammonia plus organic nitrogen, in mil- } \\
\text { ligrams per liter }\end{array}$ & 2008-2009 & 12 & 1 & 0.10 & 0.54 & $05 / 21 / 08$ & 0.07 & 0.29 & -- & -- & -- & -- & -- & -- \\
\hline Ammonia nitrogen, in milligrams per liter & 1994-2007 & 146 & 73 & 0.001 & 0.118 & $03 / 19 / 98$ & 5 & 0.024 & -- & 1.56 & 0 & 3.39 & 0 & $\mathrm{~L}$ \\
\hline Ammonia nitrogen, in milligrams per liter & 2008-2009 & 12 & 10 & 5 & 0.018 & $08 / 14 / 08$ & 5 & 0.011 & -- & 1.71 & 0 & 4.13 & 0 & $\mathrm{~L}$ \\
\hline $\begin{array}{l}\text { Nitrite plus nitrate nitrogen, in milligrams } \\
\text { per liter }\end{array}$ & 1994-2007 & 146 & 6 & 0.085 & 0.322 & $05 / 06 / 03$ & 0.041 & 0.160 & -- & 10.0 & 0 & -- & -- & $\mathrm{L}$ \\
\hline $\begin{array}{l}\text { Nitrite plus nitrate nitrogen, in milligrams } \\
\text { per liter }\end{array}$ & 2008-2009 & 12 & 0 & 0.064 & 0.133 & $12 / 04 / 07$ & 0.035 & 0.131 & -- & 10.0 & 0 & -- & -- & $\mathrm{L}$ \\
\hline Nitrite nitrogen, in milligrams per liter & 1994-2007 & 146 & 83 & 5 & 0.020 & 03/27/96 & 5 & 0.002 & -- & 0.05 & 0 & -- & -- & $\mathrm{L}$ \\
\hline Nitrite nitrogen, in milligrams per liter & 2008-2009 & 12 & 6 & 0.001 & 0.002 & 03/18/08 & 5 & 0.002 & -- & 0.05 & 0 & -- & -- & $\mathrm{L}$ \\
\hline Orthophosphate, in milligrams per liter & 1994-2007 & 146 & 99 & 5 & 0.042 & $02 / 19 / 98$ & 5 & 0.008 & -- & -- & -- & -- & -- & -- \\
\hline Orthophosphate, in milligrams per liter & $2008-2009$ & 12 & 2 & 0.005 & 0.008 & 07/14/09 & 5 & 0.007 & -- & -- & -- & -- & -- & -- \\
\hline Phosphorus (total), in milligrams per liter & 1995-2007 & 139 & 26 & 0.010 & 0.090 & $06 / 16 / 95$ & 5 & 0.031 & -- & -- & -- & -- & -- & -- \\
\hline Phosphorus (total), in milligrams per liter & 2008-2009 & 12 & 0 & 0.013 & 0.182 & $05 / 21 / 08$ & 0.006 & 0.068 & -- & -- & -- & -- & -- & -- \\
\hline E. coli, in colonies per 100 milliliter & 1994-2007 & 54 & 18 & 3 & 82 & $06 / 01 / 94$ & 1 & 6 & 4.6 & 126 & 0 & -- & -- & $\mathrm{L}$ \\
\hline E. coli, in colonies per 100 milliliter & 2008-2009 & 11 & 1 & 6 & 59 & 06/10/09 & 1 & 6 & -- & 126 & 0 & -- & -- & $\mathrm{L}$ \\
\hline $\begin{array}{l}\text { Biomass periphyton, ashfree drymass, in } \\
\text { grams per square meter }\end{array}$ & $1997-2007$ & 16 & 0 & 26.3 & 64.4 & $01 / 14 / 04$ & 4.2 & 53.3 & -- & -- & -- & -- & -- & -- \\
\hline
\end{tabular}


Table 9. Summary of measured constituents and properties for East River below Cement Creek, Colorado, station 09112200.—Continued

[--, no data or not applicable; L, low; M, medium; H, high; E. coli, Escherichia coli; mg/m², milligrams per square meter; USEPA, U.S. Environmental Protection Agency]

\begin{tabular}{|c|c|c|c|c|c|c|c|c|c|c|c|c|c|c|}
\hline $\begin{array}{c}\text { Property } \\
\text { or } \\
\text { constituent }\end{array}$ & $\begin{array}{l}\text { Period } \\
\text { (water } \\
\text { years) }\end{array}$ & $\begin{array}{c}\text { Number } \\
\text { of } \\
\text { samples }\end{array}$ & $\begin{array}{c}\text { Number } \\
\text { of } \\
\text { censored } \\
\text { values }\end{array}$ & Median ${ }^{1}$ & Maximum & $\begin{array}{c}\text { Date } \\
\text { of } \\
\text { Maximum }\end{array}$ & $\begin{array}{c}\text { 15th } \\
\text { percentile }\end{array}$ & $\begin{array}{c}\text { 85th } \\
\text { percentile }\end{array}$ & $\begin{array}{c}\text { Geometric } \\
\text { Mean }^{2}\end{array}$ & $\begin{array}{c}\text { Standard } \\
\text { or } \\
\text { Chronic } \\
\text { standard }\end{array}$ & $\begin{array}{c}\text { Number of } \\
\text { exceedences } \\
\text { Standard or } \\
\text { Chronic }\end{array}$ & $\begin{array}{l}\text { Acute } \\
\text { standard }\end{array}$ & $\begin{array}{c}\text { Number } \\
\text { of } \\
\text { exceedences } \\
\text { Acute }\end{array}$ & $\begin{array}{c}\text { Level } \\
\text { of } \\
\text { concern }\end{array}$ \\
\hline $\begin{array}{l}\text { Biomass periphyton, ashfree drymass, in } \\
\text { grams per square meter }\end{array}$ & 2008-2009 & 3 & 0 & 16.6 & 26.6 & $03 / 18 / 08$ & 9.1 & 26.6 & -- & -- & -- & -- & -- & -- \\
\hline $\begin{array}{l}\text { Periphyton, biomass, ash weight, in grams } \\
\text { per square meter }\end{array}$ & 1997-2007 & 16 & 0 & 558 & 1,061 & $08 / 27 / 03$ & 77.4 & 845 & -- & -- & -- & -- & -- & -- \\
\hline $\begin{array}{l}\text { Periphyton, biomass, ash weight, in grams } \\
\text { per square meter }\end{array}$ & 2008-2009 & 3 & 0 & 387 & 463 & 03/18/08 & 293 & 463 & -- & -- & -- & -- & -- & -- \\
\hline $\begin{array}{l}\text { Periphyton, biomass, dry weight, in grams } \\
\text { per square meter }\end{array}$ & 1997-2007 & 16 & 0 & 589 & 1,087 & $08 / 27 / 03$ & 81.6 & 900 & -- & -- & -- & -- & -- & -- \\
\hline $\begin{array}{l}\text { Periphyton, biomass, dry weight, in grams } \\
\text { per square meter }\end{array}$ & 2008-2009 & 3 & 0 & 403 & 490 & 03/18/08 & 302 & 490 & -- & -- & -- & -- & -- & -- \\
\hline $\begin{array}{l}\text { Chlorophyll a, periphyton, chromofluoro, } \\
\text { in } \mathrm{mg} / \mathrm{m}^{2}\end{array}$ & 1997-2007 & 16 & 0 & 35.0 & 126 & $01 / 14 / 04$ & 5.98 & 114 & -- & -- & -- & -- & -- & -- \\
\hline $\begin{array}{l}\text { Chlorophyll a, periphyton, chromofluoro, } \\
\text { in } \mathrm{mg} / \mathrm{m}^{2}\end{array}$ & 2008-2009 & 3 & 0 & 30.3 & 105 & $03 / 18 / 08$ & 17.3 & 105 & -- & -- & -- & -- & -- & -- \\
\hline Pheophytin a, periphyton, in $\mathrm{mg} / \mathrm{m}^{2}$ & 2002-2007 & 12 & 0 & 19.8 & 70.0 & $01 / 14 / 04$ & 4.44 & 56.2 & -- & -- & -- & -- & -- & -- \\
\hline Pheophytin a, periphyton, in $\mathrm{mg} / \mathrm{m}^{2}$ & 2008-2009 & 3 & 0 & 11.0 & 30.5 & $03 / 18 / 08$ & 6.71 & 30.5 & -- & -- & -- & -- & -- & -- \\
\hline
\end{tabular}

${ }^{1}$ Censored values were replaced with 0 to compute median and 85 th percentiles (coliform censored values replaced with 1) see definitions section.

'Geometric mean listed for E. coli. Geometric mean computed for combined period of record (1994-2009).

${ }^{3}$ Colorado Department of Public Health and Environment, classification and numeric standards for Gunnison and Lower Dolores River Basins (2010a); and USEPA National Recommended Water Quality Criteria (2009).

${ }^{4}$ Multiple dates for maximum.

${ }^{5}$ Median values and percentiles were not tabled when they were calculated as zero.

${ }^{6}$ Use geometric mean for comparison to standard. 
Table 10. Summary of seasonal Kendall trend tests for East River below Cement Creek, Colorado, station 09112200.

[E.coli, Escherichia coli]

\begin{tabular}{lcccc}
\hline \multicolumn{1}{c}{ Property or constituent } & $\begin{array}{c}\text { Period } \\
\text { (water years) }\end{array}$ & Kendall tau $(\tau)$ & p-Value & Trend direction \\
\hline Specific conductance, in microsiemens per centimeter & $1995-2009$ & 0.169 & 0.083 & None \\
pH, in standard units & $1995-2009$ & 0.181 & 0.062 & None \\
Ammonia plus organic nitrogen, in milligrams per liter & $1999-2009$ & 0.118 & 0.330 & None \\
Nitrite plus nitrate nitrogen, in milligrams per liter & $1999-2009$ & -0.068 & 0.586 & None \\
Phosphorus (total), in milligrams per liter & $1999-2009$ & 0.259 & 0.029 & Up \\
E. coli, in colonies per 100 milliliter & $2001-2009$ & -0.116 & 0.413 & None \\
\hline
\end{tabular}




\section{East River at Almont, Colorado}

Current Reason for Inclusion: Characterizes water quality at the mouth of the East River. Long-term monitoring.

\section{General Station Information:}

Location:

Station Type:

Period of Record:

Streamflow gaging:

Latitude: 383952

Longitude: 1065051
$400 \mathrm{ft}$ upstream from confluence with Taylor River.

USGS water quality and streamflow gaging

Water quality: October 1990-September 2009

October 1934-September 2009

Drainage area: $289 \mathrm{mi}^{2}$

HUC: 14020001

USGS Data Summary: Water Years 2008-2009:

General Chemistry

$\mathrm{pH}$ : Low concern

Dissolved oxygen: Low concern

Temperature: Low concern

Nutrients:

Low concern

E. coli:

Low concern

Trace Elements/metals:

No data

Other constituents of concern: None for constituents tested.

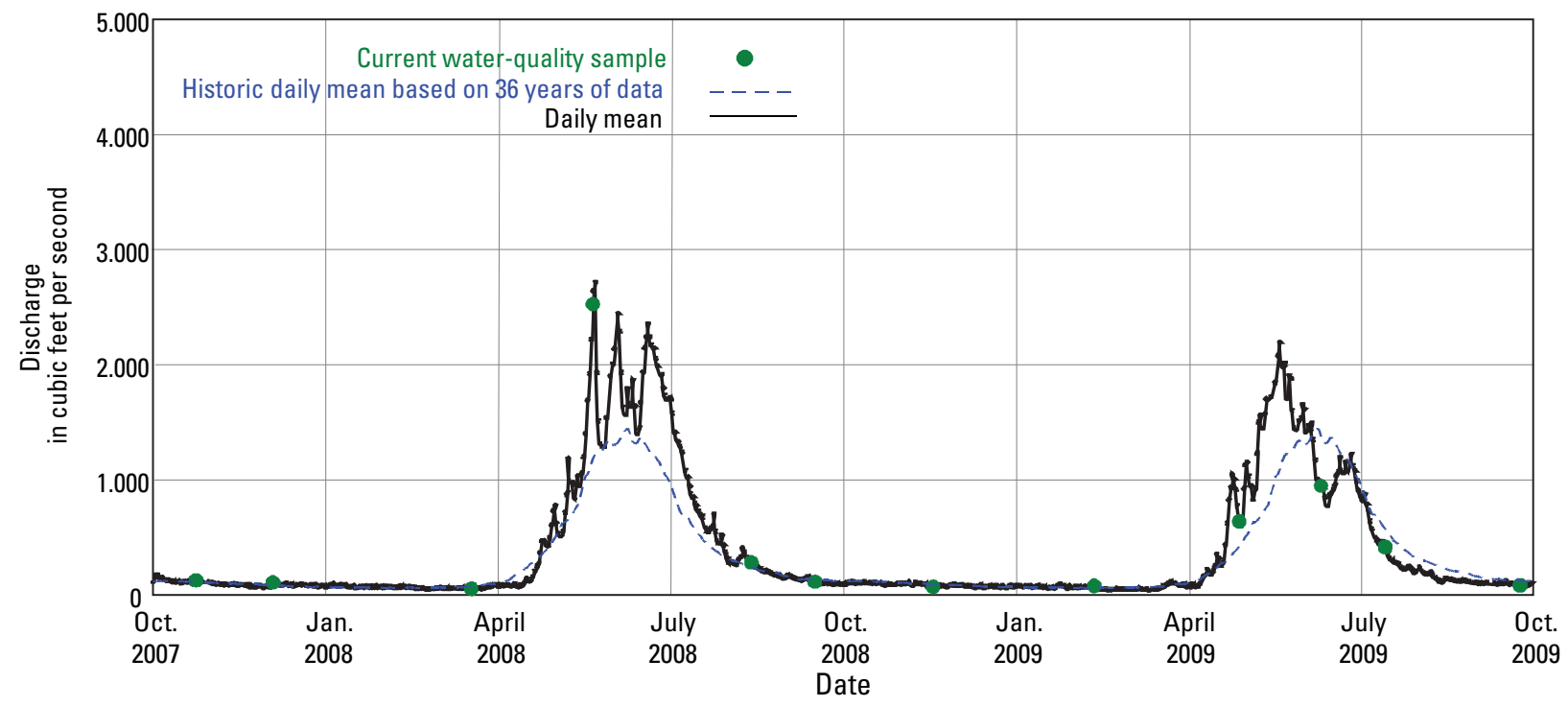

Figure 14. Daily mean discharge and time distribution of water-quality samples for East River at Almont, Colorado. 

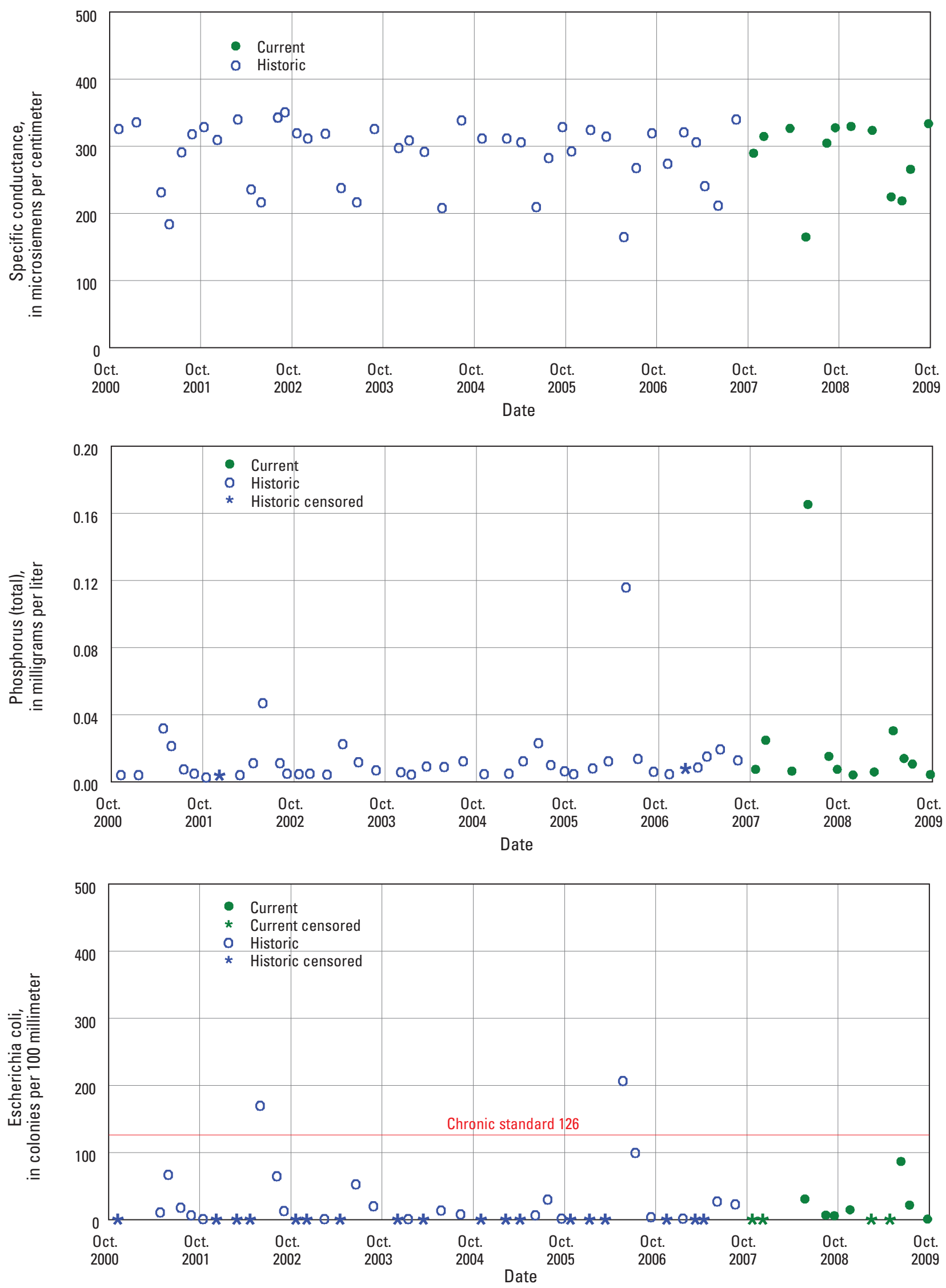

Figure 15. Time-series plots of selected water-quality constituents for East River at Almont, Colorado. 
Table 11. Summary of measured constituents and properties for East River at Almont, Colorado, station 09112500.

[--, no data or not applicable; L, low; M, medium; H, high; LRL, Lab Reporting Level; E. coli, Escherichia coli; mg/m², milligrams per square meter; USEPA, U.S. Environmental Protection Agency]

\begin{tabular}{|c|c|c|c|c|c|c|c|c|c|c|c|c|c|c|}
\hline $\begin{array}{l}\text { Property } \\
\text { or } \\
\text { constituent }\end{array}$ & $\begin{array}{l}\text { Period } \\
\text { (water } \\
\text { years) }\end{array}$ & $\begin{array}{c}\text { Number } \\
\text { of } \\
\text { samples }\end{array}$ & $\begin{array}{c}\begin{array}{c}\text { Number } \\
\text { of } \\
\text { censored } \\
\text { values }\end{array} \\
\end{array}$ & Median' & Maximum & $\begin{array}{c}\text { Date } \\
\text { of } \\
\text { Maximum }\end{array}$ & $\begin{array}{c}\text { 15th } \\
\text { percentile }\end{array}$ & $\begin{array}{c}\text { 85th } \\
\text { percentile }\end{array}$ & $\begin{array}{c}\text { Geometric } \\
\text { Mean }^{2}\end{array}$ & $\begin{array}{l}\text { Standard } \\
\text { or } \\
\text { Chronic } \\
\text { standard }^{3}\end{array}$ & $\begin{array}{c}\text { Number of } \\
\text { exceedences } \\
\text { Standard or } \\
\text { Chronic } \\
\end{array}$ & $\begin{array}{l}\text { Acute } \\
\text { standard }\end{array}$ & $\begin{array}{c}\text { Number of } \\
\text { exceedences } \\
\text { Acute }\end{array}$ & $\begin{array}{c}\text { Level } \\
\text { of } \\
\text { concern }\end{array}$ \\
\hline $\begin{array}{l}\text { Instantaneous discharge, in cubic feet per } \\
\text { second }\end{array}$ & 1994-2007 & 93 & 0 & 130 & 2,710 & $07 / 13 / 95$ & 74 & 820 & -- & -- & -- & -- & -- & -- \\
\hline $\begin{array}{l}\text { Instantaneous discharge, in cubic feet per } \\
\text { second }\end{array}$ & $2008-2009$ & 12 & 0 & 120 & 2,525 & $05 / 21 / 08$ & 69 & 1,026 & -- & -- & -- & -- & -- & -- \\
\hline Dissolved oxygen, in milligrams per liter & 1994-2007 & 87 & 0 & 9.2 & 12.8 & $411 / 17 / 93$ & 8.0 & 11.0 & -- & 6.0 & 0 & -- & -- & $\mathrm{L}$ \\
\hline Dissolved oxygen, in milligrams per liter & 2008-2009 & 12 & 0 & 9.7 & 11.4 & $12 / 04 / 07$ & 8.0 & 11.4 & -- & 6.0 & 0 & -- & -- & $\mathrm{L}$ \\
\hline $\mathrm{pH}$, in standard units & 1994-2007 & 88 & 0 & 8.4 & 8.9 & $411 / 17 / 93$ & 8.2 & 8.7 & -- & 6.5 & 0 & 9.0 & -- & $\mathrm{L}$ \\
\hline $\mathrm{pH}$, in standard units & 2008-2009 & 12 & 0 & 8.1 & 8.7 & 08/13/08 & 8.0 & 8.5 & -- & 6.5 & 0 & 9.0 & -- & $\mathrm{L}$ \\
\hline $\begin{array}{l}\text { Specific conductance, in microsiemens per } \\
\text { centimeter }\end{array}$ & 1994-2007 & 88 & 0 & 298 & 351 & 09/04/02 & 210 & 328 & -- & -- & -- & -- & -- & -- \\
\hline $\begin{array}{l}\text { Specific conductance, in microsiemens per } \\
\text { centimeter }\end{array}$ & 2008-2009 & 12 & 0 & 310 & 334 & 09/24/09 & 216 & 330 & -- & -- & -- & -- & -- & -- \\
\hline Temperature, degrees Celsius & 1994-2007 & 88 & 0 & 7.6 & 17.0 & 07/13/99 & 0.6 & 13.5 & -- & 17.0 & 0 & -- & -- & $\mathrm{L}$ \\
\hline Temperature, degrees Celsius & 2008-2009 & 12 & 0 & 6.1 & 16.5 & 07/14/09 & 0.3 & 15.6 & -- & 17.0 & 0 & -- & -- & $\mathrm{L}$ \\
\hline $\begin{array}{l}\text { Ammonia plus organic nitrogen, in mil- } \\
\text { ligrams per liter }\end{array}$ & $1995-2007$ & 82 & 27 & 0.09 & 0.44 & 05/23/06 & 5 & 0.16 & -- & -- & -- & -- & -- & -- \\
\hline $\begin{array}{l}\text { Ammonia plus organic nitrogen, in mil- } \\
\text { ligrams per liter }\end{array}$ & 2008-2009 & 12 & 2 & 0.12 & 0.51 & $05 / 21 / 08$ & 5 & 0.22 & -- & -- & -- & -- & -- & -- \\
\hline Ammonia nitrogen, in milligrams per liter & 1994-2007 & 88 & 52 & 5 & 0.040 & $411 / 20 / 96$ & 5 & 0.020 & -- & 1.41 & 0 & 3.02 & 0 & $\mathrm{~L}$ \\
\hline Ammonia nitrogen, in milligrams per liter & 2008-2009 & 12 & 9 & 5 & 0.033 & 08/13/08 & 5 & 0.015 & -- & 1.89 & 0 & 4.35 & 0 & $\mathrm{~L}$ \\
\hline $\begin{array}{l}\text { Nitrite plus nitrate nitrogen, in milligrams } \\
\text { per liter }\end{array}$ & 1994-2007 & 87 & 9 & 0.070 & 0.283 & $04 / 16 / 03$ & 0.011 & 0.110 & -- & 10.0 & 0 & -- & -- & $\mathrm{L}$ \\
\hline $\begin{array}{l}\text { Nitrite plus nitrate nitrogen, in milligrams } \\
\text { per liter }\end{array}$ & 2008-2009 & 12 & 2 & 0.043 & 0.130 & $05 / 21 / 08$ & 5 & 0.120 & -- & 10.0 & 0 & -- & -- & $\mathrm{L}$ \\
\hline Nitrite nitrogen, in milligrams per liter & 1994-2007 & 88 & 49 & 5 & 0.030 & $11 / 20 / 96$ & 5 & 0.002 & -- & 0.05 & 0 & -- & -- & $\mathrm{L}$ \\
\hline Nitrite nitrogen, in milligrams per liter & 2008-2009 & 12 & 8 & 5 & 0.002 & 05/21/08 & 5 & 0.001 & -- & 0.05 & 0 & -- & -- & $\mathrm{L}$ \\
\hline Orthophosphate, in milligrams per liter & 1994-2007 & 87 & 68 & 5 & 0.020 & $06 / 03 / 98$ & 5 & 0.003 & -- & -- & -- & -- & -- & -- \\
\hline Orthophosphate, in milligrams per liter & $2008-2009$ & 12 & 5 & 0.004 & 0.007 & 04/28/09 & 5 & 0.006 & -- & -- & -- & -- & -- & -- \\
\hline Phosphorus (total), in milligrams per liter & $1995-2007$ & 82 & 19 & 0.007 & 0.116 & 05/23/06 & 5 & 0.021 & -- & -- & -- & -- & -- & -- \\
\hline Phosphorus (total), in milligrams per liter & 2008-2009 & 12 & 0 & 0.009 & 0.166 & $05 / 21 / 08$ & 0.004 & 0.037 & -- & -- & -- & -- & -- & -- \\
\hline E. coli, in colonies per 100 milliliter & 1994-2007 & 53 & 21 & 2 & 300 & $10 / 26 / 95$ & 1 & 6 & 4.7 & 126 & 3 & -- & -- & $\mathrm{L}$ \\
\hline E. coli, in colonies per 100 milliliter & 2008-2009 & 11 & 4 & 6 & 87 & 06/10/09 & 1 & 6 & -- & 126 & 0 & -- & -- & $\mathrm{L}$ \\
\hline $\begin{array}{l}\text { Biomass periphyton, ashfree drymass, in } \\
\text { grams per square meter }\end{array}$ & 1998-2007 & 8 & 1 & 18.7 & 59.4 & 03/18/98 & 1.1 & 48.7 & -- & -- & -- & -- & -- & -- \\
\hline $\begin{array}{l}\text { Biomass periphyton, ashfree drymass, in } \\
\text { grams per square meter }\end{array}$ & 2008-2009 & 2 & 1 & 6.0 & 12.0 & 09/24/09 & 5 & 12.0 & -- & -- & -- & -- & -- & -- \\
\hline $\begin{array}{l}\text { Periphyton, biomass, ash weight, in grams } \\
\text { per square meter }\end{array}$ & 1998-2007 & 8 & 0 & 475 & 977 & $08 / 27 / 03$ & 100 & 949 & -- & -- & -- & -- & -- & -- \\
\hline
\end{tabular}


Table 11. Summary of measured constituents and properties for East River at Almont, Colorado, station 09112500.—Continued

[--, no data or not applicable; L, low; M, medium; H, high; LRL, Lab Reporting Level; E. coli, Escherichia coli; mg/m² milligrams per square meter; USEPA, U.S. Environmental Protection Agency]

\begin{tabular}{|c|c|c|c|c|c|c|c|c|c|c|c|c|c|c|}
\hline $\begin{array}{c}\text { Property } \\
\text { or } \\
\text { constituent }\end{array}$ & $\begin{array}{l}\text { Period } \\
\text { (water } \\
\text { years) }\end{array}$ & $\begin{array}{c}\text { Number } \\
\text { of } \\
\text { samples }\end{array}$ & $\begin{array}{c}\text { Number } \\
\text { of } \\
\text { censored } \\
\text { values }\end{array}$ & Median ${ }^{1}$ & Maximum & $\begin{array}{c}\text { Date } \\
\text { of } \\
\text { Maximum }\end{array}$ & $\begin{array}{c}\text { 15th } \\
\text { percentile }\end{array}$ & $\begin{array}{c}\text { 85th } \\
\text { percentile }\end{array}$ & $\begin{array}{c}\text { Geometric } \\
\text { Mean }^{2}\end{array}$ & $\begin{array}{c}\text { Standard } \\
\text { or } \\
\text { Chronic } \\
\text { standard }^{3}\end{array}$ & $\begin{array}{l}\text { Number of } \\
\text { exceedences } \\
\text { Standard or } \\
\text { Chronic }\end{array}$ & $\begin{array}{l}\text { Acute } \\
\text { standard }\end{array}$ & $\begin{array}{l}\text { Number of } \\
\text { exceedences } \\
\text { Acute }\end{array}$ & $\begin{array}{c}\text { Level } \\
\text { of } \\
\text { concern }\end{array}$ \\
\hline $\begin{array}{l}\text { Periphyton, biomass, ash weight, in grams } \\
\text { per square meter }\end{array}$ & $2008-2009$ & 2 & 0 & 337 & 404 & 09/24/09 & 270 & 404 & -- & -- & -- & -- & -- & -- \\
\hline $\begin{array}{l}\text { Periphyton, biomass, dry weight, in grams } \\
\text { per square meter }\end{array}$ & 1998-2007 & 8 & 0 & 493 & 994 & $08 / 27 / 03$ & 123 & 970 & -- & -- & -- & -- & -- & -- \\
\hline $\begin{array}{l}\text { Periphyton, biomass, dry weight, in grams } \\
\text { per square meter }\end{array}$ & $2008-2009$ & 2 & 0 & 346 & 416 & 09/24/09 & 276 & 416 & -- & -- & -- & -- & -- & -- \\
\hline $\begin{array}{l}\text { Chlorophyll a, periphyton, chromofluoro, } \\
\text { in } \mathrm{mg} / \mathrm{m}^{2}\end{array}$ & 1998-2007 & 9 & 0 & 14.8 & 40.6 & 03/18/98 & 5.82 & 29.5 & -- & -- & -- & -- & -- & -- \\
\hline $\begin{array}{l}\text { Chlorophyll a, periphyton, chromofluoro, } \\
\text { in } \mathrm{mg} / \mathrm{m}^{2}\end{array}$ & 2008-2009 & 2 & 0 & 15.9 & 18.1 & 09/24/09 & 13.6 & 18.1 & -- & -- & -- & -- & -- & -- \\
\hline Pheophytin a, periphyton, in $\mathrm{mg} / \mathrm{m}^{2}$ & 2001-2007 & 7 & 0 & 6.81 & 9.1 & 07/17/01 & 4.6 & 9.0 & -- & -- & -- & -- & -- & -- \\
\hline Pheophytin a, periphyton, in $\mathrm{mg} / \mathrm{m}^{2}$ & 2008-2009 & 2 & 0 & 4.72 & 6.8 & 09/24/09 & 2.7 & 6.8 & -- & -- & .- & -- & .- & _- \\
\hline
\end{tabular}

${ }^{1}$ Censored values were replaced with 0 to compute median and 85 th percentiles (coliform censored values replaced with 1) see definitions section.

${ }^{2}$ Geometric mean listed for E. coli. Geometric mean computed for combined period of record (1994-2009).

${ }^{3}$ Colorado Department of Public Health and Environment, classification and numeric standards for Gunnison and Lower Dolores River Basins (2010a); and USEPA National Recommended Water Quality Criteria (2009). ${ }^{4}$ Multiple dates for maximum.

${ }^{5}$ Median values and percentiles were not tabled when they were calculated as zero.

${ }^{6}$ Use geometric mean for comparison to standard. 
Table 12. Summary of seasonal Kendall trend tests for East River at Almont, Colorado, station 09112500.

[E.coli, Escherichia coli]

\begin{tabular}{lcccc}
\hline \multicolumn{1}{c}{ Property or constituent } & $\begin{array}{c}\text { Period } \\
\text { (water years) }\end{array}$ & Kendall tau $(\tau)$ & p-Value & Trend direction \\
\hline Specific conductance, in microsiemens per centimeter & $1995-2009$ & 0.324 & $<0.001$ & Up \\
pH, in standard units & $1995-2009$ & -0.036 & 0.728 & None \\
Ammonia plus organic nitrogen, in milligrams per liter & $1999-2009$ & 0.132 & 1.000 & None \\
Nitrite plus nitrate nitrogen, in milligrams per liter & $2000-2009$ & 0.000 & 0.007 & None \\
Phosphorus (total), in milligrams per liter & $2000-2009$ & 0.339 & 0.790 & Np \\
E. coli, in colonies per 100 milliliter & $2001-2009$ & 0.039 & None \\
\hline
\end{tabular}




\section{Ohio Creek above Mouth, near Gunnison, Colorado}

Current Reason for Inclusion: This station characterizes the contributions of the Ohio Creek Basin, which includes development and historic mining. Long-term monitoring.

General Station Information:

Location:

County Road 48 bridge, 1.1 mi upstream from the confluence with the Gunnison River, and 3.1 mi north of Gunnison. The gage is on the left bank.

Station Type: $\quad$ USGS water quality and streamflow gaging

Period of Record: $\quad$ Water quality: October 1996-September 2009

Data prior to September 1998 published as station 383516106555000

Streamflow gaging: December 1998-September 2009

Latitude: 383516

Longitude: 1065551

Drainage area: $161 \mathrm{mi}^{2}$

HUC: 14020002

Stream segment: 16

USGS Data Summary: Water Years 2008-2009:

General Chemistry:

$\mathrm{pH}$ : Low concern

Dissolved oxygen: Low concern

Water Temperature: Low concern

Nutrients:

Low concern

E. coli:

Low concern

Trace Elements/metals:

No data

Other constituents of concern: $\quad$ None for constituents tested.

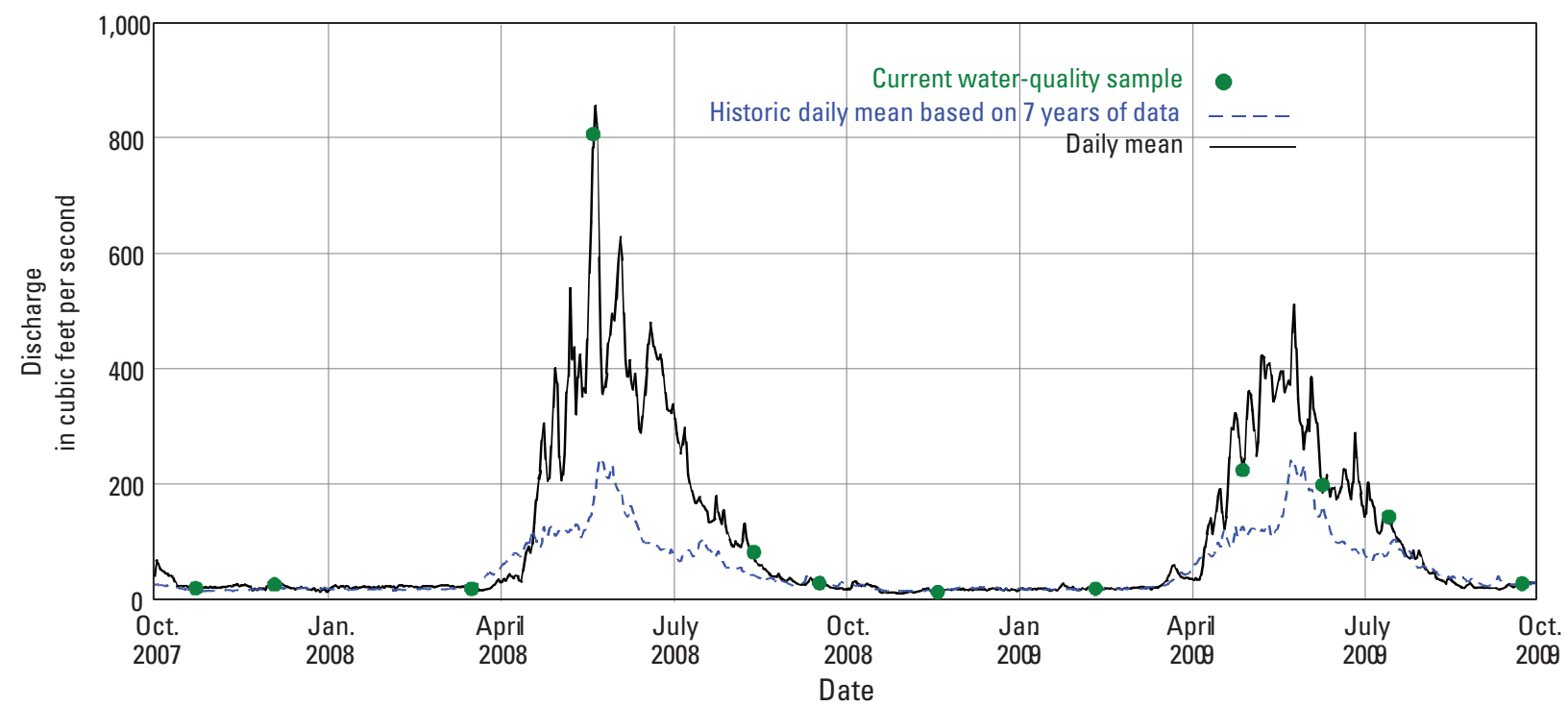

Figure 16. Daily mean discharge and time distribution of water-quality samples for Ohio Creek above mouth near Gunnison, Colorado. 

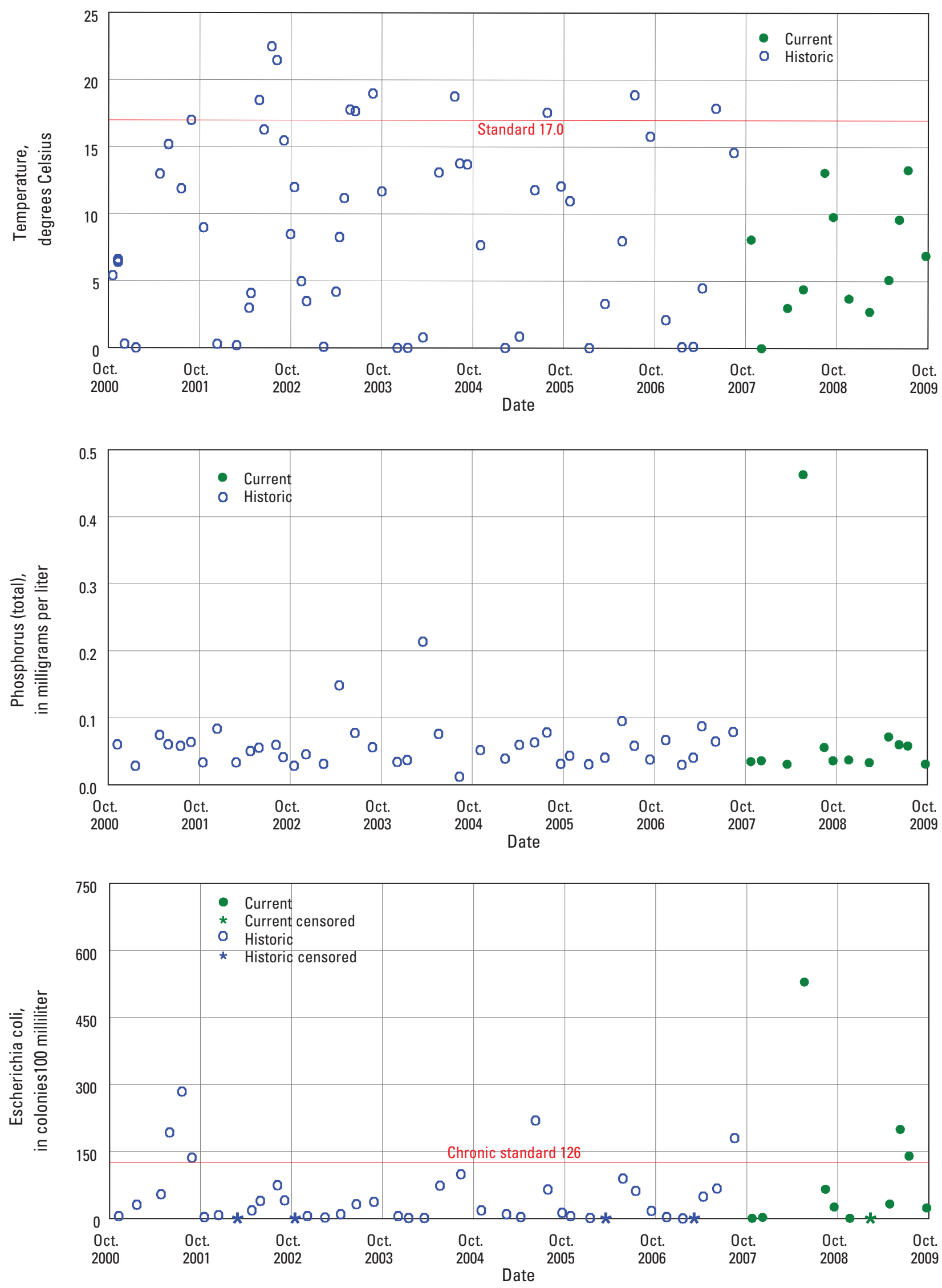

Figure 17. Time-series plots of selected water-quality constituents for Ohio Creek above mouth near Gunnison, Colorado. 
Table 13. Time-series plots of selected water-quality constituents for Ohio Creek above mouth near Gunnison, Colorado, station 09113980.

[--, no data or not applicable; L, low; M, medium; H, high; E. coli, Escherichia coli; $\mathrm{mg} / \mathrm{m}^{2}$, milligrams per square meter; USEPA, U.S. Environmental Protection Agency]

\begin{tabular}{|c|c|c|c|c|c|c|c|c|c|c|c|c|c|c|}
\hline $\begin{array}{c}\text { Property } \\
\text { or } \\
\text { constituent }\end{array}$ & $\begin{array}{l}\text { Period } \\
\text { (water } \\
\text { years) }\end{array}$ & $\begin{array}{l}\text { Number } \\
\text { of } \\
\text { samples }\end{array}$ & $\begin{array}{l}\text { Number } \\
\text { of } \\
\text { censored } \\
\text { values }\end{array}$ & Median' & Maximum & $\begin{array}{c}\text { Date } \\
\text { of } \\
\text { Maximum }\end{array}$ & $\begin{array}{c}\text { 15th } \\
\text { percentile }\end{array}$ & $\begin{array}{c}\text { 85th } \\
\text { percentile }\end{array}$ & $\begin{array}{c}\text { Geometric } \\
\text { Mean }^{2}\end{array}$ & $\begin{array}{c}\text { Standard } \\
\text { or } \\
\text { Chronic } \\
\text { standard }^{3}\end{array}$ & $\begin{array}{l}\text { Number of } \\
\text { exceedences } \\
\text { Standard or } \\
\text { Chronic }\end{array}$ & $\begin{array}{l}\text { Acute } \\
\text { standard }\end{array}$ & $\begin{array}{c}\begin{array}{c}\text { Number } \\
\text { of }\end{array} \\
\text { exceedences } \\
\text { Acute }\end{array}$ & $\begin{array}{c}\text { Level } \\
\text { of } \\
\text { concern }\end{array}$ \\
\hline $\begin{array}{l}\text { Instantaneous discharge, in cubic feet } \\
\text { per second }\end{array}$ & $1997-2007$ & 65 & 0 & 34 & 362 & $05 / 29 / 97$ & 17 & 123 & -- & -- & -- & -- & -- & -- \\
\hline $\begin{array}{l}\text { Instantaneous discharge, in cubic feet } \\
\text { per second }\end{array}$ & 2008-2009 & 13 & 0 & 28 & 807 & $05 / 20 / 08$ & 18 & 221 & -- & -- & -- & -- & -- & -- \\
\hline Dissolved oxygen, in milligrams per liter & 1997-2007 & 62 & 0 & 8.9 & 11.6 & $01 / 19 / 07$ & 7.6 & 10.7 & -- & 6.0 & 0 & -- & -- & $\mathrm{L}$ \\
\hline Dissolved oxygen, in milligrams per liter & 2008-2009 & 12 & 0 & 9.9 & 12.1 & $12 / 04 / 07$ & 8.4 & 10.8 & -- & 6.0 & 0 & -- & -- & $\mathrm{L}$ \\
\hline $\mathrm{pH}$, in standard units & 1997-2007 & 62 & 0 & 8.1 & 8.6 & ${ }^{4} 09 / 04 / 02$ & 7.9 & 8.3 & -- & 6.5 & 0 & 9.0 & -- & $\mathrm{L}$ \\
\hline $\mathrm{pH}$, in standard units & 2008-2009 & 12 & 0 & 8.0 & 8.5 & $10 / 23 / 07$ & 7.5 & 8.4 & -- & 6.5 & 0 & 9.0 & -- & $\mathrm{L}$ \\
\hline $\begin{array}{l}\text { Specific conductance, in microsiemens } \\
\text { per centimeter }\end{array}$ & 1997-2007 & 62 & 0 & 204 & 402 & $10 / 16 / 02$ & 163 & 305 & -- & -- & -- & -- & -- & -- \\
\hline $\begin{array}{l}\text { Specific conductance, in microsiemens } \\
\text { per centimeter }\end{array}$ & 2008-2009 & 12 & 0 & 208 & 362 & $11 / 19 / 08$ & 113 & 317 & -- & -- & -- & -- & -- & -- \\
\hline Temperature, degrees Celsius & $1997-2007$ & 62 & 0 & 8.3 & 21.5 & $08 / 06 / 02$ & 0.2 & 16.9 & -- & 17.0 & 8 & -- & -- & $\mathrm{L}$ \\
\hline Temperature, degrees Celsius & 2008-2009 & 12 & 0 & 6.0 & 13.3 & 07/14/09 & 2.6 & 13.1 & -- & 17.0 & 0 & -- & -- & $\mathrm{L}$ \\
\hline $\begin{array}{l}\text { Ammonia plus organic nitrogen, in mil- } \\
\text { ligrams per liter }\end{array}$ & $1997-2007$ & 62 & 4 & 0.24 & 0.93 & $03 / 17 / 04$ & 0.11 & 0.45 & -- & -- & -- & -- & -- & -- \\
\hline $\begin{array}{l}\text { Ammonia plus organic nitrogen, in mil- } \\
\text { ligrams per liter }\end{array}$ & 2008-2009 & 12 & 0 & 0.18 & 0.89 & $05 / 20 / 08$ & 0.11 & 0.39 & -- & -- & -- & -- & -- & -- \\
\hline $\begin{array}{l}\text { Ammonia nitrogen, in milligrams per } \\
\text { liter }\end{array}$ & 1997-2007 & 62 & 34 & 5 & 0.080 & $03 / 17 / 04$ & 5 & 0.010 & -- & 2.06 & 0 & 4.85 & 0 & $\mathrm{~L}$ \\
\hline $\begin{array}{l}\text { Ammonia nitrogen, in milligrams per } \\
\text { liter }\end{array}$ & 2008-2009 & 12 & 9 & 5 & 0.012 & $04 / 28 / 09$ & 5 & 0.011 & -- & 2.75 & 0 & 7.26 & 0 & $\mathrm{~L}$ \\
\hline $\begin{array}{l}\text { Nitrite plus nitrate nitrogen, in mil- } \\
\text { ligrams per liter }\end{array}$ & 1997-2007 & 62 & 20 & 0.013 & 0.169 & $04 / 16 / 03$ & 5 & 0.064 & -- & 10.0 & 0 & -- & -- & $\mathrm{L}$ \\
\hline $\begin{array}{l}\text { Nitrite plus nitrate nitrogen, in mil- } \\
\text { ligrams per liter }\end{array}$ & 2008-2009 & 12 & 6 & 0.005 & 0.081 & $05 / 20 / 08$ & 5 & 0.073 & -- & 10.0 & 0 & -- & -- & $\mathrm{L}$ \\
\hline Nitrite nitrogen, in milligrams per liter & 1997-2007 & 62 & 34 & 5 & 0.030 & $11 / 22 / 96$ & 5 & 0.002 & -- & 0.05 & 0 & -- & -- & $\mathrm{L}$ \\
\hline Nitrite nitrogen, in milligrams per liter & 2008-2009 & 12 & 9 & 5 & ${ }^{6}<0.002$ & -- & s & 5 & -- & 0.05 & 0 & -- & -- & $\mathrm{L}$ \\
\hline Orthophosphate, in milligrams per liter & $1997-2007$ & 62 & 1 & 0.019 & 0.080 & $03 / 17 / 04$ & 0.012 & 0.028 & -- & -- & -- & -- & -- & -- \\
\hline Orthophosphate, in milligrams per liter & $2008-2009$ & 12 & 0 & 0.021 & 0.030 & $07 / 14 / 09$ & 0.014 & 0.029 & -- & -- & -- & -- & -- & -- \\
\hline Phosphorus (total), in milligrams per liter & $1997-2007$ & 62 & 0 & 0.056 & 0.214 & $03 / 17 / 04$ & 0.031 & 0.083 & -- & -- & -- & -- & -- & -- \\
\hline Phosphorus (total), in milligrams per liter & 2008-2009 & 12 & 0 & 0.037 & 0.463 & $05 / 20 / 08$ & 0.031 & 0.091 & -- & -- & -- & -- & -- & -- \\
\hline E. coli, in colonies per 100 milliliter & $2001-2007$ & 42 & 4 & 18 & 285 & $07 / 19 / 01$ & 2 & 7 & 15.2 & 126 & 5 & -- & -- & $\mathrm{L}$ \\
\hline E. coli, in colonies per 100 milliliter & $2008-2009$ & 11 & 1 & 26 & 530 & $05 / 20 / 08$ & 1 & 7 & -- & 126 & 3 & -- & -- & $\mathrm{L}$ \\
\hline $\begin{array}{l}\text { Biomass periphyton, ashfree drymass, in } \\
\text { grams per square meter }\end{array}$ & 2002-2007 & 6 & 1 & 21.1 & 66.5 & $08 / 06 / 02$ & 0.8 & 64.9 & -- & -- & -- & -- & -- & -- \\
\hline $\begin{array}{l}\text { Biomass periphyton, ashfree drymass, in } \\
\text { grams per square meter }\end{array}$ & 2008-2009 & 2 & 1 & 5.4 & 10.9 & 09/23/09 & 5 & 10.9 & -- & -- & -- & -- & -- & -- \\
\hline
\end{tabular}


Table 13. Time-series plots of selected water-quality constituents for Ohio Creek above mouth near Gunnison, Colorado, station 09113980.—Continued

[--, no data or not applicable; L, low; M, medium; H, high; E. coli, Escherichia coli; mg/m², milligrams per square meter; USEPA, U.S. Environmental Protection Agency]

\begin{tabular}{|c|c|c|c|c|c|c|c|c|c|c|c|c|c|c|}
\hline $\begin{array}{l}\text { Property } \\
\text { or } \\
\text { constituent }\end{array}$ & $\begin{array}{l}\text { Period } \\
\text { (water } \\
\text { years) }\end{array}$ & $\begin{array}{c}\text { Number } \\
\text { of } \\
\text { samples }\end{array}$ & $\begin{array}{l}\text { Number } \\
\text { of } \\
\text { censored } \\
\text { values }\end{array}$ & Median' & Maximum & $\begin{array}{c}\text { Date } \\
\text { of } \\
\text { Maximum }\end{array}$ & $\begin{array}{c}\text { 15th } \\
\text { percentile }\end{array}$ & $\begin{array}{c}\text { 85th } \\
\text { percentile }\end{array}$ & $\begin{array}{l}\text { Geometric } \\
\text { Mean }^{2}\end{array}$ & $\begin{array}{l}\text { Standard } \\
\text { or } \\
\text { Chronic } \\
\text { standard }\end{array}$ & $\begin{array}{l}\text { Number of } \\
\text { exceedences } \\
\text { Standard or } \\
\text { Chronic }\end{array}$ & $\begin{array}{l}\text { Acute } \\
\text { standard }\end{array}$ & $\begin{array}{c}\begin{array}{c}\text { Number } \\
\text { of } \\
\text { exceedences }\end{array} \\
\text { Acute }\end{array}$ & $\begin{array}{l}\text { Level } \\
\text { of } \\
\text { concern }\end{array}$ \\
\hline $\begin{array}{l}\text { Periphyton, biomass, ash weight, in } \\
\text { grams per square meter }\end{array}$ & $2002-2007$ & 6 & 0 & 685 & 1,695 & 08/06/02 & 358 & 1,668 & -- & -- & -- & -- & -- & -- \\
\hline $\begin{array}{l}\text { Periphyton, biomass, ash weight, in } \\
\text { grams per square meter }\end{array}$ & 2008-2009 & 2 & 0 & 264 & 344 & 09/23/09 & 184 & 344 & -- & -- & -- & -- & -- & -- \\
\hline $\begin{array}{l}\text { Periphyton, biomass, dry weight, in } \\
\text { grams per square meter }\end{array}$ & 2002-2007 & 6 & 0 & 706 & 1,762 & 08/06/02 & 365 & 1,733 & -- & -- & -- & -- & -- & -- \\
\hline $\begin{array}{l}\text { Periphyton, biomass, dry weight, in } \\
\text { grams per square meter }\end{array}$ & 2008-2009 & 2 & 0 & 270 & 355 & 09/23/09 & 185 & 355 & -- & -- & -- & -- & -- & -- \\
\hline $\begin{array}{l}\text { Chlorophyll a, periphyton, chromofluoro, } \\
\text { in } \mathrm{mg} / \mathrm{m}^{2}\end{array}$ & 2002-2007 & 6 & 0 & 39.7 & 136 & $08 / 06 / 02$ & 9.30 & 132 & -- & -- & -- & -- & -- & -- \\
\hline $\begin{array}{l}\text { Chlorophyll a, periphyton, chromofluoro, } \\
\text { in } \mathrm{mg} / \mathrm{m}^{2}\end{array}$ & 2008-2009 & 2 & 0 & 23.3 & 43.1 & 09/23/09 & 3.60 & 43.1 & -- & -- & -- & -- & -- & -- \\
\hline Pheophytin a, periphyton, in $\mathrm{mg} / \mathrm{m}^{2}$ & 2002-2007 & 6 & 0 & 15.8 & 53.4 & $08 / 06 / 02$ & 4.1 & 52.0 & -- & -- & -- & -- & -- & -- \\
\hline Pheophytin a, periphyton, in $\mathrm{mg} / \mathrm{m}^{2}$ & 2008-2009 & 2 & 0 & 6.5 & 12.5 & 09/23/09 & 0.5 & 12.5 & -- & -- & -- & -- & -- & -- \\
\hline
\end{tabular}

${ }^{1}$ Censored values were replaced with 0 to compute median and 85 th percentiles (coliform censored values replaced with 1) see definitions section.

${ }^{2}$ Geometric mean listed for E. coli. Geometric mean computed for combined period of record (2001-2009).

${ }^{3}$ Colorado Department of Public Health and Environment, classification and numeric standards for Gunnison and Lower Dolores River Basins (2010a); and USEPA National Recommended Water Quality Criteria (2009). ${ }^{4}$ Multiple dates for maximum.

${ }^{5}$ Median values and percentiles were not tabled when they were calculated as zero.

${ }^{6}$ All values below laboratory reporting level (LRL), current LRL used for current period maximum, highest LRL used for historic period.

${ }^{7}$ Use geometric mean for comparison to standard. 
Table 14. Summary of measured constituents and properties for Ohio Creek above mouth near Gunnison, Colorado, station 09113980.

[E.coli, Escherichia coli]

\begin{tabular}{lcccc}
\hline \multicolumn{1}{c}{ Property or constituent } & $\begin{array}{c}\text { Period } \\
\text { (water years) }\end{array}$ & Kendall tau $(\tau)$ & p-Value & Trend direction \\
\hline Specific conductance, in microsiemens per centimeter & $1999-2009$ & -0.123 & 0.311 & None \\
pH, in standard units & $1999-2009$ & -0.005 & 1.000 & None \\
Ammonia plus organic nitrogen, in milligrams per liter & $1999-2009$ & 0.018 & 0.907 & None \\
Nitrite plus nitrate nitrogen, in milligrams per liter & $1999-2009$ & 0.000 & 1.000 & None \\
Phosphorus (total), in milligrams per liter & $1999-2009$ & -0.150 & 0.213 & None \\
Orthophosphate, in milligrams per liter & $1997-2009$ & -0.043 & 0.707 & None \\
E. coli, in colonies per 100 milliliter & $2001-2009$ & -0.140 & 0.321 & None \\
\hline
\end{tabular}




\section{Gunnison River near Gunnison, Colorado}

Current Reason for Inclusion: This station is upstream from the City of Gunnison wastewater treatment-plant discharge and is part of the rotational group of sites.

General Station Information:

Location:

Station Type:

Period of Record: Water quality:

Streamflow gaging:

Latitude: 383231

Longitude: 1065657
$0.7 \mathrm{mi}$ downstream from Antelope Creek and $1.2 \mathrm{mi}$ west of Gunnison.

The gage is on the right bank.

USGS water quality and streamflow gaging

April 1995-September 2009

October 1944-September 2009

Drainage area: $1,012 \mathrm{mi}^{2}$

HUC: 14020002

\section{USGS Data Summary: Water Years 2008-2009:}

General Chemistry

Nutrients:

E. coli:

Trace Elements/metals:

Other constituents of concern:
$\mathrm{pH}$ : Low concern

Dissolved oxygen: Low concern

Temperature: Low concern

\section{Low concern}

Low concern

No data

None for constituents tested.

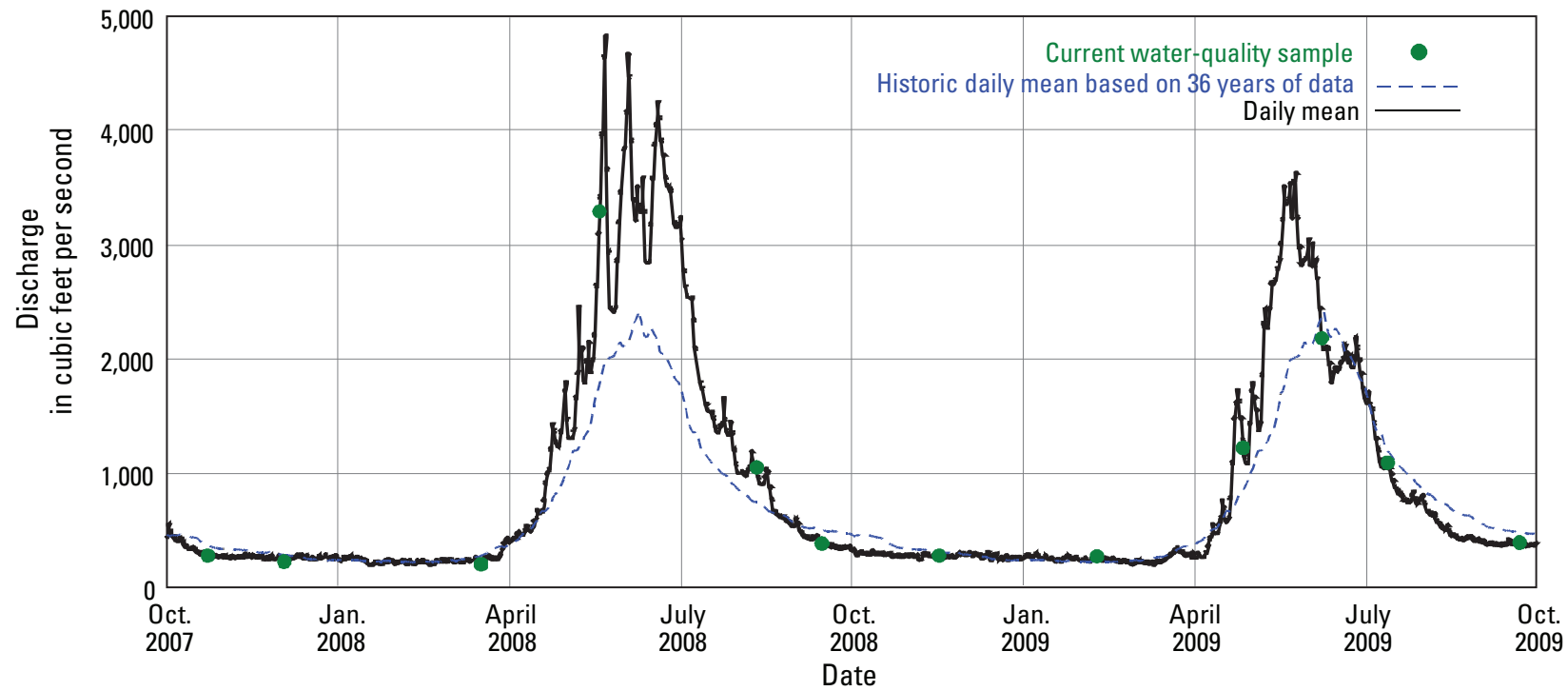

Figure 18. Daily mean discharge and time distribution of water-quality samples for Gunnison River at Gunnison, Colorado. 

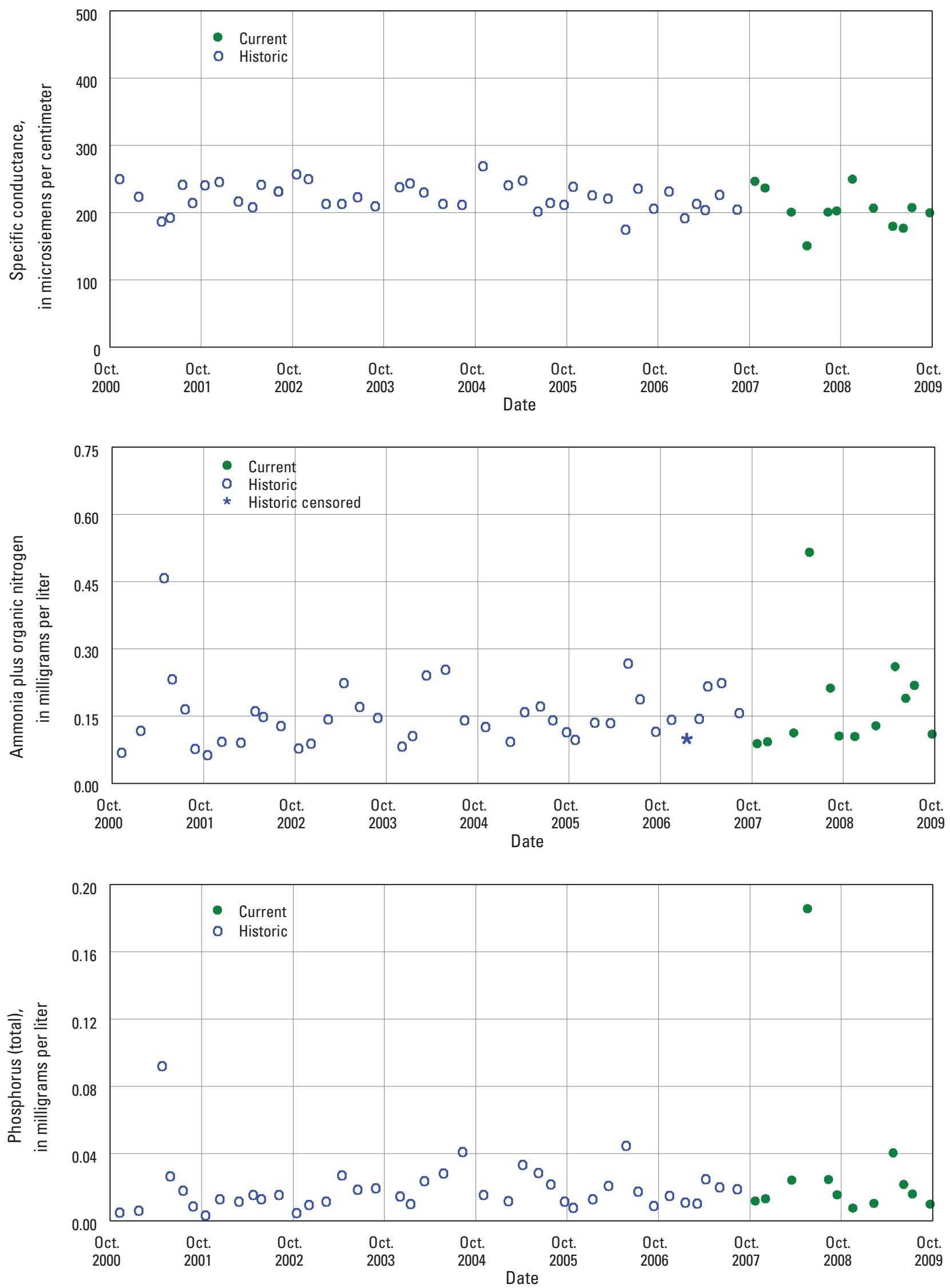

Figure 19. Time-series plots of selected water-quality constituents for Gunnison River at Gunnison, Colorado. 
Table 15. Summary of measured constituents and properties for Gunnison River near Gunnison, Colorado, station 09114500.

[--, no data or not applicable; L, low; M, medium; H, high; E.coli, Escherichia coli; mg $/ \mathrm{m}^{2}$, milligrams per square meter; USEPA, U.S. Environmental Protection Agency]

\begin{tabular}{|c|c|c|c|c|c|c|c|c|c|c|c|c|c|c|}
\hline $\begin{array}{l}\text { Property } \\
\text { or } \\
\text { constituent }\end{array}$ & $\begin{array}{l}\text { Period } \\
\text { (water } \\
\text { years) }\end{array}$ & $\begin{array}{c}\text { Number } \\
\text { of } \\
\text { samples }\end{array}$ & $\begin{array}{c}\text { Number } \\
\text { of } \\
\text { censored } \\
\text { values }\end{array}$ & Median' & Maximum & $\begin{array}{c}\text { Date } \\
\text { of } \\
\text { Maximum }\end{array}$ & $\begin{array}{c}\text { 15th } \\
\text { percentile }\end{array}$ & $\begin{array}{c}\text { 85th } \\
\text { percentile }\end{array}$ & $\begin{array}{c}\text { Geometric } \\
\text { Mean }^{2}\end{array}$ & $\begin{array}{c}\text { Standard } \\
\text { or } \\
\text { Chronic } \\
\text { standard }^{3}\end{array}$ & $\begin{array}{c}\text { Number of } \\
\text { exceedences } \\
\text { Standard or } \\
\text { Chronic }\end{array}$ & $\begin{array}{c}\text { Acute } \\
\text { standard }\end{array}$ & $\begin{array}{c}\begin{array}{c}\text { Number } \\
\text { of } \\
\text { exceedences } \\
\text { Acute }\end{array} \\
\end{array}$ & $\begin{array}{c}\text { Level } \\
\text { of } \\
\text { concern }\end{array}$ \\
\hline $\begin{array}{l}\text { Instantaneous discharge, in } \\
\text { cubic feet per second }\end{array}$ & 1995-2007 & 77 & 0 & 422 & 5,580 & $06 / 19 / 95$ & 210 & 1,601 & -- & -- & -- & -- & -- & -- \\
\hline $\begin{array}{l}\text { Instantaneous discharge, in } \\
\text { cubic feet per second }\end{array}$ & 2008-2009 & 13 & 0 & 383 & 3,290 & $05 / 19 / 08$ & 229 & 2,084 & -- & -- & -- & -- & -- & -- \\
\hline $\begin{array}{l}\text { Dissolved oxygen, in mil- } \\
\text { ligrams per liter }\end{array}$ & 1995-2007 & 75 & 0 & 9.5 & 13.4 & $02 / 10 / 05$ & 8.2 & 11.5 & -- & 6.0 & 0 & -- & -- & $\mathrm{L}$ \\
\hline $\begin{array}{l}\text { Dissolved oxygen, in mil- } \\
\text { ligrams per liter }\end{array}$ & 2008-2009 & 12 & 0 & 9.5 & 12.1 & $12 / 03 / 07$ & 8.4 & 11.8 & -- & 6.0 & 0 & -- & -- & $\mathrm{L}$ \\
\hline $\mathrm{pH}$, in standard units & 1995-2007 & 75 & 0 & 8.3 & 8.8 & ${ }^{4} 03 / 05 / 07$ & 8.1 & 8.5 & -- & 6.5 & 0 & 9.0 & -- & $\mathrm{L}$ \\
\hline $\mathrm{pH}$, in standard units & 2008-2009 & 12 & 0 & 8.1 & 8.8 & $11 / 17 / 08$ & 7.9 & 8.6 & -- & 6.5 & 0 & 9.0 & -- & $\mathrm{L}$ \\
\hline $\begin{array}{l}\text { Specific conductance, in micro- } \\
\text { siemens per centimeter }\end{array}$ & 1995-2007 & 75 & 0 & 213 & 269 & $11 / 02 / 04$ & 184 & 240 & -- & -- & -- & -- & -- & -- \\
\hline $\begin{array}{l}\text { Specific conductance, in micro- } \\
\text { siemens per centimeter }\end{array}$ & 2008-2009 & 12 & 0 & 202 & 250 & $11 / 17 / 08$ & 176 & 247 & -- & -- & -- & -- & -- & -- \\
\hline Temperature, degrees Celsius & 1995-2007 & 75 & 0 & 7.2 & 17.6 & $08 / 13 / 07$ & 0.1 & 13.2 & -- & 17.0 & 1 & -- & -- & $\mathrm{L}$ \\
\hline Temperature, degrees Celsius & $2008-2009$ & 12 & 0 & 7.8 & 16.2 & $08 / 11 / 08$ & 0.2 & 14.5 & -- & 17.0 & 0 & -- & -- & $\mathrm{L}$ \\
\hline $\begin{array}{l}\text { Ammonia plus organic nitro- } \\
\text { gen, in milligrams per liter }\end{array}$ & 1995-2007 & 74 & 20 & 0.13 & 0.46 & $04 / 27 / 01$ & 5 & 0.22 & -- & -- & -- & -- & -- & -- \\
\hline $\begin{array}{l}\text { Ammonia plus organic nitro- } \\
\text { gen, in milligrams per liter }\end{array}$ & 2008-2009 & 12 & 0 & 0.12 & 0.52 & $05 / 19 / 08$ & 0.09 & 0.27 & -- & -- & -- & -- & -- & -- \\
\hline $\begin{array}{l}\text { Ammonia nitrogen, in mil- } \\
\text { ligrams per liter }\end{array}$ & 1995-2007 & 74 & 49 & 5 & 0.051 & $06 / 04 / 98$ & 5 & 0.014 & -- & 1.67 & 0 & 3.66 & 0 & $\mathrm{~L}$ \\
\hline $\begin{array}{l}\text { Ammonia nitrogen, in mil- } \\
\text { ligrams per liter }\end{array}$ & 2008-2009 & 12 & 8 & 5 & 0.015 & $08 / 11 / 08$ & 5 & 0.013 & -- & 2.02 & 0 & 4.73 & 0 & $\mathrm{~L}$ \\
\hline $\begin{array}{l}\text { Nitrite plus nitrate nitrogen, in } \\
\text { milligrams per liter }\end{array}$ & 1995-2007 & 74 & 22 & 0.019 & 0.146 & $06 / 03 / 99$ & 5 & 0.071 & -- & 10.0 & 0 & -- & -- & $\mathrm{L}$ \\
\hline $\begin{array}{l}\text { Nitrite plus nitrate nitrogen, in } \\
\text { milligrams per liter }\end{array}$ & 2008-2009 & 12 & 4 & 0.014 & 0.105 & $05 / 19 / 08$ & 5 & 0.076 & -- & 10.0 & 0 & -- & -- & $\mathrm{L}$ \\
\hline $\begin{array}{l}\text { Nitrite nitrogen, in milligrams } \\
\text { per liter }\end{array}$ & $1995-2007$ & 74 & 55 & 5 & 0.011 & $06 / 04 / 98$ & 5 & 0.001 & -- & 0.05 & 0 & -- & -- & $\mathrm{L}$ \\
\hline $\begin{array}{l}\text { Nitrite nitrogen, in milligrams } \\
\text { per liter }\end{array}$ & 2008-2009 & 12 & 9 & 5 & ${ }^{6}<0.002$ & -- & 5 & 5 & -- & 0.05 & 0 & -- & -- & $\mathrm{L}$ \\
\hline $\begin{array}{l}\text { Orthophosphate, in milligrams } \\
\text { per liter }\end{array}$ & 1995-2007 & 74 & 47 & 5 & 0.030 & $09 / 21 / 95$ & 5 & 0.010 & -- & -- & -- & -- & -- & -- \\
\hline $\begin{array}{l}\text { Orthophosphate, in milligrams } \\
\text { per liter }\end{array}$ & 2008-2009 & 12 & 1 & 0.005 & 0.009 & $04 / 27 / 09$ & 0.003 & 0.008 & -- & -- & -- & -- & -- & -- \\
\hline $\begin{array}{l}\text { Phosphorus (total), in mil- } \\
\quad \text { ligrams per liter }\end{array}$ & 1995-2007 & 73 & 11 & 0.015 & 0.120 & 06/19/95 & 0.000 & 0.030 & -- & -- & -- & -- & -- & -- \\
\hline $\begin{array}{l}\text { Phosphorus (total), in mil- } \\
\text { ligrams per liter }\end{array}$ & 2008-2009 & 12 & 0 & 0.016 & 0.186 & 05/19/08 & 0.010 & 0.048 & -- & -- & -- & -- & -- & -- \\
\hline
\end{tabular}


Table 15. Summary of measured constituents and properties for Gunnison River near Gunnison, Colorado, station 09114500.—Continued

[--, no data or not applicable; L, low; M, medium; H, high; E.coli, Escherichia coli; mg $/ \mathrm{m}^{2}$, milligrams per square meter; USEPA, U.S. Environmental Protection Agency]

\begin{tabular}{|c|c|c|c|c|c|c|c|c|c|c|c|c|c|c|}
\hline $\begin{array}{l}\text { Property } \\
\text { or } \\
\text { constituent }\end{array}$ & $\begin{array}{l}\text { Period } \\
\text { (water } \\
\text { years) }\end{array}$ & $\begin{array}{c}\text { Number } \\
\text { of } \\
\text { samples }\end{array}$ & $\begin{array}{l}\text { Number } \\
\text { of } \\
\text { censored } \\
\text { values }\end{array}$ & Median' & Maximum & $\begin{array}{c}\text { Date } \\
\text { of } \\
\text { Maximum }\end{array}$ & $\begin{array}{c}\text { 15th } \\
\text { percentile }\end{array}$ & $\begin{array}{c}\text { 85th } \\
\text { percentile }\end{array}$ & $\begin{array}{c}\text { Geometric } \\
\text { Mean }^{2}\end{array}$ & $\begin{array}{c}\text { Standard } \\
\text { or } \\
\text { Chronic } \\
\text { standard }\end{array}$ & $\begin{array}{l}\text { Number of } \\
\text { exceedences } \\
\text { Standard or } \\
\text { Chronic }\end{array}$ & $\begin{array}{c}\text { Acute } \\
\text { standard }\end{array}$ & $\begin{array}{c}\begin{array}{c}\text { Number } \\
\text { of }\end{array} \\
\text { exceedences } \\
\text { Acute }\end{array}$ & $\begin{array}{c}\text { Level } \\
\text { of } \\
\text { concern }\end{array}$ \\
\hline $\begin{array}{l}\text { E. coli, in colonies per } 100 \\
\text { milliliter }\end{array}$ & 2001-2007 & 39 & 10 & 7 & 195 & $05 / 25 / 06$ & 1 & 7 & 7.2 & 126 & 2 & -- & -- & $\mathrm{L}$ \\
\hline $\begin{array}{l}\text { E. coli, in colonies per } 100 \\
\text { millililiter }\end{array}$ & 2008-2009 & 11 & 1 & 8 & 78 & 05/19/08 & 1 & 7 & -- & 126 & 0 & -- & -- & $\mathrm{L}$ \\
\hline $\begin{array}{l}\text { Biomass periphyton, ashfree } \\
\text { drymass, in grams per } \\
\text { square meter }\end{array}$ & $2002-2007$ & 6 & 2 & 8.6 & 13.5 & $08 / 07 / 02$ & 5 & 13.5 & -- & -- & -- & -- & -- & -- \\
\hline $\begin{array}{l}\text { Biomass periphyton, ashfree } \\
\text { drymass, in grams per } \\
\text { square meter }\end{array}$ & 2008-2009 & 2 & 1 & 2.8 & 5.6 & 09/22/09 & 5 & 5.6 & -- & -- & -- & -- & -- & -- \\
\hline $\begin{array}{l}\text { Periphyton, biomass, ash } \\
\text { weight, in grams per square } \\
\text { meter }\end{array}$ & $2002-2007$ & 6 & 0 & 431 & 738 & $08 / 28 / 03$ & 291 & 730 & -- & -- & -- & -- & -- & -- \\
\hline $\begin{array}{l}\text { Periphyton, biomass, ash } \\
\text { weight, in grams per square } \\
\text { meter }\end{array}$ & 2008-2009 & 2 & 0 & 240 & 252 & 09/15/08 & 229 & 252 & -- & -- & -- & -- & -- & -- \\
\hline $\begin{array}{l}\text { Periphyton, biomass, dry } \\
\text { weight, in grams per square } \\
\text { meter }\end{array}$ & $2002-2007$ & 6 & 0 & 442 & 749 & $08 / 28 / 03$ & 299 & 741 & -- & -- & -- & -- & -- & -- \\
\hline $\begin{array}{l}\text { Periphyton, biomass, dry } \\
\text { weight, in grams per square } \\
\text { meter }\end{array}$ & 2008-2009 & 2 & 0 & 245 & 255 & 09/15/08 & 235 & 255 & -- & -- & -- & -- & -- & -- \\
\hline $\begin{array}{l}\text { Chlorophyll a, periphyton, } \\
\text { chromofluoro, in } \mathrm{mg} / \mathrm{m}^{2}\end{array}$ & $2002-2007$ & 6 & 0 & 9.10 & 12.6 & $09 / 22 / 05$ & 7.14 & 12.5 & -- & -- & -- & -- & -- & -- \\
\hline $\begin{array}{l}\text { Chlorophyll a, periphyton, } \\
\text { chromofluoro, in } \mathrm{mg} / \mathrm{m}^{2}\end{array}$ & 2008-2009 & 2 & 0 & 8.69 & 9.31 & 09/15/08 & 8.07 & 9.31 & -- & -- & -- & -- & -- & -- \\
\hline $\begin{array}{l}\text { Pheophytin a, periphyton, in } \\
\mathrm{mg} / \mathrm{m}^{2}\end{array}$ & 2002-2007 & 6 & 0 & 4.6 & 5.8 & 08/10/04 & 0.3 & 5.8 & -- & -- & -- & -- & -- & -- \\
\hline $\begin{array}{l}\text { Pheophytin a, periphyton, in } \\
\mathrm{mg} / \mathrm{m}^{2}\end{array}$ & 2008-2009 & 2 & 0 & 2.8 & 2.9 & 09/22/09 & 2.8 & 2.9 & -- & -- & -- & -- & -- & -- \\
\hline
\end{tabular}

${ }^{2}$ Geometric mean listed for $E$. coli. Geometric mean computed for combined period of record (2001-2009).

${ }^{3}$ Colorado Department of Public Health and Environment, classification and numeric standards for Gunnison and Lower Dolores River Basins (2010a); and USEPA National Recommended Water Quality Criteria (2009). ${ }^{4}$ Multiple dates for maximum.

${ }^{5}$ Median values and percentiles were not tabled when they were calculated as zero.

${ }^{6}$ All values below laboratory reporting level (LRL), current LRL used for current period maximum, highest LRL used for historic period.

${ }^{7}$ Use geometric mean for comparison to standard. 
Table 16. Summary of seasonal Kendall trend tests for Gunnison River near Gunnison, Colorado, station 09114500.

[E. coli, Escherichia coli]

\begin{tabular}{lcccc}
\hline \multicolumn{1}{c}{ Property or constituent } & $\begin{array}{c}\text { Period } \\
\text { (water years) }\end{array}$ & Kendall tau $(\tau)$ & p-Value & Trend direction \\
\hline Specific conductance, in microsiemens per centimeter & $1995-2009$ & 0.208 & 0.038 & Up \\
pH, in standard units & $1995-2009$ & 0.121 & 0.230 & None \\
Ammonia plus organic nitrogen, in milligrams per liter & $1999-2009$ & 0.273 & 0.021 & Up \\
Nitrite plus nitrate nitrogen, in milligrams per liter & $2000-2009$ & -0.089 & 0.492 & None \\
Phosphorus (total), in milligrams per liter & $2000-2009$ & 0.133 & 0.303 & None \\
E. coli, in colonies per 100 milliliter & $2001-2009$ & -0.081 & 0.569 & None \\
\hline
\end{tabular}




\section{Tomichi Creek below Cochetopa Creek, Colorado}

Current Reason for Inclusion: This station is downstream from Cochetopa Creek and is part of the rotational group of sites.

\section{General Station Information:}

Location:

Station Type:

Period of Record:

Latitude: 383126

Longitude: 1064756
1 mi downstream from confluence with Cochetopa Creek and 4 mi northwest of Parlin.

USGS water quality

Water quality: November 2000-September 2009

Drainage area: $984 \mathrm{mi}^{2}$

HUC: 14020003

USGS Data Summary: Water Years 2008-2009:

General chemistry

pH: Low concern

Dissolved oxygen: Low concern

Temperature:

Low concern

Nutrients:

Low concern

E. coli:

Low concern

Trace Elements/metals:

Low concern

Other constituents of concern: $\quad$ None for constituents tested.

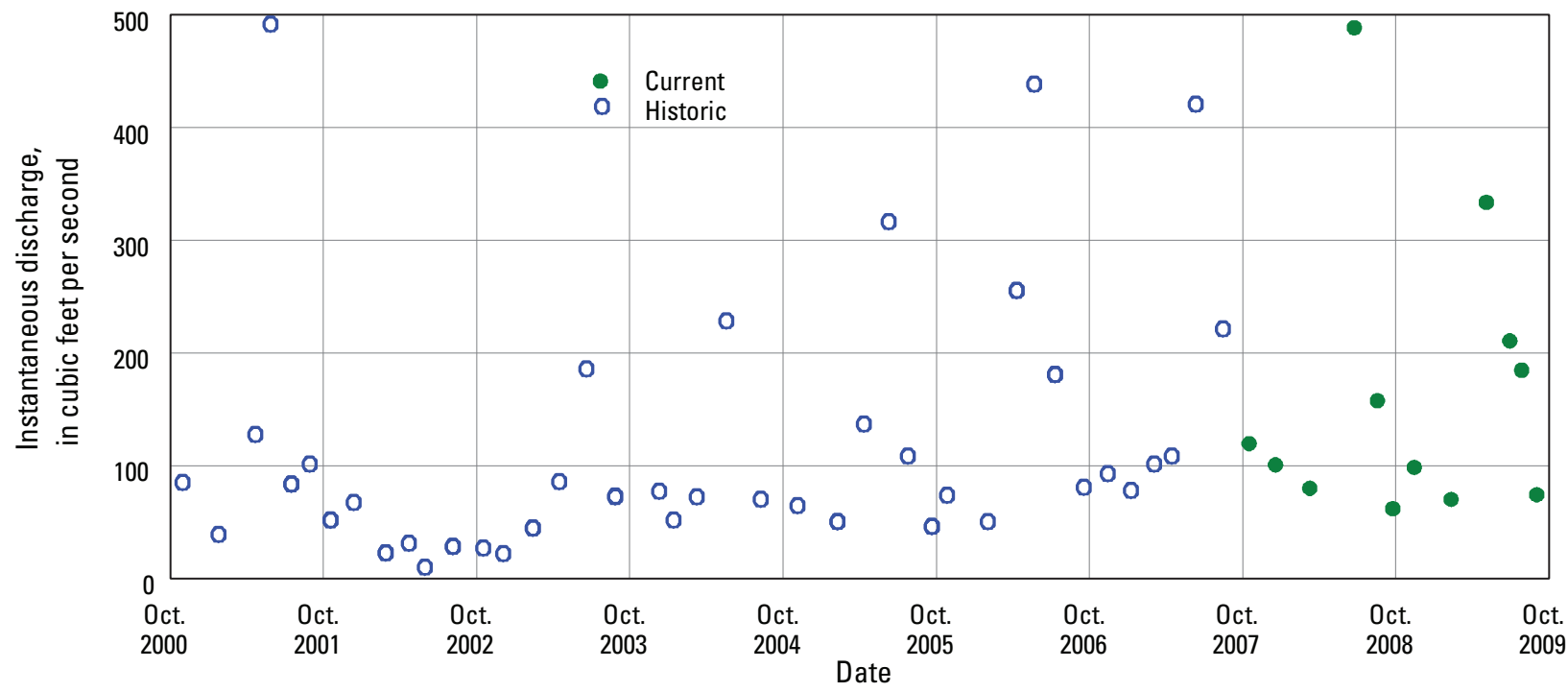

Figure 20. Time distribution and associated discharge of water-quality samples for Tomichi Creek below Cochetopa Creek, Colorado. 

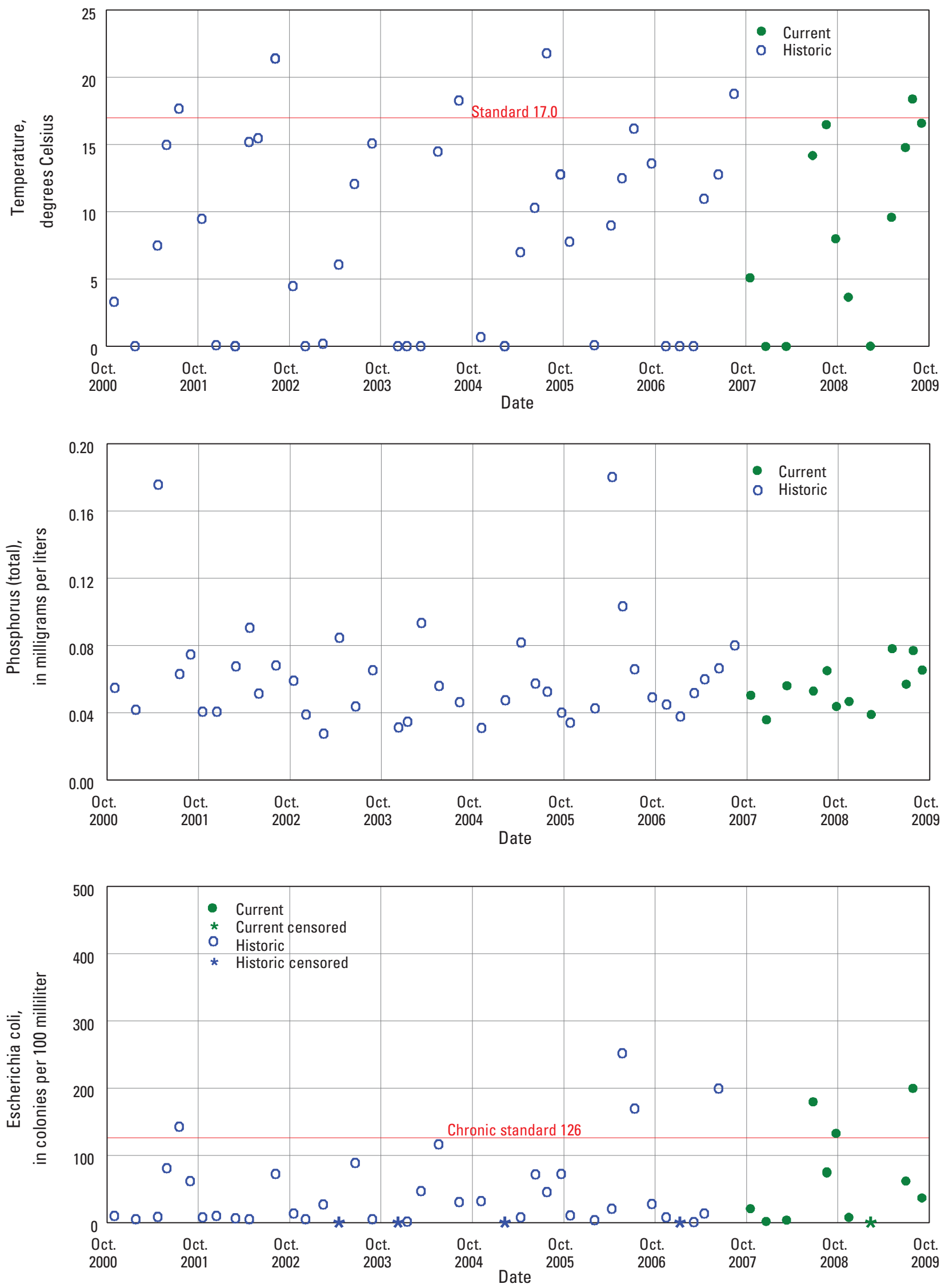

Figure 21. Time-series plots of selected water-quality constituents for Tomichi Creek below Cochetopa Creek, Colorado. 
Table 17. Summary of measured constituents and properties for Tomichi Creek below Cochetopa Creek, Colorado.

[--, no data or not applicable; L, low; M, medium; H, high; NTRU, Nephelometric Turbidity Ratio Unit; E. coli, Escherichia coli; mg/m² milligrams per square meter; USEPA, U.S. Environmental Protection Agency]

\begin{tabular}{|c|c|c|c|c|c|c|c|c|c|c|c|c|c|c|}
\hline $\begin{array}{l}\text { Property } \\
\text { or } \\
\text { constituent }\end{array}$ & $\begin{array}{l}\text { Period } \\
\text { (water } \\
\text { years) }\end{array}$ & $\begin{array}{c}\text { Number } \\
\text { of } \\
\text { samples }\end{array}$ & $\begin{array}{l}\text { Number } \\
\text { of } \\
\text { censored } \\
\text { values }\end{array}$ & Median ${ }^{1}$ & Maximum & $\begin{array}{c}\text { Date } \\
\text { of } \\
\text { Maximum }\end{array}$ & $\begin{array}{c}\text { 15th } \\
\text { percentile }\end{array}$ & $\begin{array}{c}\text { 85th } \\
\text { percentile }\end{array}$ & $\begin{array}{c}\text { Geometric } \\
\text { Mean }^{2}\end{array}$ & $\begin{array}{c}\text { Standard } \\
\text { or } \\
\text { Chronic } \\
\text { standard }\end{array}$ & $\begin{array}{l}\text { Number of } \\
\text { exceedences } \\
\text { Standard or } \\
\text { Chronic }\end{array}$ & $\begin{array}{l}\text { Acute } \\
\text { standard }\end{array}$ & $\begin{array}{c}\begin{array}{c}\text { Number } \\
\text { of } \\
\text { exceedences } \\
\text { Acute }\end{array} \\
\text { a }\end{array}$ & $\begin{array}{l}\text { Level } \\
\text { of } \\
\text { concern }\end{array}$ \\
\hline $\begin{array}{l}\text { Instantaneous discharge, in cubic feet } \\
\text { per second }\end{array}$ & $1998-2007$ & 53 & 0 & 77 & 492 & $05 / 29 / 01$ & 32 & 218 & -- & -- & -- & -- & -- & -- \\
\hline $\begin{array}{l}\text { Instantaneous discharge, in cubic feet } \\
\text { per second }\end{array}$ & 2008-2009 & 12 & 0 & 111 & 489 & $06 / 24 / 08$ & 70 & 342 & -- & -- & -- & -- & -- & -- \\
\hline Turbidity, in NTRU & 2001-2007 & 27 & 1 & 3.8 & 37.1 & $04 / 23 / 01$ & 2.1 & 9.4 & -- & -- & -- & -- & -- & -- \\
\hline Turbidity, in NTRU & 2008-2009 & 8 & 0 & 3.0 & 15.1 & 05/05/09 & 2.1 & 11.4 & -- & -- & -- & -- & -- & -- \\
\hline $\begin{array}{l}\text { Dissolved oxygen, in milligrams per } \\
\text { liter }\end{array}$ & 1998-2007 & 44 & 0 & 9.4 & 11.9 & 01/09/07 & 8.2 & 10.6 & -- & 6.0 & 0 & -- & -- & $\mathrm{L}$ \\
\hline $\begin{array}{l}\text { Dissolved oxygen, in milligrams per } \\
\text { liter }\end{array}$ & 2008-2009 & 12 & 0 & 8.4 & 10.1 & $11 / 14 / 08$ & 7.8 & 9.6 & -- & 6.0 & 0 & -- & -- & $\mathrm{L}$ \\
\hline $\mathrm{pH}$, in standard units & 1998-2007 & 45 & 0 & 8.2 & 9.0 & $07 / 27 / 05$ & 7.8 & 8.5 & -- & 6.5 & 0 & 9.0 & -- & $\mathrm{L}$ \\
\hline $\mathrm{pH}$, in standard units & 2008-2009 & 12 & 0 & 7.9 & 8.5 & 08/18/08 & 7.5 & 8.5 & -- & 6.5 & 0 & 9.0 & -- & $\mathrm{L}$ \\
\hline $\begin{array}{l}\text { Specific conductance, in microsiemens } \\
\text { per centimeter }\end{array}$ & 1998-2007 & 45 & 0 & 260 & 420 & $05 / 31 / 02$ & 230 & 319 & -- & -- & -- & -- & -- & -- \\
\hline $\begin{array}{l}\text { Specific conductance, in microsiemens } \\
\text { per centimeter }\end{array}$ & 2008-2009 & 12 & 0 & 240 & 308 & 06/30/09 & 219 & 288 & -- & -- & -- & -- & -- & -- \\
\hline Temperature, degrees Celsius & $1998-2007$ & 46 & 0 & 8.4 & 21.8 & $07 / 27 / 05$ & 0.0 & 16.2 & -- & 17.0 & 6 & -- & -- & $\mathrm{L}$ \\
\hline Temperature, degrees Celsius & 2008-2009 & 12 & 0 & 8.8 & 18.4 & 07/28/09 & 0.0 & 16.7 & -- & 17.0 & 1 & -- & -- & $\mathrm{L}$ \\
\hline Calcium, in milligrams per liter & $1998-2007$ & 29 & 0 & 35.2 & 53.8 & $05 / 31 / 02$ & 27.8 & 44.7 & -- & -- & -- & -- & -- & -- \\
\hline Calcium, in milligrams per liter & 2008-2009 & 8 & 0 & 31.5 & 42.9 & 06/30/09 & 25.2 & 41.8 & -- & -- & -- & -- & -- & -- \\
\hline Magnesium, in milligrams per liter & 1998-2007 & 29 & 0 & 9.00 & 14.9 & $05 / 31 / 02$ & 6.95 & 11.7 & -- & -- & -- & -- & -- & -- \\
\hline Magnesium, in milligrams per liter & 2008-2009 & 8 & 0 & 8.18 & 11.8 & 06/30/09 & 6.89 & 11.4 & -- & -- & -- & -- & -- & -- \\
\hline $\begin{array}{l}\text { Ammonia plus organic nitrogen, in } \\
\text { milligrams per liter }\end{array}$ & 1998-2007 & 42 & 0 & 0.25 & 0.79 & $05 / 24 / 06$ & 0.14 & 0.54 & -- & -- & -- & -- & -- & -- \\
\hline $\begin{array}{l}\text { Ammonia plus organic nitrogen, in } \\
\text { milligrams per liter }\end{array}$ & 2008-2009 & 12 & 0 & 0.22 & 0.46 & 06/30/09 & 0.17 & 0.44 & -- & -- & -- & -- & -- & -- \\
\hline $\begin{array}{l}\text { Ammonia nitrogen, in milligrams } \\
\text { per liter }\end{array}$ & $1998-2007$ & 43 & 28 & 4 & 0.051 & $03 / 17 / 98$ & 4 & 0.009 & -- & 1.92 & 0 & 4.51 & 0 & $\mathrm{~L}$ \\
\hline $\begin{array}{l}\text { Ammonia nitrogen, in milligrams } \\
\text { per liter }\end{array}$ & 2008-2009 & 12 & 10 & 4 & 0.015 & 02/10/09 & 4 & 0.013 & -- & 2.62 & 0 & 7.19 & 0 & $\mathrm{~L}$ \\
\hline $\begin{array}{l}\text { Nitrite plus nitrate nitrogen, in mil- } \\
\text { ligrams per liter }\end{array}$ & $1998-2007$ & 43 & 27 & ${ }^{4}$ & 0.105 & $02 / 02 / 06$ & 4 & 0.067 & -- & 10.0 & 0 & -- & -- & $\mathrm{L}$ \\
\hline $\begin{array}{l}\text { Nitrite plus nitrate nitrogen, in mil- } \\
\text { ligrams per liter }\end{array}$ & 2008-2009 & 12 & 8 & 4 & 0.085 & 02/10/09 & 4 & 0.049 & -- & 10.0 & 0 & -- & -- & $\mathrm{L}$ \\
\hline Nitrite nitrogen, in milligrams per liter & $1998-2007$ & 43 & 29 & 4 & 0.003 & $02 / 09 / 05$ & 4 & 0.001 & -- & 0.05 & 0 & -- & -- & $\mathrm{L}$ \\
\hline Nitrite nitrogen, in milligrams per liter & 2008-2009 & 12 & 9 & 4 & 0.001 & $02 / 10 / 09$ & 4 & 0.001 & -- & 0.05 & 0 & -- & -- & $\mathrm{L}$ \\
\hline
\end{tabular}


[--, no data or not applicable; L, low; M, medium; H, high; NTRU, Nephelometric Turbidity Ratio Unit; E. coli, Escherichia coli; mg/m², milligrams per square meter; USEPA, U.S. Environmental Protection Agency]

\begin{tabular}{|c|c|c|c|c|c|c|c|c|c|c|c|c|c|c|}
\hline $\begin{array}{c}\text { Property } \\
\text { or } \\
\text { constituent }\end{array}$ & $\begin{array}{l}\text { Period } \\
\text { (water } \\
\text { years) }\end{array}$ & $\begin{array}{l}\text { Number } \\
\text { of } \\
\text { samples }\end{array}$ & $\begin{array}{c}\text { Number } \\
\text { of } \\
\text { censored } \\
\text { values }\end{array}$ & Median' & Maximum & $\begin{array}{c}\text { Date } \\
\text { of } \\
\text { Maximum }\end{array}$ & $\begin{array}{c}\text { 15th } \\
\text { percentile }\end{array}$ & $\begin{array}{c}\text { 85th } \\
\text { percentile }\end{array}$ & $\begin{array}{c}\text { Geometric } \\
\text { Mean }^{2}\end{array}$ & $\begin{array}{c}\text { Standard } \\
\text { or } \\
\text { Chronic } \\
\text { standard }^{3}\end{array}$ & $\begin{array}{l}\text { Number of } \\
\text { exceedences } \\
\text { Standard or } \\
\text { Chronic }\end{array}$ & $\begin{array}{l}\text { Acute } \\
\text { standard }\end{array}$ & $\begin{array}{c}\begin{array}{c}\text { Number } \\
\text { of } \\
\text { exceedences }\end{array} \\
\text { Acute }\end{array}$ & $\begin{array}{l}\text { Level } \\
\text { of } \\
\text { concern }\end{array}$ \\
\hline Orthophosphate, in milligrams per liter & 1998-2007 & 43 & 0 & 0.023 & 0.055 & $04 / 24 / 02$ & 0.011 & 0.036 & -- & -- & -- & -- & -- & -- \\
\hline Orthophosphate, in milligrams per liter & $2008-2009$ & 12 & 0 & 0.024 & 0.040 & 09/02/09 & 0.017 & 0.036 & -- & -- & -- & -- & -- & -- \\
\hline $\begin{array}{l}\text { Phosphorus (total), in milligrams } \\
\text { per liter }\end{array}$ & 1998-2007 & 42 & 0 & 0.056 & 0.180 & 04/11/06 & 0.038 & 0.088 & -- & -- & -- & -- & -- & -- \\
\hline $\begin{array}{l}\text { Phosphorus (total), in milligrams } \\
\text { per liter }\end{array}$ & 2008-2009 & 12 & 0 & 0.055 & 0.078 & 05/05/09 & 0.039 & 0.077 & -- & -- & -- & -- & -- & -- \\
\hline E. coli, in colonies per 100 milliliter & $2001-2007$ & 39 & 4 & 14 & 252 & $05 / 24 / 06$ & 2.00 & 5 & 16.7 & 126 & 4 & -- & -- & $\mathrm{L}$ \\
\hline E. coli, in colonies per 100 milliliter & 2008-2009 & 11 & 1 & 37 & 200 & 07/28/09 & 1.80 & 5 & -- & 126 & 3 & -- & -- & $\mathrm{L}$ \\
\hline $\begin{array}{l}\text { Biomass periphyton, ashfree drymass, } \\
\text { in grams per square meter }\end{array}$ & $1998-2007$ & 14 & 1 & 19.4 & 73.2 & 02/09/05 & 7.0 & 27.9 & -- & -- & -- & -- & -- & -- \\
\hline $\begin{array}{l}\text { Biomass periphyton, ashfree drymass, } \\
\text { in grams per square meter }\end{array}$ & $2008-2009$ & 3 & 1 & 15.5 & 35.7 & 09/02/09 & 4 & 35.7 & -- & -- & -- & -- & -- & -- \\
\hline $\begin{array}{l}\text { Periphyton, biomass, ash weight, in } \\
\text { grams per square meter }\end{array}$ & $1998-2007$ & 14 & 0 & 632 & 842 & $09 / 21 / 05$ & 206 & 836 & -- & -- & -- & -- & -- & -- \\
\hline $\begin{array}{l}\text { Periphyton, biomass, ash weight, in } \\
\text { grams per square meter }\end{array}$ & $2008-2009$ & 3 & 0 & 376 & 1,020 & 09/02/09 & 229 & 1,020 & -- & -- & -- & -- & -- & -- \\
\hline $\begin{array}{l}\text { Periphyton, biomass, dry weight, in } \\
\text { grams per square meter }\end{array}$ & $1998-2007$ & 14 & 0 & 659 & 910 & 02/09/05 & 220 & 860 & -- & -- & -- & -- & -- & -- \\
\hline $\begin{array}{l}\text { Periphyton, biomass, dry weight, in } \\
\text { grams per square meter }\end{array}$ & $2008-2009$ & 3 & 0 & 391 & 1,056 & 09/02/09 & 234 & 1,056 & -- & -- & -- & -- & -- & -- \\
\hline $\begin{array}{l}\text { Chlorophyll a, periphyton, chromo- } \\
\text { fluoro, in } \mathrm{mg} / \mathrm{m}^{2}\end{array}$ & 1998-2007 & 15 & 0 & 27.5 & 133 & 02/09/05 & 9.83 & 78.7 & -- & -- & -- & -- & -- & -- \\
\hline $\begin{array}{l}\text { Chlorophyll a, periphyton, chromo- } \\
\text { fluoro, in } \mathrm{mg} / \mathrm{m}^{2}\end{array}$ & 2008-2009 & 3 & 0 & 36.3 & 45.3 & 09/02/09 & 31.0 & 45.3 & -- & -- & -- & -- & -- & -- \\
\hline Pheophytin a, periphyton, in $\mathrm{mg} / \mathrm{m}^{2}$ & 2001-2007 & 13 & 0 & 16.1 & 39.5 & $01 / 15 / 04$ & 2.3 & 25.8 & -- & -- & -- & -- & -- & -- \\
\hline Pheophytin a, periphyton, in $\mathrm{mg} / \mathrm{m}^{2}$ & 2008-2009 & 3 & 0 & 11.0 & 26.6 & 09/02/09 & 6.6 & 26.6 & -- & -- & -- & -- & -- & -- \\
\hline Aluminum, in micrograms per liter & 2001-2007 & 27 & 11 & 1.1 & 4.8 & $04 / 16 / 07$ & 4 & 2.5 & -- & -- & -- & -- & -- & -- \\
\hline Aluminum, in micrograms per liter & 2008-2009 & 8 & 1 & 3.9 & 16.1 & 05/05/09 & 1.0 & 12.4 & -- & -- & -- & -- & -- & -- \\
\hline Cadmium, in micrograms per liter & 2001-2007 & 27 & 23 & 4 & ${ }^{6}<0.2$ & -- & 4 & 4 & -- & 0.46 & 0 & 1.89 & 0 & $\mathrm{~L}$ \\
\hline Cadmium, in micrograms per liter & 2008-2009 & 8 & 3 & 4 & ${ }^{6}<0.04$ & -- & 4 & 4 & -- & 0.46 & 0 & 1.89 & 0 & $\mathrm{~L}$ \\
\hline Copper, in micrograms per liter & 2001-2007 & 27 & 6 & 0.82 & 4.7 & $05 / 24 / 06$ & 4 & 1.8 & -- & 9.9 & 0 & 15.0 & 0 & $\mathrm{~L}$ \\
\hline Copper, in micrograms per liter & 2008-2009 & 8 & 0 & 1.1 & 3.9 & $10 / 17 / 07$ & 0.61 & 3.1 & -- & 9.9 & 0 & 15.0 & 0 & $\mathrm{~L}$ \\
\hline Iron, in micrograms per liter & 1998-2007 & 29 & 0 & 73 & 157 & $04 / 17 / 03$ & 45 & 98 & -- & 300 & 0 & -- & -- & $\mathrm{L}$ \\
\hline Iron, in micrograms per liter & 2008-2009 & 8 & 0 & 93 & 155 & 06/30/09 & 56 & 149 & -- & 300 & 0 & -- & -- & $\mathrm{L}$ \\
\hline Lead, in micrograms per liter & 2001-2007 & 27 & 12 & 0.05 & 0.66 & $06 / 20 / 03$ & 4 & 0.16 & -- & 2.86 & 0 & 73.5 & 0 & $\mathrm{~L}$ \\
\hline Lead, in micrograms per liter & 2008-2009 & 8 & 1 & 0.18 & 0.30 & 05/05/09 & 0.03 & 0.29 & -- & 2.86 & 0 & 73.5 & 0 & $\mathrm{~L}$ \\
\hline
\end{tabular}


Table 17. Summary of measured constituents and properties for Tomichi Creek below Cochetopa Creek, Colorado.

[--, no data or not applicable; L, low; M, medium; H, high; NTRU, Nephelometric Turbidity Ratio Unit; E. coli, Escherichia coli; mg/m², milligrams per square meter; USEPA, U.S. Environmental Protection Agency]

\begin{tabular}{|c|c|c|c|c|c|c|c|c|c|c|c|c|c|c|}
\hline $\begin{array}{l}\text { Property } \\
\text { or } \\
\text { constituent }\end{array}$ & $\begin{array}{l}\text { Period } \\
\text { (water } \\
\text { years) }\end{array}$ & $\begin{array}{c}\text { Number } \\
\text { of } \\
\text { samples }\end{array}$ & $\begin{array}{c}\text { Number } \\
\text { of } \\
\text { censored } \\
\text { values }\end{array}$ & Median ${ }^{1}$ & Maximum & $\begin{array}{c}\text { Date } \\
\text { of } \\
\text { Maximum }\end{array}$ & $\begin{array}{c}\text { 15th } \\
\text { percentile }\end{array}$ & $\begin{array}{c}\text { 85th } \\
\text { percentile }^{1}\end{array}$ & $\begin{array}{c}\text { Geometric } \\
\text { Mean }^{2}\end{array}$ & $\begin{array}{c}\text { Standard } \\
\text { or } \\
\text { Chronic } \\
\text { standard }^{3}\end{array}$ & $\begin{array}{l}\text { Number of } \\
\text { exceedences } \\
\text { Standard or } \\
\text { Chronic }\end{array}$ & $\begin{array}{c}\text { Acute } \\
\text { standard }\end{array}$ & $\begin{array}{c}\begin{array}{c}\text { Number } \\
\text { of } \\
\text { exceedences } \\
\text { Acute }\end{array} \\
\text { a }\end{array}$ & $\begin{array}{c}\text { Level } \\
\text { of } \\
\text { concern }\end{array}$ \\
\hline Manganese, in micrograms per liter & 1998-2007 & 29 & 0 & 36.3 & 117 & $05 / 31 / 02$ & 15.0 & 62.7 & -- & 1,716 & 0 & 3,106 & 0 & $\mathrm{~L}$ \\
\hline Manganese, in micrograms per liter & 2008-2009 & 8 & 0 & 34.3 & 63.9 & 06/30/09 & 20.5 & 58.7 & -- & 1,716 & 0 & 3,106 & 0 & $\mathrm{~L}$ \\
\hline Silver, in micrograms per liter & $2001-2007$ & 27 & 27 & 4 & ${ }^{6}<0.3$ & -- & 4 & 4 & -- & 0.09 & 0 & 2.49 & 0 & $\mathrm{~L}$ \\
\hline Silver, in micrograms per liter & $2008-2009$ & 8 & 8 & 4 & ${ }^{6}<0.1$ & -- & 4 & 4 & -- & 0.09 & 0 & 2.49 & 0 & $\mathrm{~L}$ \\
\hline Zinc, in micrograms per liter & $2001-2007$ & 27 & 9 & 0.67 & 13.2 & 04/23/01 & 4 & 3.8 & -- & 138 & 0 & 159 & 0 & $\mathrm{~L}$ \\
\hline Zinc, in micrograms per liter & $2008-2009$ & 8 & 1 & 2.2 & 4.0 & $02 / 10 / 09$ & 0.44 & 3.7 & -- & 138 & 0 & 159 & 0 & $\mathrm{~L}$ \\
\hline $\begin{array}{l}\text { Suspended sediment, in milligrams } \\
\text { per liter }\end{array}$ & 1998-2007 & 29 & 0 & 6.0 & 104 & $04 / 23 / 01$ & 3.0 & 21.1 & -- & -- & -- & -- & -- & -- \\
\hline $\begin{array}{l}\text { Suspended sediment, in milligrams } \\
\text { per liter }\end{array}$ & 2008-2009 & 8 & 0 & 5.5 & 31.0 & 05/05/09 & 3.4 & 24.4 & -- & -- & -- & -- & -- & -- \\
\hline
\end{tabular}

${ }^{1}$ Censored values were replaced with 0 to compute median and 85 th percentiles (coliform censored values replaced with 1 ) see definitions section.

${ }^{2}$ Geometric mean listed for E. coli. Geometric mean computed for combined period of record (2001-2009).

${ }^{3}$ Colorado Department of Public Health and Environment, classification and numeric standards for Gunnison and Lower Dolores River Basins (2010a); and USEPA National Recommended Water Quality Criteria (2009).

${ }^{4}$ Median values and percentiles were not tabled when they were calculated as zero.

${ }^{5}$ All values below laboratory reporting level (LRL), current LRL used for current period maximum, highest LRL used for historic period.

${ }^{6}$ Use geometric mean for comparison to standard. 
Table 18. Summary of seasonal Kendall trend tests for Tomichi Creek below Cochetopa Creek, Colorado, station 383126106475600.

[E. coli, Escherichia coli]

\begin{tabular}{|c|c|c|c|c|}
\hline Property or constituent & $\begin{array}{c}\text { Period } \\
\text { (water years) }\end{array}$ & Kendall tau $(\tau)$ & p-Value & Trend direction \\
\hline Specific conductance, in microsiemens per centimeter & $2001-2009$ & -0.285 & 0.036 & Down \\
\hline $\mathrm{pH}$, in standard units & $2001-2009$ & -0.222 & 0.104 & None \\
\hline Ammonia plus organic nitrogen, in milligrams per liter & $2001-2009$ & 0.069 & 0.638 & None \\
\hline Phosphorus (total), in milligrams per liter & $2001-2009$ & -0.139 & 0.322 & None \\
\hline Orthophosphate, in milligrams per liter & $2001-2009$ & -0.069 & 0.639 & None \\
\hline Calcium, in milligrams per liter & $2001-2009$ & -0.156 & 0.376 & None \\
\hline Magnesium, in milligrams per liter & $2001-2009$ & -0.067 & 0.734 & None \\
\hline Copper, in micrograms per liter & 2001-2009 & 0.100 & 0.583 & None \\
\hline Iron, in micrograms per liter & 2001-2009 & 0.222 & 0.196 & None \\
\hline Manganese, in micrograms per liter & 2001-2009 & -0.022 & 0.946 & None \\
\hline Zinc, in micrograms per liter & 2003-2009 & 0.078 & 0.754 & None \\
\hline Suspended sediment, in milligrams per liter & 2001-2009 & 0.056 & 0.784 & None \\
\hline E. coli, in colonies per 100 milliliter & 2001-2009 & 0.074 & 0.623 & None \\
\hline
\end{tabular}




\section{Tomichi Creek at Gunnison, Colorado}

Current Reason for Inclusion: This station represents contribution of the entire Tomichi Creek Basin. Improves the spatial distribution of the network. Long-term monitoring.

General Station Information:

Location:

Station Type:

Period of Record:

Streamflow gaging:

Latitude: 383118

Longitude: 1065625
$300 \mathrm{ft}$ downstream from highway bridge, 1.8 mi southwest of U.S. Post Office in Gunnison and 2.0 mi upstream from mouth.

USGS water quality and streamflow gaging site

Water quality: October 1990-September 1993

April 1995-September 2009

October 1937-September 2009

Drainage area: $1,061 \mathrm{mi}^{2}$

Stream segment: 18
HUC: 14020003

USGS Data Summary: Water Years 2008-2009:

General Chemistry:

$\mathrm{pH}$ : Low concern

Dissolved oxygen: Low concern

Temperature: High concern

Nutrients:

Low concern

E. coli:

Low concern

Trace Elements/metals:

Low concern

Other constituents of concern: None for constituents tested.

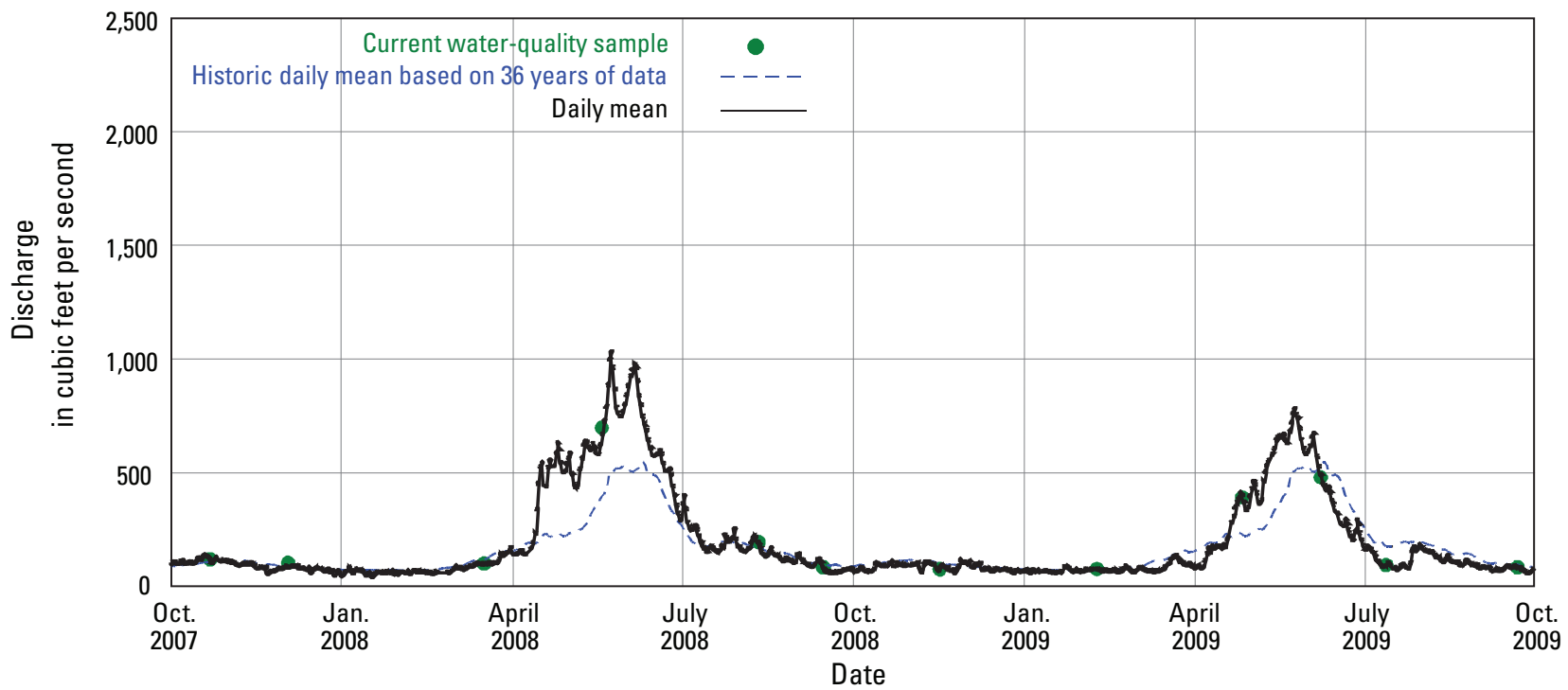

Figure 22. Daily mean discharge and time distribution of water-quality samples for Tomichi Creek at Gunnison, Colorado. 

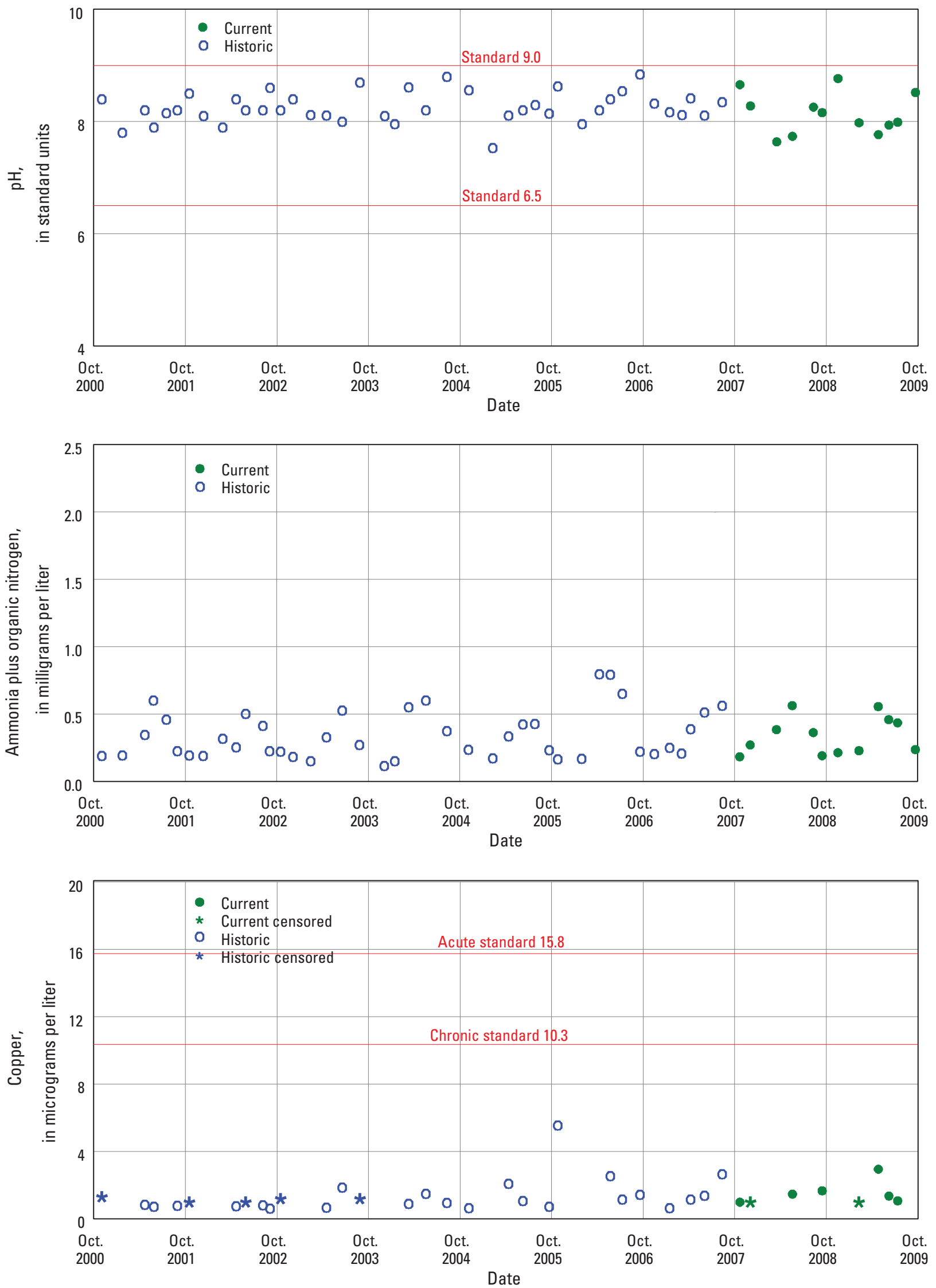

Figure 23. Time-series plots of selected water-quality constituents for Tomichi Creek at Gunnison, Colorado. 
Table 19. Summary of measured constituents and properties for Tomichi Creek at Gunnison, Colorado, station 09119000.

[--, no data or not applicable; L, low; M, Medium; H, High; NTRU, Nephelometric Turbidity Ratio Unit; E. coli, Escherichia coli; mg/m² , milligrams per square meter; USEPA, U.S. Environmental Protection Agency]

\begin{tabular}{|c|c|c|c|c|c|c|c|c|c|c|c|c|c|c|}
\hline $\begin{array}{c}\text { Property } \\
\text { or } \\
\text { constituent }\end{array}$ & $\begin{array}{l}\text { Period } \\
\text { (water } \\
\text { years) }\end{array}$ & $\begin{array}{l}\text { Number } \\
\text { of } \\
\text { samples }\end{array}$ & $\begin{array}{c}\text { Number } \\
\text { of } \\
\text { censored } \\
\text { values }\end{array}$ & Median $^{1}$ & Maximum & $\begin{array}{c}\text { Date } \\
\text { of } \\
\text { Maximum }\end{array}$ & $\begin{array}{c}\text { 15th } \\
\text { percentile }\end{array}$ & $\begin{array}{c}\text { 85th } \\
\text { percentile }\end{array}$ & $\begin{array}{c}\text { Geometric } \\
\text { Mean }^{2}\end{array}$ & $\begin{array}{l}\text { Standard } \\
\text { or } \\
\text { Chronic } \\
\text { standard }^{3}\end{array}$ & $\begin{array}{l}\text { Number of } \\
\text { exceedences } \\
\text { Standard or } \\
\text { Chronic }\end{array}$ & $\begin{array}{l}\text { Acute } \\
\text { standard }\end{array}$ & $\begin{array}{l}\text { Number of } \\
\text { exceedences } \\
\text { Acute }\end{array}$ & $\begin{array}{c}\text { Level } \\
\text { of } \\
\text { concern }\end{array}$ \\
\hline $\begin{array}{l}\text { Instantaneous discharge, in cubic feet } \\
\text { per second }\end{array}$ & $1995-2007$ & 82 & 0 & 102 & 2,370 & $06 / 19 / 95$ & 59 & 295 & -- & -- & -- & -- & - & -- \\
\hline $\begin{array}{l}\text { Instantaneous discharge, in cubic feet } \\
\text { per second }\end{array}$ & 2008-2009 & 12 & 0 & 100 & 697 & $05 / 19 / 08$ & 74 & 489 & -- & -- & -- & -- & -- & -- \\
\hline Turbidity, in NTRU & 2001-2007 & 28 & 0 & 3.5 & 13.6 & $05 / 29 / 01$ & 2.3 & 10.4 & -- & -- & -- & -- & -- & -- \\
\hline Turbidity, in NTRU & 2008-2009 & 8 & 0 & 5.2 & 24.7 & $04 / 27 / 09$ & 1.7 & 19.7 & -- & -- & -- & -- & -- & -- \\
\hline $\begin{array}{l}\text { Dissolved oxygen, in milligrams per } \\
\text { liter }\end{array}$ & 1995-2007 & 77 & 0 & 9.4 & 12.4 & $09 / 19 / 06$ & 7.9 & 10.9 & -- & 6.0 & 1 & -- & -- & $\mathrm{L}$ \\
\hline $\begin{array}{l}\text { Dissolved oxygen, in milligrams per } \\
\text { liter }\end{array}$ & $2008-2009$ & 12 & 0 & 10.2 & 13.3 & $11 / 17 / 08$ & 8.2 & 12.7 & -- & 6.0 & 0 & -- & -- & $\mathrm{L}$ \\
\hline $\mathrm{pH}$, in standard units & 1995-2007 & 77 & 0 & 8.2 & 8.8 & ${ }^{4} 09 / 19 / 06$ & 7.9 & 8.5 & -- & 6.5 & 0 & 9.0 & -- & $\mathrm{L}$ \\
\hline $\mathrm{pH}$, in standard units & 2008-2009 & 12 & 0 & 8.1 & 8.8 & $11 / 17 / 08$ & 7.7 & 8.7 & -- & 6.5 & 0 & 9.0 & -- & $\mathrm{L}$ \\
\hline $\begin{array}{l}\text { Specific conductance, in microsiemens } \\
\text { per centimeter }\end{array}$ & 1995-2007 & 76 & 0 & 262 & 399 & $05 / 31 / 02$ & 233 & 335 & -- & -- & -- & -- & -- & -- \\
\hline $\begin{array}{l}\text { Specific conductance, in microsiemens } \\
\text { per centimeter }\end{array}$ & $2008-2009$ & 12 & 0 & 257 & 350 & 07/13/09 & 214 & 316 & -- & -- & -- & -- & -- & -- \\
\hline Temperature, degrees Celsius & 1995-2007 & 77 & 0 & 10.3 & 21.5 & 07/18/01 & 0.1 & 18.0 & -- & 17.0 & 12 & -- & -- & $\mathrm{H}$ \\
\hline Temperature, degrees Celsius & $2008-2009$ & 12 & 0 & 10.3 & 19.7 & $08 / 11 / 08$ & 0.7 & 19.0 & -- & 17.0 & 2 & -- & -- & $\mathrm{H}$ \\
\hline Calcium, in milligrams per liter & $1995-2007$ & 51 & 0 & 33.6 & 56.7 & $05 / 31 / 02$ & 28.0 & 47.1 & -- & -- & -- & -- & -- & -- \\
\hline Calcium, in milligrams per liter & $2008-2009$ & 8 & 0 & 33.8 & 46.8 & 07/13/09 & 23.6 & 42.6 & -- & -- & -- & -- & -- & -- \\
\hline Magnesium, in milligrams per liter & $1995-2007$ & 51 & 0 & 8.72 & 14.1 & 07/11/06 & 7.64 & 12.0 & -- & -- & -- & -- & -- & -- \\
\hline Magnesium, in milligrams per liter & 2008-2009 & 8 & 0 & 8.51 & 11.8 & 07/13/09 & 6.45 & 10.8 & -- & -- & -- & -- & -- & -- \\
\hline $\begin{array}{l}\text { Ammonia plus organic nitrogen, in } \\
\text { milligrams per liter }\end{array}$ & 1995-2007 & 77 & 6 & 0.28 & 1.2 & $04 / 07 / 00$ & 0.15 & 0.56 & -- & -- & -- & -- & -- & -- \\
\hline $\begin{array}{l}\text { Ammonia plus organic nitrogen, in } \\
\text { milligrams per liter }\end{array}$ & 2008-2009 & 12 & 0 & 0.32 & 0.56 & $05 / 19 / 08$ & 0.19 & 0.56 & -- & -- & -- & -- & -- & -- \\
\hline $\begin{array}{l}\text { Ammonia nitrogen, in milligrams per } \\
\text { liter }\end{array}$ & 1995-2007 & 77 & 50 & 5 & 0.050 & ${ }^{4} 04 / 01 / 96$ & 5 & 0.015 & -- & 1.84 & 0 & 4.42 & 0 & $\mathrm{~L}$ \\
\hline $\begin{array}{l}\text { Ammonia nitrogen, in milligrams per } \\
\text { liter }\end{array}$ & $2008-2009$ & 12 & 10 & 5 & 0.023 & 02/09/09 & 5 & 0.016 & -- & 2.04 & 0 & 5.15 & 0 & $\mathrm{~L}$ \\
\hline $\begin{array}{l}\text { Nitrite plus nitrate nitrogen, in mil- } \\
\text { ligrams per liter }\end{array}$ & 1995-2007 & 77 & 44 & 5 & 0.114 & $01 / 31 / 06$ & 5 & 0.065 & -- & 10.0 & 0 & -- & -- & $\mathrm{L}$ \\
\hline $\begin{array}{l}\text { Nitrite plus nitrate nitrogen, in mil- } \\
\text { ligrams per liter }\end{array}$ & $2008-2009$ & 12 & 4 & 0.015 & 0.097 & 02/09/09 & 5 & 0.045 & -- & 10.0 & 0 & -- & -- & $\mathrm{L}$ \\
\hline Nitrite nitrogen, in milligrams per liter & 1995-2007 & 77 & 53 & 5 & 0.020 & ${ }^{4} 04 / 01 / 96$ & 5 & 0.001 & -- & 0.05 & 0 & -- & -- & $\mathrm{L}$ \\
\hline Nitrite nitrogen, in milligrams per liter & 2008-2009 & 12 & 6 & 0.001 & ${ }^{6}<0.002$ & -- & 5 & 5 & -- & 0.05 & 0 & -- & -- & $\mathrm{L}$ \\
\hline
\end{tabular}


[--, no data or not applicable; L, low; M, Medium; H, High; NTRU, Nephelometric Turbidity Ratio Unit; E. coli, Escherichia coli; mg/m², milligrams per square meter; USEPA, U.S. Environmental Protection Agency]

\begin{tabular}{|c|c|c|c|c|c|c|c|c|c|c|c|c|c|c|}
\hline $\begin{array}{c}\text { Property } \\
\text { or } \\
\text { constituent }\end{array}$ & $\begin{array}{l}\text { Period } \\
\text { (water } \\
\text { years) }\end{array}$ & $\begin{array}{c}\text { Number } \\
\text { of } \\
\text { samples }\end{array}$ & $\begin{array}{l}\text { Number } \\
\text { of } \\
\text { censored } \\
\text { values }\end{array}$ & Median' & Maximum & $\begin{array}{c}\text { Date } \\
\text { of } \\
\text { Maximum }\end{array}$ & $\begin{array}{c}\text { 15th } \\
\text { percentile }\end{array}$ & $\begin{array}{c}\text { 85th } \\
\text { percentile }\end{array}$ & $\begin{array}{l}\text { Geometric } \\
\text { Mean }^{2}\end{array}$ & $\begin{array}{l}\text { Standard } \\
\text { or } \\
\text { Chronic } \\
\text { standard }^{3}\end{array}$ & $\begin{array}{l}\text { Number of } \\
\text { exceedences } \\
\text { Standard or } \\
\text { Chronic }\end{array}$ & $\begin{array}{l}\text { Acute } \\
\text { standard }\end{array}$ & $\begin{array}{c}\text { Number of } \\
\text { exceedences } \\
\text { Acute }\end{array}$ & $\begin{array}{c}\text { Level } \\
\text { of } \\
\text { concern }\end{array}$ \\
\hline Orthophosphate, in milligrams per liter & 1995-2007 & 77 & 4 & 0.020 & 0.047 & $08 / 13 / 07$ & 0.008 & 0.030 & -- & -- & -- & -- & -- & -- \\
\hline Orthophosphate, in milligrams per liter & 2008-2009 & 12 & 0 & 0.020 & 0.027 & $08 / 11 / 08$ & 0.015 & 0.025 & -- & -- & -- & -- & -- & -- \\
\hline $\begin{array}{l}\text { Phosphorus (total), in milligrams per } \\
\text { liter }\end{array}$ & 1995-2007 & 77 & 1 & 0.048 & 0.269 & $04 / 07 / 00$ & 0.026 & 0.078 & -- & -- & -- & -- & -- & -- \\
\hline $\begin{array}{l}\text { Phosphorus (total), in milligrams per } \\
\text { liter }\end{array}$ & 2008-2009 & 12 & 0 & 0.053 & 0.116 & $04 / 27 / 09$ & 0.037 & 0.116 & -- & -- & -- & -- & -- & -- \\
\hline E. coli, in colonies per 100 milliliter & 2001-2007 & 41 & 3 & 23 & 330 & $05 / 24 / 06$ & 2 & 7 & 16.7 & 126 & 5 & -- & -- & $\mathrm{L}$ \\
\hline E. coli, in colonies per 100 milliliter & 2008-2009 & 11 & 1 & 14 & 140 & $05 / 19 / 08$ & 1 & 7 & -- & 126 & 1 & -- & -- & $\mathrm{L}$ \\
\hline $\begin{array}{l}\text { Biomass periphyton, ashfree drymass, } \\
\text { in grams per square meter }\end{array}$ & 1998-2007 & 9 & 0 & 29.5 & 43.0 & $08 / 28 / 03$ & 11.3 & 40.4 & -- & -- & -- & -- & -- & -- \\
\hline $\begin{array}{l}\text { Biomass periphyton, ashfree drymass, } \\
\text { in grams per square meter }\end{array}$ & 2008-2009 & 2 & 1 & 5.5 & 11.0 & $09 / 22 / 09$ & 5 & 11.0 & -- & -- & -- & -- & -- & -- \\
\hline $\begin{array}{l}\text { Periphyton, biomass, ash weight, in } \\
\text { grams per square meter }\end{array}$ & 1998-2007 & 9 & 0 & 713 & 1,796 & $08 / 28 / 03$ & 204 & 1,448 & -- & -- & -- & -- & -- & -- \\
\hline $\begin{array}{l}\text { Periphyton, biomass, ash weight, in } \\
\text { grams per square meter }\end{array}$ & 2008-2009 & 2 & 0 & 338 & 340 & 09/15/08 & 337 & 340 & -- & -- & -- & -- & -- & -- \\
\hline $\begin{array}{l}\text { Periphyton, biomass, dry weight, in } \\
\text { grams per square meter }\end{array}$ & 1998-2007 & 9 & 0 & 742 & 1,838 & $08 / 28 / 03$ & 219 & 1,486 & -- & -- & -- & -- & -- & -- \\
\hline $\begin{array}{l}\text { Periphyton, biomass, dry weight, in } \\
\text { grams per square meter }\end{array}$ & 2008-2009 & 2 & 0 & 348 & 348 & $09 / 22 / 09$ & 347 & 348 & -- & -- & -- & -- & -- & -- \\
\hline $\begin{array}{l}\text { Chlorophyll a, periphyton, chromo- } \\
\text { fluoro, in } \mathrm{mg} / \mathrm{m}^{2}\end{array}$ & 1998-2007 & 10 & 0 & 27.3 & 115 & $07 / 11 / 06$ & 3.06 & 92.7 & -- & -- & -- & -- & -- & -- \\
\hline $\begin{array}{l}\text { Chlorophyll a, periphyton, chromo- } \\
\text { fluoro, in } \mathrm{mg} / \mathrm{m}^{2}\end{array}$ & 2008-2009 & 2 & 0 & 32.5 & 33.9 & 09/22/09 & 31.2 & 33.9 & -- & -- & -- & -- & -- & -- \\
\hline Pheophytin a, periphyton, in $\mathrm{mg} / \mathrm{m}^{2}$ & 2001-2007 & 8 & 0 & 12.2 & 34.3 & $10 / 18 / 01$ & 2.2 & 31.7 & -- & -- & -- & -- & -- & -- \\
\hline Pheophytin a, periphyton, in $\mathrm{mg} / \mathrm{m}^{2}$ & 2008-2009 & 2 & 0 & 11.3 & 11.4 & 09/15/08 & 11.1 & 11.4 & -- & -- & -- & -- & -- & -- \\
\hline Aluminum, in micrograms per liter & 1995-2007 & 44 & 23 & 5 & 80.0 & $06 / 19 / 95$ & 5 & 3.5 & -- & -- & -- & -- & -- & -- \\
\hline Aluminum, in micrograms per liter & 2008-2009 & 8 & 0 & 3.0 & 33.9 & $04 / 27 / 09$ & 2.0 & 26.5 & -- & -- & -- & -- & -- & -- \\
\hline Cadmium, in micrograms per liter & 1995-2007 & 44 & 42 & 5 & ${ }^{6}<1$ & -- & 5 & 5 & -- & 0.48 & 0 & 1.97 & 0 & $\mathrm{~L}$ \\
\hline Cadmium, in micrograms per liter & $2008-2009$ & 8 & 4 & 0.01 & 0.02 & $04 / 27 / 09$ & 5 & 0.02 & -- & 0.48 & 0 & 1.97 & 0 & $\mathrm{~L}$ \\
\hline Copper, in micrograms per liter & 1995-2007 & 44 & 15 & 0.77 & 5.5 & $10 / 27 / 05$ & 5 & 1.9 & -- & 10.3 & 0 & 15.8 & 0 & $\mathrm{~L}$ \\
\hline Copper, in micrograms per liter & 2008-2009 & 8 & 2 & 1.2 & 3.0 & 04/27/09 & 5 & 2.5 & -- & 10.3 & 0 & 15.8 & 0 & $\mathrm{~L}$ \\
\hline Iron, in micrograms per liter & $1995-2007$ & 39 & 0 & 64 & 190 & $06 / 19 / 95$ & 34 & 110 & -- & 300 & 0 & -- & -- & $\mathrm{L}$ \\
\hline Iron, in micrograms per liter & 2008-2009 & 8 & 0 & 88 & 140 & 06/08/09 & 53 & 126 & -- & 300 & 0 & -- & -- & $\mathrm{L}$ \\
\hline Lead, in micrograms per liter & 1995-2007 & 44 & 29 & 5 & 0.29 & 09/19/06 & 5 & 0.12 & -- & 3.02 & 0 & 77.6 & 0 & $\mathrm{~L}$ \\
\hline Lead, in micrograms per liter & 2008-2009 & 8 & 0 & 0.17 & 0.38 & 04/27/09 & 0.06 & 0.38 & -- & 3.02 & 0 & 77.6 & 0 & $\mathrm{~L}$ \\
\hline
\end{tabular}


Table 19. Summary of measured constituents and properties for Tomichi Creek at Gunnison, Colorado, station 09119000.—Continued

[--, no data or not applicable; L, low; M, Medium; H, High; NTRU, Nephelometric Turbidity Ratio Unit; E. coli, Escherichia coli; mg/m², milligrams per square meter; USEPA, U.S. Environmental Protection Agency]

\begin{tabular}{|c|c|c|c|c|c|c|c|c|c|c|c|c|c|c|}
\hline $\begin{array}{l}\text { Property } \\
\text { or } \\
\text { constituent }\end{array}$ & $\begin{array}{l}\text { Period } \\
\text { (water } \\
\text { years) }\end{array}$ & $\begin{array}{c}\text { Number } \\
\text { of } \\
\text { samples }\end{array}$ & $\begin{array}{c}\text { Number } \\
\text { of } \\
\text { censored } \\
\text { values }\end{array}$ & Median $^{1}$ & Maximum & $\begin{array}{c}\text { Date } \\
\text { of } \\
\text { Maximum }\end{array}$ & $\begin{array}{c}\text { 15th } \\
\text { percentile }\end{array}$ & $\begin{array}{c}\text { 85th } \\
\text { percentile }\end{array}$ & $\begin{array}{c}\text { Geometric } \\
\text { Mean }^{2}\end{array}$ & $\begin{array}{c}\text { Standard } \\
\text { or } \\
\text { Chronic } \\
\text { standard }^{3}\end{array}$ & $\begin{array}{l}\text { Number of } \\
\text { exceedences } \\
\text { Standard or } \\
\text { Chronic }\end{array}$ & $\begin{array}{l}\text { Acute } \\
\text { standard }\end{array}$ & $\begin{array}{l}\text { Number of } \\
\text { exceedences } \\
\text { Acute }\end{array}$ & $\begin{array}{c}\text { Level } \\
\text { of } \\
\text { concern }\end{array}$ \\
\hline Manganese, in micrograms per liter & 1995-2007 & 39 & 0 & 31.8 & 124 & $04 / 22 / 02$ & 15.6 & 73.2 & -- & 1,745 & 0 & 3,159 & 0 & $\mathrm{~L}$ \\
\hline Manganese, in micrograms per liter & 2008-2009 & 8 & 0 & 31.4 & 55.2 & 06/08/09 & 18.6 & 49.8 & -- & 1,745 & 0 & 3,159 & 0 & $\mathrm{~L}$ \\
\hline Silver, in micrograms per liter & 1999-2007 & 32 & 32 & 5 & ${ }^{6}<0.3$ & -- & 5 & 5 & -- & 0.10 & 0 & 2.71 & 0 & $\mathrm{~L}$ \\
\hline Silver, in micrograms per liter & 2008-2009 & 8 & 6 & 5 & ${ }^{6}<0.1$ & -- & 5 & 5 & -- & 0.10 & 0 & 2.71 & 0 & $\mathrm{~L}$ \\
\hline Zinc, in micrograms per liter & 1995-2007 & 44 & 25 & 5 & 11.5 & $04 / 23 / 01$ & 5 & 3.0 & -- & 153 & 0 & 166 & 0 & $\mathrm{~L}$ \\
\hline Zinc, in micrograms per liter & 2008-2009 & 8 & 1 & 2.7 & 6.6 & $04 / 27 / 09$ & 0.38 & 5.4 & -- & 153 & 0 & 166 & 0 & $\mathrm{~L}$ \\
\hline $\begin{array}{l}\text { Suspended sediment, in milligrams } \\
\text { per liter }\end{array}$ & 1995-2007 & 49 & 0 & 8.8 & 135 & $03 / 27 / 97$ & 3.1 & 29.0 & -- & -- & -- & -- & -- & -- \\
\hline $\begin{array}{l}\text { Suspended sediment, in milligrams } \\
\text { per liter }\end{array}$ & 2008-2009 & 8 & 0 & 9.0 & 55.0 & $04 / 27 / 09$ & 1.4 & 41.0 & -- & -- & -- & -- & -- & -- \\
\hline
\end{tabular}

${ }^{1}$ Censored values were replaced with 0 to compute median and 85 th percentiles (coliform censored values replaced with 1 ) see definitions section.

${ }^{2}$ Geometric mean listed for E. coli. Geometric mean computed for combined period of record (2001-2009).

${ }^{3}$ Colorado Department of Public Health and Environment, classification and numeric standards for Gunnison and Lower Dolores River Basins (2010a); and USEPA National Recommended Water Quality Criteria (2009). ${ }^{4}$ Multiple dates for maximum.

${ }^{5}$ Median values and percentiles were not tabled when they were calculated as zero.

${ }^{6} \mathrm{All}$ values below laboratory reporting level (LRL), current LRL used for current period maximum, highest LRL used for historic period.

${ }^{7}$ Use geometric mean for comparison to standard. 
Table 20. Summary of seasonal Kendall trend tests for Tomichi Creek at Gunnison, Colorado, station 09119000.

[E. coli, Escherichia coli]

\begin{tabular}{lcccc}
\hline \multicolumn{1}{c}{ Property or constituent } & $\begin{array}{c}\text { Period } \\
\text { (water years) }\end{array}$ & Kendall tau $(\tau)$ & p-Value & Trend direction \\
\hline Specific conductance, in microsiemens per centimeter & $1995-2009$ & 0.191 & 0.055 & None \\
pH, in standard units & $1995-2009$ & 0.207 & 0.038 & Up \\
Ammonia plus organic nitrogen, in milligrams per liter & $1999-2009$ & 0.277 & 0.019 & Up \\
Phosphorus (total), in milligrams per liter & $2000-2009$ & 0.056 & 0.687 & None \\
Orthophosphate, in milligrams per liter & $2000-2009$ & 0.028 & 0.089 & None \\
Calcium, in milligrams per liter & $2001-2009$ & -0.289 & 0.454 & None \\
Magnesium, in milligrams per liter & $2001-2009$ & -0.133 & 0.003 & Up \\
Copper, in micrograms per liter & $2001-2009$ & 0.500 & 0.522 & None \\
Iron, in micrograms per liter & $1995-2009$ & 0.094 & 0.082 & 0.069 \\
Manganese, in micrograms per liter & $1995-2009$ & -0.215 & -0.132 & None \\
Suspended sediment, in milligrams per liter & $1995-2009$ & & 0.354 \\
E. coli, in colonies per 100 milliliter & $2001-2009$ & & None \\
\hline
\end{tabular}




\section{Gunnison River at County Road 32 below Gunnison, Colorado}

Current Reason for Inclusion: This station is downstream from the City of Gunnison wastewater treatment plant discharge. When compared to sites Gunnison River at Gunnison and Tomichi Creek at Gunnison, this site should give insight to the change in water quality due to point and nonpoint sources in the City of Gunnison. Characterize water-quality conditions upstream from Curecanti National Recreation area. Long-term monitoring.

General Station Information:

Location:

County Road 32 bridge, 0.25 mi south of U.S. Highway 50, and 3.3 mi west of Gunnison.

Station Type:

USGS water quality

Period of Record:

Water quality: December 1994-September 2009

Latitude: 383103

Drainage area: $2,128 \mathrm{mi}^{2}$

HUC: 14020002

Longitude: 1065942

Stream segment: 14

USGS Data Summary: Water Years 2008-2009:

General Chemistry:

pH: Low concern

Dissolved oxygen: Low concern

Temperature: Low concern

Nutrients:

Low concern

E. coli:

Low concern

Trace Elements/metals:

Low concern

Other constituents of concern: $\quad$ None for constituents tested.

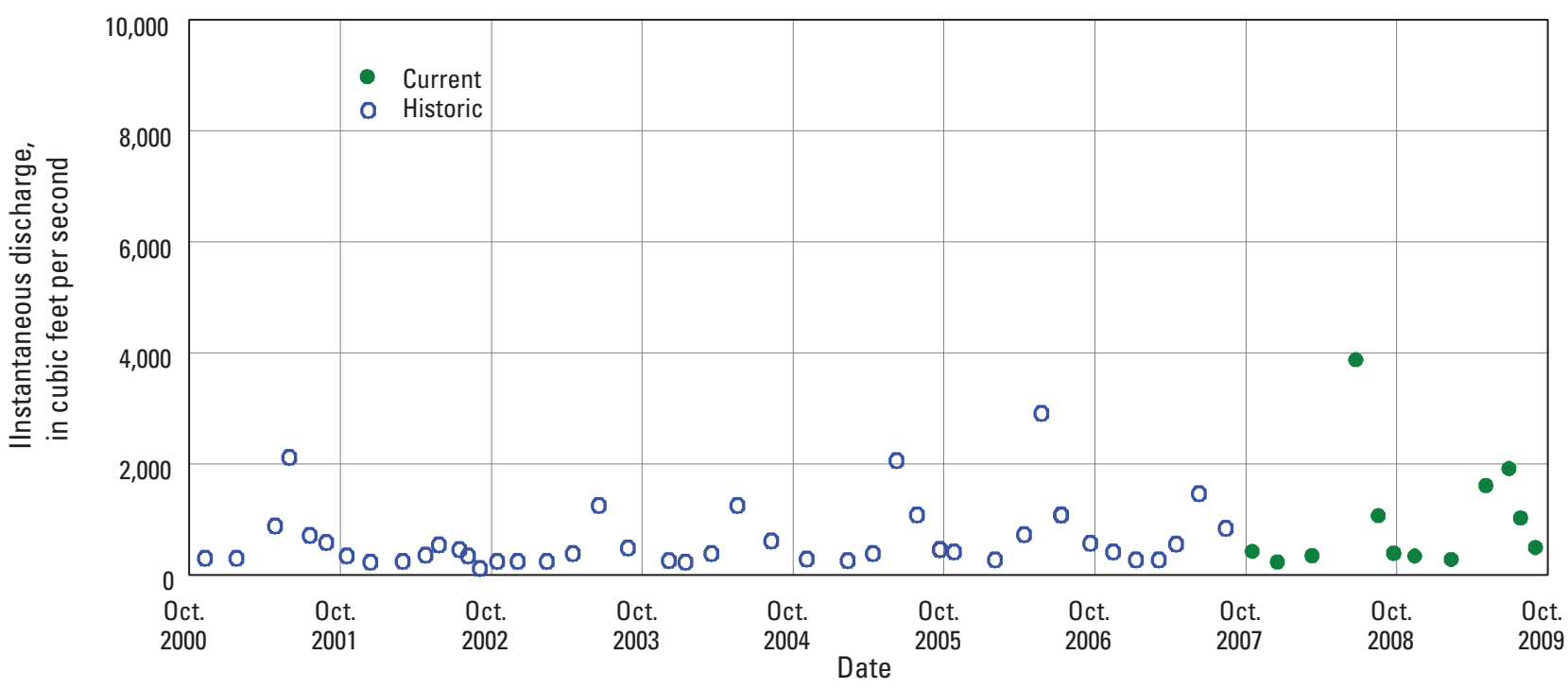

Figure 24. Time distribution and associated discharge of water-quality samples for Gunnison River at County Road 32 below Gunnison, Colorado. 

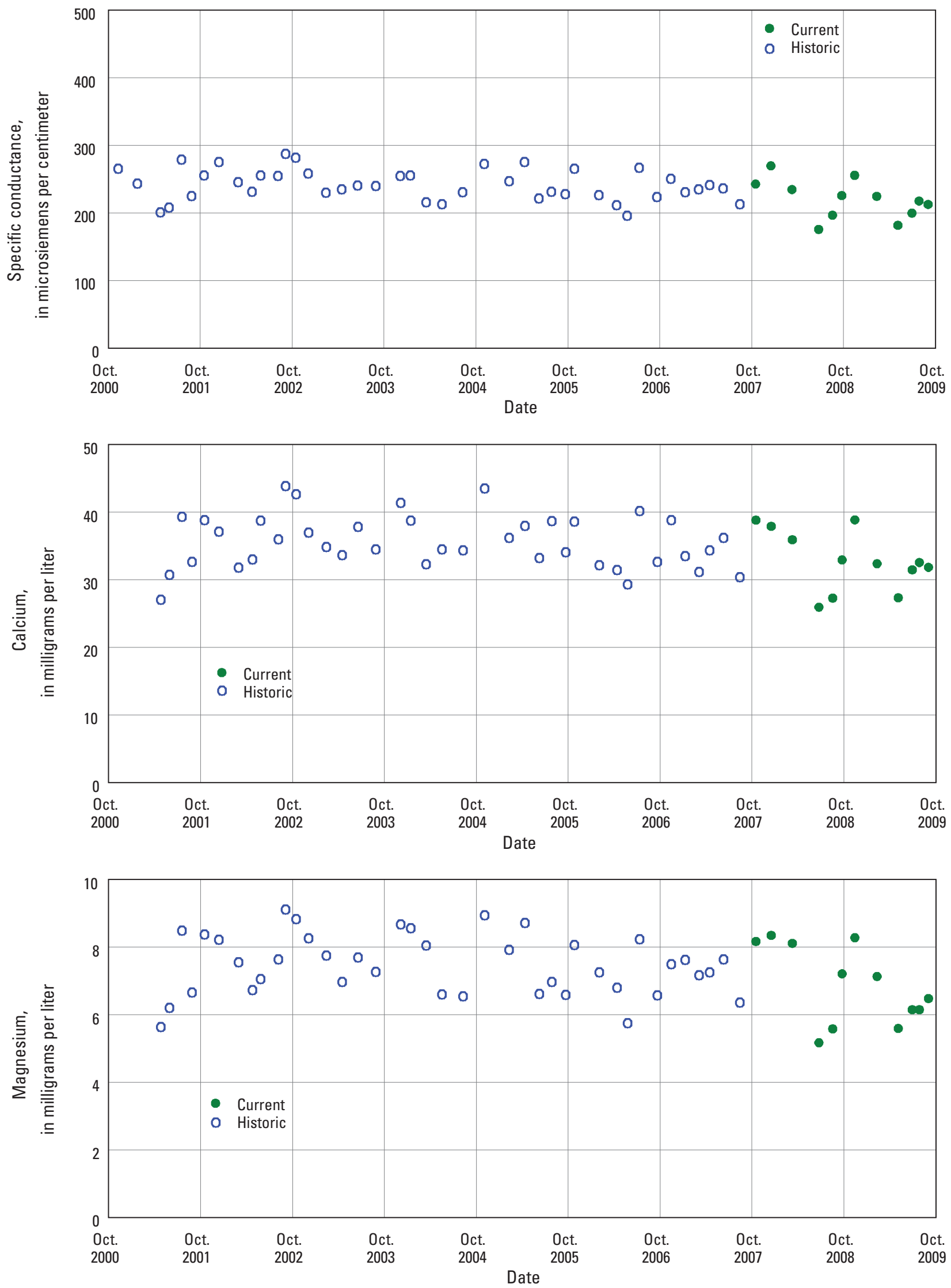

Figure 25. Time-series plots of selected water-quality constituents for Gunnison River at County Road 32 below Gunnison, Colorado. 
Table 21. Summary of measured constituents and properties for Gunnison River at CR 32 below Gunnison, Colorado, station 383103106594200.

[--, no data or not applicable; L, low; M, Medium; H, High; NTRU, Nephelometric Turbidity Ratio Unit; E. coli, Escherichia coli; mg/m² milligrams per square meter; USEPA, U.S. Environmental Protection Agency]

\begin{tabular}{|c|c|c|c|c|c|c|c|c|c|c|c|c|c|c|}
\hline $\begin{array}{l}\text { Property } \\
\text { or } \\
\text { constituent }\end{array}$ & $\begin{array}{l}\text { Period } \\
\text { (water } \\
\text { years) }\end{array}$ & $\begin{array}{c}\text { Number } \\
\text { of } \\
\text { samples }\end{array}$ & $\begin{array}{c}\text { Number } \\
\text { of } \\
\text { censored } \\
\text { values }\end{array}$ & Median $^{1}$ & Maximum & $\begin{array}{c}\text { Date } \\
\text { of } \\
\text { Maximum }\end{array}$ & $\begin{array}{c}\text { 15th } \\
\text { percentile }\end{array}$ & $\begin{array}{c}\text { 85th } \\
\text { percentile }\end{array}$ & $\begin{array}{c}\text { Geometric } \\
\text { Mean }^{2}\end{array}$ & $\begin{array}{c}\text { Standard } \\
\text { or } \\
\text { Chronic } \\
\text { standard }^{3}\end{array}$ & $\begin{array}{c}\text { Number of } \\
\text { exceedences } \\
\text { Standard or } \\
\text { Chronic } \\
\end{array}$ & $\begin{array}{c}\text { Acute } \\
\text { standard }\end{array}$ & $\begin{array}{c}\begin{array}{c}\text { Number } \\
\text { of } \\
\text { exceedences } \\
\text { Acute }\end{array} \\
\end{array}$ & $\begin{array}{c}\text { Level } \\
\text { of } \\
\text { concern }\end{array}$ \\
\hline $\begin{array}{l}\text { Instantaneous discharge, in cubic feet } \\
\text { per second }\end{array}$ & 1995-2007 & 102 & 0 & 605 & 7,550 & $06 / 16 / 95$ & 293 & 2,230 & -- & -- & -- & -- & -- & -- \\
\hline $\begin{array}{l}\text { Instantaneous discharge, in cubic feet } \\
\text { per second }\end{array}$ & 2008-2009 & 13 & 0 & 429 & 3,880 & $06 / 24 / 08$ & 291 & 1,890 & -- & -- & -- & -- & -- & -- \\
\hline Turbidity, in NTRU & $2001-2007$ & 40 & 9 & 2.8 & 13.7 & 04/27/01 & 0.0 & 6.7 & -- & -- & -- & -- & -- & -- \\
\hline Turbidity, in NTRU & $2008-2009$ & 12 & 2 & 2.2 & 10.2 & $05 / 05 / 09$ & 0.0 & 7.2 & -- & -- & -- & -- & -- & -- \\
\hline $\begin{array}{l}\text { Dissolved oxygen, in milligrams per } \\
\text { liter }\end{array}$ & 1995-2007 & 100 & 0 & 9.4 & 13.0 & $01 / 10 / 07$ & 8.3 & 11.2 & -- & 6.0 & 0 & -- & -- & $\mathrm{L}$ \\
\hline $\begin{array}{l}\text { Dissolved oxygen, in milligrams per } \\
\text { liter }\end{array}$ & 2008-2009 & 12 & 0 & 9.0 & 12.5 & $12 / 17 / 07$ & 8.1 & 11.1 & -- & 6.0 & 0 & -- & -- & $\mathrm{L}$ \\
\hline $\mathrm{pH}$, in standard units & 1995-2007 & 100 & 0 & 8.2 & 8.8 & $02 / 10 / 05$ & 8.0 & 8.5 & -- & 6.5 & 0 & 9.0 & -- & $\mathrm{L}$ \\
\hline $\mathrm{pH}$, in standard units & 2008-2009 & 12 & 0 & 8.2 & 8.6 & $09 / 24 / 08$ & 7.8 & 8.5 & -- & 6.5 & 0 & 9.0 & -- & $\mathrm{L}$ \\
\hline $\begin{array}{l}\text { Specific conductance, in microsiemens } \\
\text { per centimeter }\end{array}$ & 1995-2007 & 100 & 0 & 226 & 288 & $09 / 05 / 02$ & 191 & 254 & -- & -- & -- & -- & -- & -- \\
\hline $\begin{array}{l}\text { Specific conductance, in microsiemens } \\
\text { per centimeter }\end{array}$ & 2008-2009 & 12 & 0 & 222 & 270 & $12 / 17 / 07$ & 182 & 257 & -- & -- & -- & -- & -- & -- \\
\hline Temperature, degrees Celsius & 1995-2007 & 100 & 0 & 7.6 & 17.3 & $07 / 20 / 98$ & 0.0 & 14.4 & -- & 17.0 & 1 & -- & -- & $\mathrm{L}$ \\
\hline Temperature, degrees Celsius & 2008-2009 & 12 & 0 & 10.3 & 17.1 & $07 / 28 / 09$ & 0.0 & 14.6 & -- & 17.0 & 1 & -- & -- & $\mathrm{L}$ \\
\hline Calcium, in milligrams per liter & 1995-2007 & 89 & 0 & 31.1 & 43.9 & ${ }^{4} 09 / 05 / 02$ & 26.0 & 37.9 & -- & -- & -- & -- & -- & -- \\
\hline Calcium, in milligrams per liter & 2008-2009 & 12 & 0 & 32.5 & 38.9 & $11 / 14 / 08$ & 27.3 & 38.9 & -- & -- & -- & -- & -- & -- \\
\hline Magnesium, in milligrams per liter & 1995-2007 & 89 & 0 & 6.73 & 9.12 & $09 / 05 / 02$ & 5.58 & 7.99 & -- & -- & -- & -- & -- & -- \\
\hline Magnesium, in milligrams per liter & $2008-2009$ & 12 & 0 & 6.81 & 8.36 & $12 / 17 / 07$ & 5.57 & 8.29 & -- & -- & -- & -- & -- & -- \\
\hline $\begin{array}{l}\text { Ammonia plus organic nitrogen, in } \\
\text { milligrams per liter }\end{array}$ & 1995-2007 & 100 & 19 & 0.19 & 0.60 & ${ }^{4} 06 / 16 / 95$ & 5 & 0.35 & -- & -- & -- & -- & -- & -- \\
\hline $\begin{array}{l}\text { Ammonia plus organic nitrogen, in } \\
\text { milligrams per liter }\end{array}$ & 2008-2009 & 12 & 0 & 0.15 & 0.31 & 05/05/09 & 0.10 & 0.24 & -- & -- & -- & -- & -- & -- \\
\hline $\begin{array}{l}\text { Ammonia nitrogen, in milligrams } \\
\text { per liter }\end{array}$ & 1995-2007 & 99 & 60 & 5 & 0.069 & $05 / 22 / 98$ & 5 & 0.020 & -- & 1.80 & 0 & 4.01 & 0 & $\mathrm{~L}$ \\
\hline $\begin{array}{l}\text { Ammonia nitrogen, in milligrams } \\
\text { per liter }\end{array}$ & 2008-2009 & 12 & 9 & 5 & 0.016 & $10 / 17 / 07$ & 5 & 0.015 & -- & 1.85 & 0 & 4.26 & 0 & $\mathrm{~L}$ \\
\hline $\begin{array}{l}\text { Nitrite plus nitrate nitrogen, in mil- } \\
\text { ligrams per liter }\end{array}$ & 1995-2007 & 99 & 13 & 0.059 & 0.192 & $11 / 15 / 06$ & 0.013 & 0.106 & -- & 10.0 & 0 & -- & -- & $\mathrm{L}$ \\
\hline $\begin{array}{l}\text { Nitrite plus nitrate nitrogen, in mil- } \\
\text { ligrams per liter }\end{array}$ & 2008-2009 & 12 & 2 & 0.056 & 0.173 & $12 / 17 / 07$ & 5 & 0.115 & -- & 10.0 & 0 & -- & -- & $\mathrm{L}$ \\
\hline Nitrite nitrogen, in milligrams per liter & 1995-2007 & 99 & 56 & 5 & 0.020 & ${ }^{4} 06 / 13 / 96$ & 5 & 0.002 & -- & 0.05 & 0 & -- & -- & $\mathrm{L}$ \\
\hline Nitrite nitrogen, in milligrams per liter & $2008-2009$ & 12 & 7 & 5 & 0.002 & $10 / 17 / 07$ & 5 & 0.002 & -- & 0.05 & 0 & -- & -- & $\mathrm{L}$ \\
\hline
\end{tabular}


Table 21. Summary of measured constituents and properties for Gunnison River at CR 32 below Gunnison, Colorado, station 383103106594200.-Continued

[--, no data or not applicable; L, low; M, Medium; H, High; NTRU, Nephelometric Turbidity Ratio Unit; E. coli, Escherichia coli; mg/m² milligrams per square meter; USEPA, U.S. Environmental Protection Agency]

\begin{tabular}{|c|c|c|c|c|c|c|c|c|c|c|c|c|c|c|}
\hline $\begin{array}{l}\text { Property } \\
\text { or } \\
\text { constituent }\end{array}$ & $\begin{array}{l}\text { Period } \\
\text { (water } \\
\text { years) }\end{array}$ & $\begin{array}{c}\text { Number } \\
\text { of } \\
\text { samples }\end{array}$ & $\begin{array}{c}\begin{array}{c}\text { Number } \\
\text { of }\end{array} \\
\text { censored } \\
\text { values }\end{array}$ & Median' & Maximum & $\begin{array}{c}\text { Date } \\
\text { of } \\
\text { Maximum }\end{array}$ & $\begin{array}{c}\text { 15th } \\
\text { percentile }\end{array}$ & $\begin{array}{c}\text { 85th } \\
\text { percentile }\end{array}$ & $\begin{array}{c}\text { Geometric } \\
\text { Mean }^{2}\end{array}$ & $\begin{array}{c}\text { Standard } \\
\text { or } \\
\text { Chronic } \\
\text { standard }^{3}\end{array}$ & $\begin{array}{l}\text { Number of } \\
\text { exceedences } \\
\text { Standard or } \\
\text { Chronic }\end{array}$ & $\begin{array}{l}\text { Acute } \\
\text { standard }\end{array}$ & $\begin{array}{c}\begin{array}{c}\text { Number } \\
\text { of } \\
\text { exceedences } \\
\text { Acute }\end{array} \\
\end{array}$ & $\begin{array}{c}\text { Level } \\
\text { of } \\
\text { concern }\end{array}$ \\
\hline Orthophosphate, in milligrams per liter & 1995-2007 & 99 & 14 & 0.011 & 0.032 & $02 / 20 / 98$ & 0.003 & 0.020 & -- & -- & -- & -- & -- & -- \\
\hline Orthophosphate, in milligrams per liter & $2008-2009$ & 12 & 0 & 0.014 & 0.023 & $12 / 17 / 07$ & 0.006 & 0.019 & -- & -- & -- & -- & -- & -- \\
\hline $\begin{array}{l}\text { Phosphorus (total), in milligrams per } \\
\text { liter }\end{array}$ & $1995-2007$ & 100 & 7 & 0.032 & 0.170 & $06 / 16 / 95$ & 0.015 & 0.070 & -- & -- & -- & -- & -- & -- \\
\hline $\begin{array}{l}\text { Phosphorus (total), in milligrams } \\
\text { per liter }\end{array}$ & 2008-2009 & 12 & 0 & 0.031 & 0.050 & 05/05/09 & 0.021 & 0.048 & -- & -- & -- & -- & -- & -- \\
\hline E. coli, in colonies per 100 milliliter & 2001-2007 & 40 & 4 & 11 & 120 & 07/12/06 & 1 & 6 & 8.3 & 126 & 0 & -- & -- & $\mathrm{L}$ \\
\hline E. coli, in colonies per 100 milliliter & $2008-2009$ & 8 & 1 & 8 & 160 & 06/30/09 & 1 & 6 & -- & 126 & 1 & -- & -- & $\mathrm{L}$ \\
\hline $\begin{array}{l}\text { Biomass periphyton, ashfree drymass, } \\
\text { in grams per square meter }\end{array}$ & 2003-2007 & 5 & 2 & 13.8 & 24.7 & 08/10/04 & 5 & 24.7 & -- & -- & -- & -- & -- & -- \\
\hline $\begin{array}{l}\text { Biomass periphyton, ashfree drymass, } \\
\text { in grams per square meter }\end{array}$ & 2008-2009 & 2 & 0 & 16.0 & 18.2 & 09/02/09 & 13.7 & 18.2 & -- & -- & -- & -- & -- & -- \\
\hline $\begin{array}{l}\text { Periphyton, biomass, ash weight, in } \\
\text { grams per square meter }\end{array}$ & 2003-2007 & 5 & 0 & 540 & 908 & $09 / 22 / 05$ & 315 & 908 & -- & -- & -- & -- & -- & -- \\
\hline $\begin{array}{l}\text { Periphyton, biomass, ash weight, in } \\
\text { grams per square meter }\end{array}$ & 2008-2009 & 2 & 0 & 457 & 608 & 09/02/09 & 306 & 608 & -- & -- & -- & -- & -- & -- \\
\hline $\begin{array}{l}\text { Periphyton, biomass, dry weight, in } \\
\text { grams per square meter }\end{array}$ & 2003-2007 & 5 & 0 & 565 & 926 & $09 / 22 / 05$ & 328 & 926 & -- & -- & -- & -- & -- & -- \\
\hline $\begin{array}{l}\text { Periphyton, biomass, dry weight, in } \\
\text { grams per square meter }\end{array}$ & 2008-2009 & 2 & 0 & 473 & 626 & 09/02/09 & 320 & 626 & -- & -- & -- & -- & -- & -- \\
\hline $\begin{array}{l}\text { Chlorophyll a, periphyton, chromo- } \\
\text { fluoro, in } \mathrm{mg} / \mathrm{m}^{2}\end{array}$ & $2003-2007$ & 5 & 0 & 24.5 & 27.5 & $09 / 22 / 05$ & 13.3 & 27.5 & -- & -- & -- & -- & -- & -- \\
\hline $\begin{array}{l}\text { Chlorophyll a, periphyton, chromo- } \\
\text { fluoro, in } \mathrm{mg} / \mathrm{m}^{2}\end{array}$ & 2008-2009 & 2 & 0 & 19.8 & 20.7 & 09/24/08 & 19.0 & 20.7 & -- & -- & -- & -- & -- & -- \\
\hline Pheophytin a, periphyton, in $\mathrm{mg} / \mathrm{m}^{2}$ & 2003-2007 & 5 & 0 & 12.8 & 13.0 & $08 / 29 / 03$ & 0.8 & 13.0 & -- & -- & -- & -- & -- & -- \\
\hline Pheophytin a, periphyton, in $\mathrm{mg} / \mathrm{m}^{2}$ & 2008-2009 & 2 & 0 & 7.4 & 7.8 & 09/02/09 & 7.0 & 7.8 & -- & -- & -- & -- & -- & -- \\
\hline Cadmium, in micrograms per liter & 1995-2007 & 57 & 51 & 5 & 0.03 & ${ }^{4} 05 / 25 / 06$ & 5 & 5 & -- & 0.46 & 0 & 1.88 & 0 & $\mathrm{~L}$ \\
\hline Cadmium, in micrograms per liter & 2008-2009 & 12 & 4 & 0.01 & 0.04 & 05/05/09 & 5 & 0.03 & -- & 0.46 & 0 & 1.88 & 0 & $\mathrm{~L}$ \\
\hline Copper, in micrograms per liter & 1995-2007 & 57 & 17 & 0.63 & 2.9 & 07/12/06 & 5 & 1.1 & -- & 9.9 & 0 & 14.9 & 0 & $\mathrm{~L}$ \\
\hline Copper, in micrograms per liter & 2008-2009 & 12 & 5 & 0.55 & 4.1 & $08 / 18 / 08$ & 5 & 1.2 & -- & 9.9 & 0 & 14.9 & 0 & $\mathrm{~L}$ \\
\hline Lead, in micrograms per liter & 1995-2007 & 57 & 40 & 5 & 3.21 & 08/07/02 & 5 & 0.10 & -- & 2.84 & 1 & 72.9 & 0 & $\mathrm{~L}$ \\
\hline Lead, in micrograms per liter & 2008-2009 & 12 & 1 & 0.05 & 0.15 & 05/05/09 & 0.04 & 0.12 & -- & 2.84 & 0 & 72.9 & 0 & $\mathrm{~L}$ \\
\hline Manganese, in micrograms per liter & 1995-2007 & 89 & 0 & 15.0 & 38.0 & $03 / 16 / 95$ & 11.0 & 21.5 & -- & 1,712 & 0 & 3,099 & 0 & $\mathrm{~L}$ \\
\hline Manganese, in micrograms per liter & 2008-2009 & 12 & 0 & 16.9 & 39.2 & 03/10/08 & 11.6 & 23.9 & -- & 1,712 & 0 & 3,099 & 0 & $\mathrm{~L}$ \\
\hline Selenium, in micrograms per liter & $1995-2007$ & 41 & 21 & 5 & 0.50 & $04 / 25 / 02$ & 5 & 0.22 & -- & 4.6 & 0 & 18.4 & 0 & $\mathrm{~L}$ \\
\hline Selenium, in micrograms per liter & 2008-2009 & 12 & 0 & 0.12 & 0.26 & 05/05/09 & 0.10 & 0.17 & -- & 4.6 & 0 & 18.4 & 0 & $\mathrm{~L}$ \\
\hline
\end{tabular}


Table 21. Summary of measured constituents and properties for Gunnison River at CR 32 below Gunnison, Colorado, station 383103106594200. - Continued

[--, no data or not applicable; L, low; M, Medium; H, High; NTRU, Nephelometric Turbidity Ratio Unit; E. coli, Escherichia coli; mg/m², milligrams per square meter; USEPA, U.S. Environmental Protection Agency]

\begin{tabular}{|c|c|c|c|c|c|c|c|c|c|c|c|c|c|c|}
\hline $\begin{array}{l}\text { Property } \\
\text { or } \\
\text { constituent }\end{array}$ & $\begin{array}{l}\text { Period } \\
\text { (water } \\
\text { years) }\end{array}$ & $\begin{array}{l}\text { Number } \\
\text { of } \\
\text { samples }\end{array}$ & $\begin{array}{c}\text { Number } \\
\text { of } \\
\text { censored } \\
\text { values }\end{array}$ & Median' & Maximum & $\begin{array}{c}\text { Date } \\
\text { of } \\
\text { Maximum }\end{array}$ & $\begin{array}{c}\text { 15th } \\
\text { percentile }\end{array}$ & $\begin{array}{c}\text { 85th } \\
\text { percentile }^{1}\end{array}$ & $\begin{array}{c}\text { Geometric } \\
\text { Mean }^{2}\end{array}$ & $\begin{array}{c}\text { Standard } \\
\text { or } \\
\text { Chronic } \\
\text { standard }^{3}\end{array}$ & $\begin{array}{c}\text { Number of } \\
\text { exceedences } \\
\text { Standard or } \\
\text { Chronic }\end{array}$ & $\begin{array}{c}\text { Acute } \\
\text { standard }\end{array}$ & $\begin{array}{c}\begin{array}{c}\text { Number } \\
\text { of } \\
\text { exceedences } \\
\text { Acute }\end{array} \\
\end{array}$ & $\begin{array}{c}\text { Level } \\
\text { of } \\
\text { concern }\end{array}$ \\
\hline Silver, in micrograms per liter & $1995-2007$ & 49 & 49 & 5 & ${ }^{7}<1$ & -- & 5 & 5 & -- & 0.09 & 0 & 2.46 & 0 & $\mathrm{~L}$ \\
\hline Silver, in micrograms per liter & 2008-2009 & 12 & 12 & 5 & ${ }^{7}<0.1$ & -- & 5 & 5 & -- & 0.09 & 0 & 2.46 & 0 & $\mathrm{~L}$ \\
\hline Zinc, in micrograms per liter & $1995-2007$ & 57 & 20 & 1.2 & 15.8 & $04 / 27 / 01$ & 5 & 5.1 & -- & 137 & 0 & 158 & 0 & $\mathrm{~L}$ \\
\hline Zinc, in micrograms per liter & 2008-2009 & 12 & 2 & 1.8 & 6.7 & $02 / 10 / 09$ & 5 & 6.2 & -- & 137 & 0 & 158 & 0 & $\mathrm{~L}$ \\
\hline
\end{tabular}

${ }^{\prime}$ Censored values were replaced with 0 to compute median and 85 th percentiles (coliform censored values replaced with 1) see definitions section.

${ }^{2}$ Geometric mean listed for E. coli. Geometric mean computed for combined period of record (2001-2009).

${ }^{3}$ Colorado Department of Public Health and Environment, classification and numeric standards for Gunnison and Lower Dolores River Basins (2010a); and USEPA National Recommended Water Quality Criteria (2009). ${ }^{4}$ Multiple dates for maximum.

${ }^{5}$ Median values and percentiles were not tabled when they were calculated as zero.

${ }^{6}$ Use geometric mean for comparison to standard.

${ }^{7}$ All values below laboratory reporting level (LRL), current LRL used for current period maximum, highest LRL used for historic period. 
Table 22. Summary of seasonal Kendall trend tests for Gunnison River at CR 32 below Gunnison, Colorado, station 383103106594200 .

[E. coli, Escherichia coli]

\begin{tabular}{|c|c|c|c|c|}
\hline Property or constituent & $\begin{array}{c}\text { Period } \\
\text { (water years) }\end{array}$ & Kendall tau $(\tau)$ & p-Value & Trend direction \\
\hline Specific conductance, in microsiemens per centimeter & $1995-2009$ & 0.224 & 0.022 & Up \\
\hline $\mathrm{pH}$, in standard units & 1995-2009 & 0.111 & 0.264 & None \\
\hline Ammonia plus organic nitrogen, in milligrams per liter & 1999-2009 & 0.005 & 1.000 & None \\
\hline Nitrite plus nitrate nitrogen, in milligrams per liter & 1999-2009 & 0.041 & 0.755 & None \\
\hline Phosphorus (total), in milligrams per liter & 1999-2009 & -0.127 & 0.293 & None \\
\hline Orthophosphate, in milligrams per liter & 1999-2009 & -0.114 & 0.348 & None \\
\hline Calcium, in milligrams per liter & $1995-2009$ & 0.503 & $<0.001$ & $\mathrm{Up}$ \\
\hline Magnesium, in milligrams per liter & 1995-2009 & 0.381 & $<0.001$ & Up \\
\hline Copper, in micrograms per liter & 2003-2009 & -0.143 & 0.409 & None \\
\hline Iron, in micrograms per liter & $1995-2009$ & -0.169 & 0.325 & None \\
\hline Manganese, in micrograms per liter & 1995-2009 & 0.096 & 0.360 & None \\
\hline Zinc, in micrograms per liter & 2003-2009 & 0.202 & 0.228 & None \\
\hline Suspended sediment, in milligrams per liter & $1995-2009$ & -0.155 & 0.446 & None \\
\hline E. coli, in colonies per 100 milliliter & 2001-2009 & -0.117 & 0.426 & None \\
\hline
\end{tabular}




\section{Lake Fork Gunnison River below Deadmans Gulch near Lake City, Colorado}

Current Reason for Inclusion: To collect baseline data, characterize effects from mining, and facilitate remediation of the affected surface waters.

General Station Information:

Location:

Station Type:

Period of Record:

Latitude:

Longitude:
Private road bridge, 1/8 mi east of Highway 149, and 1.1 mi northeast of Lake City.

USGS water quality

Water quality: February 2007-September 2009

Drainage area: Not determined

HUC 14020002

USGS Data Summary: Water Years 2008-2009:

General Chemistry:

pH: Low concern

Dissolved oxygen: Low concern

Temperature: High concern

Nutrients:

Low concern

E. coli:

Low concern

Trace Elements/metals:

Low concern

Other constituents of concern: $\quad$ None for constituents tested.

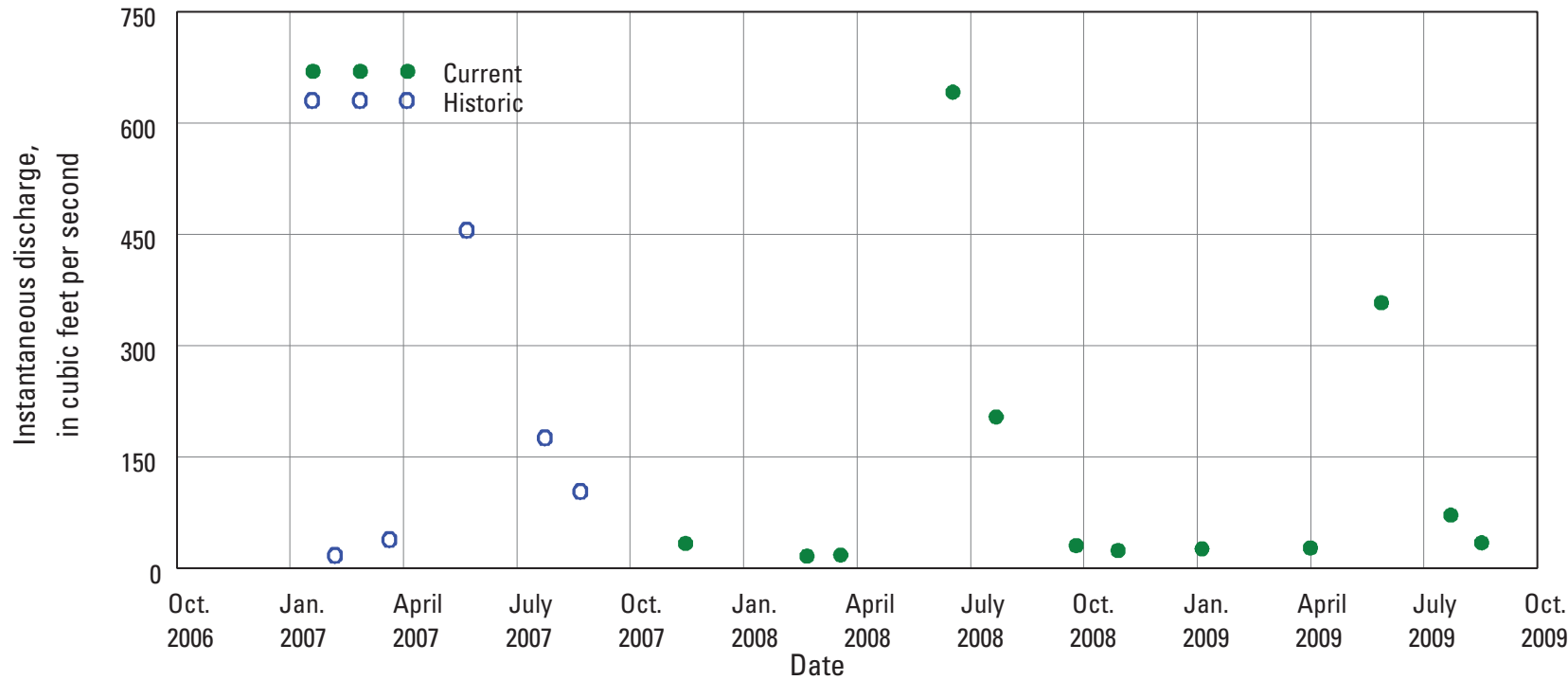

Figure 26. Time distribution and associated discharge of water-quality samples for Lake Fork Gunnison River below Deadmans Gulch, Colorado. 

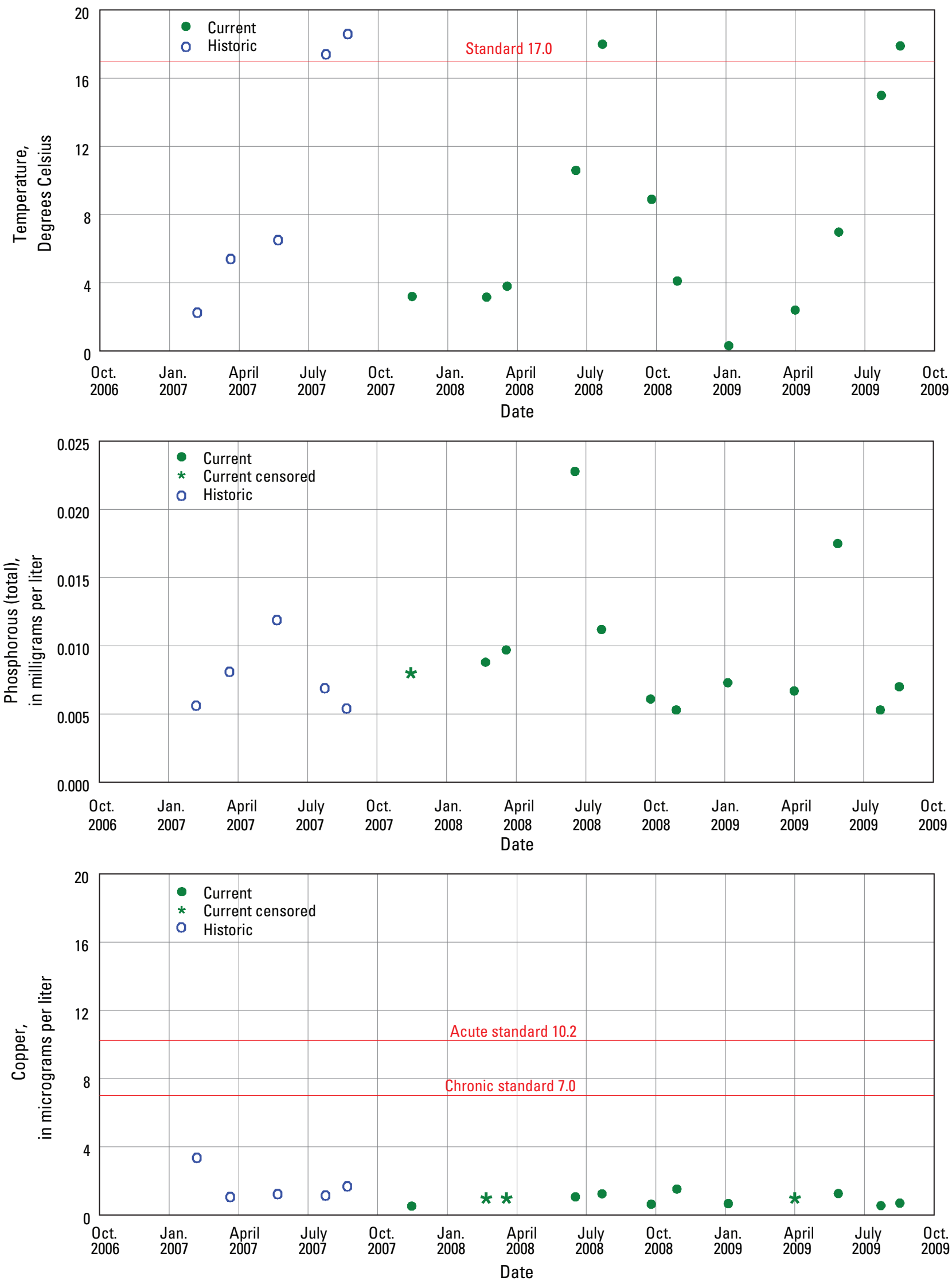

Figure 27. Time-series plots of selected water-quality constituents for Lake Fork Gunnison River below Deadmans Gulch, Colorado. 
Table 23. Summary of measured constituents and properties for Lake Fork Gunnison River below Deadmans Gulch, Colorado, station 09123490.

[--, no data or not applicable; L, low; M, Medium; H, High; NTRU, Nephelometric Turbidity Ratio Unit; E. coli, Escherichia coli; mg/m², milligrams per square meter; USEPA, U.S. Environmental Protection Agency]

\begin{tabular}{|c|c|c|c|c|c|c|c|c|c|c|c|c|c|c|}
\hline $\begin{array}{c}\text { Property } \\
\text { or } \\
\text { constituent }\end{array}$ & $\begin{array}{l}\text { Period } \\
\text { (water } \\
\text { years) }\end{array}$ & $\begin{array}{c}\text { Number } \\
\text { of } \\
\text { samples }\end{array}$ & $\begin{array}{c}\text { Number } \\
\text { of } \\
\text { censored } \\
\text { values }\end{array}$ & Median' & Maximum & $\begin{array}{c}\text { Date } \\
\text { of } \\
\text { Maximum }\end{array}$ & $\begin{array}{c}\text { 15th } \\
\text { percentile }\end{array}$ & $\begin{array}{c}\text { 85th } \\
\text { percentile }\end{array}$ & $\begin{array}{c}\text { Geometric } \\
\text { Mean }^{2}\end{array}$ & $\begin{array}{c}\text { Standard } \\
\text { or } \\
\text { Chronic } \\
\text { standard }^{3}\end{array}$ & $\begin{array}{l}\text { Number of } \\
\text { exceedences } \\
\text { Standard or } \\
\text { Chronic }\end{array}$ & $\begin{array}{l}\text { Acute } \\
\text { standard }\end{array}$ & $\begin{array}{c}\begin{array}{c}\text { Number } \\
\text { of }\end{array} \\
\text { exceedences } \\
\text { Acute }\end{array}$ & $\begin{array}{l}\text { Level } \\
\text { of } \\
\text { concern }\end{array}$ \\
\hline $\begin{array}{l}\text { Instantaneous discharge, in cubic } \\
\text { feet per second }\end{array}$ & 2007 & 5 & 0 & 104 & 456 & $05 / 22 / 07$ & 18 & 456 & -- & -- & -- & -- & -- & -- \\
\hline $\begin{array}{l}\text { Instantaneous discharge, in cubic } \\
\text { feet per second }\end{array}$ & 2008-2009 & 12 & 0 & 32 & 642 & $06 / 17 / 08$ & 18 & 372 & -- & -- & -- & -- & -- & -- \\
\hline Turbidity, in NTRU & 2007 & 5 & 4 & 0.0 & 4.6 & $05 / 22 / 07$ & 0.0 & 4.6 & -- & -- & -- & -- & -- & -- \\
\hline Turbidity, in NTRU & 2008-2009 & 12 & 8 & 0.0 & 6.3 & 05/28/09 & 0.0 & 4.9 & -- & -- & -- & -- & -- & -- \\
\hline $\begin{array}{l}\text { Dissolved oxygen, in milligrams } \\
\text { per liter }\end{array}$ & 2007 & 5 & 0 & 8.3 & 11.8 & $02 / 06 / 07$ & 6.2 & 11.8 & -- & 6.0 & 0 & -- & -- & $\mathrm{L}$ \\
\hline $\begin{array}{l}\text { Dissolved oxygen, in milligrams } \\
\text { per liter }\end{array}$ & 2008-2009 & 12 & 0 & 9.1 & 11.7 & $01 / 05 / 09$ & 7.4 & 10.3 & -- & 6.0 & 0 & -- & -- & $\mathrm{L}$ \\
\hline $\mathrm{pH}$, in standard units & 2007 & 5 & 0 & 8.0 & 8.2 & $08 / 22 / 07$ & 7.5 & 8.2 & -- & 6.5 & 0 & 9.0 & -- & $\mathrm{L}$ \\
\hline $\mathrm{pH}$, in standard units & 2008-2009 & 12 & 0 & 7.8 & 8.1 & 03/19/08 & 7.3 & 8.1 & -- & 6.5 & 0 & 9.0 & -- & $\mathrm{L}$ \\
\hline $\begin{array}{l}\text { Specific conductance, in microsie- } \\
\text { mens per centimeter }\end{array}$ & 2007 & 5 & 0 & 161 & 206 & $02 / 06 / 07$ & 140 & 206 & -- & -- & -- & -- & -- & -- \\
\hline $\begin{array}{l}\text { Specific conductance, in microsie- } \\
\text { mens per centimeter }\end{array}$ & 2008-2009 & 12 & 0 & 166 & 209 & 03/19/08 & 118 & 205 & -- & -- & -- & -- & -- & -- \\
\hline Temperature, degrees Celsius & 2007 & 5 & 0 & 6.5 & 18.6 & 08/22/07 & 2.2 & 18.6 & -- & 17.0 & 2 & -- & -- & $\mathrm{H}$ \\
\hline Temperature, degrees Celsius & 2008-2009 & 12 & 0 & 5.5 & 18.0 & $07 / 22 / 08$ & 2.3 & 17.9 & -- & 17.0 & 2 & -- & -- & $\mathrm{H}$ \\
\hline Calcium, in milligrams per liter & 2007 & 5 & 0 & 21.6 & 27.3 & $02 / 06 / 07$ & 18.9 & 27.3 & -- & -- & -- & -- & -- & -- \\
\hline Calcium, in milligrams per liter & $2008-2009$ & 12 & 0 & 22.3 & 28.9 & 03/19/08 & 14.7 & 27.1 & -- & -- & -- & -- & -- & -- \\
\hline Magnesium, in milligrams per liter & 2007 & 5 & 0 & 2.86 & 3.84 & $02 / 06 / 07$ & 2.51 & 3.84 & -- & -- & -- & -- & -- & -- \\
\hline Magnesium, in milligrams per liter & $2008-2009$ & 12 & 0 & 3.08 & 3.99 & $03 / 19 / 08$ & 1.89 & 3.74 & -- & -- & -- & -- & -- & -- \\
\hline $\begin{array}{l}\text { Ammonia plus organic nitrogen, in } \\
\text { milligrams per liter }\end{array}$ & 2007 & 5 & 2 & 0.06 & 0.11 & $02 / 06 / 07$ & 4 & 0.11 & -- & -- & -- & -- & -- & -- \\
\hline $\begin{array}{l}\text { Ammonia plus organic nitrogen, in } \\
\text { milligrams per liter }\end{array}$ & 2008-2009 & 12 & 7 & 4 & 0.22 & $05 / 28 / 09$ & ${ }^{4}$ & 0.15 & -- & -- & -- & -- & -- & -- \\
\hline $\begin{array}{l}\text { Ammonia nitrogen, in milligrams } \\
\text { per liter }\end{array}$ & 2007 & 5 & 4 & 4 & 0.017 & $03 / 21 / 07$ & 4 & 0.017 & -- & -- & -- & -- & -- & -- \\
\hline $\begin{array}{l}\text { Ammonia nitrogen, in milligrams } \\
\text { per liter }\end{array}$ & 2008-2009 & 12 & 11 & 4 & 0.011 & 04/01/09 & 4 & 0.001 & -- & -- & -- & -- & -- & -- \\
\hline $\begin{array}{l}\text { Nitrite plus nitrate nitrogen, in } \\
\text { milligrams per liter }\end{array}$ & 2007 & 5 & 2 & 0.019 & 0.057 & $05 / 22 / 07$ & 4 & 0.057 & -- & 10.0 & 0 & -- & -- & $\mathrm{L}$ \\
\hline $\begin{array}{l}\text { Nitrite plus nitrate nitrogen, in } \\
\text { milligrams per liter }\end{array}$ & 2008-2009 & 12 & 5 & 0.008 & 0.059 & 05/28/09 & 4 & 0.035 & -- & 10.0 & 0 & -- & -- & $\mathrm{L}$ \\
\hline $\begin{array}{l}\text { Nitrite nitrogen, in milligrams } \\
\text { per liter }\end{array}$ & 2007 & 5 & 1 & 0.001 & ${ }^{5}<0.002$ & -- & 4 & 4 & -- & 0.05 & 0 & -- & -- & L \\
\hline
\end{tabular}


Table 23. Summary of measured constituents and properties for Lake Fork Gunnison River below Deadmans Gulch, Colorado, station 09123490.—Continued

[--, no data or not applicable; L, low; M, Medium; H, High; NTRU, Nephelometric Turbidity Ratio Unit; E. coli, Escherichia coli; mg/m², milligrams per square meter; USEPA, U.S. Environmental Protection Agency]

\begin{tabular}{|c|c|c|c|c|c|c|c|c|c|c|c|c|c|c|}
\hline $\begin{array}{c}\text { Property } \\
\text { or } \\
\text { constituent }\end{array}$ & $\begin{array}{l}\text { Period } \\
\text { (water } \\
\text { years) }\end{array}$ & $\begin{array}{c}\text { Number } \\
\text { of } \\
\text { samples }\end{array}$ & $\begin{array}{l}\text { Number } \\
\text { of } \\
\text { censored } \\
\text { values }\end{array}$ & Median' & Maximum & $\begin{array}{c}\text { Date } \\
\text { of } \\
\text { Maximum }\end{array}$ & $\begin{array}{c}\text { 15th } \\
\text { percentile }\end{array}$ & $\begin{array}{c}\text { 85th } \\
\text { percentile }\end{array}$ & $\begin{array}{c}\text { Geometric } \\
\text { Mean }^{2}\end{array}$ & $\begin{array}{l}\text { Standard } \\
\text { or } \\
\text { Chronic } \\
\text { standard }^{3}\end{array}$ & $\begin{array}{l}\text { Number of } \\
\text { exceedences } \\
\text { Standard or } \\
\text { Chronic }\end{array}$ & $\begin{array}{l}\text { Acute } \\
\text { standard }\end{array}$ & $\begin{array}{c}\begin{array}{c}\text { Number } \\
\text { of }\end{array} \\
\text { exceedences } \\
\text { Acute }\end{array}$ & $\begin{array}{c}\text { Level } \\
\text { of } \\
\text { concern }\end{array}$ \\
\hline $\begin{array}{l}\text { Nitrite nitrogen, in milligrams } \\
\text { per liter }\end{array}$ & 2008-2009 & 12 & 11 & 4 & ${ }^{s}<0.002$ & -- & 4 & 4 & -- & 0.05 & 0 & -- & -- & $\mathrm{L}$ \\
\hline $\begin{array}{l}\text { Orthophosphate, in milligrams } \\
\text { per liter }\end{array}$ & 2007 & 5 & 2 & 0.003 & ${ }^{s}<0.006$ & -- & 4 & 4 & -- & -- & -- & -- & -- & -- \\
\hline $\begin{array}{l}\text { Orthophosphate, in milligrams } \\
\text { per liter }\end{array}$ & 2008-2009 & 12 & 3 & 0.004 & ${ }^{5}<0.008$ & -- & 4 & 4 & -- & -- & -- & -- & -- & -- \\
\hline $\begin{array}{l}\text { Phosphorus (total), in milligrams } \\
\text { per liter }\end{array}$ & 2007 & 5 & 0 & 0.007 & 0.012 & $05 / 22 / 07$ & 0.005 & 0.012 & -- & -- & -- & -- & -- & -- \\
\hline $\begin{array}{l}\text { Phosphorus (total), in milligrams } \\
\text { per liter }\end{array}$ & 2008-2009 & 12 & 1 & 0.007 & 0.023 & 06/17/08 & 0.005 & 0.018 & -- & -- & -- & -- & -- & -- \\
\hline $\begin{array}{l}\text { E. coli, in colonies per } 100 \text { mil- } \\
\text { liliter }\end{array}$ & 2007 & 5 & 2 & 1 & 4 & $07 / 24 / 07$ & 1 & 6 & 1.7 & 126 & 0 & -- & -- & $\mathrm{L}$ \\
\hline $\begin{array}{l}\text { E. coli, in colonies per } 100 \text { mil- } \\
\quad \text { liliter }\end{array}$ & 2008-2009 & 10 & 5 & 1 & 8 & $07 / 22 / 08$ & 1 & 6 & -- & 126 & 0 & -- & -- & $\mathrm{L}$ \\
\hline $\begin{array}{l}\text { Biomass periphyton, ashfree dry- } \\
\text { mass, in grams per square meter }\end{array}$ & 2007 & 1 & 1 & -- & (5) & $08 / 22 / 07$ & -- & -- & -- & -- & -- & -- & -- & -- \\
\hline $\begin{array}{l}\text { Biomass periphyton, ashfree dry- } \\
\text { mass, in grams per square meter }\end{array}$ & 2008-2009 & 2 & 1 & 2.0 & 3.9 & $09 / 25 / 08$ & 4 & 3.9 & -- & -- & -- & -- & -- & -- \\
\hline $\begin{array}{l}\text { Periphyton, biomass, ash weight, } \\
\text { in grams per square meter }\end{array}$ & 2007 & 1 & 0 & -- & 411 & 08/22/07 & -- & -- & -- & -- & -- & -- & -- & -- \\
\hline $\begin{array}{l}\text { Periphyton, biomass, ash weight, } \\
\text { in grams per square meter }\end{array}$ & 2008-2009 & 2 & 0 & 264 & 426 & 08/17/09 & 103 & 426 & -- & -- & -- & -- & -- & -- \\
\hline $\begin{array}{l}\text { Periphyton, biomass, dry weight, } \\
\text { in grams per square meter }\end{array}$ & 2007 & 1 & 0 & -- & 416 & 08/22/07 & -- & -- & -- & -- & -- & -- & -- & -- \\
\hline $\begin{array}{l}\text { Periphyton, biomass, dry weight, } \\
\text { in grams per square meter }\end{array}$ & 2008-2009 & 2 & 0 & 270 & 434 & 08/17/09 & 106 & 434 & -- & -- & -- & -- & -- & -- \\
\hline $\begin{array}{l}\text { Chlorophyll a, periphyton, chromo- } \\
\text { fluoro, in } \mathrm{mg} / \mathrm{m}^{2}\end{array}$ & 2007 & 1 & 0 & -- & 6.47 & $08 / 22 / 07$ & -- & -- & -- & -- & -- & -- & -- & -- \\
\hline $\begin{array}{l}\text { Chlorophyll a, periphyton, chromo- } \\
\text { fluoro, in } \mathrm{mg} / \mathrm{m}^{2}\end{array}$ & 2008-2009 & 2 & 0 & 9.70 & 12.2 & 08/17/09 & 7.15 & 12.2 & -- & -- & -- & -- & -- & -- \\
\hline Pheophytin a, periphyton, in $\mathrm{mg} / \mathrm{m}^{2}$ & 2007 & 1 & 0 & -- & 2.3 & $08 / 22 / 07$ & -- & -- & -- & -- & -- & -- & -- & -- \\
\hline Pheophytin a, periphyton, in $\mathrm{mg} / \mathrm{m}^{2}$ & 2008-2009 & 2 & 0 & 4.1 & 4.9 & 08/17/09 & 3.2 & 4.9 & -- & -- & -- & -- & -- & -- \\
\hline Aluminum, in micrograms per liter & 2007 & 5 & 0 & 30.3 & 75.4 & $08 / 22 / 07$ & 18.2 & 75.4 & -- & -- & -- & -- & -- & -- \\
\hline Aluminum, in micrograms per liter & $2008-2009$ & 12 & 0 & 37.7 & 88.2 & 07/23/09 & 19.9 & 70.9 & -- & -- & -- & -- & -- & -- \\
\hline Cadmium, in micrograms per liter & 2007 & 5 & 2 & 0.02 & 0.03 & $05 / 22 / 07$ & 4 & 0.03 & -- & 0.34 & 0 & 1.32 & 0 & $\mathrm{~L}$ \\
\hline Cadmium, in micrograms per liter & 2008-2009 & 12 & 4 & 0.01 & 0.03 & $07 / 23 / 09$ & 4 & 0.03 & -- & 0.34 & 0 & 1.32 & 0 & $\mathrm{~L}$ \\
\hline Copper, in micrograms per liter & 2007 & 5 & 0 & 1.2 & 3.4 & $02 / 06 / 07$ & 1.1 & 3.4 & -- & 7.0 & 0 & 10.2 & 0 & $\mathrm{~L}$ \\
\hline
\end{tabular}


Table 23. Summary of measured constituents and properties for Lake Fork Gunnison River below Deadmans Gulch, Colorado, station 09123490.—Continued

[--, no data or not applicable; L, low; M, Medium; H, High; NTRU, Nephelometric Turbidity Ratio Unit; E. coli, Escherichia coli; mg/m², milligrams per square meter; USEPA, U.S. Environmental Protection Agency]

\begin{tabular}{|c|c|c|c|c|c|c|c|c|c|c|c|c|c|c|}
\hline $\begin{array}{l}\text { Property } \\
\text { or } \\
\text { constituent }\end{array}$ & $\begin{array}{l}\text { Period } \\
\text { (water } \\
\text { years) }\end{array}$ & $\begin{array}{l}\text { Number } \\
\text { of } \\
\text { samples }\end{array}$ & $\begin{array}{c}\text { Number } \\
\text { of } \\
\text { censored } \\
\text { values }\end{array}$ & Median' & Maximum & $\begin{array}{c}\text { Date } \\
\text { of } \\
\text { Maximum }\end{array}$ & $\begin{array}{c}\text { 15th } \\
\text { percentile }\end{array}$ & $\begin{array}{c}\text { 85th } \\
\text { percentile' }\end{array}$ & $\begin{array}{c}\text { Geometric } \\
\text { Mean }^{2}\end{array}$ & $\begin{array}{c}\text { Standard } \\
\text { or } \\
\text { Chronic } \\
\text { standard }^{3}\end{array}$ & $\begin{array}{l}\text { Number of } \\
\text { exceedences } \\
\text { Standard or } \\
\text { Chronic }\end{array}$ & $\begin{array}{l}\text { Acute } \\
\text { standard }\end{array}$ & $\begin{array}{c}\begin{array}{c}\text { Number } \\
\text { of } \\
\text { exceedences } \\
\text { Acute }\end{array} \\
\end{array}$ & $\begin{array}{c}\text { Level } \\
\text { of } \\
\text { concern }\end{array}$ \\
\hline Copper, in micrograms per liter & $2008-2009$ & 12 & 3 & 0.65 & 1.5 & $10 / 29 / 08$ & 4 & 1.3 & -- & 7.0 & 0 & 10.2 & 0 & $\mathrm{~L}$ \\
\hline Iron, in micrograms per liter & 2007 & 5 & 1 & 19 & 31 & $07 / 24 / 07$ & 4 & 31 & -- & 300 & 0 & -- & -- & $\mathrm{L}$ \\
\hline Iron, in micrograms per liter & 2008-2009 & 12 & 0 & 21 & 37 & 05/28/09 & 13 & 37 & -- & 300 & 0 & -- & -- & $\mathrm{L}$ \\
\hline Lead, in micrograms per liter & 2007 & 5 & 1 & 0.06 & 0.13 & $02 / 06 / 07$ & 4 & 0.13 & -- & 1.83 & 0 & 47.1 & 0 & $\mathrm{~L}$ \\
\hline Lead, in micrograms per liter & 2008-2009 & 12 & 9 & 4 & 0.05 & $05 / 28 / 09$ & 4 & 0.05 & -- & 1.83 & 0 & 47.1 & 0 & $\mathrm{~L}$ \\
\hline Manganese, in micrograms per liter & 2007 & 5 & 0 & 68.5 & 151 & $05 / 22 / 07$ & 32.4 & 151 & -- & 1,498 & 0 & 2,712 & 0 & $\mathrm{~L}$ \\
\hline Manganese, in micrograms per liter & $2008-2009$ & 12 & 0 & 52.2 & 101 & 06/17/08 & 39.2 & 74.4 & -- & 1,498 & 0 & 2,712 & 0 & $\mathrm{~L}$ \\
\hline Silver, in micrograms per liter & 2007 & 5 & 5 & 4 & ${ }^{5}<0.1$ & -- & 4 & 4 & -- & 0.05 & 0 & 1.23 & 0 & $\mathrm{~L}$ \\
\hline Silver, in micrograms per liter & $2008-2009$ & 12 & 11 & 4 & 0.03 & 07/23/09 & 4 & 4 & -- & 0.05 & 0 & 1.23 & 0 & $\mathrm{~L}$ \\
\hline Zinc, in micrograms per liter & 2007 & 5 & 0 & 3.4 & 6.8 & $02 / 06 / 07$ & 2.0 & 6.8 & -- & 55.0 & 0 & 112 & 0 & $\mathrm{~L}$ \\
\hline Zinc, in micrograms per liter & 2008-2009 & 12 & 0 & 2.0 & 5.5 & 07/22/08 & 1.6 & 3.1 & -- & 55.0 & 0 & 112 & 0 & $\mathrm{~L}$ \\
\hline $\begin{array}{l}\text { Suspended sediment, in milligrams } \\
\text { per liter }\end{array}$ & 2007 & 5 & 0 & 2.0 & 4.0 & $05 / 22 / 07$ & 1.0 & 4.0 & -- & -- & -- & -- & -- & -- \\
\hline $\begin{array}{l}\text { Suspended sediment, in milligrams } \\
\text { per liter }\end{array}$ & 2008-2009 & 12 & 1 & 1.0 & 11.0 & 06/17/08 & 0.9 & 6.2 & -- & -- & -- & -- & -- & -- \\
\hline
\end{tabular}
per liter

Censored values were replaced with 0 to compute median and 85 th percentiles (coliform censored values replaced with 1 ) see definitions section.

${ }^{2}$ Geometric mean listed for $E$. coli. Geometric mean computed for combined period of record (2007-2009).

${ }^{3}$ Colorado Department of Public Health and Environment, classification and numeric standards for Gunnison and Lower Dolores River Basins (2010a); and USEPA National Recommended Water Quality Criteria (2009). ${ }^{4}$ Median values and percentiles were not tabled when they were calculated as zero.

${ }^{5}$ All values below laboratory reporting level (LRL), current LRL used for current period maximum, highest LRL used for historic period.

${ }^{6}$ Use geometric mean for comparison to standard. 


\section{Cebolla Creek at Powderhorn, Colorado}

Current Reason for Inclusion: To collect baseline data, characterize impacts from mining, and facilitate remediation of the affected surface waters. This station is part of the rotational group of sites.

\section{General Station Information:}

Location:

Station Type:

Period of Record:

Latitude: 381633

Longitude: 1070547
Private road bridge, $1.4 \mathrm{mi}$ south of State Highway 149, and $0.1 \mathrm{mi}$ southwest of Powderhorn.

USGS water quality

Water quality: June 1999-September 2009

Data collected by NPS and reviewed by USGS June 1999-Oct 2005

Data collected by USGS water year 2007-2009

Drainage area: Not determined

HUC 14020002

USGS Data Summary: Water Years 2008-2009:

General Chemistry:

Nutrients:

E. coli:

Trace Elements/metals:

Other constituents of concern:
pH: Low concern

Dissolved oxygen: Low concern

Temperature: Low concern

Low concern

Low concern

Low concern

None for constituents tested.

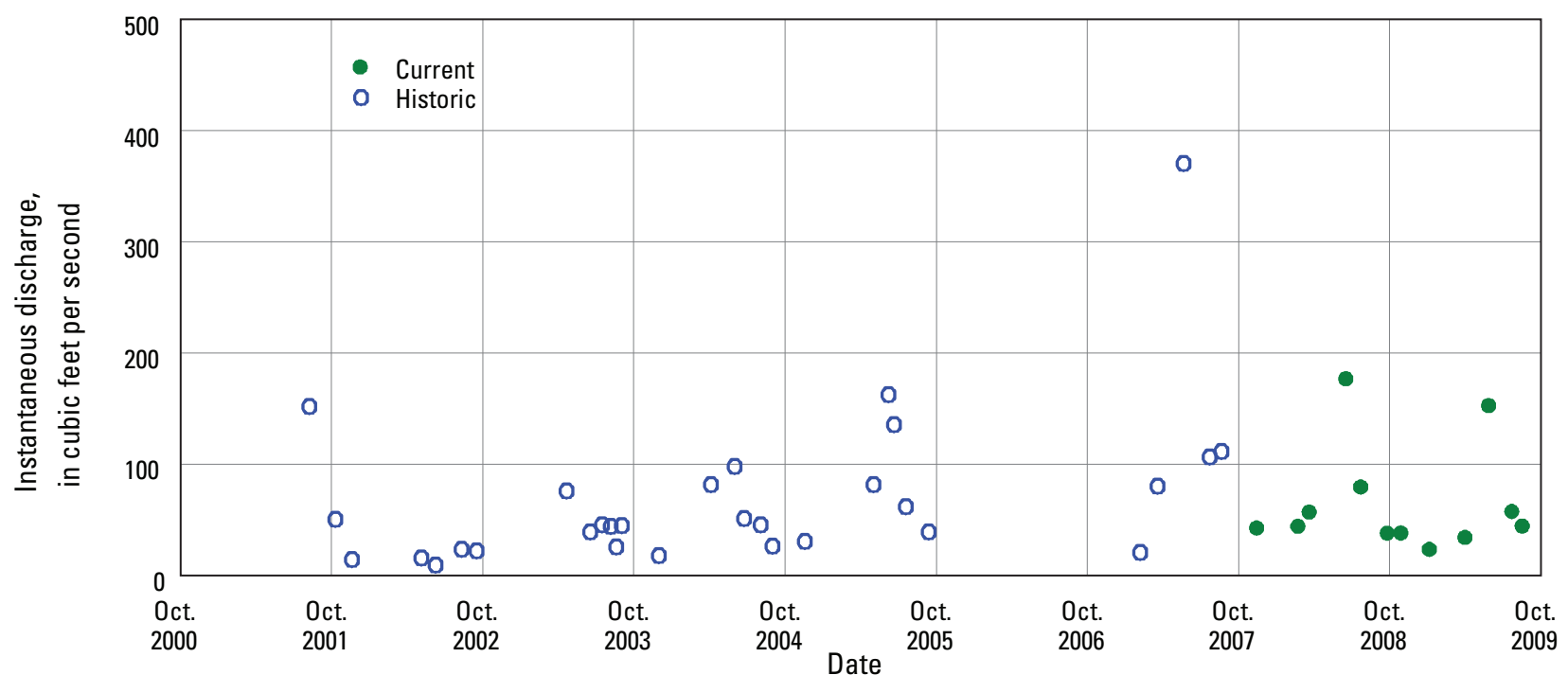

Figure 28. Time distribution and associated discharge of water-quality samples for Cebolla Creek at Powderhorn, Colorado. 

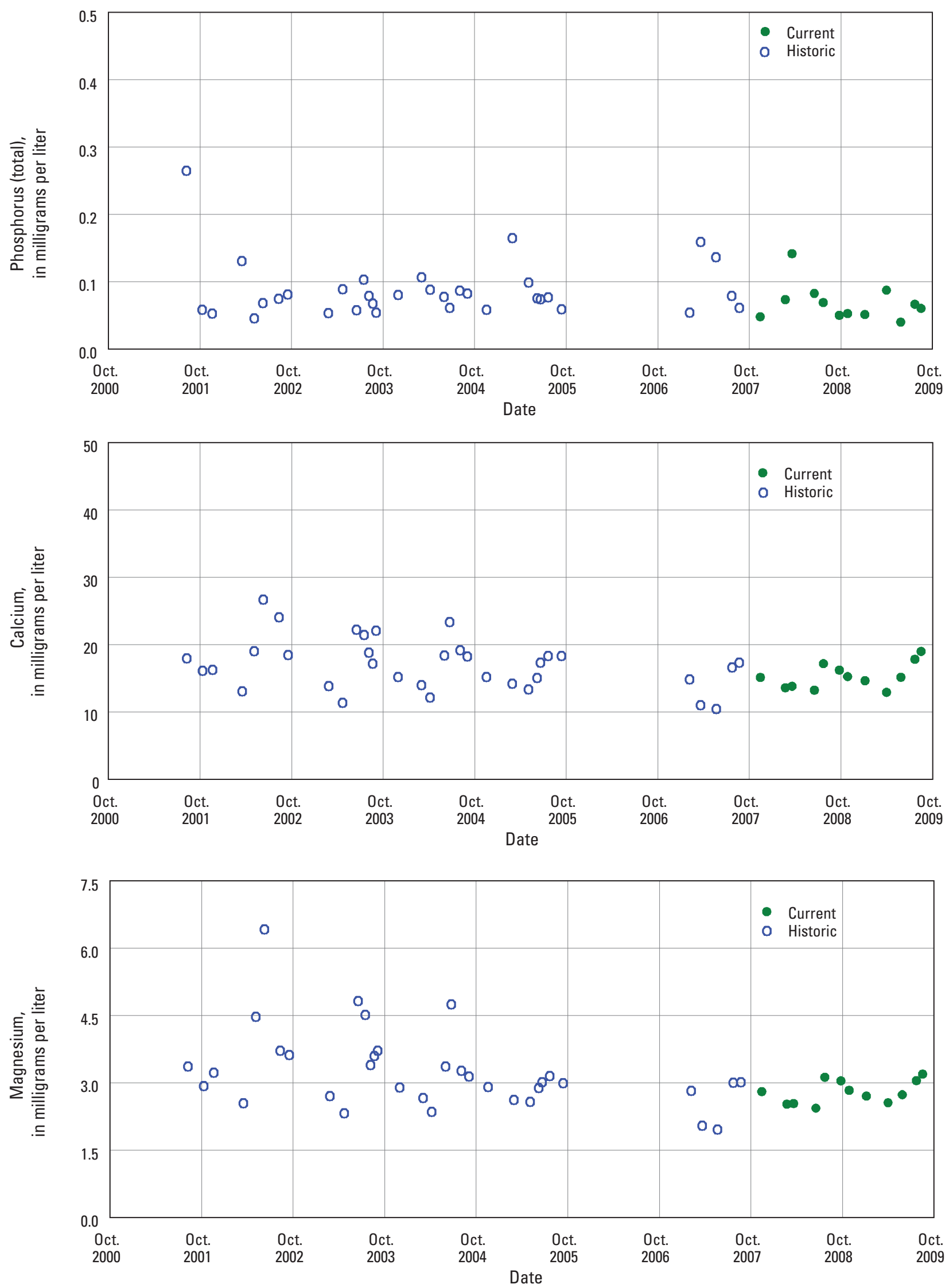

Figure 29. Time-series plots of selected water-quality constituents for Cebolla Creek at Powderhorn, Colorado. 
Table 24. Summary of measured constituents and properties for Cebolla Creek at Powderhorn, Colorado, station 381633107054700.

[--, no data or not applicable; L, low; M, Medium; H, High; NTRU, Nephelometric Turbidity Ratio Unit; E. coli, Escherichia coli; mg/m² milligrams per square meter; USEPA, U.S. Environmental Protection Agency]

\begin{tabular}{|c|c|c|c|c|c|c|c|c|c|c|c|c|c|c|}
\hline $\begin{array}{c}\text { Property } \\
\text { or } \\
\text { constituent }\end{array}$ & $\begin{array}{l}\text { Period } \\
\text { (water } \\
\text { years) }\end{array}$ & $\begin{array}{c}\text { Number } \\
\text { of } \\
\text { samples }\end{array}$ & $\begin{array}{l}\text { Number } \\
\text { of } \\
\text { censored } \\
\text { values }\end{array}$ & Median' $^{1}$ & Maximum & $\begin{array}{c}\text { Date } \\
\text { of } \\
\text { Maximum }\end{array}$ & $\begin{array}{c}\text { 15th } \\
\text { percentile }\end{array}$ & $\begin{array}{c}\text { 85th } \\
\text { percentile }\end{array}$ & $\begin{array}{l}\text { Geometric } \\
\text { Mean }^{2}\end{array}$ & $\begin{array}{l}\text { Standard } \\
\text { or } \\
\text { Chronic } \\
\text { standard }^{3}\end{array}$ & $\begin{array}{l}\text { Number of } \\
\text { exceedences } \\
\text { Standard or } \\
\text { Chronic }\end{array}$ & $\begin{array}{c}\text { Acute } \\
\text { standard }\end{array}$ & $\begin{array}{c}\begin{array}{c}\text { Number } \\
\text { of }\end{array} \\
\text { exceedences } \\
\text { Acute }\end{array}$ & $\begin{array}{c}\text { Level } \\
\text { of } \\
\text { concern }\end{array}$ \\
\hline $\begin{array}{l}\text { Instantaneous discharge, in cubic feet } \\
\text { per second }\end{array}$ & 1999-2007 & 34 & 0 & 48 & 468 & 06/01/99 & 21 & 148 & -- & -- & -- & -- & -- & -- \\
\hline $\begin{array}{l}\text { Instantaneous discharge, in cubic feet } \\
\text { per second }\end{array}$ & 2008-2009 & 12 & 0 & 44 & 177 & 06/17/08 & 34 & 154 & -- & -- & -- & -- & -- & -- \\
\hline Turbidity, in NTRU & 2008-2009 & 12 & 1 & 2.0 & 5.3 & $03 / 20 / 08$ & 1.2 & 4.1 & -- & -- & -- & -- & -- & -- \\
\hline $\begin{array}{l}\text { Dissolved oxygen, in milligrams } \\
\text { per liter }\end{array}$ & 1999-2007 & 38 & 0 & 8.7 & 13.3 & $02 / 06 / 07$ & 7.4 & 10.6 & -- & 6.0 & 0 & -- & -- & $\mathrm{L}$ \\
\hline $\begin{array}{l}\text { Dissolved oxygen, in milligrams } \\
\text { per liter }\end{array}$ & 2008-2009 & 12 & 0 & 8.9 & 11.6 & 03/20/08 & 7.6 & 11.4 & -- & 6.0 & 0 & -- & -- & $\mathrm{L}$ \\
\hline $\mathrm{pH}$, in standard units & 1999-2007 & 36 & 0 & 7.8 & 8.6 & $09 / 02 / 04$ & 7.5 & 8.0 & -- & 6.5 & 0 & 9.0 & -- & $\mathrm{L}$ \\
\hline $\mathrm{pH}$, in standard units & 2008-2009 & 12 & 0 & 7.7 & 8.1 & 09/25/08 & 7.0 & 8.1 & -- & 6.5 & 1 & 9.0 & -- & $\mathrm{L}$ \\
\hline $\begin{array}{l}\text { Specific conductance, in microsie- } \\
\text { mens per centimeter }\end{array}$ & 1999-2007 & 38 & 0 & 134 & 232 & $06 / 10 / 02$ & 109 & 167 & -- & -- & -- & -- & -- & -- \\
\hline $\begin{array}{l}\text { Specific conductance, in microsie- } \\
\text { mens per centimeter }\end{array}$ & 2008-2009 & 12 & 0 & 127 & 153 & 08/17/09 & 111 & 144 & -- & -- & -- & -- & -- & -- \\
\hline Temperature, degrees Celsius & 1999-2007 & 38 & 0 & 7.8 & 21.9 & $07 / 17 / 03$ & 0.0 & 14.7 & -- & 17.0 & 3 & -- & -- & $\mathrm{L}$ \\
\hline Temperature, degrees Celsius & 2008-2009 & 12 & 0 & 8.9 & 17.1 & 07/23/09 & 0.0 & 16.6 & -- & 17.0 & 1 & -- & -- & $\mathrm{L}$ \\
\hline Calcium, in milligrams per liter & $2001-2007$ & 34 & 0 & 17.3 & 26.7 & $06 / 10 / 02$ & 13.1 & 22.0 & -- & -- & -- & -- & -- & -- \\
\hline Calcium, in milligrams per liter & 2008-2009 & 12 & 0 & 15.2 & 19.0 & 08/17/09 & 13.2 & 17.9 & -- & -- & -- & -- & -- & -- \\
\hline Magnesium, in milligrams per liter & 2001-2007 & 34 & 0 & 3.02 & 6.43 & $06 / 10 / 02$ & 2.56 & 4.28 & -- & -- & -- & -- & -- & -- \\
\hline Magnesium, in milligrams per liter & $2008-2009$ & 12 & 0 & 2.77 & 3.20 & 08/17/09 & 2.53 & 3.13 & -- & -- & -- & -- & -- & -- \\
\hline Chloride, in milligrams per liter & 2007 & 5 & 0 & 0.71 & 1.10 & $03 / 21 / 07$ & 0.64 & 1.10 & -- & 250 & 0 & -- & -- & $\mathrm{L}$ \\
\hline Chloride, in milligrams per liter & 2008-2009 & 12 & 0 & 0.85 & 1.20 & 04/01/09 & 0.77 & 1.04 & -- & 250 & 0 & -- & -- & $\mathrm{L}$ \\
\hline $\begin{array}{l}\text { Ammonia plus organic nitrogen, in } \\
\text { milligrams per liter }\end{array}$ & 1999-2004 & 26 & 0 & 0.20 & 0.42 & 06/01/99 & 0.12 & 0.36 & -- & -- & -- & -- & -- & -- \\
\hline $\begin{array}{l}\text { Ammonia plus organic nitrogen, in } \\
\text { milligrams per liter }\end{array}$ & $2008-2009$ & 12 & 0 & 0.16 & 0.33 & 05/28/09 & 0.07 & 0.28 & -- & -- & -- & -- & -- & -- \\
\hline $\begin{array}{l}\text { Ammonia nitrogen, in milligrams } \\
\text { per liter }\end{array}$ & 1999-2007 & 38 & 29 & 4 & 0.014 & $03 / 21 / 07$ & 4 & 0.007 & -- & 3.17 & 0 & 8.79 & 0 & $\mathrm{~L}$ \\
\hline $\begin{array}{l}\text { Ammonia nitrogen, in milligrams } \\
\text { per liter }\end{array}$ & 2008-2009 & 12 & 11 & 4 & 0.012 & 07/23/09 & 4 & 0.001 & -- & 3.69 & 0 & 12.6 & 0 & $\mathrm{~L}$ \\
\hline $\begin{array}{l}\text { Nitrite plus nitrate nitrogen, in mil- } \\
\text { ligrams per liter }\end{array}$ & 1999-2007 & 38 & 22 & 4 & 0.145 & $02 / 06 / 07$ & 4 & 0.034 & -- & 10.0 & 0 & -- & -- & $\mathrm{L}$ \\
\hline $\begin{array}{l}\text { Nitrite plus nitrate nitrogen, in mil- } \\
\text { ligrams per liter }\end{array}$ & 2008-2009 & 12 & 7 & ${ }^{4}$ & 0.153 & $02 / 22 / 08$ & 4 & 0.132 & -- & 10.0 & 0 & -- & -- & $\mathrm{L}$ \\
\hline $\begin{array}{l}\text { Nitrite nitrogen, in milligrams per } \\
\text { liter }\end{array}$ & 1999-2007 & 38 & 24 & 4 & 0.002 & 03/18/02 & 4 & 0.001 & -- & 0.05 & 0 & -- & -- & $\mathrm{L}$ \\
\hline
\end{tabular}


Table 24. Summary of measured constituents and properties for Cebolla Creek at Powderhorn, Colorado, station 381633107054700.-Continued

[--, no data or not applicable; L, low; M, Medium; H, High; NTRU, Nephelometric Turbidity Ratio Unit; E. coli, Escherichia coli; mg/m² , milligrams per square meter; USEPA, U.S. Environmental Protection Agency]

\begin{tabular}{|c|c|c|c|c|c|c|c|c|c|c|c|c|c|c|}
\hline $\begin{array}{l}\text { Property } \\
\text { or } \\
\text { constituent }\end{array}$ & $\begin{array}{l}\text { Period } \\
\text { (water } \\
\text { years) }\end{array}$ & $\begin{array}{c}\text { Number } \\
\text { of } \\
\text { samples }\end{array}$ & $\begin{array}{c}\begin{array}{c}\text { Number } \\
\text { of }\end{array} \\
\text { censored } \\
\text { values }\end{array}$ & Median $^{1}$ & Maximum & $\begin{array}{c}\text { Date } \\
\text { of } \\
\text { Maximum }\end{array}$ & $\begin{array}{c}\text { 15th } \\
\text { percentile }\end{array}$ & $\begin{array}{c}\text { 85th } \\
\text { percentile }\end{array}$ & $\begin{array}{c}\text { Geometric } \\
\text { Mean }^{2}\end{array}$ & $\begin{array}{c}\text { Standard } \\
\text { or } \\
\text { Chronic } \\
\text { standard }^{3}\end{array}$ & $\begin{array}{c}\text { Number of } \\
\text { exceedences } \\
\text { Standard or } \\
\text { Chronic }\end{array}$ & $\begin{array}{l}\text { Acute } \\
\text { standard }\end{array}$ & $\begin{array}{c}\begin{array}{c}\text { Number } \\
\text { of }\end{array} \\
\text { exceedences } \\
\text { Acute }\end{array}$ & $\begin{array}{c}\text { Level } \\
\text { of } \\
\text { concern }\end{array}$ \\
\hline $\begin{array}{l}\text { Nitrite nitrogen, in milligrams per } \\
\text { liter }\end{array}$ & 2008-2009 & 12 & 8 & 4 & ${ }^{5}<0.002$ & -- & 4 & 4 & -- & 0.05 & 0 & -- & -- & $\mathrm{L}$ \\
\hline $\begin{array}{l}\text { Orthophosphate, in milligrams per } \\
\text { liter }\end{array}$ & 1999-2004 & 26 & 0 & 0.036 & 0.054 & $08 / 12 / 02$ & 0.031 & 0.047 & -- & -- & -- & -- & -- & -- \\
\hline $\begin{array}{l}\text { Orthophosphate, in milligrams per } \\
\text { liter }\end{array}$ & 2008-2009 & 12 & 0 & 0.041 & 0.052 & $07 / 23 / 08$ & 0.032 & 0.046 & -- & -- & -- & -- & -- & -- \\
\hline $\begin{array}{l}\text { Phosphorus (total), in milligrams } \\
\text { per liter }\end{array}$ & 1999-2007 & 38 & 0 & 0.077 & 0.265 & 08/09/01 & 0.057 & 0.132 & -- & -- & -- & -- & -- & -- \\
\hline $\begin{array}{l}\text { Phosphorus (total), in milligrams } \\
\text { per liter }\end{array}$ & 2008-2009 & 12 & 0 & 0.064 & 0.142 & 03/20/08 & 0.048 & 0.090 & -- & -- & -- & -- & -- & -- \\
\hline E. coli, in colonies per 100 milliliter & 2007 & 5 & 0 & 18 & 100 & $07 / 24 / 07$ & 2 & 6 & 7.6 & 126 & 0 & -- & -- & M \\
\hline E. coli, in colonies per 100 milliliter & 2008-2009 & 10 & 1 & 9 & 27 & $07 / 23 / 08$ & 1 & 6 & -- & 126 & 0 & -- & -- & $\mathrm{L}$ \\
\hline $\begin{array}{l}\text { Biomass periphyton, ashfree drymass, } \\
\text { in grams per square meter }\end{array}$ & 2008 & 1 & 0 & -- & 4.10 & $09 / 25 / 08$ & -- & -- & -- & -- & -- & -- & -- & -- \\
\hline $\begin{array}{l}\text { Periphyton, biomass, ash weight, in } \\
\text { grams per square meter }\end{array}$ & 2008 & 1 & 0 & -- & 146 & $09 / 25 / 08$ & -- & -- & -- & -- & -- & -- & -- & -- \\
\hline $\begin{array}{l}\text { Periphyton, biomass, dry weight, in } \\
\text { grams per square meter }\end{array}$ & 2008 & 1 & 0 & -- & 150 & $09 / 25 / 08$ & -- & -- & -- & -- & -- & -- & -- & -- \\
\hline $\begin{array}{l}\text { Chlorophyll a, periphyton, chromo- } \\
\quad \text { fluoro, in } \mathrm{mg} / \mathrm{m}^{2}\end{array}$ & 2008 & 1 & 0 & -- & 4.76 & $09 / 25 / 08$ & -- & -- & -- & -- & -- & -- & -- & -- \\
\hline Pheophytin a, periphyton, in $\mathrm{mg} / \mathrm{m}^{2}$ & 2008 & 1 & 0 & -- & 1.0 & $09 / 25 / 08$ & -- & -- & -- & -- & -- & -- & -- & -- \\
\hline Aluminum, in micrograms per liter & 2008-2009 & 12 & 0 & 20.5 & 38.6 & $05 / 28 / 09$ & 14.4 & 30.6 & -- & -- & -- & -- & -- & -- \\
\hline Arsenic, in micrograms per liter & 2007 & 5 & 0 & 0.52 & 0.62 & $07 / 24 / 07$ & 0.46 & 0.62 & -- & -- & -- & -- & -- & -- \\
\hline Arsenic, in micrograms per liter & 2008-2009 & 12 & 0 & 0.61 & 0.73 & $08 / 17 / 09$ & 0.48 & 0.73 & -- & -- & -- & -- & -- & -- \\
\hline $\begin{array}{l}\text { Arsenic (total), in micrograms per } \\
\text { liter }\end{array}$ & 2007 & 5 & 0 & 0.75 & 0.90 & $05 / 22 / 07$ & 0.71 & 0.90 & -- & -- & -- & 340 & 0 & -- \\
\hline $\begin{array}{l}\text { Arsenic (total), in micrograms per } \\
\text { liter }\end{array}$ & 2008-2009 & 12 & 0 & 0.70 & 1.1 & 08/17/09 & 0.61 & 0.92 & -- & -- & -- & 340 & 0 & -- \\
\hline Barium, in micrograms per liter & 2007 & 5 & 0 & 18 & 21 & $07 / 24 / 07$ & 15 & 21 & -- & -- & -- & -- & -- & -- \\
\hline Barium, in micrograms per liter & 2008-2009 & 12 & 0 & 21 & 25 & 07/23/08 & 17 & 23 & -- & -- & -- & -- & -- & -- \\
\hline $\begin{array}{l}\text { Barium (total), in micrograms per } \\
\quad \text { liter }\end{array}$ & 2007 & 5 & 0 & 22.1 & 38.0 & 05/22/07 & 19.8 & 38.0 & -- & -- & -- & -- & -- & -- \\
\hline $\begin{array}{l}\text { Barium (total), in micrograms per } \\
\quad \text { liter }\end{array}$ & 2008-2009 & 12 & 0 & 22.3 & 30.3 & $06 / 17 / 08$ & 19.7 & 28.7 & -- & -- & -- & -- & -- & -- \\
\hline Cadmium, in micrograms per liter & $2001-2007$ & 34 & 32 & 4 & 0.07 & $10 / 11 / 01$ & 4 & 4 & -- & 0.23 & 0 & 0.85 & 0 & $\mathrm{~L}$ \\
\hline Cadmium, in micrograms per liter & 2008-2009 & 12 & 11 & 4 & ${ }^{5}<0.04$ & -- & 4 & 4 & -- & 0.23 & 0 & 0.85 & 0 & $\mathrm{~L}$ \\
\hline Chromium, in micrograms per liter & 2007 & 5 & 4 & 4 & ${ }^{5}<0.12$ & -- & 4 & 4 & -- & -- & -- & -- & -- & -- \\
\hline
\end{tabular}


Table 24. Summary of measured constituents and properties for Cebolla Creek at Powderhorn, Colorado, station 381633107054700.-Continued

[--, no data or not applicable; L, low; M, Medium; H, High; NTRU, Nephelometric Turbidity Ratio Unit; E. coli, Escherichia coli; mg/m² milligrams per square meter; USEPA, U.S. Environmental Protection Agency]

\begin{tabular}{|c|c|c|c|c|c|c|c|c|c|c|c|c|c|c|}
\hline $\begin{array}{l}\text { Property } \\
\text { or } \\
\text { constituent }\end{array}$ & $\begin{array}{l}\text { Period } \\
\text { (water } \\
\text { years) }\end{array}$ & $\begin{array}{c}\text { Number } \\
\text { of } \\
\text { samples }\end{array}$ & $\begin{array}{c}\text { Number } \\
\text { of } \\
\text { censored } \\
\text { values }\end{array}$ & Median $^{1}$ & Maximum & $\begin{array}{c}\text { Date } \\
\text { of } \\
\text { Maximum }\end{array}$ & $\begin{array}{c}\text { 15th } \\
\text { percentile }\end{array}$ & $\begin{array}{c}\text { 85th } \\
\text { percentile }\end{array}$ & $\begin{array}{c}\text { Geometric } \\
\text { Mean }^{2}\end{array}$ & $\begin{array}{c}\text { Standard } \\
\text { or } \\
\text { Chronic } \\
\text { standard }\end{array}$ & $\begin{array}{c}\text { Number of } \\
\text { exceedences } \\
\text { Standard or } \\
\text { Chronic }\end{array}$ & $\begin{array}{l}\text { Acute } \\
\text { standard }\end{array}$ & $\begin{array}{c}\text { Number } \\
\text { of } \\
\text { exceedences } \\
\text { Acute }\end{array}$ & $\begin{array}{c}\text { Level } \\
\text { of } \\
\text { concern }\end{array}$ \\
\hline Chromium, in micrograms per liter & $2008-2009$ & 12 & 10 & 4 & 0.17 & $10 / 28 / 08$ & 4 & 0.07 & -- & -- & -- & -- & -- & -- \\
\hline $\begin{array}{l}\text { Chromium (total), in micrograms } \\
\text { per liter }\end{array}$ & 2007 & 5 & 5 & 4 & ${ }^{5}<0.6$ & -- & 4 & 4 & -- & -- & -- & -- & -- & -- \\
\hline $\begin{array}{l}\text { Chromium (total), in micrograms } \\
\text { per liter }\end{array}$ & $2008-2009$ & 12 & 8 & 4 & ${ }^{5}<0.4$ & -- & 4 & 4 & -- & -- & -- & -- & -- & -- \\
\hline Copper, in micrograms per liter & $2001-2007$ & 34 & 2 & 0.61 & 1.2 & $02 / 06 / 07$ & 0.34 & 0.97 & -- & 4.6 & 0 & 6.4 & 0 & $\mathrm{~L}$ \\
\hline Copper, in micrograms per liter & $2008-2009$ & 12 & 9 & 4 & 0.90 & 05/28/09 & 4 & 0.83 & -- & 4.6 & 0 & 6.4 & 0 & $\mathrm{~L}$ \\
\hline Iron, in micrograms per liter & $2008-2009$ & 12 & 0 & 88 & 138 & 07/23/09 & 41 & 128 & -- & 300 & 0 & -- & -- & $\mathrm{L}$ \\
\hline Lead, in micrograms per liter & $2001-2007$ & 34 & 32 & 4 & ${ }^{s}<0.12$ & -- & 4 & 4 & -- & 1.05 & 0 & 27.0 & 0 & $\mathrm{~L}$ \\
\hline Lead, in micrograms per liter & $2008-2009$ & 12 & 8 & 4 & 0.07 & $06 / 17 / 08$ & 4 & 0.06 & -- & 1.05 & 0 & 27.0 & 0 & $\mathrm{~L}$ \\
\hline Manganese, in micrograms per liter & $2001-2007$ & 34 & 0 & 20.5 & 93.2 & $06 / 10 / 02$ & 11.5 & 44.6 & -- & 1,267 & 0 & 2,293 & 0 & $\mathrm{~L}$ \\
\hline Manganese, in micrograms per liter & $2008-2009$ & 12 & 0 & 17.7 & 34.1 & 05/28/09 & 13.2 & 33.5 & -- & 1,267 & 0 & 2,293 & 0 & $\mathrm{~L}$ \\
\hline Silver, in micrograms per liter & $2001-2007$ & 34 & 34 & 4 & ${ }^{5}<0.2$ & -- & 4 & 4 & -- & 0.02 & 0 & 0.52 & 0 & $\mathrm{~L}$ \\
\hline Silver, in micrograms per liter & $2008-2009$ & 12 & 12 & 4 & ${ }^{5}<0.1$ & -- & 4 & 4 & -- & 0.02 & 0 & 0.52 & 0 & $\mathrm{~L}$ \\
\hline Zinc, in micrograms per liter & $2001-2007$ & 34 & 22 & 4 & 7.0 & $08 / 22 / 07$ & 4 & 1.4 & -- & 63.3 & 0 & 73.0 & 0 & $\mathrm{~L}$ \\
\hline Zinc, in micrograms per liter & $2008-2009$ & 12 & 8 & 4 & 2.9 & 03/20/08 & 4 & 2.7 & -- & 63.3 & 0 & 73.0 & 0 & $\mathrm{~L}$ \\
\hline $\begin{array}{l}\text { Suspended sediment, in milligrams } \\
\text { per liter }\end{array}$ & $1999-2000$ & 4 & 0 & 12.9 & 93.3 & $06 / 01 / 99$ & 2.6 & 93.3 & -- & -- & -- & -- & -- & -- \\
\hline $\begin{array}{l}\text { Suspended sediment, in milligrams } \\
\text { per liter }\end{array}$ & $2008-2009$ & 12 & 0 & 4.5 & 38.0 & $02 / 22 / 08$ & 1.0 & 28.5 & -- & -- & -- & -- & -- & -- \\
\hline
\end{tabular}

per liter

Censored values were replaced with 0 to compute median and 85 th percentiles (coliform censored values replaced with 1) see definitions section.

${ }^{2}$ Geometric mean listed for $E$. coli. Geometric mean computed for combined period of record (2007-2009).

${ }^{3}$ Colorado Department of Public Health and Environment, classification and numeric standards for Gunnison and Lower Dolores River Basins (2010a); and USEPA National Recommended Water Quality Criteria (2009).

${ }^{4}$ Median values and percentiles were not tabled when they were calculated as zero.

${ }^{5}$ All values below laboratory reporting level (LRL), current LRL used for current period maximum, highest LRL used for historic period.

${ }^{6}$ Use geometric mean for comparison to standard. 
Table 25. Summary of seasonal Kendall trend tests for Cebolla Creek at Powderhorn, Colorado, station 381633107054700.

\begin{tabular}{|c|c|c|c|c|}
\hline Property or constituent & $\begin{array}{c}\text { Period } \\
\text { (water years) }\end{array}$ & Kendall tau $(\tau)$ & p-Value & Trend direction \\
\hline Specific conductance, in microsiemens per centimeter & $2002-2009$ & -0.205 & 0.253 & None \\
\hline $\mathrm{pH}$, in standard units & $2002-2009$ & -0.254 & 0.166 & None \\
\hline Ammonia plus organic nitrogen, in milligrams per liter & $2002-2009$ & -0.222 & 0.361 & None \\
\hline Phosphorus (total), in milligrams per liter & $2002-2009$ & -0.178 & 0.327 & None \\
\hline Orthophosphate, in milligrams per liter & $2002-2009$ & 0.000 & 1.000 & None \\
\hline Calcium, in milligrams per liter & $2002-2009$ & -0.370 & 0.034 & Down \\
\hline Magnesium, in milligrams per liter & $2002-2009$ & -0.507 & 0.003 & Down \\
\hline Manganese, in micrograms per liter & $2002-2009$ & -0.014 & 1.000 & None \\
\hline
\end{tabular}




\section{Mt. Crested Butte Wastewater Treatment Plant Effluent}

Current Reason for Inclusion: Assess phosphorus concentrations in treated wastewater treatment plant effluent.

\section{General Station Information:}

Station Type: $\quad$ USGS water quality

Period of Record: $\quad$ Water quality: Februrary 2009-September 2009

Latitude: $385330 \quad$ Drainage area: N/A HUC: 14020001

Longitude: $1065814 \quad$ Stream segment: N/A

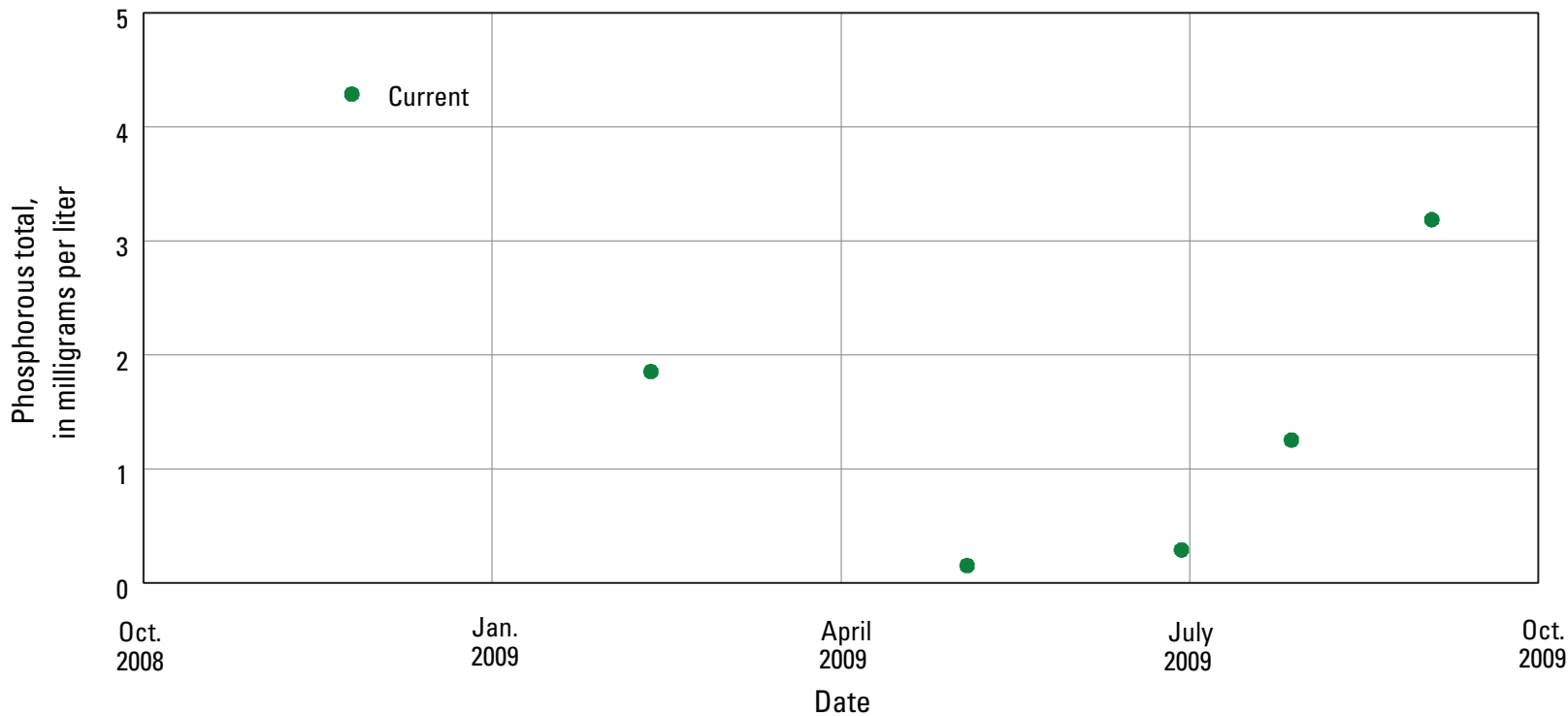

Figure 30. Time-series plots of selected water-quality constituents for Mt. Crested Butte Wastewater Treatment Plant. 
Table 26. Summary of measured constituents and properties for Mt. Crested Butte Wastewater Treatment Plant, station 385330106581401

\begin{tabular}{|c|c|c|c|c|c|c|c|c|}
\hline $\begin{array}{c}\text { Constituent } \\
\text { or } \\
\text { property }\end{array}$ & $\begin{array}{c}\text { Period } \\
\text { water years) }\end{array}$ & $\begin{array}{c}\text { Number } \\
\text { of } \\
\text { samples }\end{array}$ & $\begin{array}{c}\text { Number } \\
\text { of } \\
\text { censored } \\
\text { values }\end{array}$ & Median & Maximum & $\begin{array}{c}\text { Date } \\
\text { of } \\
\text { Maximum }\end{array}$ & $\begin{array}{c}\text { 15th } \\
\text { percentile }\end{array}$ & $\begin{array}{c}\text { 85th } \\
\text { percentile }\end{array}$ \\
\hline Phosphorus (total), in milligrams per liter & 2009 & 5 & 0 & 1.25 & 3.19 & $09 / 03 / 09$ & 0.151 & 3.19 \\
\hline
\end{tabular}




\section{Crested Butte Wastewater Treatment Plant Effluent}

Current Reason for Inclusion: Assess phosphorus concentrations in treated wastewater treatment plant effluent.

\section{General Station Information:}

Station Type:

USGS water quality

Period of Record:

Water quality: December 2001-September 2009

Latitude: 385229

Drainage area: N/A

HUC:14020001

Longitude: 106583

Stream segment: N/A

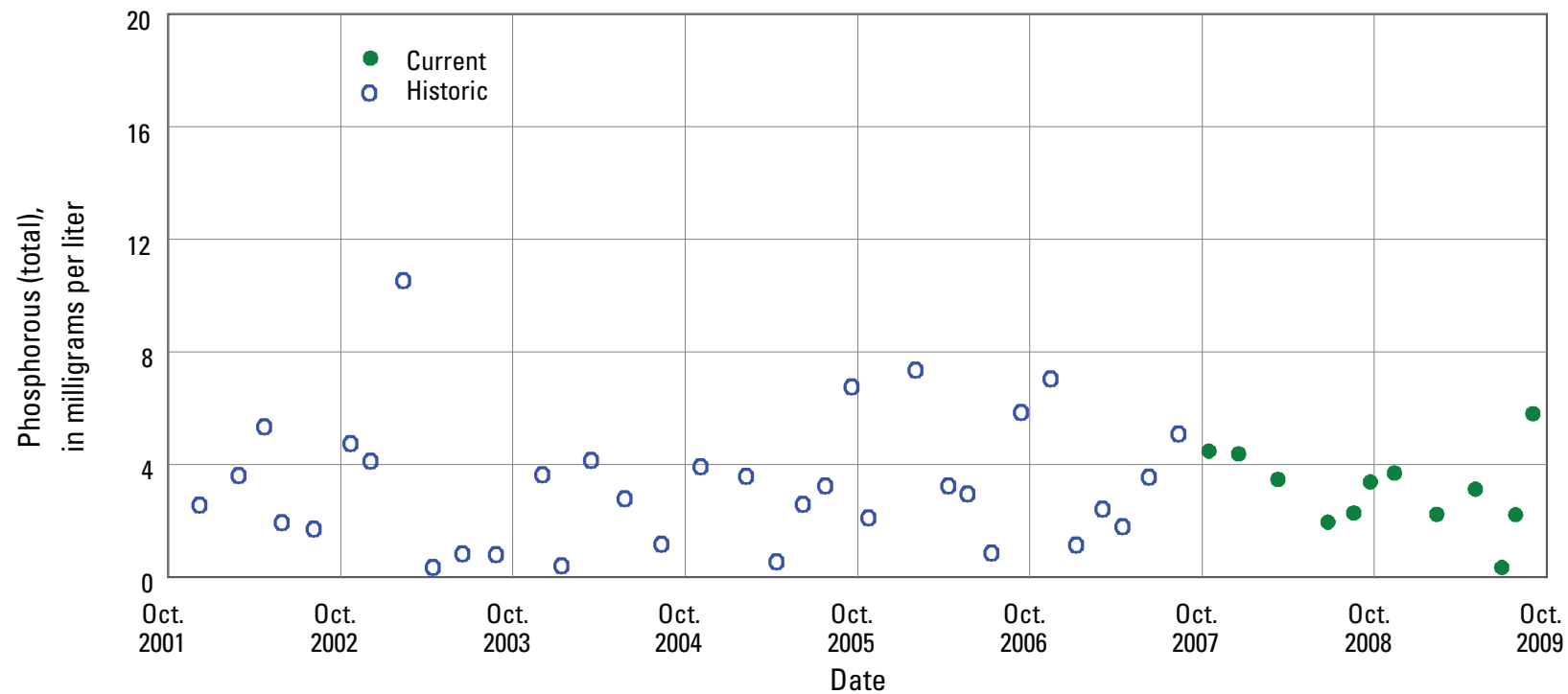

Figure 31. Time-series plots of selected water-quality constituents for Crested Butte Wastewater Treatment Plant. 
Table 27. Summary of measured constituents and properties for Crested Butte Wastewater Treatment Plant, station 385229106583100.

\begin{tabular}{|c|c|c|c|c|c|c|c|c|}
\hline $\begin{array}{c}\text { Constituent } \\
\text { or } \\
\text { property }\end{array}$ & $\begin{array}{l}\text { Period } \\
\text { (water } \\
\text { years) }\end{array}$ & $\begin{array}{c}\text { Number } \\
\text { of } \\
\text { samples }\end{array}$ & $\begin{array}{c}\text { Number } \\
\text { of } \\
\text { censored } \\
\text { values }\end{array}$ & Median & Maximum & $\begin{array}{c}\text { Date } \\
\text { of } \\
\text { Maximum }\end{array}$ & $\begin{array}{c}\text { 15th } \\
\text { percentile }\end{array}$ & $\begin{array}{c}\text { 85th } \\
\text { percentile }\end{array}$ \\
\hline Phosphorus (total), in milligrams per liter & $2002-2007$ & 34 & 0 & 3.10 & 10.6 & $02 / 11 / 03$ & 0.856 & 5.73 \\
\hline Phosphorus (total), in milligrams per liter & $2008-2009$ & 12 & 0 & 3.26 & 5.82 & $09 / 03 / 09$ & 1.88 & 4.56 \\
\hline
\end{tabular}

\section{References Cited}

Childress, C.J. Oblinger, Foreman, W.T., Connor, B.F., and Maloney, T.J., 1999, New reporting procedures based on long-term method detection levels and some considerations for interpretations of water-quality data provided by the U.S. Geological Survey National Water Quality Laboratory: U.S. Geological Survey Open-File Report 99-193, 19 p.

Colorado Department of Public Health and Environment, Water Quality Control Commission, 2010a, Classification and numeric standards for Gunnison and lower Dolores River Basins, Regulation no. 35: Denver, Colorado Department of Public Health and Environment, Water Quality Control Commission [variously paginated].Available at http://www.cdphe.state.co.us/regulations/wqccregs/.

Colorado Department of Public Health and Environment, Water Quality Control Commission, 2010b, The basic standards and methodologies for surface water (5CCR 1002-31), Regulation no. 31: Denver, Colorado Department of Public Health and Environment, Water Quality Control Commission [variously paginated]. Available at http://www.cdphe.state.co.us/regulations/wqccregs/.

Helsel, D.R., and Hirsch, R.M, 2002, Statistical methods in water resources: New York, Elsevier Science, 524 p.

U.S. Environmental Protection Agency, 2009, National recommended water quality criteria: U.S. Environmental Protection Agency, available at http://water.epa.gov/scitech/swguidance/standards/current/index.cfm.

U.S. Geological Survey, variously dated, National field manual for the collection of water-quality data: U.S. Geological Survey Techniques of Water-Resources Investigations, book 9, chaps. A1-A9, available online at http://pubs.water.usgs.gov/twri9A. 
